\title{
Wasserstoffabsorption in epitaktischen Niobschichten: eine STM-Studie
}

\author{
Dissertation \\ zur Erlangung des Doktorgrades \\ der Mathematisch-Naturwissenschaftlichen Fakultäten \\ der Georg-August-Universität zu Göttingen
}

vorgelegt von

Kai Nörthemann

aus

Duderstadt

Göttingen 2006 
D7

Referentin:

PD. Dr. Astrid Pundt

Korreferent:

Prof. Dr. Hans Hofsäß

Tag der Disputation:

11.12.2006 


\section{Inhaltsverzeichnis}

1 Einleitung $\quad 1$

2 Wasserstoff in Metallen 3

2.1 Das massive Niob-Wasserstoffsystem . . . . . . . . . . . . . . 3

2.1 .1 Phasendiagramm . . . . . . . . . . . . . . 3

2.1.2 Gitterdehnung . . . . . . . . . . . . . . . 5

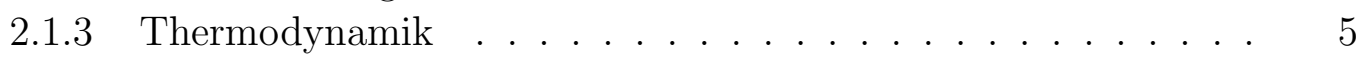

2.2 Dünne Niob-Wasserstoff-Schichten . . . . . . . . . . . . . . . . 6

2.2.1 Phasendiagramm . . . . . . . . . . . . . . . . . . 7

2.2 .2 Gitterdehnung . . . . . . . . . . . . . . . . . 7

2.2.3 Thermodynamik ................... 8

2.2.4 Mechanische Spannungen in haftenden Schichten . . . . . . . 9

2.3 Diffusion in dünnen Schichten . . . . . . . . . . . . . . . . . 14

2.4 Wasserstoffbeladung aus der Gasphase . . . . . . . . . . . . . 16

3 Experimentelle Methoden $\quad 17$

3.1 Eingesetzte Apparaturen . . . . . . . . . . . . . . . . . . . . . . 17

3.1 .1 UHV-Anlage . . . . . . . . . . . . . . . . . . . . . . . . . 17

3.1 .2 Gasbeladung . . . . . . . . . . . . . . . . . . 19

3.1.3 Rastertunnelmikroskopie . . . . . . . . . . . . . . . 20

3.2 Herstellung und Charakterisierung von Nb-Schichten . . . . . . . . . 23

3.2.1 Präparation epitaktischer Niobschichten . . . . . . . . . . . 24

3.2.2 Charakterisierung der Schichten . . . . . . . . . . . . 25

3.2.3 Niobschichten für die Gasbeladung . . . . . . . . . . . . . . . 29

3.2.4 Auftreten mechanischer Spannung in STM-Messungen . . . . 30

3.3 Finite-Elemente-Methode . . . . . . . . . . . . . . . . 35

3.3.1 Ausscheidung in einer dünnen Schicht . . . . . . . . . . . . . 37

3.3.2 Ausscheidung in einer Drahtgeometrie . . . . . . . . . . . 42

4 Experimentelle Resultate 45

4.1 Wasserstoffaufnahme in der $\alpha$-Phase . . . . . . . . . . . . . . 45

4.1.1 Kinetik bei der Beladung . . . . . . . . . . . . . . . . . 45

4.1.2 Kinetik bei der Entladung . . . . . . . . . . . . . . . 47

4.1.3 Maximaldruck der $\alpha$-Phase bei Raumtemperatur . . . . . . . . 47

4.1.4 Oberflächenmorphologie innerhalb der $\alpha$-Phase . . . . . . . . 49 
4.2 Hydridbildung in epitaktischen Schichten . . . . . . . . . . . . . 51

4.2.1 Schichtdickenabhängigkeit der vertikalen lokalen Ausdehnung des Hydrids im Zweiphasengebiet . . . . . . . . . . . . . 51

4.2.2 Unterschiedliche Ausscheidungstypen . . . . . . . . . . . 56

4.2.3 Einflussparameter auf die Hydridbildung . . . . . . . . . . . . 59

4.2.4 Oberflächenmorphologie des Hydrids . . . . . . . . . . . . . 60

4.2.5 Präferentielle Wachstumsrichtung des Hydrids . . . . . . . . . 61

4.2.6 Versetzungen im Hydrid und Bestimmung des Gleitsystems . . 64

4.2.7 Randbereich zwischen $\alpha$-Phase und Hydrid . . . . . . . . . . . 66

4.3 Keimbildung und Wachstum . . . . . . . . . . . . . . . . 69

4.3.1 Anfangsstadien . . . . . . . . . . . . . . . . . 69

4.3.2 Umwandlung der Ausscheidungstypen . . . . . . . . . . . . . . 72

4.3.3 Zeitliche Entwicklung der Hydridausscheidungen bis $3 \mathrm{~h}$. . . . 75

4.3.4 Zeitliche Entwicklung der Ausscheidungen bis $170 \mathrm{~h}$. . . . . . 78

4.4 Laterale Schichtausdehnung . . . . . . . . . . . . . . . 80

5 Diskussion 83

5.1 Wasserstoffaufnahme in der $\alpha$-Phase und Randlöslichkeit . . . . . . . 83

5.2 Hydridbildung . . . . . . . . . . . . . . . . . . . . . . 84

5.2.1 Ausscheidungen vom Typ $\mathrm{T} 1 \ldots \ldots$. . . . . . . . . 84

5.2 .2 Ausscheidungen vom Typ $\mathrm{T} 2 \ldots \ldots$. . . . . . . . . . . . 89

5.2.3 Randbereich zwischen $\alpha$-Phase und T2 Ausscheidungen . . . . 90

5.2.4 Einflussparameter auf die unterschiedlichen Ausscheidungstypen 91

5.3 Keimbildung und Wachstum . . . . . . . . . . . . . . . . 93

5.3.1 Anfangsstadien . . . . . . . . . . . . . . . . . 94

5.3 .2 Sättigung . . . . . . . . . . . . . . . . . . 94

$\begin{array}{lll}6 & \text { Zusammenfassung } & 97\end{array}$

$\begin{array}{ll}\text { Literaturverzeichnis } & 101\end{array}$

$\begin{array}{ll}\text { Worte des Dankes } & 107\end{array}$

$\begin{array}{ll}\text { Lebenslauf } & 109\end{array}$ 


\section{Abbildungsverzeichnis}

1.1 Hydridbildung in einer dünnen Schicht . . . . . . . . . . . . . . . . 2

2.1 Oktaeder $(\mathrm{O})$ und Tetraeder (T) Plätze im bcc-Niob-Wirtsgitter . . . 3

2.2 Phasendiagramm des massiven Niob-Wasserstoff-Systems [SW78] . . . 4

2.3 Volumenänderung von massivem Niob in Abhängigkeit von der Wasserstoffkonzentration $[\mathrm{SW} 78] \ldots \ldots \ldots \ldots$

2.4 Isotherme für bulk Niob-Wasserstoff $[\mathrm{PT} 69] \ldots \ldots$. . . . . . . . . . 6

2.5 Plateaudruck der Hydridbildung für massives Niob [PT69] . . . . . . 7

2.6 Modell zur Beschreibung der Dehnung in einem mit Wasserstoff beladenen dünnen Film . . . . . . . . . . . . . . . . . . . . . 8

2.7 EMK einer $100 \mathrm{~nm}$ dünnen Nb-Schicht in Abhängigkeit von der Wasserstoffkonzentration . . . . . . . . . . . . . . 99 9

2.8 Richtungsabhängiger Elastizitätsmodul von Niob . . . . . . . . . . 12

2.9 Einfluss von Versetzungen auf die Oberflächenmorphologie einer dünnen Schicht . . . . . . . . . . . . . . . . . . . . . . . 14

2.10 Randbedingungen für die Lösung der Diffusionsgleichung für dünne Schichten . . . . . . . . . . . . . . . . . 14

3.1 Schematische Darstellung der eingesetzten UHV-Kammer . . . . . . . 18

3.2 Experimenteller Aufbau der Gasbeladung und Druckverlauf während einer Wasserstoffbeladung . . . . . . . . . . . . . . . . . 19

3.3 Grundprinzipien topographischer Rastertunnelmikroskopie . . . . . . 20

3.4 MicroSTM der Firma Omicron innerhalb der UHV-Kammer . . . . . 22

3.5 Schematischer Aufbau der eingesetzten Zweigitter Ar-Ionenquelle . . 23

3.6 Restgasanalyse während der Schichtpräparation . . . . . . . . . . 24

3.7 Orientierungsbeziehung von Niob auf einem Saphirsubstrat . . . . . . 25

3.8 Texturmessung eines $100 \mathrm{~nm}$ dünnen Niobfilms . . . . . . . . . . . 26

3.9 STM-Aufnahme eines $100 \mathrm{~nm}$ dünnen Niobfilms direkt nach der Schichtherstellung . . . . . . . . . . . . . . . . . . 27

3.10 STM-Aufnahme eines $20 \mathrm{~nm}$ dünnen Niobfilms . . . . . . . . . . 28

3.11 Höhenprofile von Schichten mit einer Dicke von $20 \mathrm{~nm}$ und $100 \mathrm{~nm}$. . 28

$3.12100 \mathrm{~nm}$ dünner Niobfilm, geringfügig mit Palladium bedeckt . . . . . 29

3.13 Variation des Wasserstoffdrucks bei einer STM-Messung eines $100 \mathrm{~nm}$ dünnen Niobfilms . . . . . . . . . . . . . . . . . . . 30

3.14 Höhenprofile der STM-Aufnahme von Abb. 3.13 . . . . . . . . . 31 
3.15 Beladung einer $50 \mathrm{~nm}$ dünnen Niobschicht bei einem Wasserstoffdruck von $1.6 \cdot 10^{-7}$ mbar . . . . . . . . . . . . . . . . . . . . . . .

3.16 Beladung einer $50 \mathrm{~nm}$ dünnen Niobschicht bei einem Wasserstoffdruck von $1.4 \cdot 10^{-6}$ mbar . . . . . . . . . . . . . . . . 33

3.17 Krümmung des Substrats durch Spannungen im beladenen Niobfilm . 34

3.18 Berechnetes Gebiet der Finite-Elemente-Methode für die Schichtgeometrie . . . . . . . . . . . . . . . . .

3.19 Totale Verschiebung einer zylinderförmigen Ausscheidung, berechnet mit der Finite-Elemente-Methode . . . . . . . . . . . . . . . . . .

3.20 Vergleich der an der Oberfläche sichtbaren Höhenveränderung bei unterschiedlichen Positionen und Ausscheidungsformen . . . . . . . . . .

3.21 An der Oberfläche sichtbare Höhenveränderung bei unterschiedlichen Ausscheidungsformen . . . . . . . . . . . . . . .

3.22 Elastische Energie, die in zylinderförmigen Ausscheidungen gespeichert ist . . . . . . . . . . . . . . . . . .

3.23 Berechnetes Gebiet bei der Finite-Elemente-Methode für die Drahtgeometrie . . . . . . . . . . . . . . . .

3.24 Dehnung eines Niobdrahts mit einer quaderförmigen Ausscheidung.

3.25 Höhenprofile der Ausdehnung bei einer Drahtgeometrie . . . . . . . .

4.1 Desorbtion von Wasserstoff bei Druckerniedrigung . . . . . . . . . . .

4.2 Wasserstoffdruck der Hydridbildung für Niobfilme mit unterschiedlicher Schichtdicke

4.3 Differenzbild von STM-Messungen vor und nach Hydridentstehung bei einem $50 \mathrm{~nm}$ dünnen Niobfilm . . . . . . . . . . . . . . 50

4.4 Hydridbildung in dünnen Schichten . . . . . . . . . . . . . . . 51

$4.572 \mathrm{~nm}$ dünner Niobfilm, beladen bei $5 \cdot 10^{-6}$ mbar Wasserstoffdruck . . 52

$4.680 \mathrm{~nm}$ dünner Niobfilm, beladen bei $6 \cdot 10^{-7}$ mbar Wasserstoffdruck . . 53

4.7 Koexistenz von Hydrid und $\alpha$-Phase bei einem $100 \mathrm{~nm}$ Niobfilm . . . 53

$4.8120 \mathrm{~nm}$ dünner Niobfilm, beladen bei $8.2 \cdot 10^{-7}$ mbar Wasserstoffdruck 54

4.9 Schematische Verteilung des Hydrids in der Schicht . . . . . . . . . 56

4.10 Höhenprofile entlang des Übergangs von der $\alpha$-Phase zum Hydrid eines $80 \mathrm{~nm}$ dünnen Niobfilms aus Abb. $4.6 \ldots$. . . . . . . . . 57

4.11 Unterschiedliche Ausscheidungstypen eines $70 \mathrm{~nm}$ dünnen Niobfilms . 58

4.12 Veränderung der Oberflächenunebenheit bei der Hydridbildung in einem $100 \mathrm{~nm}$ dünnen Niobfilm . . . . . . . . . . . . . . . . 60

4.13 Orientierung der Ausscheidungen vom Typ T1 . . . . . . . . . . . 62

4.14 Bestimmung der Filmorientierung eines $100 \mathrm{~nm}$ dünnen Niobfilms . . 63

4.15 Bestimmung der Vorzugsrichtung der Hydridbildung . . . . . . . . . . 63

4.16 Gleitstufen in einer $100 \mathrm{~nm}$ dünnen Niobschicht, sichtbar in den Gebieten in denen sich Hydrid gebildet hat . . . . . . . . . . . . . .

4.17 Sichtbare Gleitstufen in Gebieten der $\alpha$-Phase und des Hydrids bei

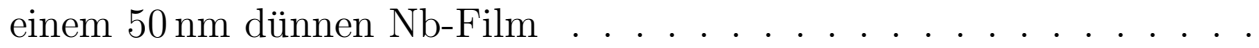


4.18 Höhenprofile der Gleitstufen aus Abb. 4.17 eines $50 \mathrm{~nm}$ Niobfilms . .

4.19 Winkelverteilung der Gleitstufen eines $50 \mathrm{~nm}$ dünnen Niobfilms aus Abb. $4.17 \ldots \ldots \ldots \ldots \ldots$

4.20 Zeitliche Entwicklung des Hydridwachstums einer $70 \mathrm{~nm}$ dünnen Niobschicht . . . . . . . . . . . . . . . .

4.21 Höhenverteilungen der STM-Bilder einer $70 \mathrm{~nm}$ dünnen Niobschicht

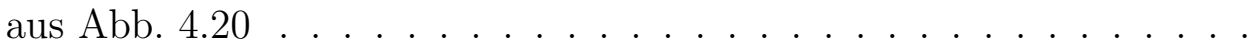

4.22 Zeitliche Entwicklung des Hydrids eines $70 \mathrm{~nm}$ dünnen Niobfilms aus Abb. $4.20 \ldots \ldots \ldots \ldots \ldots$

4.23 Maximalhöhe der Ausscheidungen in Abhängigkeit von ihrer Fläche .

4.24 Häufigkeitsverteilung der Größe der Ausscheidungen der STM-Bilder aus Abb. $4.20 \ldots \ldots \ldots \ldots$. . . . . . . . . . . . . 74

4.25 Umwandlung der Ausscheidungen vom Typ T1 zum Typ T2 . . . . .

4.26 STM-Bilder eines $30 \mathrm{~nm}$ dünnen Niobfilms bei unterschiedlichen Wasserstoffdrücken . . . . . . . . . . . . . . 76

4.27 Hydridwachstum des $30 \mathrm{~nm}$ dünnen Niobfilms von Abb. 4.26 . . . . . 77

4.28 STM-Bilder der zeitlichen Entwicklung der Hydridbildung eines $80 \mathrm{~nm}$ dünnen Niobfilms . . . . . . . . . . . . . . . . . . .

4.29 Ausscheidungswachstum einer $80 \mathrm{~nm}$ dünnen Niobschicht bei einem Wasserstoffdruck von $9 \cdot 10^{-7}$ mbar . . . . . . . . . . . . . . .

4.30 Veränderungen einer $20 \mathrm{~nm}$ dünnen Niobschicht während der Wasserstoffbeladung ........................ . . 81

4.31 Höhenprofil des Differenzbildes aus Abb. 4.30(e) . . . . . . . . . . 82

5.1 Querschnitt durch Ausscheidung und Matrix mit unterschiedlichen Gitterkonstanten . . . . . . . . . . . . . . . . .

5.2 Energiewert, bei dem in zylinderförmigen Ausscheidungen Versetzungen gebildet werden . . . . . . . . . . . . . . . . .

5.3 Vergleich von Höhenprofilen, berechnet mit der Finite-ElementeMethode und einer Messung einer $20 \mathrm{~nm}$ dünnen Niobschicht . . . . . 88

5.4 Schematischer Querschnitt von Typ T1-Ausscheidungen . . . . . . . . 88

5.5 Schematischer Querschnitt einer Typ T2-Ausscheidung . . . . . . . . 89

5.6 Bevorzugtes Gleitsystem, eingezeichnet in die Einheitszelle . . . . . . 90

5.7 Entstehung von Gleitstufen im Randbereich der $\alpha$-Phase . . . . . . . 91

5.8 Unterschiedliche Ausscheidungstypen bei der Hydridbildung für verschiedene Schichteigenschaften . . . . . . . . . . . . . . . . . 92

5.9 Schematische Darstellung der Veränderung einer Isotherme beim Übergang zu dünnen Schichten . . . . . . . . . . . . . . . . . . 96 


\section{Tabellenverzeichnis}

3.1 Berechnung der induzierten Spannung aus der Substratkrümmung . .

4.1 Zeitabhängigkeit der Wasserstoffaufnahme bei verschiedenen Drücken und Schichtdicken . . . . . . . . . . . . . . . . 46

4.2 Höhenveränderungen zwischen Gebieten der $\alpha$-Phase und des Hydrids vom Typ T2 bei unterschiedlichen Schichtdicken . . . . . . . . . . . 55

4.3 Größe der Ausscheidungen vom Typ T1 . . . . . . . . . . . . . . . . 57

4.4 Zusammenstellung der Ausscheidungstypen für unterschiedliche Schichtdicken und Substratfehlschnitte . . . . . . . . . . . 59

4.5 Winkel zwischen den Schnittlinien unterschiedlicher Gleitebenen an der Oberfläche im bcc-Gitter . . . . . . . . . . . . . . . . . . .

5.1 Vergleich der lateralen Maximalgröße von Typ T1-Ausscheidungen mit der Ausscheidungsgröße, ab der sich Versetzungen bilden können . . . 


\section{Einleitung}

Nachdem T. Graham 1866 entdeckt hatte, dass sich Wasserstoff in Palladium löst [Gra66], wurde das Gebiet „Wasserstoff in Metallen“ Gegenstand intensiver Forschung. Der Einfluss von gelöstem Wasserstoff auf das betrachtete System ist von großer Bedeutung. Aufgrund der schnellen Diffusion des Wasserstoffs in Metallen sind Veränderungen bei Raumtemperatur schon nach kurzer Zeit beobachtbar, bei der andere Phänomene, wie z. B. Erholung oder Rekristallisation noch nicht in Erscheinung treten. Die Aufnahme von Wasserstoff in Metalle kann sowohl positive als auch negative Folgen haben. Als Beispiel für den negativen Einfluss der Wasserstoffaufnahme ist die Versprödung von Stahl zu nennen [Ori78]. Des Weiteren treten Veränderungen in den elektrischen, magnetischen und mechanischen Eigenschaften auf [AV78a]. Es ist z.B. mit Hilfe von Wasserstoff möglich, einen Metall-Halbleiterübergang in Yttrium reversibel zu schalten $\left[\mathrm{KvdMK}^{+} 00\right]$. Die Anwendung von Wasserstoff als Energieträger der Zukunft rückte in den letzten Jahren immer mehr in den Vordergrund [SZ01]. So haben die Nickelmetallhydrid-Akkumulatoren (NiMH) die Serienreife erreicht und werden mittlerweile von vielen Herstellern als Batterien angeboten.

In den Anwendungen werden in den letzten Jahren die Strukturen immer weiter verkleinert, so dass mesoskopische und nanoskalige Systeme zunehmend genutzt werden.

Durch die Verringerung der Dimension verändern sich die physikalischen Eigenschaften der verwendeten Materialien. In der Literatur wird u.a. beschrieben, dass die Phasengrenzen in Systemen mit reduzierten Dimensionen gegenüber den massiven Proben verschoben sind. H. ZABEL et.al. [SGAZ96, $\mathrm{ASS}^{+}$97, SRTBZ97] haben Messungen der Gitterkonstante von Niobschichten in Abhängigkeit von Wasserstoffdruck bei hohen Temperaturen für unterschiedliche Schichtdicken durchgeführt. Daraus folgern sie eine schichtdickenabhängige Abnahme der kritischen Temperatur $T_{c}$ der $\alpha$ - $\alpha^{\prime}$-Entmischung im Niob-Wasserstoff-System. M. DoRnHEIM zeigt, dass sich die Phasengrenzen bei Raumtemperatur im Fall von dünnen Metallwasserstoffschichten gegenüber den Werten für massive Proben verschieben [Dor02]. Viele Veränderungen der Eigenschaften in Proben mit reduzierter Dimension gegenüber den massiven Proben beruhen auf dem größeren Einfluss der Oberfläche, der Morphologie und der Mikrostruktur sowie den mechanischen Spannungen, die in auf Substraten haftenden Schichten bei der Wasserstoffbeladung entstehen [PK06].

In diesen Untersuchungen wurden unterschiedliche Metall-Wasserstoffsysteme bzw. die Hydridbildung makroskopisch gemittelt betrachtet und dabei beobachtet, dass in dünnen Schichten bei der Wasserstoffbeladung in Schichtebene große mechanische Spannungen entstehen $\left[\mathrm{LPB}^{+} 99\right]$, die zu einer starken vertikalen (out-of-plane) Deh- 
nung führen $\left[\mathrm{YSF}^{+} 96, \mathrm{LFK}^{+}\right.$99]. Bei der schrittweisen Wasserstoffbeladung wurde die Koexistenz von $\alpha$-Phase und Hydrid beobachtet.

Während die bisherigen Arbeiten mit makroskopischen Methoden gearbeitet haben, sollen hier die Veränderungen auf der Skala weniger Nanometer analysiert werden. Der Effekt der starken vertikalen Dehnung in dünnen Schichten wird dazu genutzt, um die Wasserstoffaufnahme im Zweiphasengebiet zu untersuchen und die Keimbildung und das Wachstum des Hydrids zu analysieren.

Eine genaue Analyse erfolgt damit mit Hilfe der Rastertunnelmikroskopie (STM), womit die lokale Veränderung der Probenoberfläche während der Wasserstoffbeladung gemessen werden kann. Bei dem beobachteten Ausschnitt liegt die laterale Auflösung im Bereich weniger Nanometer. Die vertikale Auflösung ist bei dieser Methode atomar und ermöglicht daher, geringe Unterschiede an der Filmoberfläche aufzulösen (vgl. Abb. 1.1).

Bei der Messung mit dem oberflächensensitiven Rastertunnelmikroskop

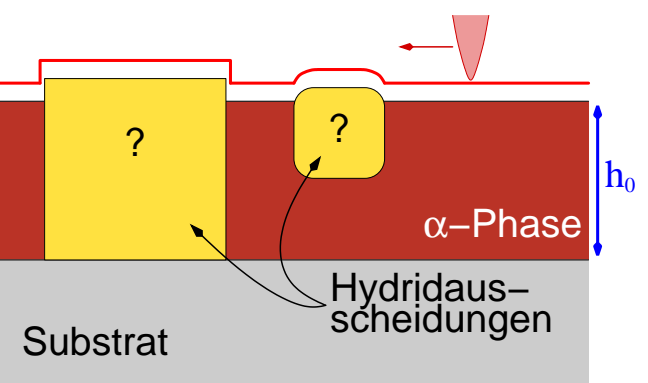

Abbildung 1.1: Hydridbildung in einer dünnen Schicht: An der Oberfläche sind mit dem STM Veränderungen sichtbar. stellt sich die Frage, inwieweit es möglich ist, aus gewonnenen Oberflächendaten die Veränderungen im Volumen zu bestimmen. Damit die Veränderungen im Volumen beschrieben werden können, wird das theoretische Modell der Elastizitätstheorie herangezogen, so dass aus den Höhenprofilen und bekannten Schichteigenschaften auf die Form der Hydridausscheidungen geschlossen werden kann.

Durch kontinuierliche Messung an einer Probenposition ist es möglich, die zeitliche Entwicklung der Hydridausscheidungen zu messen, um damit eine fortlaufende lokale Abbildung von Keimbildung und Wachstum zu erreichen. Die Phasenumwandlung wird durch eine Beladung aus der Gasphase herbeigeführt. Während der Beladung können Messungen durchgeführt und Veränderungen der Oberflächenmorphologie durch die Gassorption in dünnen Schichten mit einer hohen Auflösung gemessen werden.

Diese Arbeit gliedert sich wie folgt: Zunächst werden in Kapitel 2 die Grundlagen der Wechselwirkungen zwischen Wasserstoff und Niob behandelt. Dabei wird auf das Verhalten von massivem Niob eingegangen und die auftretenden Veränderungen für den Übergang zu dünnen Schichten behandelt. Die verwendeten experimentellen Methoden werden in Kapitel 3 beschrieben. Dabei wird auf die Präparation und Charakterisierung der Proben eingegangen, Effekte erörtert, die bei der Messung auftreten, und Berechnungen mit der Finite-Elemente-Methode zu Ausscheidungsgeometrien behandelt. In Kapitel 4 werden die Messungen der in-situ Gasbeladung von unterschiedlich dünnen epitaktischen Niobschichten beschrieben und ausgewertet und anschließend Ergebnisse in Kapitel 5 im Gesamtbild diskutiert. Den Abschluss bildet Kapitel 6 mit einer Zusammenfassung. 


\section{Wasserstoff in Metallen}

Als Ausgangspunkt für die Untersuchungen dünner epitaktischer Niobschichten sollen in diesem Kapitel zunächst in Abschnitt 2.1 die Grundlagen vom massiven Niob-Wasserstoffsystem beschrieben werden. Anschließend werden in Abschnitt 2.2 Veränderungen erläutert, die beim Übergang zu dünnen Schichten auftreten. In Abschnitt 2.3 wird die Diffusion in dünnen Schichten behandelt. Auf die theoretischen Grundlagen der Wasserstoffbeladung aus der Gasphase wird in Abschnitt 2.4 eingegangen.

\subsection{Das massive Niob-Wasserstoffsystem}

Wasserstoff wird interstitiell bevorzugt auf den Tetraederplätzen im kubisch raumzentrierten (bcc) Niobgitter gelöst. Die Oktaederplätze werden erst bei einer Konzentration $c>1 \mathrm{H} / \mathrm{Nb}$ besetzt. Diese Positionen sind in Abb. 2.1 dargestellt. Durch das Lösen der Wasserstoffatome auf den Zwischengitterplätzen wird das Niobgitter gedehnt (s. Abschnitt 2.1.2). Die Diffusion des Wasserstoffs ist aufgrund der interstitiellen Positionen und seiner geringen Atomgröße sehr schnell (s. Abschnitt 2.3).

\subsubsection{Phasendiagramm}

Das Phasendiagramm des Systems Niob-Wasserstoff ist in Abb. 2.2 dargestellt [AV78b]. Bei Zimmertemperatur treten drei unterschiedliche Phasen auf. Dies ist die

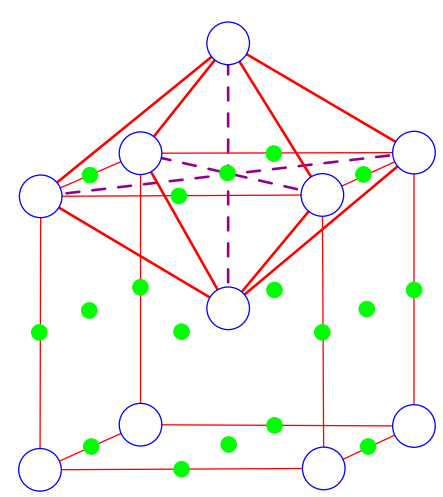

(O)

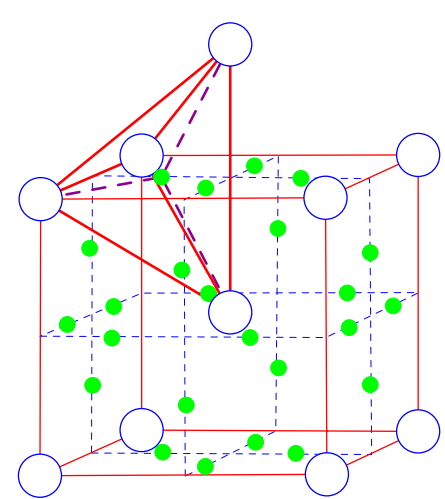

(T)

Abbildung 2.1: Oktaeder (O) und Tetraeder (T) Plätze im bcc-Niob-Wirtsgitter 


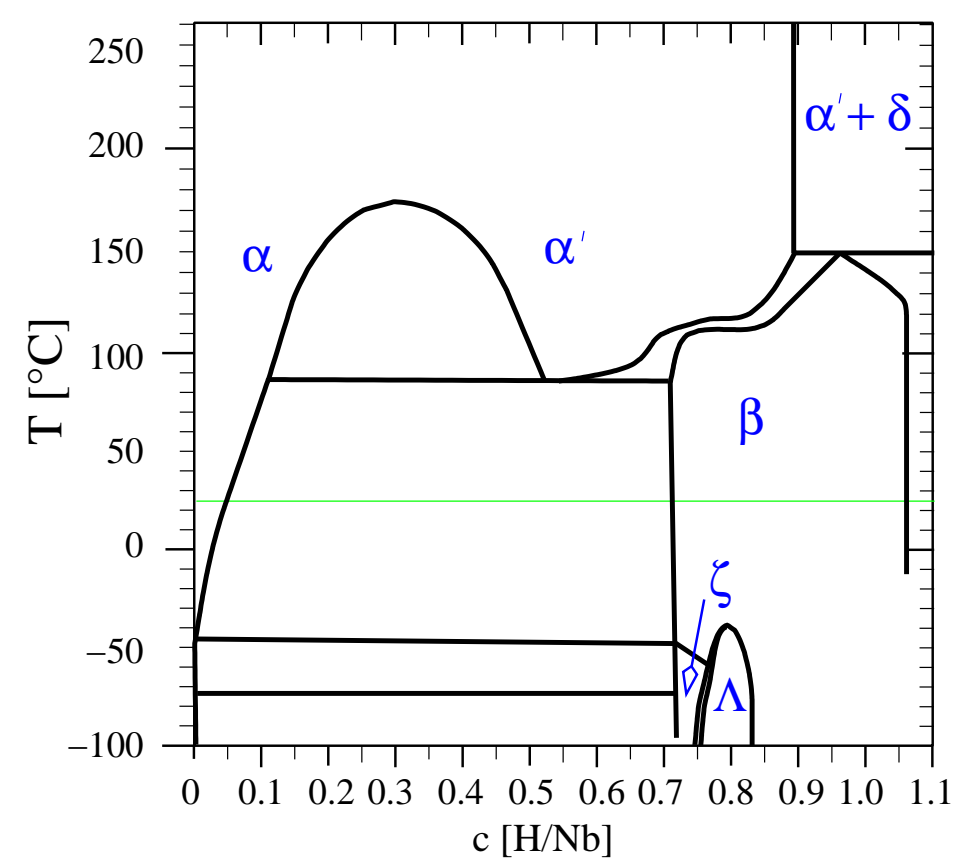

Abbildung 2.2: Phasendiagramm für massives Niob-Wasserstoff [SW78]. Bei Zimmertemperatur existieren drei Phasen: die $\alpha-, \beta$ - und $\delta$-Phase. Im Temperaturbereich von ca. $90{ }^{\circ} \mathrm{C}$ bis $180{ }^{\circ} \mathrm{C}$ tritt eine Entmischung zwischen der $\alpha$ - und $\alpha^{\prime}$-Phase auf.

$\alpha$-Phase, die wie reines Niob eine bcc-Gitterstruktur besitzt und bis zu einer Wasserstoffkonzentration von $0.06 \mathrm{H} / \mathrm{Nb}$ stabil ist. Der Wasserstoff ist hier ungeordnet verteilt. Bei höheren Konzentrationen wird zusätzlich die $\beta$-Phase (Hydrid) gebildet, die gegenüber der $\alpha$-Phase gedehnt ist und eine orthorombische Gitterstruktur besitzt. Die Verzerrung der rechtwinkligen Einheitszelle mit einer quadratischen Grundfläche beträgt nur $c / a=1.005$ [WR70]. Die $(\alpha+\beta)$ Mischungslücke erstreckt sich von $0.06 \mathrm{H} / \mathrm{Nb}$ bis $0.72 \mathrm{H} / \mathrm{Nb}$ und hat somit eine Breite von $0.66 \mathrm{H} / \mathrm{Nb}$. Ab einer Konzentration von $\approx 1.1 \mathrm{H} / \mathrm{Nb}$ wird die $\delta$-Phase ausgeschieden [WR70]. Ab einer Temperatur von ca. $90{ }^{\circ} \mathrm{C}$ wird zusätzlich noch die $\alpha^{\prime}$-Phase beobachtet. Diese unterscheidet sich von der $\alpha$-Phase bei gleicher Gitterstruktur durch eine höhere Konzentration. Oberhalb von ca. $180{ }^{\circ} \mathrm{C}$ können diese beiden Phasen nicht mehr unterschieden werden. Wie in Abb. 2.2 zu sehen ist, treten bei Temperaturen unterhalb der Zimmertemperatur noch weitere Phasen auf, die hier nicht weiter beschrieben werden, weil sich die Messungen auf Zimmertemperatur beschränken.

In dünnen Schichten wird aufgrund der Wechselwirkung zwischen Schicht und Substrat das Gitter der Schicht verzerrt. Folglich ist es schwer, zwischen einer verzerrten $\alpha^{\prime}$-Phase und einer orthorombischen $\beta$-Phase zu unterscheiden. Wegen dieser Problematik wird im Weiteren nicht zwischen beiden Phasen unterschieden, sondern von der Hydridphase gesprochen. 


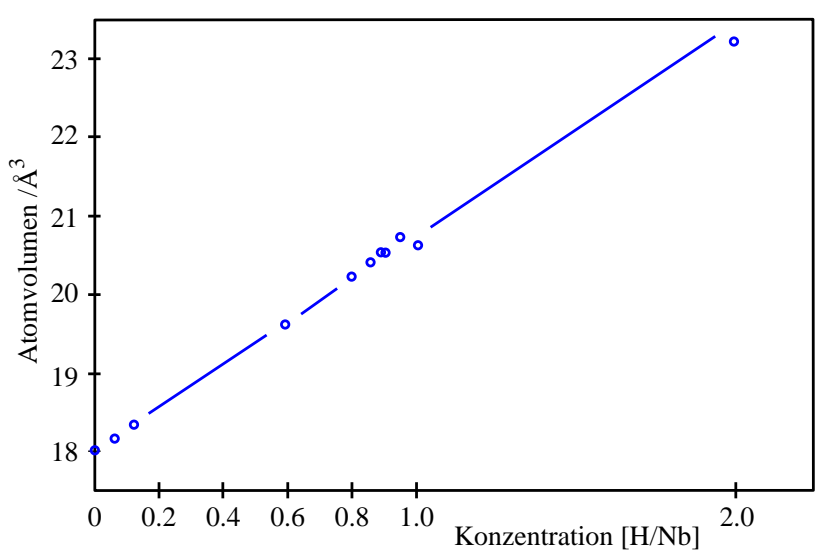

Abbildung 2.3: Volumenänderung von massivem Niob in Abhängigkeit von der Wasserstoffkonzentration [SW78]. Das Atomvolumen steigt über einen großen Bereich linear mit der Wasserstoffkonzentration.

\subsubsection{Gitterdehnung}

In Abb. 2.3 ist dargestellt, dass das Volumen des Niob-Gitters linear mit der Wasserstoffkonzentration ansteigt. H. PEISL gibt eine Expansion im Bereich der $\alpha$-Phase für Einkristalle bei Zimmertemperatur von

$$
\frac{\Delta V}{V_{0}}=\frac{\Delta v}{\Omega} \cdot c_{\mathrm{H}}=0.174(5) \cdot c_{\mathrm{H}}
$$

an [Pei78]. Von G. Brauer und R. Hermann wurde für Niobpulver ein Faktor von

$$
\frac{\Delta V}{V_{0}}=\frac{\Delta v}{\Omega} \cdot c_{\mathrm{H}}=0.16 \cdot c_{\mathrm{H}}
$$

im Bereich der $\beta$-Phase bei Zimmertemperatur $\left(T=20^{\circ} \mathrm{C}\right)$ gemessen [BH53]. Für kubische Kristalle mit zufälliger Verteilung der Zwischengitteratome, wie es in der $\alpha$-Phase der Fall ist, kann die Änderung im Volumen $\Delta V / V_{0}$ durch die Veränderung einer Länge (z.B. der Gitterkonstante) angegeben werden:

$$
\frac{\Delta V}{V_{0}}=3 \cdot \frac{\Delta a}{a_{0}}
$$

Damit ergibt sich über einen weiten Konzentrationsbereich der in Abb. 2.3 dargestellte lineare Zusammenhang.

\subsubsection{Thermodynamik}

In Abbildung 2.4 sind bei unterschiedlicher Temperatur aufgenommene Isothermen des Niob-Wasserstoff Systems aufgetragen (nach J. A. Pryde und C. G. Titcomb 


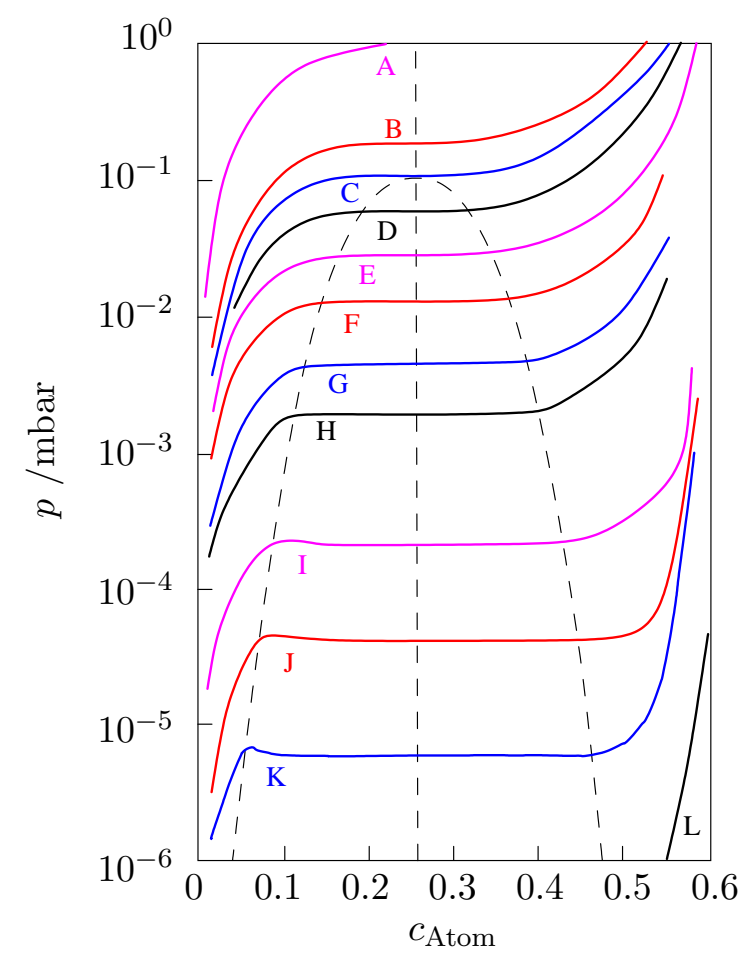

Abbildung 2.4: Isotherme für bulk Niob-Wasserstoff [PT69]. Für unterschiedliche Temperaturen wurden von J. A. Pryde und C. G. Titcomb Isothermen bestimmt. Die hier dargestellten wurden bei den Temperaturen A: $274{ }^{\circ} \mathrm{C}, \mathrm{B}: 230{ }^{\circ} \mathrm{C}, \mathrm{C}: 216{ }^{\circ} \mathrm{C}$, D: $200{ }^{\circ} \mathrm{C}, \mathrm{E}: 186{ }^{\circ} \mathrm{C}, \mathrm{F}: 170{ }^{\circ} \mathrm{C}, \mathrm{G}$ : $147{ }^{\circ} \mathrm{C}, \mathrm{H}: 133{ }^{\circ} \mathrm{C}, \mathrm{I}: 99^{\circ} \mathrm{C}, \mathrm{J}: 79^{\circ} \mathrm{C}$, $\mathrm{K}: 59{ }^{\circ} \mathrm{C}$ und L: $22{ }^{\circ} \mathrm{C}$ aufgenommen. Der Plateaudruck der Isotherme L beträgt $2.4 \cdot 10^{-7} \mathrm{mbar}$.

[PT69]). Bei diesen Isothermen tritt ein Plateaudruck auf, bei dem die Umwandlung von der $\alpha$-Phase in das Hydrid stattfindet. Im massiven System findet diese Umwandlung bei einem festen Druck statt, d.h. sobald ein bestimmter Druck erreicht wird, bildet sich in der kompletten Probe das Hydrid (GIBBssche Phasenregel). Unterhalb des Plateaudrucks entsteht kein Hydrid. Dieser Plateaudruck hängt exponentiell von der Temperatur ab (vgl. J. A. Pryde und C. G. Tiтcomb). Aus den Daten von Abb. 2.4 wurde der Zusammenhang

$$
p_{\text {plateau }}=1.33 \cdot 10\left\{7.87(14)-\frac{82.5(11) \mathrm{kJ}}{2.303 R T}\right\} \text { mbar }
$$

zwischen Temperatur und Plateaudruck bestimmt [PT69]. Ein Ausschnitt dieser Abhängigkeit von der Temperatur ist in Abb. 2.5 dargestellt. Bei einer Temperatur von $20{ }^{\circ} \mathrm{C}$ findet die Umwandlung von der $\alpha$-Phase in das Hydrid bei einem Druck von $1.9 \cdot 10^{-7}$ mbar statt.

\subsection{Dünne Niob-Wasserstoff-Schichten}

In dünnen Schichten wird das System durch die Reduzierung der Schichtdicke verändert. Hinzu kommen Wechselwirkungen zwischen der dünnen Schicht und dem Substrat, auf dem die Proben aufgebracht werden. Die Schicht ist nicht mehr in allen drei Dimensionen frei, sondern in zwei Dimensionen an das Substrat gebunden. Dieser Effekt der endlichen Schichtdicke und Wechselwirkungen mit dem Substrat verursachen eine Verschiebung der Phasengrenzen [Lau98, Dor02]. 


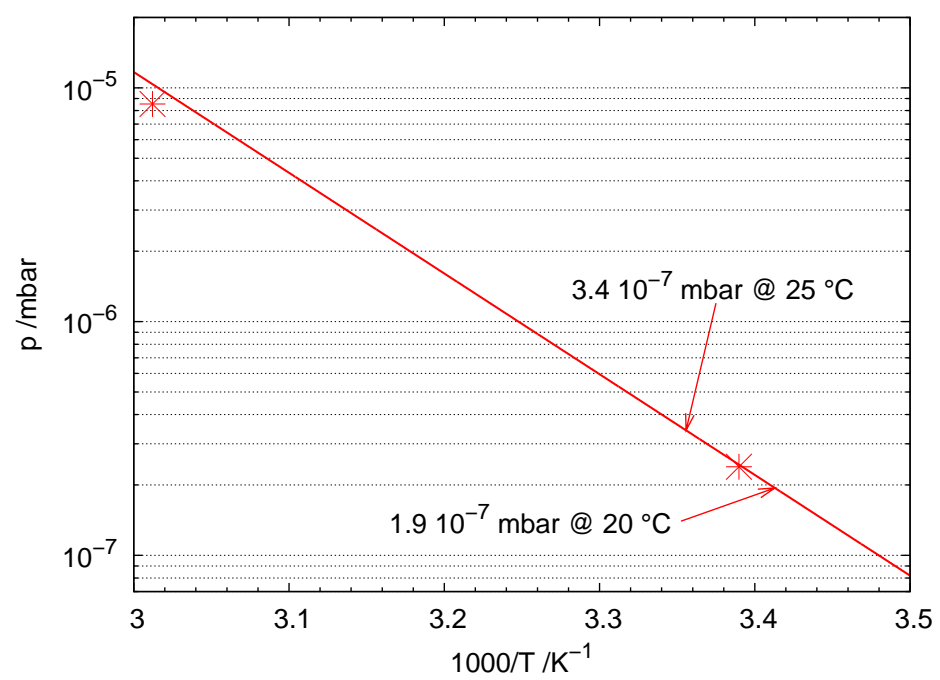

Abbildung 2.5: Plateaudruck der Hydridbildung für massives Niob [PT69]. Gezeigt ist der für diese Arbeit interessante Ausschnitt aus dem Arrheniusplot der Daten von Abbildung 2.4.

\subsubsection{Phasendiagramm}

Die beschriebenen Änderungen der Phasengrenze werden wie folgt gefunden: Die Randlöslichkeit in der $\alpha$-Phase nimmt mit steigender Schichtdicke zu. Die $(\alpha+\beta, \beta)$ Phasengrenze wird veringert [Dor02]. Durch diese beiden Veränderungen unterscheidet sich die Breite der $(\alpha+\beta)$-Mischungslücke für dünne Filme von dem Wert für massive Proben. M. DornheIM gibt für epitaktische Nb-Filme die folgenden Werte für die Breite des Koexistenzbereiches an [Dor02]:

$$
\begin{aligned}
\Delta c_{100 \mathrm{~nm}} & \approx 0.55 \\
\Delta c_{35 \mathrm{~nm}} & \approx 0.4 \\
\Delta c_{25 \mathrm{~nm}} & \approx 0.3
\end{aligned}
$$

\subsubsection{Gitterdehnung}

Auch die Gitterausdehnung bei Wasserstoffbeladung verändert sich drastisch, da sich die Schicht nicht in alle drei Dimensionen frei ausdehnen kann. In der Schichtebene (in-plane) ist die Dehnung durch die Substrat-Schicht-Bindung nicht möglich. Um die Ausdehnung zu berechnen, wird folgendes Gedankenexperiment durchgeführt $\left[\mathrm{LFK}^{+}\right.$99]:

Wird ein Film ohne Substrat mit Wasserstoff beladen, kommt es zu einer gleichmäßigen Ausdehnung $\varepsilon_{0}$ in alle drei Raumrichtungen $\left(\varepsilon_{x x}^{0}=\varepsilon_{y y}^{0}=\varepsilon_{z z}^{0}=\varepsilon_{0}\right)$, wie es in Abb 2.6 illustriert ist. Um wieder auf die ursprüngliche Größe in $x$ - und $y$ Richtung zu kommen, wird eine zusätzliche Dehnung $\left(\varepsilon_{x x}, \varepsilon_{y y}\right)$ von $-\varepsilon_{0}$ benötigt. Dies führt zu mechanischen Spannungen in $x$ - und $y$-Richtung, sowie zu einer zusätzlichen 

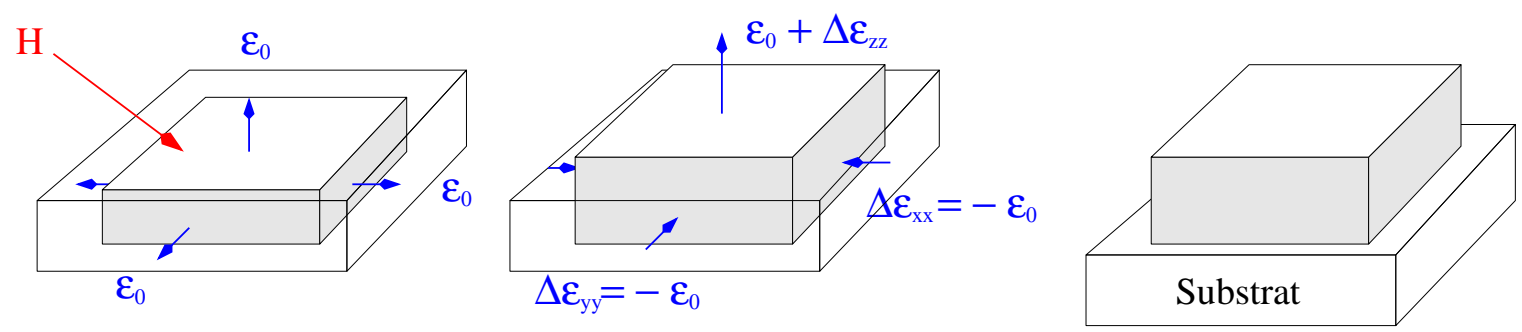

Abbildung 2.6: Modell zur Beschreibung der Dehnung in einem mit Wasserstoff beladenen dünnen Film. Eine freie Probe dehnt sich in alle drei Raumrichtungen aus. Da bei einer auf einem Substrat befindlichen Schicht keine in-plane Dehnung zugelassen ist, treten inplane Spannungen auf und outof-plane wird der Film stärker als die freie Probe gedehnt.

Ausdehnung in $z$-Richtung um $\Delta \varepsilon_{z z}$, die sich aus den elastischen Konstanten $C_{i j}$ des Materials ergibt. Diese Werte sind von K. H. Hellwege und O. Madelung [HM84] zusammengestellt worden. Die Ausdehnung in z-Richtung berechnet sich für kubische Kristalle nach Q. M. YANG et.al. [YSF ${ }^{+}$96]:

$$
\varepsilon_{z z}=\varepsilon_{z z}^{0}+\Delta \varepsilon_{z z}=\left(1+\frac{C_{11}+3 C_{12}-2 C_{44}}{C_{11}+C_{12}+2 C_{44}}\right) \varepsilon_{0}
$$

Nun hat der Film wieder die ursprüngliche Ausdehnung in $x$ - und $y$-Richtung sowie eine größere Ausdehnung in $z$-Richtung und kann im Gedankenexperiment wieder auf das Substrat gebracht werden. Auf die in der Filmebene auftretende Spannung wird in Abschnitt 2.2.4 eingegangen. Aus Gl. (2.1) und Gl. (2.3) erhält man für die isotrope Dehnung durch die Wasserstoffbeladung

$$
\varepsilon_{0}=0.058 \cdot c_{\mathrm{H}}
$$

und dadurch ergibt sich eine vertikale Ausdehnung von:

$$
\varepsilon_{z z}=\frac{\Delta z}{h_{0}}=0.136 \cdot c_{\mathrm{H}}
$$

wie es bei U. LAUDAHN et.al. $\left[\mathrm{LPB}^{+} 99\right]$ nachzulesen ist.

\subsubsection{Thermodynamik}

In dünnen Schichten ist die Thermodynamik im Vergleich $\mathrm{zu}$ massiven Proben verändert (s. Abschnitt 2.1.3). Abb. 2.7 zeigt den Verlauf der elektromotorischen Kraft (EMK) einer elektrolytisch mit Wasserstoff beladenen $100 \mathrm{~nm}$ dünnen polykristallinen Niobschicht in Abhängigkeit von der Wasserstoffkonzentration. Aus der elektromotorischen Kraft $U$ wird anhand der Formel $\mu=\mu_{0, U}+n \cdot F \cdot U$ das elektrochemische Potential $\mu$ bestimmt. Als zweite $y$-Achse ist der Wasserstoffdruck, der dem elektrochemischen Potential entspricht, dargestellt. Die Umrechnung erfolgt durch die Gleichungen $\mu_{p}=\mu_{0, p}+R \cdot T \cdot \log p / p_{0}$ und $\mu_{p} / 2=\mu_{U}$. Der Plateaudruck [AV78b] 


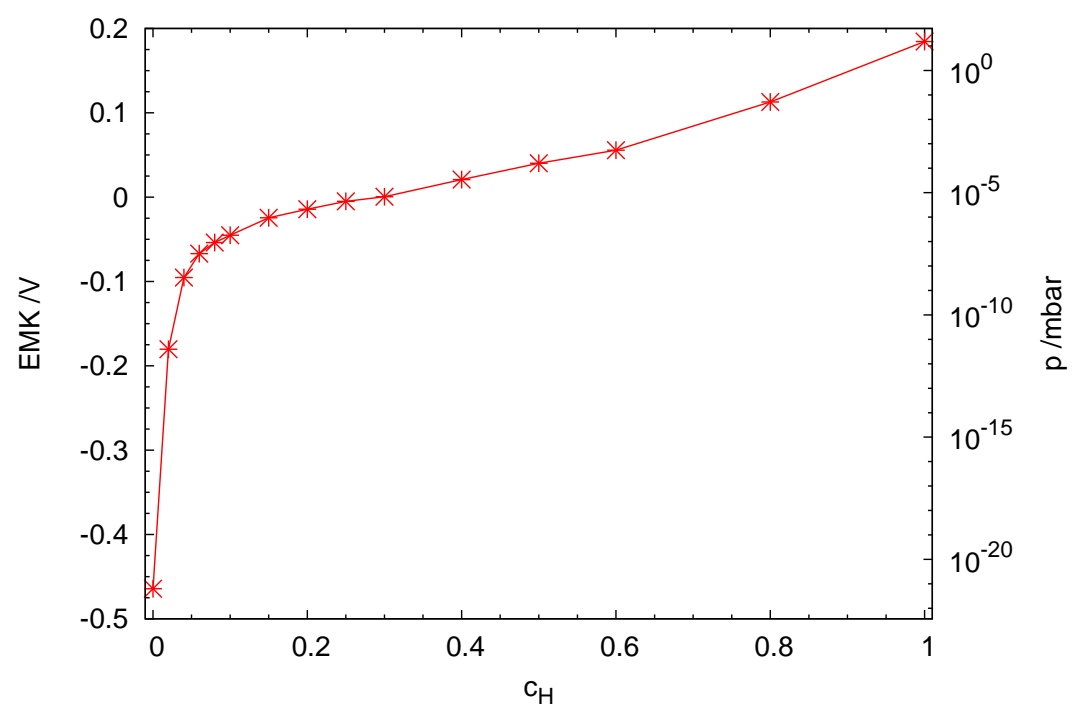

Abbildung 2.7: EMK einer $100 \mathrm{~nm}$ dünnen Nb-Schicht in Abhängigkeit von der Wasserstoffkonzentration gemessen mit einer $\mathrm{Ag} / \mathrm{AgCl} / \mathrm{Cl}^{-}$Elektrode. Der Nb-Film wurde für die elektrochemische Beladung mit einer Pd-Schicht bedeckt*. Das chemische Potential steigt mit der Wasserstoffkonzentration streng monoton an. Die Daten wurden von E. Nikitin zur Verfügung gestellt [Nik06].

und das elektrochemische Potential [MK87] (gemessen mit einer Kalomel-Elektrode [Kir]) sind von der Phasenumwandlung von Palladium bekannt. So können die Konstanten $\mu_{0, U}, \mu_{0, p}$ und $p_{0}$ festgelegt werden. Es wird in Abb. 2.7 deutlich, dass das chemische Potential streng monoton mit der Wasserstoffkonzentration ansteigt. Bei der stufenweisen Beladung der Schicht lagen die Beladungsschritte ca. 5 min auseinander [Nik06]. Aus diesem Grund kann der Anstieg des chemischen Potentials mit der Konzentration auch kinetisch begründet sein. Eine genaue Überprüfung der Gleichgewichtsbedingung bei einer Beladung über einen langen Zeitraum wird in Abschnitt 4.3.4 beschrieben.

\subsubsection{Mechanische Spannungen in haftenden Schichten}

Da bei Niob die elastischen Eigenschaften nicht isotrop sind und in dieser Arbeit epitaktische Schichten behandelt werden, soll hier kurz auf die Richtungsabhängigkeit des Elastizitätsmoduls und der Poissonzahl eingegangen werden. Eine detaillierte Beschreibung kann dem Artikel von D. SANDER [San99] oder dem Lehrbuch von W. Voigt [Voi28] entnommen werden. Ausgegangen wird vom Zusammenhang zwischen der Spannung und der Dehnung über den Tensor der Steifigkeiten $\left(S_{i j k l}\right)$. Dieser Tensor 4. Stufe hat 81 Komponenten, von denen aus Symmetriegründen

*Bei einem Nb-Pd-Schichtsystem wird der Wasserstoff zum Großteil im Niob gelöst. Im Gleichgewicht beträgt der Konzentrationsunterschied $\frac{c_{\mathrm{H}, \mathrm{Pd}}}{c_{\mathrm{H}, \mathrm{Nb}}}=3 \cdot 10^{-5}$ [Kes00], womit der Einfluss des Palladiums vernachlässigt werden kann. 
in kubischen Gittern nur vier unabhängig voneinander sind. In VoIGTscher Notation [Voi28, Sch74, AM76] ist der Tensor nachfolgend zusammen mit der Elastizitätsmodulmatrix $C_{i j}$ dargestellt

$$
\begin{aligned}
& \sigma=C_{i j} \cdot \varepsilon \\
& C_{i j}^{\text {kubisch }}=\left(\begin{array}{cccccc}
C_{11} & C_{12} & C_{12} & 0 & 0 & 0 \\
C_{12} & C_{11} & C_{12} & 0 & 0 & 0 \\
C_{12} & C_{12} & C_{11} & 0 & 0 & 0 \\
0 & 0 & 0 & C_{44} & 0 & 0 \\
0 & 0 & 0 & 0 & C_{44} & 0 \\
0 & 0 & 0 & 0 & 0 & C_{44}
\end{array}\right) \\
& \varepsilon=S_{i j} \cdot \sigma \\
& S_{i j}^{\text {kubisch }}=\left(\begin{array}{cccccc}
S_{11} & S_{12} & S_{12} & 0 & 0 & 0 \\
S_{12} & S_{11} & S_{12} & 0 & 0 & 0 \\
S_{12} & S_{12} & S_{11} & 0 & 0 & 0 \\
0 & 0 & 0 & S_{44} & 0 & 0 \\
0 & 0 & 0 & 0 & S_{44} & 0 \\
0 & 0 & 0 & 0 & 0 & S_{44}
\end{array}\right) .
\end{aligned}
$$

Der Elastizitätsmodul in Richtung der Koordinatenachsen ist durch den Kehrwert von $S_{11}$ gegeben. Wenn der Modul in anderen Richtungen bestimmt werden soll, muss das Koordinatensystem gemäß

$$
S_{i j k l}^{\prime}=a_{i m} a_{j n} a_{k o} a_{l p} S_{m n o p} \quad i, j, \cdots p=1,2,3
$$

transformiert werden, mit der Transformationsmatrix $a_{i j}$, die die Koordinatensysteme ineinander überführt. Hierbei ist

$$
a_{i j}=\left(\begin{array}{ccc}
\sin \theta \cos \phi & \cos \theta \cos \phi & -\sin \phi \\
\sin \theta \sin \phi & \cos \theta \sin \phi & \cos \phi \\
\cos \theta & -\sin \theta & 0
\end{array}\right)
$$

für eine Drehung mit dem Winkel $\theta$ zur $z$-Achse $(0 \leq \theta<\pi)$ und dem Winkel $\phi$ in der $x$ - $y$-Ebene $(0 \leq \phi<2 \pi)$. Der Elastizitätsmodul $E^{\prime}$ und die Poissonzahl $\nu^{\prime}$ in dem neuen Koordinatensystem in Abhängigkeit von den Winkeln $\theta$ und $\phi$ wird zu

$$
\begin{aligned}
\frac{1}{E^{\prime}} & =S_{11}^{\prime}=S_{1111}^{\prime}=a_{1 m} a_{1 n} a_{1 o} a_{1 p} S_{\text {mnop }} \\
\frac{1}{E^{\prime}} & =S_{11}-2\left(S_{11}-S_{12}-\frac{1}{2} S_{44}\right)\left(a_{11}^{2} a_{12}^{2}+a_{11}^{2} a_{13}^{2}+a_{12}^{2} a_{13}^{2}\right) \\
\nu^{\prime} & =-\frac{S_{12}^{\prime}}{S_{11}^{\prime}}, \\
\nu^{\prime} & =-\frac{S_{12}+2\left(S_{11}-S_{12}-\frac{1}{2} S_{44}\right)\left(a_{11}^{2} a_{21}^{2}+a_{12}^{2} a_{22}^{2}+a_{13}^{2} a_{23}^{2}\right)}{S_{11}-2\left(S_{11}-S_{12}-\frac{1}{2} S_{44}\right)\left(a_{11}^{2} a_{12}^{2}+a_{11}^{2} a_{13}^{2}+a_{12}^{2} a_{13}^{2}\right)}
\end{aligned}
$$


Die Steifigkeiten $S_{i j}$ sind z. B. bei K. H. Hellwege und O. Madelung angegeben [HM84] und sind für Niob: $S_{11}=6.56 \mathrm{TPa}^{-1}, S_{12}=-2.29 \mathrm{TPa}^{-1}$ und $S_{44}=35.2 \mathrm{TPa}^{-1}$. In Abb. 2.8 ist der richtungsabhängige Elastizitätsmodul und die Poissonzahl dargestellt. Das absolute Maximum des E-Moduls liegt entlang der $\langle 001\rangle$ Richtung, das Minimum des E-Moduls wird in den $\langle 111\rangle$-Richtungen erreicht. In den $\langle 110\rangle$-Richtungen tritt ein lokales Maximum auf, wie in der Schnittdarstellung in der (110)-Ebene zu sehen ist 2.8(b).

Die im Film auftretende Spannung lässt sich als Ableitung des elastischen Anteils der freien Energie $f_{\text {elastisch }}$ nach der Dehnung $\varepsilon_{i}$ berechnen. Der elastische Anteil der freien Energie berechnet sich aus:

$$
f_{\text {elastisch }}=\frac{1}{2} C_{i j k l} \varepsilon_{i j} \varepsilon_{k l}
$$

In kubischen Kristallen ergibt sich somit in der VoigTschen Notation [Voi28, Sch74, AM76]:

$$
f_{\text {elas. }}=\frac{1}{2} C_{11}\left(\varepsilon_{1}^{2}+\varepsilon_{2}^{2}+\varepsilon_{3}^{2}\right)+C_{12}\left(\varepsilon_{1} \varepsilon_{2}+\varepsilon_{1} \varepsilon_{3}+\varepsilon_{2} \varepsilon_{3}\right)+\frac{1}{2} C_{44}\left(\varepsilon_{4}^{2}+\varepsilon_{5}^{2}+\varepsilon_{6}^{2}\right) .
$$

Um die Spannungen in einem (110)-texturierten Film zu berechnen, ist das Koordinatensystem so zu transformieren, dass die neue $x^{\prime}-y^{\prime}$-Ebene der Filmoberfläche entspricht. Diese Transformation entspricht einer Drehung um $\theta=-90^{\circ}$ um die $x$-Achse und einer Drehung um $\phi=-45^{\circ}$ um die $z$-Achse. Die Transformationsmatrix $a_{i j}$ ist nach Gl. (2.16):

$$
a_{i j}=\left(\begin{array}{ccc}
\frac{-1}{\sqrt{2}} & 0 & \frac{1}{\sqrt{2}} \\
\frac{1}{\sqrt{2}} & 0 & \frac{1}{\sqrt{2}} \\
0 & 1 & 0
\end{array}\right) \quad .
$$

Diese Matrix führt das Kristallkoordinatensystem in das Probenkoordinatensystem über. Mit der Tensortransformation erhält man aus der Dehnung im Probenkoordinatensystem $\epsilon$ die Dehnung in den Kristallkoordinaten $\varepsilon$. Wenn keine Scherung im Probenkoordinatensystem auftritt $\left(\epsilon_{4}=\epsilon_{5}=\epsilon_{6}=0\right)$, ist die Dehnung im Kristallkoordinatensystem wie folgt:

$$
\begin{aligned}
\varepsilon_{i j} & =a_{k i} \epsilon_{k l} a_{l j} \\
\varepsilon_{i j} & =\left(\begin{array}{ccc}
\frac{1}{2}\left(\epsilon_{1}+\epsilon_{3}\right) & \frac{1}{2}\left(\epsilon_{3}-\epsilon_{1}\right) & 0 \\
\frac{1}{2}\left(\epsilon_{3}-\epsilon_{1}\right) & \frac{1}{2}\left(\epsilon_{1}+\epsilon_{3}\right) & 0 \\
0 & 0 & \epsilon_{2}
\end{array}\right) .
\end{aligned}
$$

Mit Gl. (2.20) erhält man die freie Energie in Abhängigkeit von den Dehnungen in Probenkoordinaten:

$$
\begin{aligned}
f_{\text {elastisch }}= & \frac{1}{4} C_{11}\left(\epsilon_{1}^{2}+2 \epsilon_{2}^{2}+2 \epsilon_{1} \epsilon_{3}+\epsilon_{3}^{2}\right)+\frac{1}{4} C_{12}\left(\epsilon_{1}^{2}+4 \epsilon_{1} \epsilon_{2}+2 \epsilon_{1} \epsilon_{3}+4 \epsilon_{2} \epsilon_{3}+\epsilon_{3}^{2}\right) \\
& 2 C_{44}\left(\epsilon_{1}^{2}-2 \epsilon_{1} \epsilon_{3}+\epsilon_{3}^{2}\right) .
\end{aligned}
$$




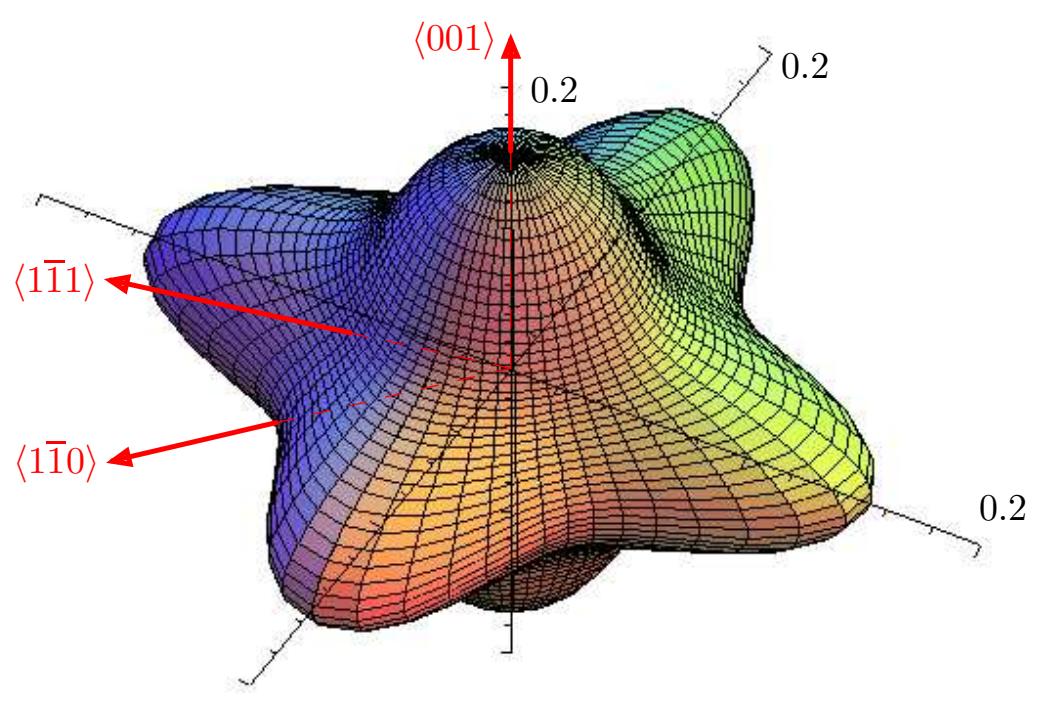

(a) dreidimensional

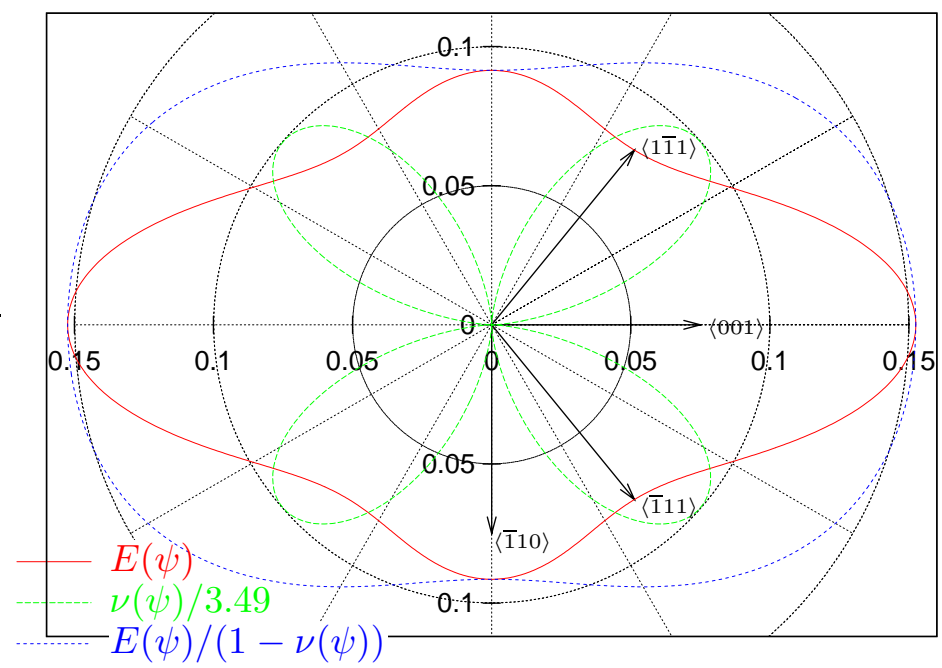

(b) in der (110) Ebene

Abbildung 2.8: Richtungsabhängiger Elastizitätsmodul von Niob. In (a) ist die dreidimensionale Richtungsabhängigkeit dargestellt, in (b) ist eine Darstellung des Moduls sowie der Poissonzahl in der (110)-Ebene zu sehen. Hier sind zusätzlich noch die kristallographischen Richtungen eingetragen. In beiden Graphen ist der E-Modul in TPa aufgetragen. 
Da in $z$-Richtung keine Spannung auftritt $\left(\sigma_{3}=\frac{\partial f_{\text {elastisch }}}{\partial \epsilon_{3}}=0\right)$, berechnen sich die Spannungen im Film in $x$ - und $y$-Richtung zu:

$$
\begin{aligned}
\sigma_{1} & =\frac{\partial f_{\text {elastisch }}}{\partial \epsilon_{1}}=\frac{4\left(C_{11}+C_{12}\right) C_{44} \epsilon_{1}+4 C_{12} C_{44} \epsilon_{2}}{C_{11}+C_{12}+2 C_{44}} \\
\sigma_{2} & =\frac{\partial f_{\text {elastisch }}}{\partial \epsilon_{2}}=\frac{4 C_{12} C_{44} \epsilon_{1}+\left(C_{11}^{2}-2 C_{12}^{2}+C_{11}\left(C_{12}+2 C_{44}\right)\right) \epsilon_{2}}{C_{11}+C_{12}+2 C_{44}} .
\end{aligned}
$$

Mit den elastischen Konstanten für Niob $\left(C_{11}=246 \mathrm{GPa}, C_{12}=132.6 \mathrm{GPa}\right.$ und $\left.C_{44}=27.9 \mathrm{GPa}\right)$ aus [HM84] und einer isotropen in-plane Dehnung $\left(\epsilon_{1}=\epsilon_{2}=-\varepsilon_{0}\right)$ erhält man mit Gl. (2.9) (s. S. 8):

$$
\begin{aligned}
& \sigma_{1=\langle\overline{1} 10\rangle}=-7.62 \mathrm{GPa} \cdot c_{\mathrm{H}}, \\
& \sigma_{2=\langle 001\rangle}=-11.55 \mathrm{GPa} \cdot c_{\mathrm{H}} .
\end{aligned}
$$

Spannungen in der wie hier angegebenen Größenordnung werden in Experimenten bestätigt. U. LAUDAHN beobachtet Spannungen von bis $\mathrm{zu}-8.7 \mathrm{GPa} \cdot c_{\mathrm{H}}$ bei laserdeponierten Niobschichten [Lau98]. In epitaktischen Schichten haben U. LAUDAHN et.al. Spannungen von bis $\mathrm{zu}-6.9 \mathrm{GPa} \cdot c_{\mathrm{H}}$ beobachtet $\left[\mathrm{LPB}^{+} 99\right]$.

\subsubsection{Plastische Verformung}

Aufgrund dieser großen Spannung treten bei hoher Wasserstoffbeladung plastische Verformungen auf. T. ScHOBER hat in massiven Niob beobachtet, dass von Hydridausscheidungen Versetzungsringe in $\langle 111\rangle$-Richtung emitiert werden [SW78]. Für dünne Schichten wird berichtet, dass Versetzungsringe und Misfitversetzungen bei der Beladung mit Wasserstoff auftreten $\left[\mathrm{PGB}^{+} 00\right.$, NKP03]. Da mit einem STM die Versetzungslinien nicht direkt gesehen werden können, sondern nur deren Auswirkungen auf der Oberfläche, soll hier kurz beschrieben werden, welche Einflüsse eine Versetzung in der Schicht auf die Oberfläche hat. In Abb. 2.9 ist schematisch dargestellt, wie kleine inkohärente Ausscheidungen und Misfitversetzungen zu unterscheiden sind (nach A. Pundt et.al. $\left[\mathrm{PGB}^{+} 00\right]$ ). Bei kleinen inkohärenten Ausscheidungen werden aufgrund der größeren Gitterkonstante im Hydrid extrinsische Versetzungsringe emitiert. Diese zusätzlichen Gitterebenen werden an der Oberfläche als Inseln sichtbar (s. Abb. 2.9(a)). Bei einem Höhenprofil durch eine solche Insel werden zwei Stufen sichtbar. Wenn Misfitversetzungen entstehen, wird Material entlang einer Gleitebene an die Oberfläche verschoben (in der Abbildung grau hervorgehoben), und es entsteht eine Gleitstufe an der Oberfläche (s. Abb. 2.9(b)). Deshalb entspricht die Stufenhöhe dem Gitterabstand. Von dieser Stufe ausgehend sinkt das Niveau wieder langsam in Form einer Rampe auf die Ursprungshöhe ab. Die kristallographische Orientierung der Gleitstufe bestimmt sich aus den Richtungen der Oberfläche und der verwendeten Gleitebene. Die Gleitstufe entspricht genau der Schnittlinie dieser beiden Ebenen (vgl. Abb. 2.9(b)). Somit ist es möglich an der Oberfläche, die im Volumen erzeugten, Veränderungen durch plastische Verformung zu beobachten. 


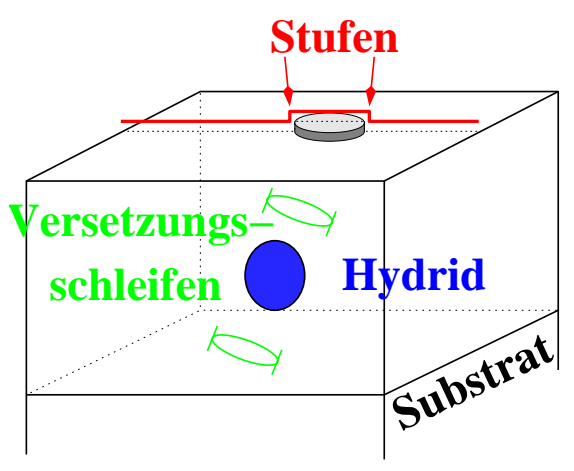

(a) Versetzungsringe

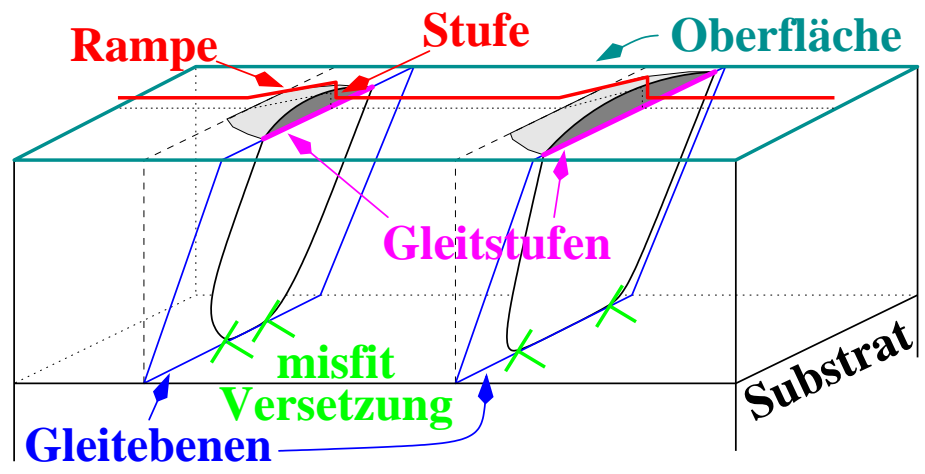

(b) Misfitversetzung

Abbildung 2.9: Einfluss von Versetzungen auf die Oberflächenmorphologie einer dünnen Schicht: Bei Emission von Versetzungsringen durch inkohärente Hydridausscheidungen sind auf der Oberfläche Inseln zu sehen, bei Misfitversetzungen werden an der Oberfläche linienförmige Gleitstufen mit Rampen erwartet $\left[\mathrm{PGB}^{+} 00\right]$.

\subsection{Diffusion in dünnen Schichten}

In diesem Abschnitt soll kurz beschrieben werden, in welchem Zeitraum sich der Wasserstoff, der auf der Oberfläche bereitgestellt wird, aufgrund der Diffusion in der Schicht gleichmäßig verteilt hat. Die Randbedingungen für die Diffusionsgleichung sind wie folgt (vgl. Abb. 2.10):

- Die Schicht wird als eine in zwei Dimensionen ( $x$-y-Ebene) unendliche Platte angesehen. Die dritte Dimension (z-Richtung) hat die Dicke $h$.

- Die Oberfläche ist dem Wasserstoff ausgesetzt. Somit ist die Konzentration an der Oberfläche konstant, da die Menge, die von der Schicht aufgenommen wird, gegenüber dem zur Verfügung stehenden Wasserstoff im Kammervolumen vernachlässigbar klein ist.

- Vom Substrat wird kein Wasserstoff aufgenommen, d.h. die Substrat-FilmGrenzfläche kann als Spiegelebene für das Problem angesehen werden.

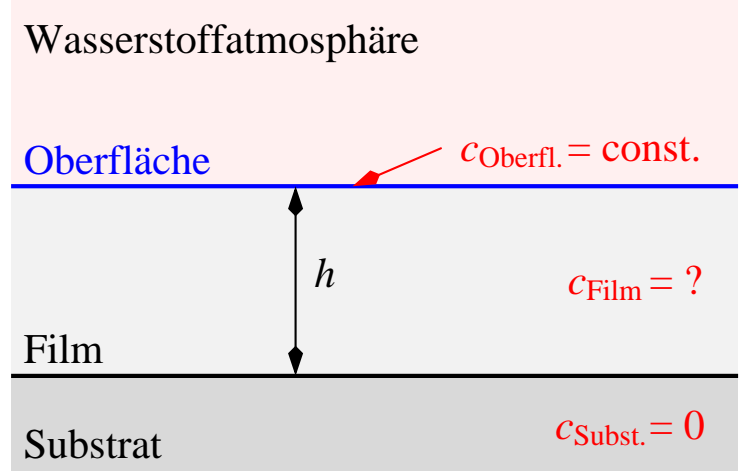

Abbildung 2.10: Randbedingungen für die Lösung der Diffusionsgleichung für dünne Schichten: Die Schicht ist in $x$ und $y$-Richtung unendlich ausgedehnt, die Dicke in $z$-Richtung beträgt $h$. An der Oberfläche ist die Konzentration konstant $\left(c_{\text {Oberfl. }}=\right.$ const. $)$ und in das Substrat kann kein Wasserstoff gelangen $\left(c_{\text {Subst. }}=0\right)$. 
- Der zeitbestimmende Schritt ist die Diffusion. Oberflächenbarrieren bzw. Ratenlimitierung durch die Oberfläche werden vernachlässigt.

Die mathematischen Grundlagen für dieses Problem sind in [Cra75] beschrieben. Der Konzentrationsverlauf in der Probe wird durch die Gleichung

$$
\frac{c(z, t)-c_{\mathrm{s}}}{c_{\infty}-c_{\mathrm{s}}}=1-\frac{4}{\pi} \sum_{n=0}^{\infty} \frac{(-1)^{n}}{2 n+1} \exp \left\{-(2 n+1)^{2} \frac{\pi^{2}}{4} \frac{D t}{h^{2}}\right\} \cos \left\{\frac{2 n+1}{2} \pi \frac{z}{h}\right\}
$$

beschrieben. Den Mittelwert der Konzentration kann man durch Integration entlang der $z$-Achse über die Probendicke im Bereich $[0, h]$ berechnen:

$$
\begin{aligned}
\frac{\bar{c}-c_{\mathrm{s}}}{c_{\infty}-c_{\mathrm{s}}} & =1-\frac{8}{\pi^{2}} \sum_{n=0}^{\infty} \frac{1}{(2 n+1)^{2}} \exp \left\{-(2 n+1)^{2} \frac{\pi^{2}}{4} \frac{D t}{h^{2}}\right\} \\
& =1-\frac{\sum_{n=0}^{\infty} \frac{1}{(2 n+1)^{2}} \exp \left\{-(2 n+1)^{2} \frac{\pi^{2}}{4} \frac{D t}{h^{2}}\right\}}{\sum_{n=0}^{\infty} \frac{1}{(2 n+1)^{2}}} \\
& \approx 1-\exp \left\{-\frac{\pi^{2} D t}{4 h^{2}}\right\} .
\end{aligned}
$$

Der Mittelwert $\bar{c}$ über die Schichtdicke $h$ entlang der $z$-Richtung wird deshalb betrachtet, weil mit der oberflächensensitiven Methode STM die unterschiedliche Schichtdicke in der gesamten Höhe $\Delta z_{\text {total }}$ der Probe gemessen wird. Aufgrund des linearen Zusammenhangs zwischen Konzentration $c$, Schichtdicke $h$ und Höhenänderung $\Delta z$ (vgl. Gl. (2.10), S. 8) gibt das Produkt aus mittlerer Konzentration $\bar{c}$ und Schichtdicke $h$ die Höhenänderung $\Delta z_{\text {total }}$ an, die vom STM gemessen wird. Nach Gleichung (2.29) kann die Zeit, in der $99.9 \%$ des Gleichgewichtswertes erreicht wird, wie folgt berechnet werden:

$$
\begin{aligned}
t_{99.9 \%} & \approx-\frac{4 h^{2}}{\pi^{2} D} \ln \{0.001\} \\
& \approx 2.8 \cdot \frac{h^{2}}{D}
\end{aligned}
$$

Dies ergibt für eine $100 \mathrm{~nm}$ dünne Niobschicht mit der Diffusionskonstante des massiven Systems von $D_{\mathrm{Nb}}=8.1 \cdot 10^{-6} \mathrm{~cm}^{2} / \mathrm{s}$ [QVLW83] eine Zeit von:

$$
t_{99.9 \%} \approx 35 \mu \mathrm{s}
$$

Diese Zeit ist um mehrere Größenordnungen kleiner als die Zeit, in der die STMBilder aufgenommen werden. Wenn während der STM-Aufnahmen Veränderungen auftreten, ist die Diffusion in der Nb-Schicht nicht der limitierende Zeitfaktor. 


\subsection{Wasserstoffbeladung aus der Gasphase}

Die ersten Experimente zur Löslichkeit von Wasserstoff in Metallen wurden mit der Beladung von gasförmigen Wasserstoff durchgeführt [Gra66]. A. SiEverTs hat bei seinen Beladungsexperimenten an Palladium festgestellt, dass eine wurzelförmige Abhängigkeit zwischen dem vorgegebenen äußeren Wasserstoffdruck und der gemessenen Wasserstoffkonzentration im Palladium besteht [Sie29]. Diese Abhängigkeit kann über das chemische Potential in der Gasphase $\mu_{\mathrm{H}_{2}}^{\mathrm{g}}$ und im Metall $\mu_{\mathrm{H}}^{\mathrm{s}}$ hergeleitet werden:

$$
\begin{aligned}
\mu_{\mathrm{H}}^{\mathrm{s}} & =\frac{1}{2} \mu_{\mathrm{H}_{2}}^{\mathrm{g}} \\
\text { Gasphase: } \quad \mu_{\mathrm{H}_{2}}^{\mathrm{g}} & =\mu_{\mathrm{H}_{2}}^{\mathrm{g} 0}+R T \ln \frac{p_{\mathrm{H}_{2}}}{p_{0}} \\
\text { im Metall: } \quad \mu_{\mathrm{H}}^{\mathrm{s}} & =\mu_{\mathrm{H}}^{\mathrm{s} 0}+R T \ln \frac{c}{1-c} \\
\mu_{\mathrm{H}}^{\mathrm{s} 0}+R T \ln \frac{c}{1-c} & =\frac{1}{2}\left(\mu_{\mathrm{H}_{2}}^{\mathrm{g} 0}+R T \ln \frac{p_{\mathrm{H}_{2}}}{p_{0}}\right) .
\end{aligned}
$$

Für eine geringe Konzentrationen $(c \ll 1)$ kann der Bruch $\frac{c}{1-c}$ durch die Konzentration $c$ angenähert werden. Sämtliche Konstanten können in eine temperatur- und materialabhängige Konstante $S$ zusammengefasst werden:

$$
\ln c=\ln S+\frac{1}{2} \ln p_{\mathrm{H}_{2}}
$$

Es ist zu sehen, dass ein wurzelförmiger Zusammenhang zwischen Konzentration $c$ und äußerem Wasserstoffdruck $p_{\mathrm{H}_{2}}$ besteht:

$$
c=S \cdot \sqrt{p_{\mathrm{H}_{2}}} \text {. }
$$

In diesem Kapitel sind einige Grundlangen des Systems Wasserstoff-Niob behandelt worden. Dabei wurde zuerst auf das massive System eingegangen, um dann die bei dünnen Schichten auftretenden Unterschiede zu beschreiben. Die großen mechanischen Spannungen, die in dünnen haftenden Schichten auftreten, wurden ausführlich behandelt. Im folgenden Kapitel werden die eingesetzten experimentellen Methoden beschrieben. 


\section{Experimentelle Methoden}

In diesem Kapitel werden die benutzten Methoden kurz dargestellt und einzelne Aspekte zu den Methoden genauer beschrieben. Zu Beginn werden in Abschnitt 3.1 die eingesetzten Apparaturen beschrieben. Anschließend wird in Abschnitt 3.2 auf die Präparation der Proben sowie deren Charakterisierung eingegangen. Abschnitt 3.3 zeigt, wie mit Hilfe der Finite-Elemente-Methode die Berechnung von Spannung und Dehnung einer Ausscheidung in einer dünnen Schicht durchgeführt werden kann.

\subsection{Eingesetzte Apparaturen}

In diesem Abschnitt werden die für die Messungen verwendeten Apparaturen vorgestellt. Dabei wird auf die Ultrahochvakuumanlage (Abschnitt 3.1.1), die Beladung mit Wasserstoff aus der Gasphase in der UHV-Anlage (Abschnitt 3.1.2) und die Rastertunnelmikroskopie (Abschnitt 3.1.3) behandelt.

\subsubsection{UHV-Anlage}

Für die Probenherstellung, sowie die Wasserstoffbeladung und gleichzeitige STMMessung wurde eine UHV-Anlage mit einem Basisdruck $p<3 \cdot 10^{-10}$ mbar verwendet. Bei der Anlage handelt es sich um ein Dreikammersystem. Die drei Kammern sind die Schleuse, die Sputterkammer und die STM-Kammer. Eine schematische Darstellung der Anlage ist in Abbildung 3.1 zu sehen. Im linken Bereich sind zwei Magnettransferstangen zu erkennen. Mit der einen ist es möglich, die Substrate bzw. Proben von der Schleuse zum Probenhalter am Manipulator, der sich in der Mitte befindet, zu transportieren. Mit dem Manipulator kann die Probe in $x-, y$ - und $z$-Richtung verschoben werden, womit eine optimale Position über dem Targetwürfel eingestellt wird. Auf dem Targetwürfel sind vier verschiedene Targets montiert. Durch Rotation um $90^{\circ}$ ist es möglich, die unterschiedlichen Materialien zu wählen. Diese Anordnung ermöglicht schnell zwischen verschiedenen Metallen zu wechseln. So kann zunächst mit einem Niobtarget eine Schicht erstellt werden und anschließend mit einem Palladiumtarget eine Deckschicht aufgebracht werden. Die Probenherstellung wird in Abschnitt 3.2 ausführlich beschrieben. Im Manipulator ist eine Heizung integriert, mit der die Proben auf bis zu $850^{\circ} \mathrm{C}$ erhitzt werden können. Durch eine Wasserkühlung wird diese hohe Temperatur auf einen kleinen Bereich um die Probe beschränkt. Beim Ausschalten der Heizung wird über die Kühlung die Wärme abgeführt. Das Thermoelement ist ca. $1 \mathrm{~cm}$ von der Probe entfernt am Manipulator angebracht. Eine Messung durch 


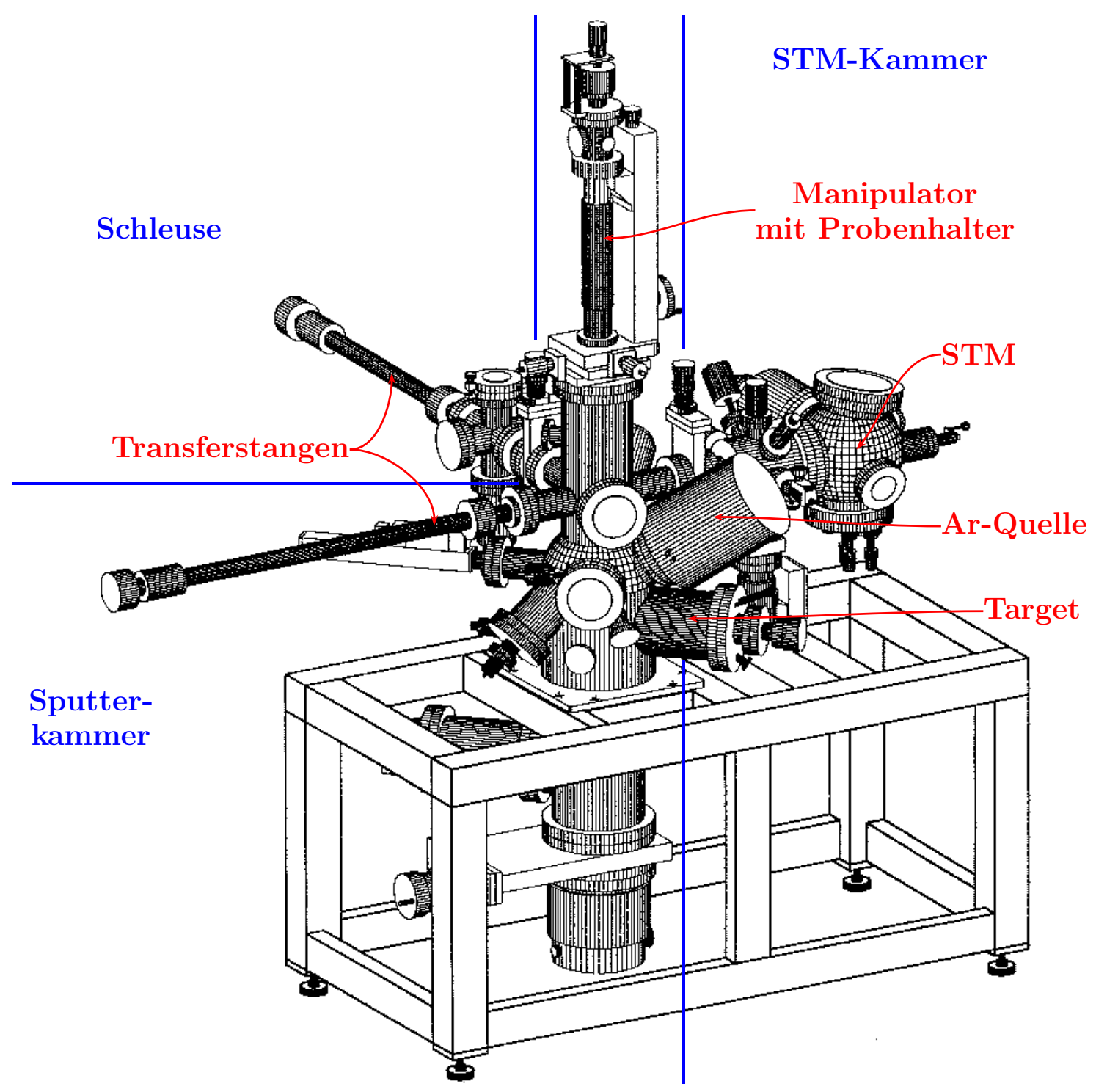

Abbildung 3.1: Schematische Darstellung der eingesetzten UHV-Kammer. Die Kammer besteht aus drei Teilen, die durch Plattenventile voneinander getrennt werden können. Diese Teile sind die Schleuse, die Sputterkammer und die STM-Kammer. Die Grenzen zwischen diesen Bereichen sind in der Darstellung durch blaue Linien hervorgehoben. Mit den Transferstangen werden die Proben von einem zum anderen Kammerteil verschoben. In der Sputterkammer wird die Probe durch den Manipulator in alle drei Raumrichtungen bewegt. Mit einem Wobblestick wird die Probe in das STM eingebaut und kann in diesem Kammerteil auch in einem Regal zwischengelagert werden.

ein weiteres Thermoelement an der Probenposition ergab einen Fehler von weniger als $10^{\circ} \mathrm{C}$ bei einer Temperatur von $850^{\circ} \mathrm{C}$ [Zep]. Nach der Schichtherstellung wird die Probe mit der zweiten Transferstange in die STM-Kammer geschleust. In die- 


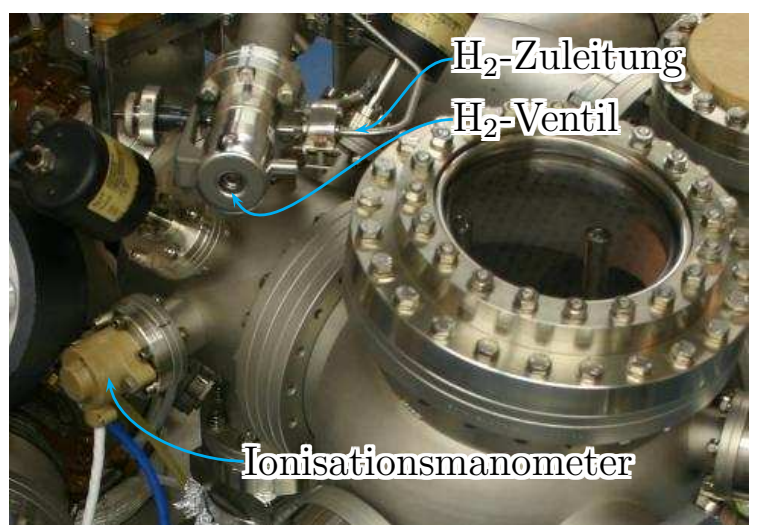

(a) Aufbau

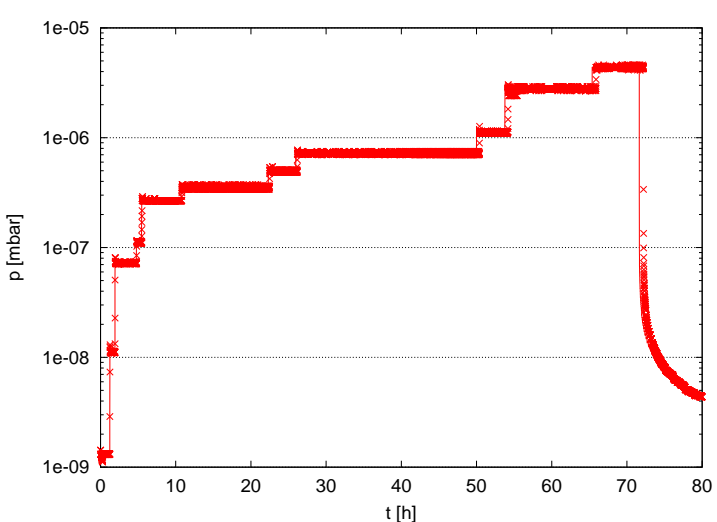

(b) Druckverlauf

Abbildung 3.2: Experimenteller Aufbau der Gasbeladung und Druckverlauf während einer Beladung. In der Mitte des linken Bildes ist das Nadelventil mit dem Wasserstoffanschluss über dem Edelstahlrohr zu sehen. Die Mengenzugabe wird elektronisch auf einen konstanten Druck kontrolliert. Im rechten Graphen ist die stufenweise Druckerhöhung der einzelnen Beladungsschritte erkennbar.

ser Kammer wird die Wasserstoffbeladung aus der Gasphase in einem Druckbereich von $1 \cdot 10^{-9}$ mbar bis 0.5 bar mit gleichzeitiger Rastertunnelmikroskopie durchgeführt. Auf die Eigenschaften der Rastertunnelmikroskopie wird in Abschnitt 3.1.3 eingegangen. Im Abschnitt 3.1.2 werden die Aspekte der Gasbeladung beschrieben. Beide Teile der Kammer können durch ein Ventil voneinander getrennt werden, so dass eine Druckerhöhung in einem Kammerteil keine Veränderung des Drucks im anderen Kammerteil hervorruft.

\subsubsection{Gasbeladung}

Nachdem in Abschnitt 2.4 (s. S. 16) der theoretische Zusammenhang zwischen dem äußeren Wasserstoffdruck und der Wasserstoffkonzentration im Metall erläutert worden ist, wird die experimentelle Durchführung der Beladung beschrieben, die in einer UHV-Kammer abläuft. Sie ist in einem Druckbereich zwischen $10^{-9}$ mbar und 0.5 bar möglich, wobei in dem Fenster von $10^{-3}$ mbar bis 10 mbar aufgrund der Gasentladung das Rastertunnelmikroskop nicht betrieben werden kann [Omi98]. Während der Beladung läuft die Turbopumpe. Wasserstoff mit einer Reinheit von 5.6 wird über ein Edelstahlrohr zugeleitet und durch ein elektromagnetisch geregeltes Nadelventil in die Kammer eingelassen (vgl. Abb. 3.2(a)). Der Kammerdruck wird durch ein Ionisationsmanometer gemessen und die Durchflussmenge des Nadelventils so geregelt, dass der vorgegebene Druck eingestellt wird. Mit der so konstruierten Lösung ist es möglich, den Druck über eine lange Zeit ohne große Schwankungen konstant zu halten. Der Aufbau und ein Druckverlauf ist in Abb. 3.2(b) sichtbar. Im linken Teil sind die schon beschriebenen Komponenten markiert und im rechten Graphen ist der Druckverlauf 

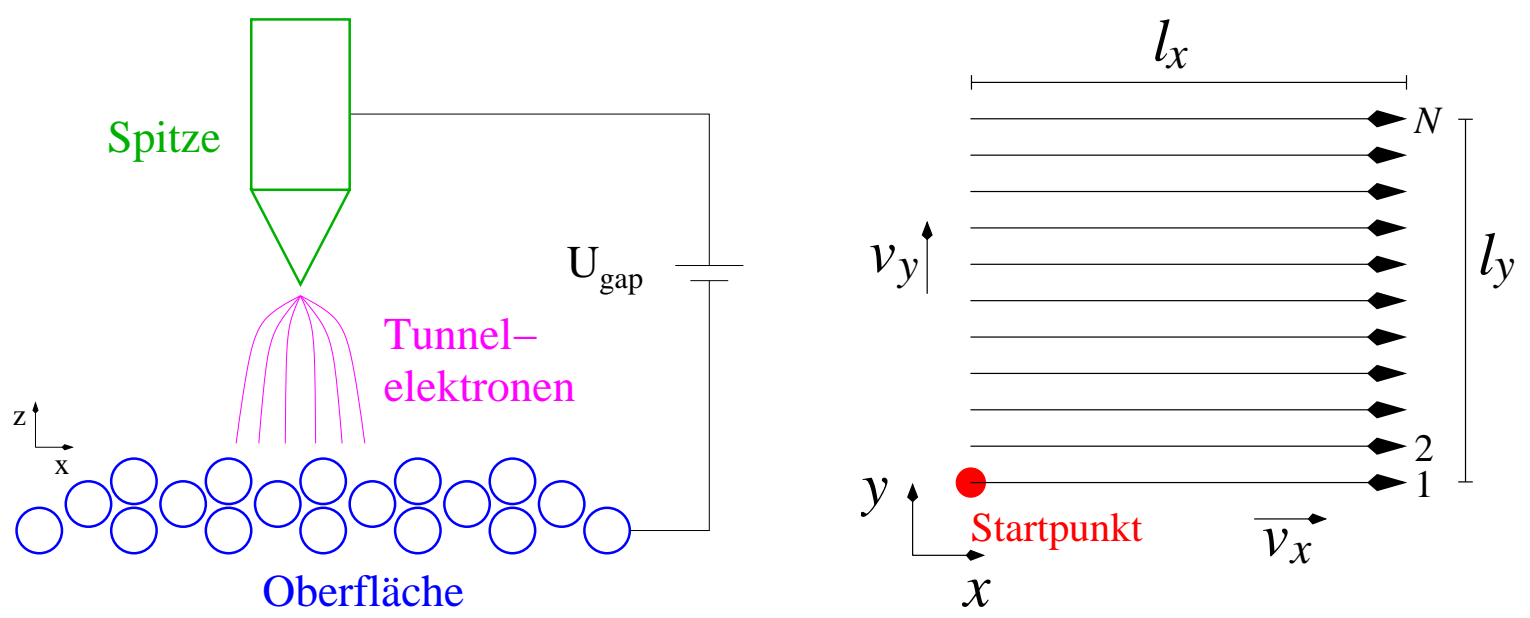

Abbildung 3.3: Grundprinzipien topographischer Rastertunnelmikroskopie. Zwischen Spitze und Probe wird eine Spannung $U_{\text {gab }}$ angelegt. Der Abstand $z$ von Spitze und Probe wird so geregelt, dass der Tunnelstrom $I$ konstant bleibt. Um ein Bild von der Oberfläche zu bekommen, wird die $x-y$-Fläche zeilenweise abgerastert.

einer Wasserstoffbeladung dargestellt. Hier ist deutlich der stufenweise Druckanstieg über einen Zeitraum von $70 \mathrm{~h}$ zu erkennen. Die Stufen werden durch die Veränderung der Druckvorgabe in der Regelelektronik hervorgerufen. Zwischen den vorgegebenen Druckerhöhungen bleibt der Druck konstant. Die Druckverringerung ist durch die größere Trägheit des Systems problematisch für die Steuerung. Diese Regelung ist bis zu einem Druck von $1 \cdot 10^{-4}$ mbar möglich. Oberhalb dieses Druckbereichs kann das Ionisationsmanometer, auf das bei der Regelung zurückgegriffen wird, nicht mehr betrieben werden.

\subsubsection{Rastertunnelmikroskopie}

Bei der Rastertunnelmikroskopie wird mit einer Spitze eine Oberfläche auf atomarer Skala abgerastert, wobei lokale Probeneigenschaften aufgezeichnet (vgl. Abb. 3.3) werden. Der zugrunde liegende physikalische Effekt ist die Messung des Tunnelstroms, der zwischen zwei geladenen Körpern mit geringem Abstand fließt. Um Ladungen zu erzeugen, wird zwischen der Tunnelspitze und der Oberfläche eine Spannung $U_{\text {gap }}$ angelegt. Aufgrund dieser Potentialdifferenz tunneln die Elektronen zwischen der Spitze und der Probe. Die Rastertunnelmikroskopie wurde von G. Binnig, H. Rohrer et.al. [BRGH82] erstmalig vorgestellt. Für diese neue Untersuchungsmethode erhielten G. Binnig und H. Rohrer 1986 den Nobelpreis für Physik. Der Tunnelstrom $I$ wird von der Position $(x, y, z)$ der Spitze beeinflusst. Die Tatsache, dass bei gut präparierten Spitzen nur ein Atom am Apex sitzt, ermöglicht eine atomare Auflösung, weil zwischen dem Abstand $\Delta z$ von Spitze und Probe und dem Tunnelstrom $I$ eine exponentielle 
Abhängigkeit besteht. Um ein STM-Bild zu erhalten, wird die Spitze über die Probe in $x$ - und $y$-Richtung bewegt und eine Fläche zeilenweise abgerastert.

Bei einer STM-Messung werden von den fünf unabhängigen Parametern $x, y, z, I$ und $U_{\text {gap }}$ je nach Messmodus unterschiedliche Parameter vorgegeben, verändert bzw. geregelt. Es wird zwischen dem Spektroskopie- und Topographiemodus unterschieden. Im Spektroskopiebetrieb werden drei Parameter vorgegeben und dann Kurven $I(z)$, $I\left(U_{\text {gap }}\right)$ oder andere Abhängigkeiten wie differentielle Leitfähigkeiten $\left(\frac{\partial I}{\partial U}(U)\right)$ gemessen. Im Topographiemodus wird die Spannung $U_{\text {gap }}$ und der Strom $I$ vorgegeben und der Abstand $z$ so verändert, dass der gemessene Tunnelstrom dem vorgegebenen entspricht. Dieser Abstand $z$ ist dann die aufgenommene Messgröße. Beim Verändern der Position in $x$ - und $y$-Richtung wird eine Fläche mit konstantem Tunnelstrom (bzw. Zustandsdichte) aufgenommen. Durch Abrastern der Probenoberfläche entsteht ein topographisches Bild. Durch die Adsorption von Wasserstoff wird die Oberflächenzustandsdichte verändert. Dies führt zu einer Höhenveränderung beim aufgegenommenen Topographiebild. Bei geringer Wasserstoffbeladung konnten A. PUndT et.al. den Höhenunterschied durch die Adsorption von Wasserstoff an einer Gadoliniumoberfläche sichtbar machen $\left[\mathrm{PGB}^{+} 00\right]$. Die gemessene Höhendifferenz ist kleiner als $0.1 \mathrm{~nm}$. Die Veränderungen der Elektronenzustandsdichte an der Oberfläche durch adsorbierten Wasserstoff hat somit nur einen kleinen Einfluss auf die Höheninformation, die bei einem Topographiebild aufgenommen wird.

Im Abschnitt 3.1.3.1 wird das benutzte Tunnelmikroskop kurz beschrieben. In Abschnitt 3.1.3.2 wird auf die implizite Zeitachse eingegangen. Sie entsteht, weil die einzelnen Messpunkte eines STM-Bildes nacheinander aufgenommen werden.

Weitere Details zur Rastertunnelmikroskopie sind in der Literatur ausführlich dargestellt [SK93, GW92, WG92, Wie94].

\subsubsection{Verwendeter Aufbau}

Die STM-Messungen dieser Arbeit wurden an einem MicroSTM der Firma Omicron durchgeführt. Ein Ausschnitt des Mikroskops mit Scanner, Probe und Aufhängung ist in Abb. $3.4 \mathrm{zu}$ sehen. Die Mikroskopeinheit ist an drei Federn aufgehängt und eine Wirbelstrombremse sorgt für eine starke Dämpfung der Schwingung des Mikroskops für Frequenzen oberhalb der Eigenfrequenz von ca. $2 \mathrm{~Hz}$. Durch diese Konstruktion kann auch bei laufenden Turbo- und Membranpumpen mit dem Mikroskop gearbeitet werden. Die Probe wird kopfüber auf einem speziellen Blech in das STM eingebaut. Der Scanner mit der STM-Spitze nähert sich von unten an die Probe, um diese dann für die Aufnahme abzurastern. Es ist möglich, den Scanner mit Hilfe eines Wobblesticks aus dem Mikroskop herauszunehmen, um dann die Spitze außerhalb der Kammer auszutauschen. Als STM-Spitzen wurden mechanisch gedünnte Pt/Ir (90/10) Drähte verwendet.

Mit dem Mikroskop ist es nicht möglich, im Druckbereich von $10^{-3}$ mbar bis 10 mbar $\mathrm{zu}$ arbeiten, weil dort - entsprechend der Bedienungsanleitung — die Gasentladung auftritt [Omi98]. Hierdurch ergeben sich keine Einschränkungen für die Untersuchun- 


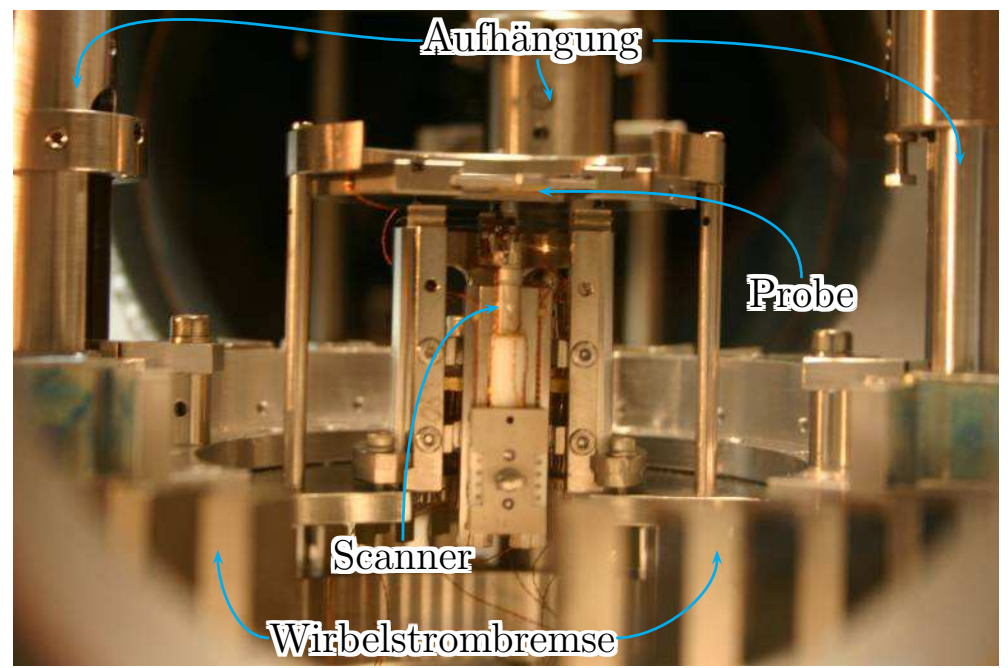

Abbildung 3.4: MicroSTM der Firma Omicron innerhalb der UHV-Kammer. In der Mitte ist der wechselbare Scanner erkennbar, in den die Spitze eingebaut wird. Darüber befindet sich die Probe, die auf einem Blech montiert ist. Diese ganze Einheit ist an drei Federn aufgehängt. Um die Schwingungen der Federaufhängung zu dämpfen, ist eine Wirbelstrombremse angebracht.

gen des Systems Niob-Wasserstoff, da die Phasenumwandlung schon bei geringerem Druck stattfindet.

\subsubsection{Zeitachse in STM-Messungen}

Bei den gemessenen topographischen Oberflächenbildern muss bedacht werden, dass beim Scannen über die Probe Zeit vergeht, und somit, wie in Abb. 3.3 (s. S. 20) dargestellt, die $x$ - und $y$-Richtungen auch Zeitachsen darstellen. Die Umrechnung geschieht über die vorgegebene Scangeschwindigkeit $\left(v_{\text {scan }}\right)$, sowie Anzahl der Zeilen $(N)$ und Zeilenlängen $\left(l_{x}, l_{y}\right)$ der gemessenen Linien. Wenn, wie in Abb. 3.3 skizziert, in allen Zeilen im STM-Bild in die gleiche Richtung gescannt wird und anschließend wieder mit der gleichen Geschwindigkeit an den Zeilenanfang gefahren wird, um die nächste Zeile zu scannen, berechnen sich die Geschwindigkeiten in beide Scannrichtungen wie folgt:

$$
\begin{aligned}
& v_{x}=v_{\text {scan }} \\
& v_{y}=v_{\text {scan }} \frac{l_{y}}{2 \cdot N \cdot l_{x}} .
\end{aligned}
$$

Diese implizite Zeitachse ist dann zu berücksichtigen, wenn sich die Probe während der Messung verändert. Darauf wird in später dargestellten Messungen noch genauer eingegangen. So wird zum Beispiel in Abb. 3.14 (Seite 31), einem Höhenprofil eines STM-Bildes, die $x$-Achse auch als Zeitachse dargestellt. 


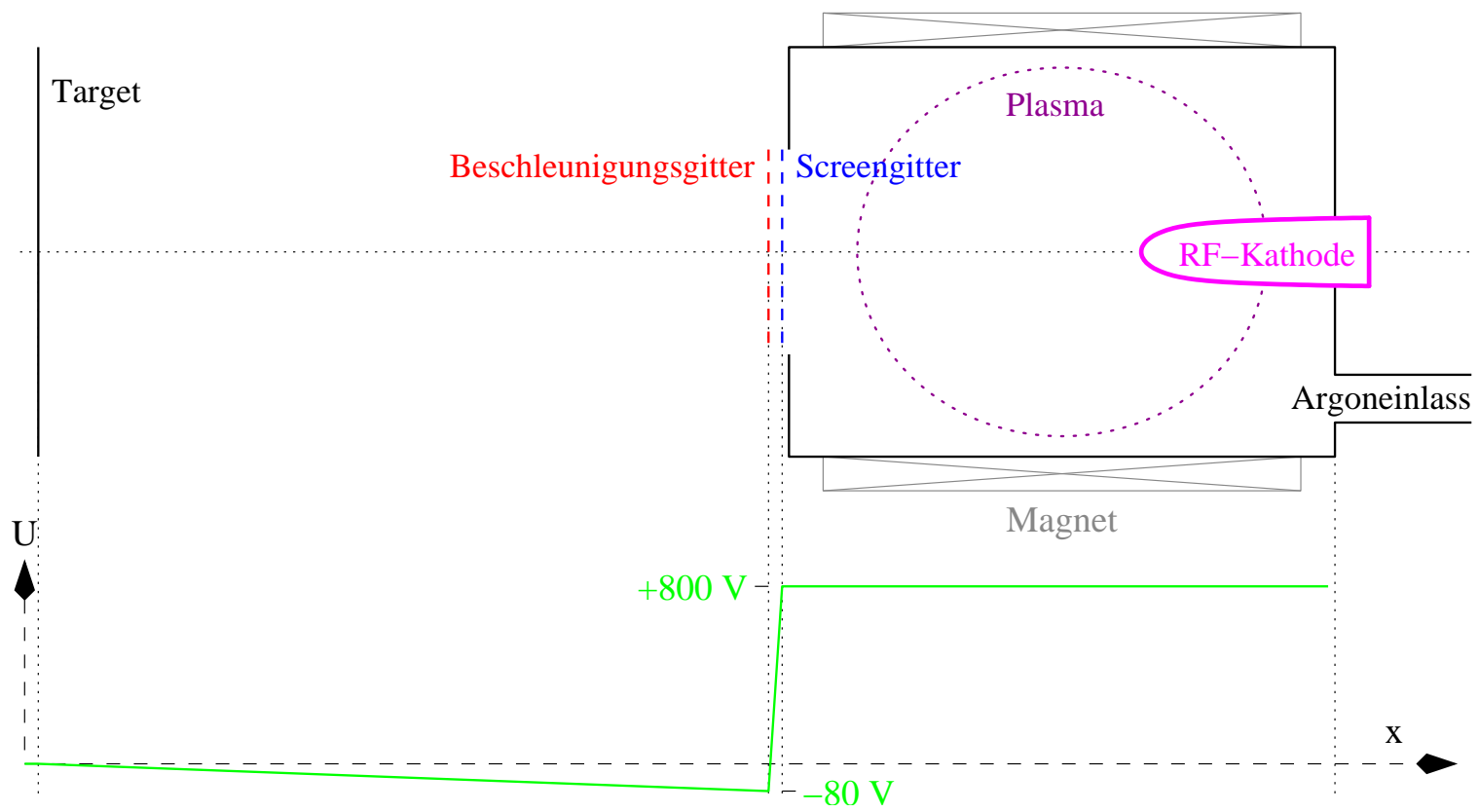

Abbildung 3.5: Schematischer Aufbau der Zweigitter Ar-Ionenquelle. Mit der RF-Kathode wird das Argon ionisiert und beschleunigt. Das Magnetfeld zwingt die Ionen auf Kreisbahnen, so entsteht ein Plasma. Durch die beiden identischen Gitter wird ein gerichteter Ar-Strahl erzeugt, der auf das Target trifft. Im unteren Teil ist der Verlauf der Spannung gegenüber dem Ort aufgetragen.

\subsection{Herstellung und Charakterisierung von Nb-Schichten}

Die Proben für diese Arbeit wurden mit Hilfe von HV-Sputterdeposition hergestellt, wobei mit einer Ar-Ionenquelle Argonionen zu einem Target hin beschleunigt werden. Bei der Argonionenquelle handelt es sich um eine Zweigitter RF-Quelle der Firma Plasma Consult. In Abb. 3.5 ist ein schematischer Querschnitt der Ionenquelle dargestellt. Durch Argoneinlass wird Argon mit der Reinheit 5.0 über einen Flussregler eingelassen. Während der Schichtherstellung wurde ein Fluss von $0.65 \mathrm{sccm}$ eingestellt, wodurch sich in der Kammer ein Druck von ca. $1 \cdot 10^{-4}$ mbar einstellt. Mit der Kathode wird eine Hochfrequenz von $13.6 \mathrm{MHz}$ mit einer Leistung von $76 \mathrm{~W}$ eingekoppelt, die die Argonatome ionisieren. Mit Hilfe des äußeren Magnetfeldes werden die erzeugten Elektronen und Ionen auf Kreisbahnen gezwungen und erzeugen durch Stöße neue Elektronen und Ionen im so entstehenden Plasma. Die beiden Gitter dienen dazu, einen Teil der Argonionen aus dem Plasma zu beschleunigen. Mit dem ersten Gitter (Screengitter) wird das Plasma auf ein Potential von $U_{\mathrm{scr}}=+800 \mathrm{~V}$ gehoben. Das zweite Gitter (Beschleunigungsgitter) liegt auf einem Potential von $U_{\text {acc }}=-80 \mathrm{~V}$. Die beiden identischen Gitter, die in einem Abstand von ca. $1 \mathrm{~mm}$ angeordnet sind, sorgen für einen gerichteten Ionenstrahl auf das Target. Vom wassergekühlten Target 


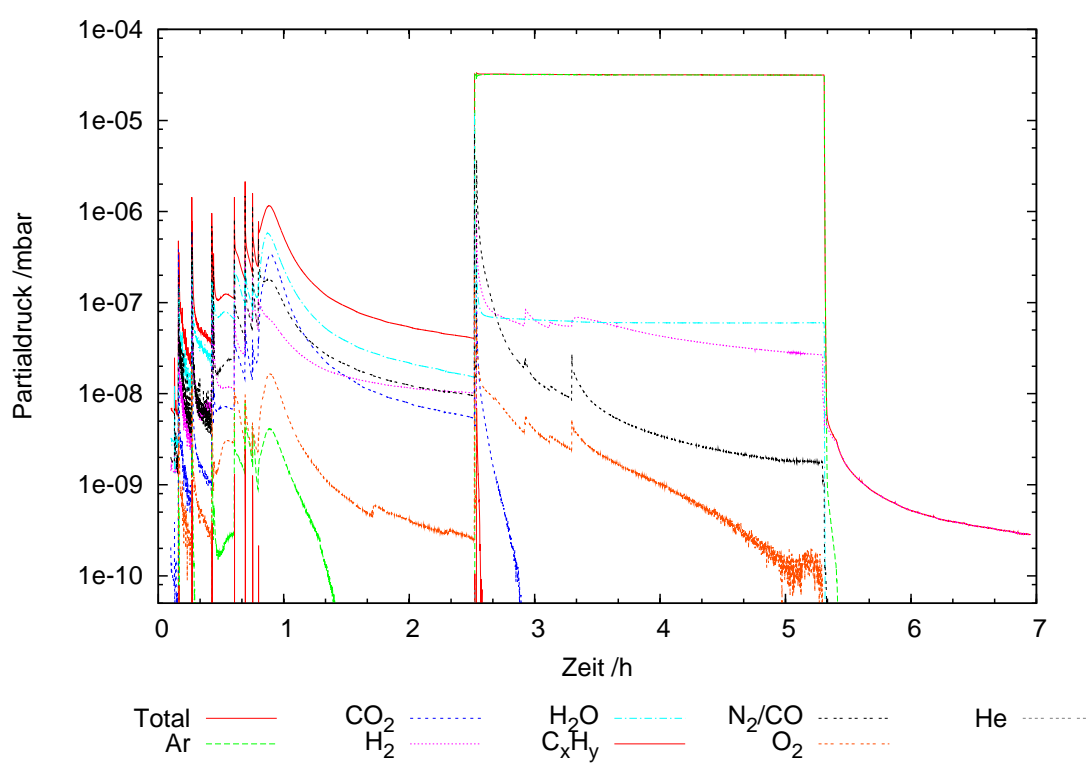

Abbildung 3.6: Restgasanalyse während der Schichtpräparation. Beim stufenweisen Erhitzen auf $800^{\circ} \mathrm{C}$ sind jeweils Peaks zu erkennen. Beim eigentlichen Sputtern (hoher Ar-Anteil im Restgas) erklären sich die Stufen durch die Leistungserhöhung der Quelle.

wird das Material auf das Substrat deponiert. Das Substrat wurde vor dem Sputtern auf eine Temperatur von $800{ }^{\circ} \mathrm{C}$ geheizt und während des Sputterns bei dieser gehalten. Vor Beginn der Schichtdeposition wurde das Substrat ca. 90 min bei dieser Temperatur gehalten, damit der Restgasdruck in der Kammer durch das ständige Abpumpen vor dem Depositionsbeginn verringert wird. In Abb. 3.6 ist eine Restgasanalyse während der Schichtherstellung dargestellt. Beim stufenweisen Erhitzen des Substrates sind diese Stufen auch im Kammerdruck zu sehen. Wenn nach dem Heizen der Probe das Ar eingelassen wird, ist zu Beginn ein Ansteigen aller Partialdrücke zu erkennen. Die Schichterzeugung wurde ca. 40 min nach Ar-Einlass begonnen, damit die Partialdrücke wieder abgesunken sind und die obere Targetschicht, die oxidiert bzw. verunreinigt ist, durch den Argonbeschuss gereinigt wird. Die Peaks bei den Kurven für $\mathrm{O}_{2}$ und $\mathrm{N}_{2} / \mathrm{CO}$ sind auf die Leistungserhöhung der Quelle zurückzuführen.

\subsubsection{Präparation epitaktischer Niobschichten}

Über das epitaktische Aufwachsen von Niob auf Saphir bei hohen Depositionstemperaturen wird in der Literatur mehrfach berichtet [WMTB01, ODD ${ }^{+}$98, YZSK95]. Es besteht eine feste dreidimensionale Beziehung zwischen den beiden Kristallgittern. Diese Orientierung ist in Abb. 3.7 dargestellt und wächst bei der Deposition bevorzugt. Wenn Niob auf (11̄̄0) Saphir deponiert wird, entsteht so eine (110)-texturierte Schicht. Diese Orientierung ist bei den hergestellten Schichten verwendet worden. Andere Texturen können erzeugt werden, wenn unterschiedlichen Substratoberflächen zum Einsatz kommen. Da es aus technischen Gründen schwer ist ein Substrat herzu- 

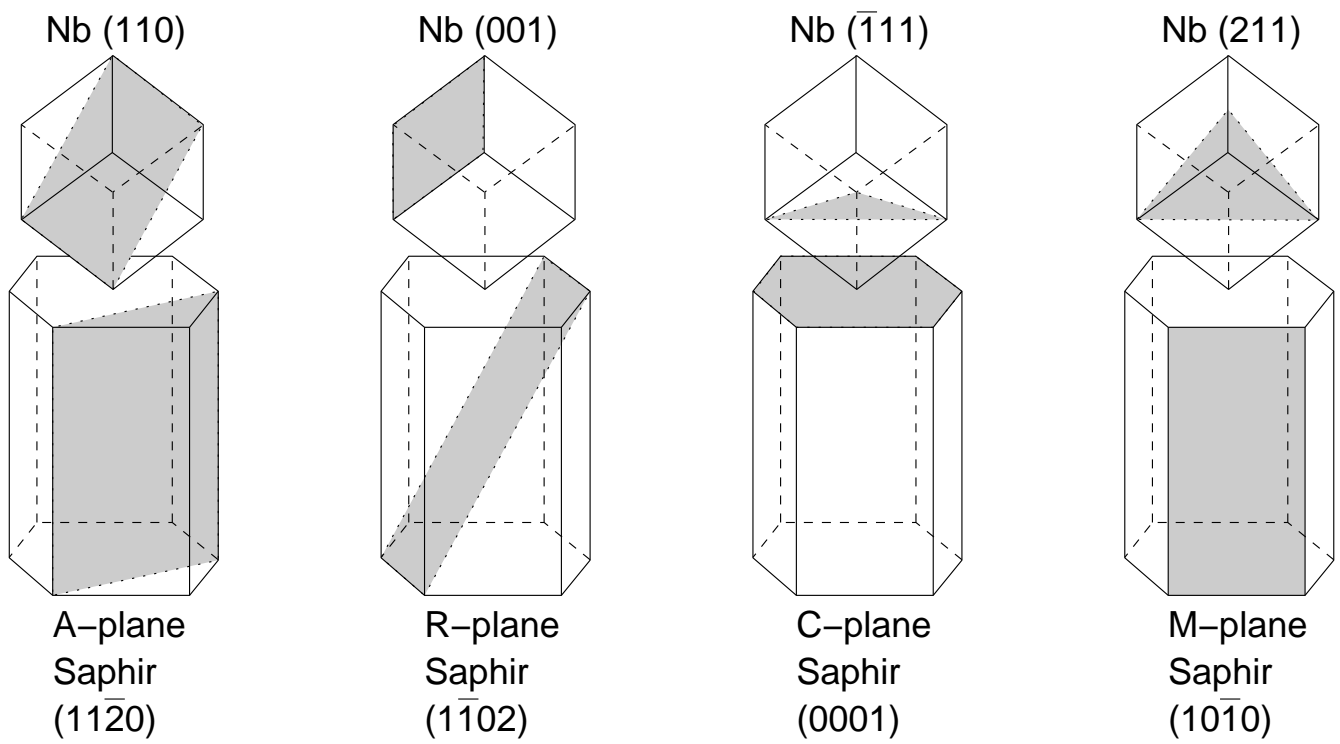

Abbildung 3.7: Orientierungsbeziehung von Niob auf Saphir. Zwischen den Gittern von Saphir und Niob besteht eine dreidimensionale Orientierungsbeziehung. Je nach gewählter Substratoberfläche wächst eine andere Kristallrichtung des Niobfilms [WMTB01]. Hier wurde A-plane Saphir für die Herstellung gewählt.

stellen, das die gewünschte Orientierung genau erfüllt, wird der Miscut $\vartheta$ eingeführt. Ein Miscut von $\vartheta<0.1^{\circ}$ bedeutet, dass die Oberflächenorientierung weniger als $0.1^{\circ}$ von der angegebenen Richtung abweicht.

Damit eine epitaktische Schicht entsteht, müssen die richtigen Herstellungsparameter gewählt werden. Die Depositionsrate der Atome ist so einzustellen, dass sich die Atome auf der Oberfläche bewegen können und die energetisch günstigste Position erreichen, bevor ein weiteres Atom auf die Oberfläche trifft und die Beweglichkeit behindert. Die Schicht soll im Lagenwachstum entstehen und das Inselwachstum sollte unterdrückt sein, damit sich der gewünschte Niobfilm bildet. Die Beweglichkeit der Atome auf der Oberfläche hängt stark von der Temperatur ab. Folglich sind die Sputterrate und die Temperatur die entscheidenden Faktoren für die Entstehung einer energetisch günstigen epitaktischen Beziehung zwischen Substrat und Film. Bei der Schichtherstellung betrug die Depositiontionsrate des Niobs ca. $0.6 \mathrm{~nm} / \mathrm{min}$. Die Deposition fand bei einer Substrattemperatur von $800^{\circ} \mathrm{C}$ statt.

\subsubsection{Charakterisierung der Schichten}

Um zu überprüfen, ob diese Orientierungsbeziehung bei den gewählten Herstellungsparametern erfüllt wird, wurden (110)-Texturmessungen durchgeführt. Das Ergebnis der Messung eines $100 \mathrm{~nm}$ dünnen Niobfilms ist in Abb. 3.8 dargestellt. Aufgetragen wurde die Röntgenintensität in Abhängigkeit von beiden Verkippungswinkeln. Es sind deutlich fünf (110)-Reflexe zu sehen, wie dies bei einer (110)-Epitaxie erwartet 


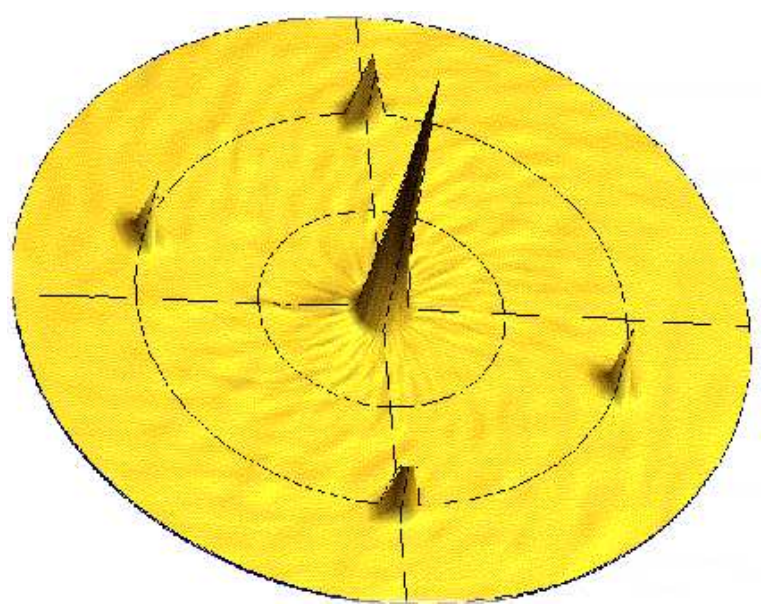

Abbildung 3.8: (110) Texturdarstellung eines $100 \mathrm{~nm}$ dünnen Nb-Films auf $(11 \overline{2} 0)$ Saphir $\left(800^{\circ} \mathrm{C}\right.$ Herstellungstemperatur). Aufgrund der Lage der fünf (110)-Reflexe handelt es sich um eine (110)-Epitaxie.

wird. Bei fehlender Orientierungsbeziehung zum Substrat wäre ein Ring zu erwarten. Der theoretisch erwartete Winkel von $60^{\circ}$ zwischen den Reflexen wird in der Messung verifiziert. Somit erhält man mit der eingesetzten Herstellungstemperatur und Sputterrate einen epitaktischen Niobfilm.

Eine $400 \times 400 \mathrm{~nm}^{2}$ große STM-Aufnahme eines $100 \mathrm{~nm}$ dünnen Nb-Films ist in Abb. $3.9 \mathrm{zu}$ sehen. Im oberen STM-Bild ist durch die Farbkodierung der Höhe deutlich der totale Höhenunterschied von $3 \mathrm{~nm}$ zu erkennen. Die gebildeten Terrassen haben einen Durchmesser von ca. $80 \mathrm{~nm}$. Der Höhenunterschied zwischen den einzelnen Terrassen beträgt $0.2 \mathrm{~nm}$. Das entspricht einer monoatomaren Stufe entlang der (110)-Richtung. Die Rauigkeit (RMS) auf den Terrassen beträgt $0.05 \mathrm{~nm}$. Im unteren Bild ist der Gradient in eine Richtung der Schicht abgebildet (Schattenmethode). Hier werden die Terrassenränder und die Enden dieser Ränder deutlich sichtbar. In der Abbildung rechts unten sind die Enden der Terrassenstufen durch rote Kreise markiert. Bei einem Umlauf entlang eines solchen Kreises wird genau eine monoatomare Stufe überschritten. Dies charakerisiert eine Schraubenversetzung. Somit sind die Enden der Terrassenränder Durchstoßpunkte von Versetzungslinien durch die Filmoberfläche.

Durch Auszählen dieser Terrassenenden erhält man die Anzahl von Versetzungen pro Fläche. Wenn angenommen wird, dass die Versetzungslinien senkrecht zur Substrat-Schicht-Grenzfläche sind, kommt man auf eine Versetzungsdichte der Schraubenversetzungen von

$$
7.1(6) \cdot 10^{10} \mathrm{~cm}^{-2}
$$

in der nicht beladenen Schicht. Diese schon durch die Herstellung vorhandene Versetzungsdichte wird im Folgenden intrinsische Versetzungsdichte genannt und ist von den Versetzungen, die durch die Veränderung während der Wasserstoffbeladung entstehen, zu unterscheiden.

Die gemessene Versetzungsdichte entspricht der in der Literatur für epitaktische Schichten angegebenen Größenordnung von $10^{10}-10^{11} \mathrm{~cm}^{-2}\left[\mathrm{KKM}^{+} 02, \mathrm{RPBS} 96\right.$, Pas63]. B. Karunagaran et.al. [KKM $\left.{ }^{+} 02\right]$ haben Röntgenanalyse betrieben, um die Versetzungsdichte zu bestimmen. E. A. Romanov et.al. [RPBS96] schreiben, 

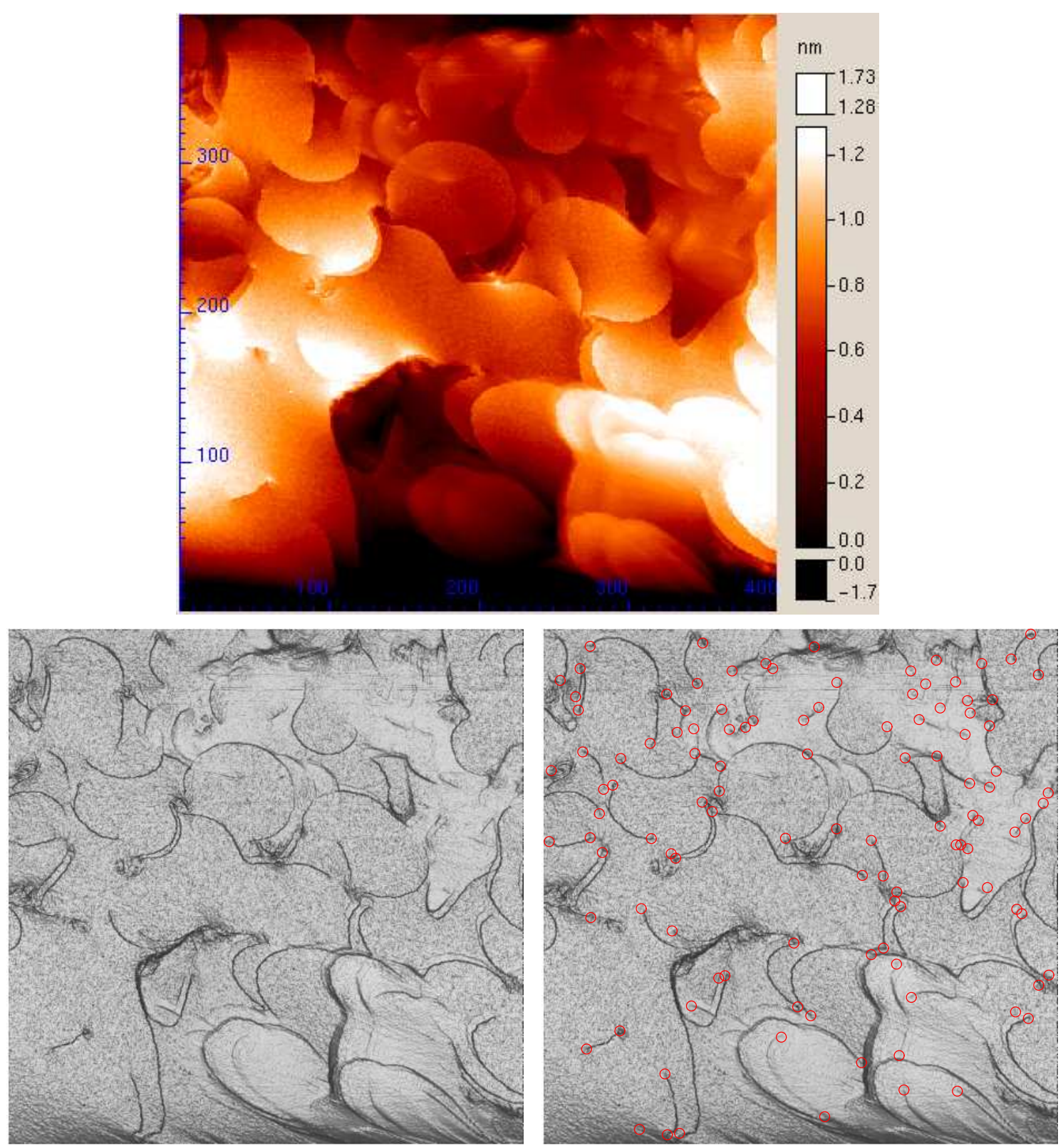

Abbildung 3.9: STM-Aufnahme eines $100 \mathrm{~nm}$ dünnen Nb-Films auf (11̄̄0) Saphir, bei $800^{\circ} \mathrm{C}$ hergestellt, die direkt nach der Präparation erstellt wurde. Im oberen STM-Bild ist die Höhe farbcodiert dargestellt und in den unteren Bildern ist die Ableitung der Höhe in eine Richtung (Schattenmethode) als Graustufen zu sehen. Es sind Terrassen mit monoatomaren Stufen zu sehen. Am Ende der Terrassen sind Durchstoßpunkte von Schraubenversetzungen, die unten rechts noch einmal durch Kreise hervorgehoben wurden. Mit der so bestimmten Anzahl der Versetzungen kann die Versetzungsdichte von $\rho=7.1(6) \cdot 10^{10} \mathrm{~cm}^{-2}$ bestimmt werden. Bildparameter: Größe $400 \times 400 \mathrm{~nm}^{2}, \mathrm{U}_{\text {gab }}=-$ $1 \mathrm{~V}, I=1 \mathrm{nA}$ 


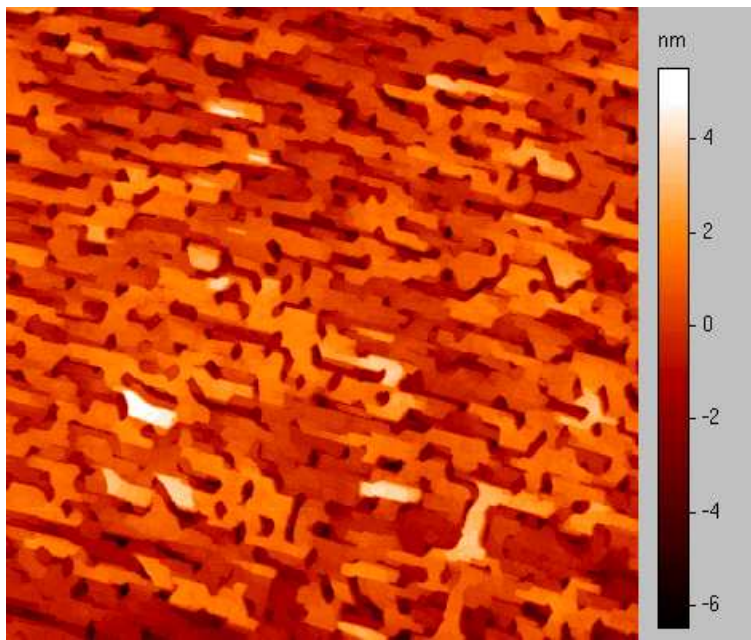

(a) $2.5 \times 2.5 \mu \mathrm{m}^{2}$

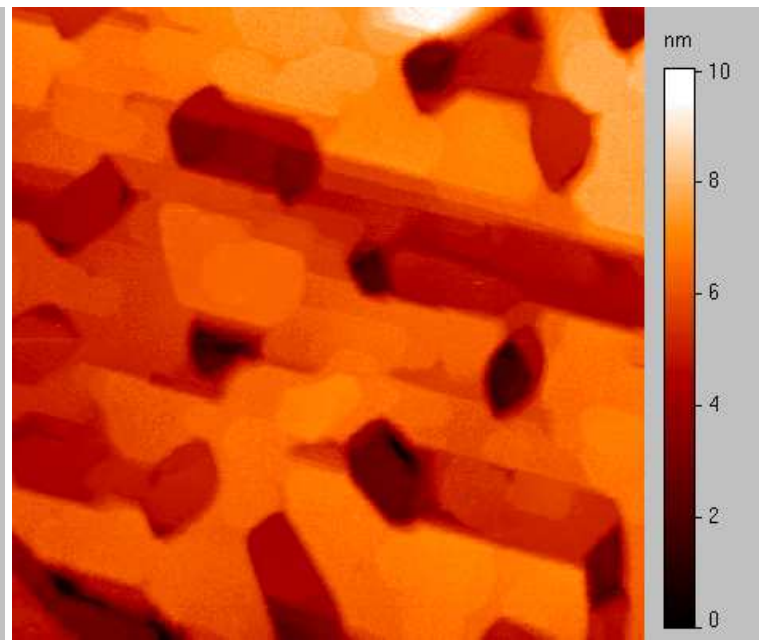

(b) $0.5 \times 0.5 \mu \mathrm{m}^{2}$

Abbildung 3.10: $20 \mathrm{~nm}$ asprepared Nb-Film. Die Oberfläche hat eine sehr offene Struktur. Im Übersichtsbild (a) tritt ein Höhenunterschied von ca. $11 \mathrm{~nm}$ auf. In der Detailaufnahme (b) sind sehr ebene Terrassen neben tiefen Tälern zu sehen. Bildparameter: $U_{\text {gab }}=-0.34 \mathrm{~V}, I=0.5 \mathrm{nA}$

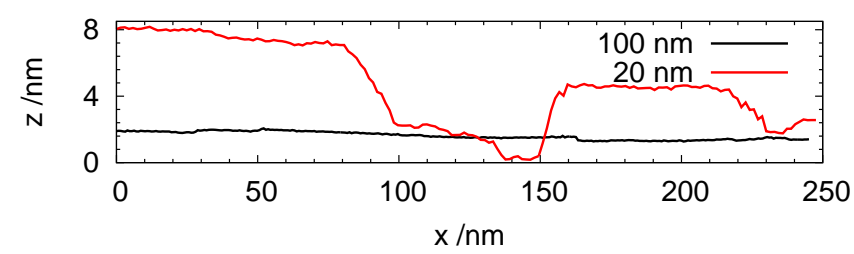

Abbildung 3.11: Höhenprofile von Schichten mit $20 \mathrm{~nm}$ und $100 \mathrm{~nm}$ Dicke. Mit steigender Filmdicke sinkt die Oberflächenunebenheit (RMS-Wert auf den Terrassen und Inselhöhe).

dass für Gitterfehlpassungen von ca. 2\% (was bei $\mathrm{Nb}$ auf $\mathrm{Al}_{2} \mathrm{O}_{3}$ der Fall ist) diese Größenordnung von Versetzungsdichten auftreten. Die Messungen von D. W. PASHLEY [Pas63] wurden mit einem Elektronenmikroskop durchgeführt.

Bei geringeren Filmdicken ist die Oberfläche wesentlich rauher. Ein solcher Film ist in Abb. 3.10 dargestellt. Bei diesem $20 \mathrm{~nm}$ dünnen Film, der auf einem Saphirsubstrat mit einem Miscut $\vartheta<0.1^{\circ}$ hergestellt wurde, beträgt der Höhenunterschied an der Oberfläche ca. $11 \mathrm{~nm}$, wie dies in dem $2.5 \times 2.5 \mu \mathrm{m}^{2}$ großen Ausschnitt in Teilbild (a) zu erkennen ist. Mit einer Schichtdicke von $20 \mathrm{~nm}$ ist hier eine sehr offene Struktur vorhanden. Die Inselbildung hat bei dieser Herstellung gegenüber der $100 \mathrm{~nm}$ dünnen Schicht zugenommen. In der Detailaufnahme in Teil (b) ist zu sehen, dass die gebildeten Terrassenoberflächen glatt sind. Die RMS-Rauigkeit auf einer solchen Terrasse beträgt $0.15 \mathrm{~nm}$. Die Terrassenränder bestehen zum Großteil aus Stufen von mehr als $3 \mathrm{~nm}$ Höhe. Monoatomare Stufen sind nur sehr wenige zu finden. Die Terrassengröße beträgt ca. $100 \mathrm{~nm}$ und ist somit von der gleichen Größe wie bei der $100 \mathrm{~nm}$ dünnen Schicht aus Abb. 3.9. Zum Vergleich der Unebenheiten beider Schichten sind in Abb. 3.11 Höhenprofile von der $100 \mathrm{~nm}$ Schicht sowie der $20 \mathrm{~nm}$ dünnen Schicht 


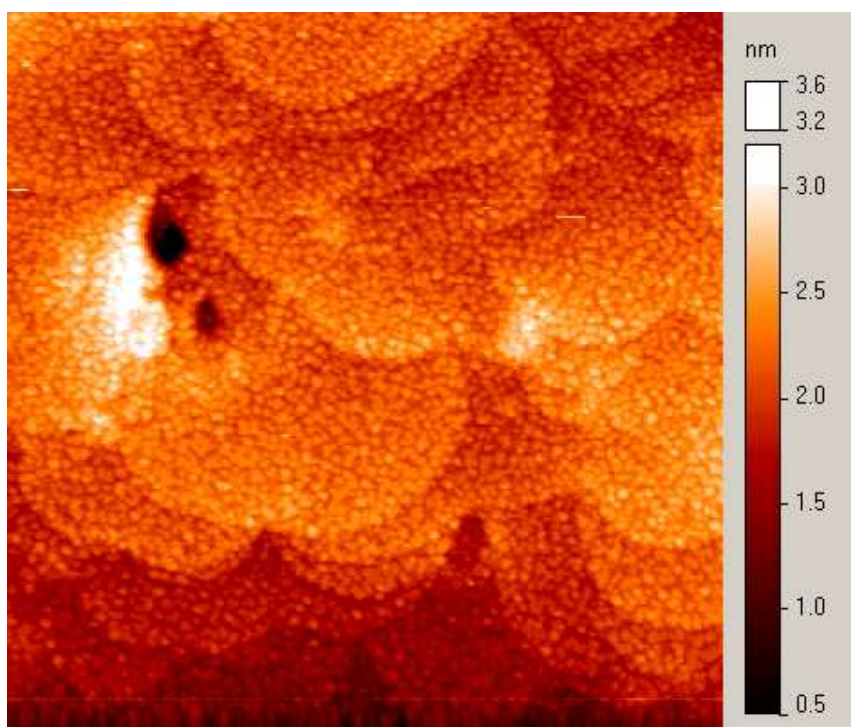

(a) $1.5 \mathrm{~nm}$ Pd, Größe $400 \times 400 \mathrm{~nm}^{2}$

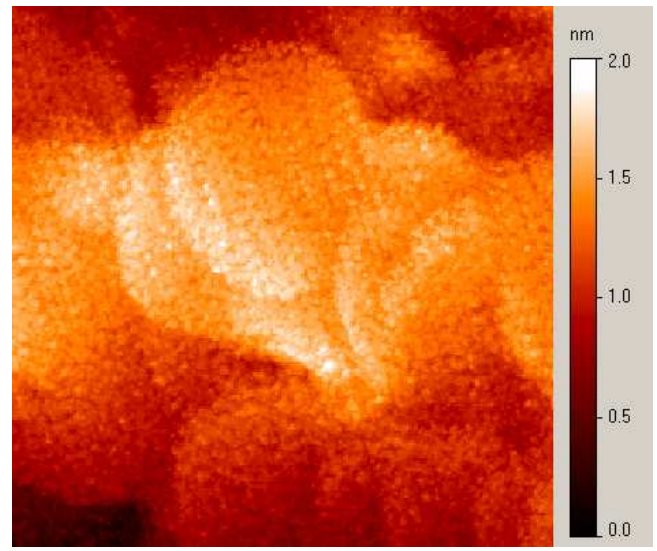

(b) $0.2 \mathrm{~nm} \mathrm{Pd}$, Größe $200 \times 200 \mathrm{~nm}^{2}$

Abbildung 3.12: Zwei verschiedene $100 \mathrm{~nm}$ dünne Niobfilme bedeckt mit Palladium. In (a) ist $1.5 \mathrm{~nm} \mathrm{Pd}$ deponiert worden. Es sind deutlich Pd-Inseln zu erkennen. Auf die Schicht in Teil (b) ist $0.2 \mathrm{~nm} \mathrm{Pd}$ aufgebracht worden. Bei dieser geringen Menge sind die Inseln nur schemenhaft zu sehen. Im Wesentlichen wird eine Erhöhung der Rauigkeit sichtbar. Beide aufgebrachten Mengen Palladium genügen um, den Wasserstoff an der Oberfläche dissoziieren zu lassen.

dargestellt. Es ist deutlich zu sehen, dass die Rauigkeit auf den Terrassen und die Stufenhöhe zwischen den Terrassen mit zunehmender Schichtdicke abnimmt. Wenn im Folgenden von Unebenheit gesprochen wird, bezieht sich dies auf die Inselhöhe, also den RMS-Wert von einem großen Gebiet, das mehrere Terrassen einschließt.

\subsubsection{Niobschichten für die Gasbeladung}

Da eine reine Nioboberfläche im Experiment bei Zimmertemperatur auch bei einem Wasserstoffdruck bis zu 1 mbar keinen Wasserstoff aufnimmt, wurde auf der Schicht noch geringfügig Palladium als Katalysator deponiert. Um die Durchmischung beider Metalle möglichst zu vermeiden, wurde diese Deposition erst nach dem Abkühlen auf Zimmertemperatur durchgeführt. STM-Aufnahmen von Niobschichten, die mit etwas Palladium bedeckt sind, sind in Abb. 3.12 dargestellt. Es sind zwei $100 \mathrm{~nm}$ dünne Filme zu sehen. In Abb. 3.12(a) ist auf der Schicht soviel Pd deponiert worden, dass die Menge einer Schichtdicke von $1.5 \mathrm{~nm}$ entspricht. Es ist deutlich zu erkennen, dass sich keine geschlossene Schicht ausbildet, sondern Inselwachstum stattfindet. Unter den Pd-Inseln sind die Terrassen der Niobschicht zu erkennen. Die Rauigkeit auf einer solchen Terrasse ist durch einen RMS-Wert von $0.12 \mathrm{~nm}$ auf einer Fläche von $100 \times 70 \mathrm{~nm}^{2}$ charakterisiert. Auf dem in Abb. 3.12(b) dargestellten Film wurde 


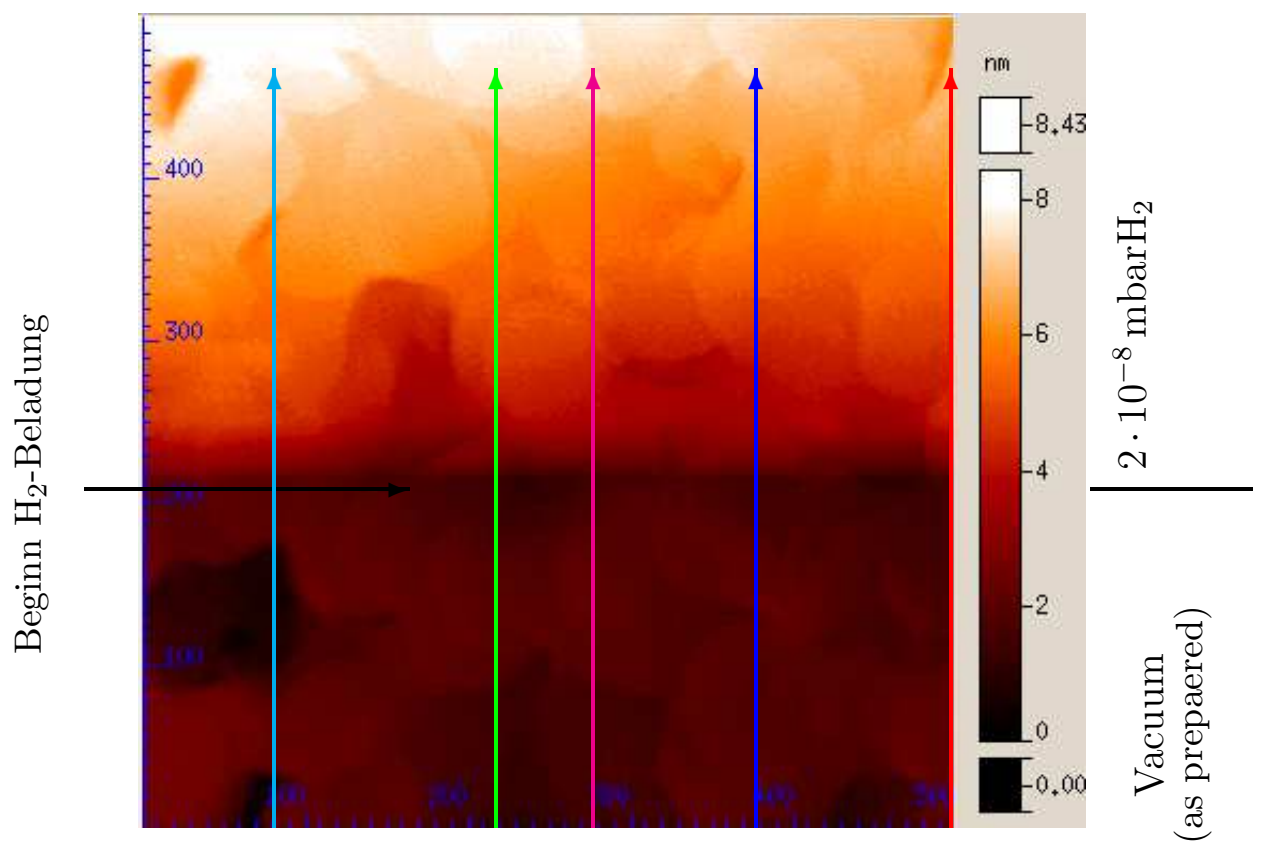

Abbildung 3.13: $100 \mathrm{~nm}$ dünner Niobfilm, bei dem während der Messung der Wasserstoffdruck variiert wurde. Zu Beginn lag der Druck bei $3 \cdot 10^{-10}$ mbar und wurde dann auf $2 \cdot 10^{-8}$ mbar $\mathrm{H}_{2}$ erhöht. Die Pfeile geben die Positionen der Höhenprofile an, die in Abb. 3.14 dargestellt sind. Einstellungen während der STM-Messungen: Größe $500 \times 500 \mathrm{~nm}^{2}, U_{\text {gab }}=-1 \mathrm{~V}, I=1 \mathrm{nA}$

$0.2 \mathrm{~nm}$ Palladium aufgebracht. Diese geringe Menge führt nicht zur Bildung einer geschlossenen Schicht, die Inseln sind nur schemenhaft zu erkennen. Der wesentliche Unterschied zu dem STM-Bild ohne Pd-Deckschicht (vgl. Abb. 3.9, S. 27) ist die größere Oberflächenrauigkeit auf den Terrassen (RMS-Wert von $0.07 \mathrm{~nm}$ in einem Gebiet von $75 \times 75 \mathrm{~nm}^{2}$ ). An diesen Pd-Inseln kann der Wasserstoff dissoziieren. Da die Löslichkeit des Wasserstoffs im Niob größer als im Palladium ist [Kes00], werden die Wasserstoffatome nach der Dissoziation an der Palladiumoberfläche in die Niobschicht diffundieren. Da beide aufgebrachten Mengen Palladium zur Dissoziation genügen, wurden für die STM-Untersuchungen nur die Menge von $0.2 \mathrm{~nm}$ Pd deponiert, um den Einfluss des Palladiums auf die beobachteten Veränderungen möglichst gering zu halten.

\subsubsection{Auftreten mechanischer Spannung in STM-Messungen}

Unter Wasserstoffaufnahme nimmt die mechanische Spannung im Schichtsubstratsystem erheblich zu. Spannungen bis zu -2.7 GPa wurden von U. LAUdAHN et.al. detektiert $\left[\mathrm{LPB}^{+}\right.$99]. Dies führt bei dünnen Substraten zur Substratkrümmung. Dieser Effekt wird dazu genutzt, die durch Wasserstoff erzeugten Spannungen auszumessen $\left[\mathrm{BvHL}^{+}\right.$98, $\mathrm{LFK}^{+}$99, $\mathrm{LPB}^{+}$99, $\mathrm{DPK}^{+}$03]. Im folgenden wird die Krümmung des Substrates während einer STM-Messung untersucht und berechnet. 


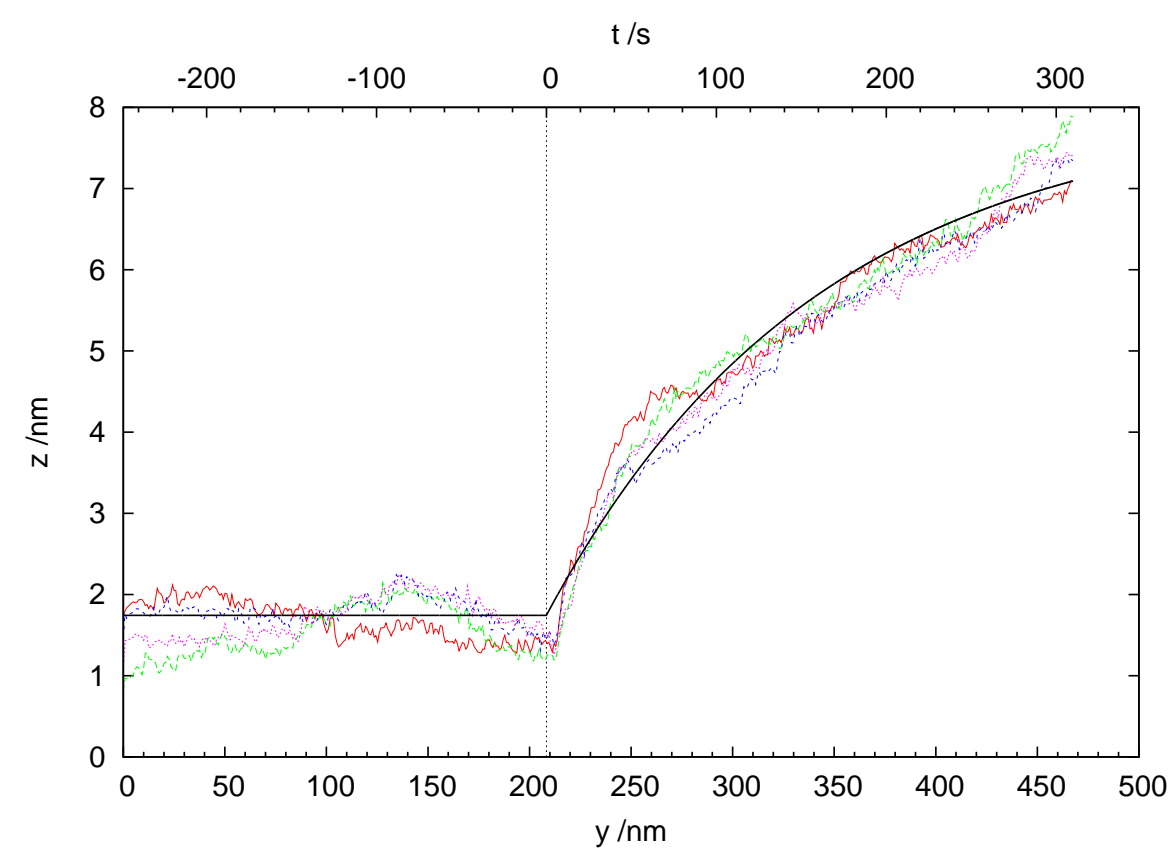

Abbildung 3.14: Höhenprofile der STM-Aufnahme von Abb. 3.13. Mit der Druckerhöhung bei $t=0 \mathrm{~s}$ (obere X-Achse) steigt die gemessene Höhe eines $100 \mathrm{~nm}$ dünnen Niobfilms mit dem exponentiellen Faktor $\left(6.2(2) \mathrm{nm} \cdot\left\{1-\mathrm{e}^{-m t}\right\}\right)$ an.

Der verwendete Niobfilm hat eine Dicke von $100 \mathrm{~nm}$ und wurde auf einem (1120) Saphirsubstrat von $0.43 \mathrm{~mm}$ Dicke und einem Miscut $\vartheta<0.1^{\circ}$ hergestellt. Ein STM-Bild des Films vor der Beladung ist in Abb. 3.12(b) gezeigt. In Abb. 3.13 wird das Verhalten bei einem Wasserstoffdruck von $2 \cdot 10^{-8}$ mbar gezeigt. Der Druck wurde während der STM-Messung in einem Schritt von $3 \cdot 10^{-10}$ mbar auf $2 \cdot 10^{-8} \mathrm{mbar}_{2}$ erhöht. Durch diese Druckveränderung dehnt sich die Schicht innerhalb der $\alpha$-Phase aus. Im unteren Teil der Abbildung betrug der umgebende Druck noch $3 \cdot 10^{-10}$ mbar. Deshalb kann die abgebildete Oberfläche noch gut durch eine Ebene beschrieben werden. Die geringen Abweichungen von dieser Ebene werden durch die Terrassen hervorgerufen, die sich bei der Schichtherstellung gebildet haben. Mit der Druckerhöhung, dessen Zeitpunkt in der Abbildung durch einen Pfeil markiert ist, steigt die Probenhöhe mit fortlaufender Zeilennummer (späterer Aufnahmezeitpunkt) kontinuierlich an. Dies wird durch die Höhenprofile in Abb. 3.14 deutlich sichtbar. Der theoretische zeitliche Verlauf berechnet sich aus Gl. (2.10) (s. Seite 8) und Gl. (2.29) (s. Seite 15) für $t>0$ durch die Gleichung

$$
\Delta z=\Delta z_{\text {total }}\left(1-\mathrm{e}^{-m t}\right) \quad .
$$

Die Zahlenwerte der Graphen in Abb. 3.14 sind

$$
\begin{aligned}
\Delta z_{\text {total }} & =6.2(2) \mathrm{nm} \\
m & =6.3(1) \cdot 10^{-3} \mathrm{~s}^{-1} .
\end{aligned}
$$




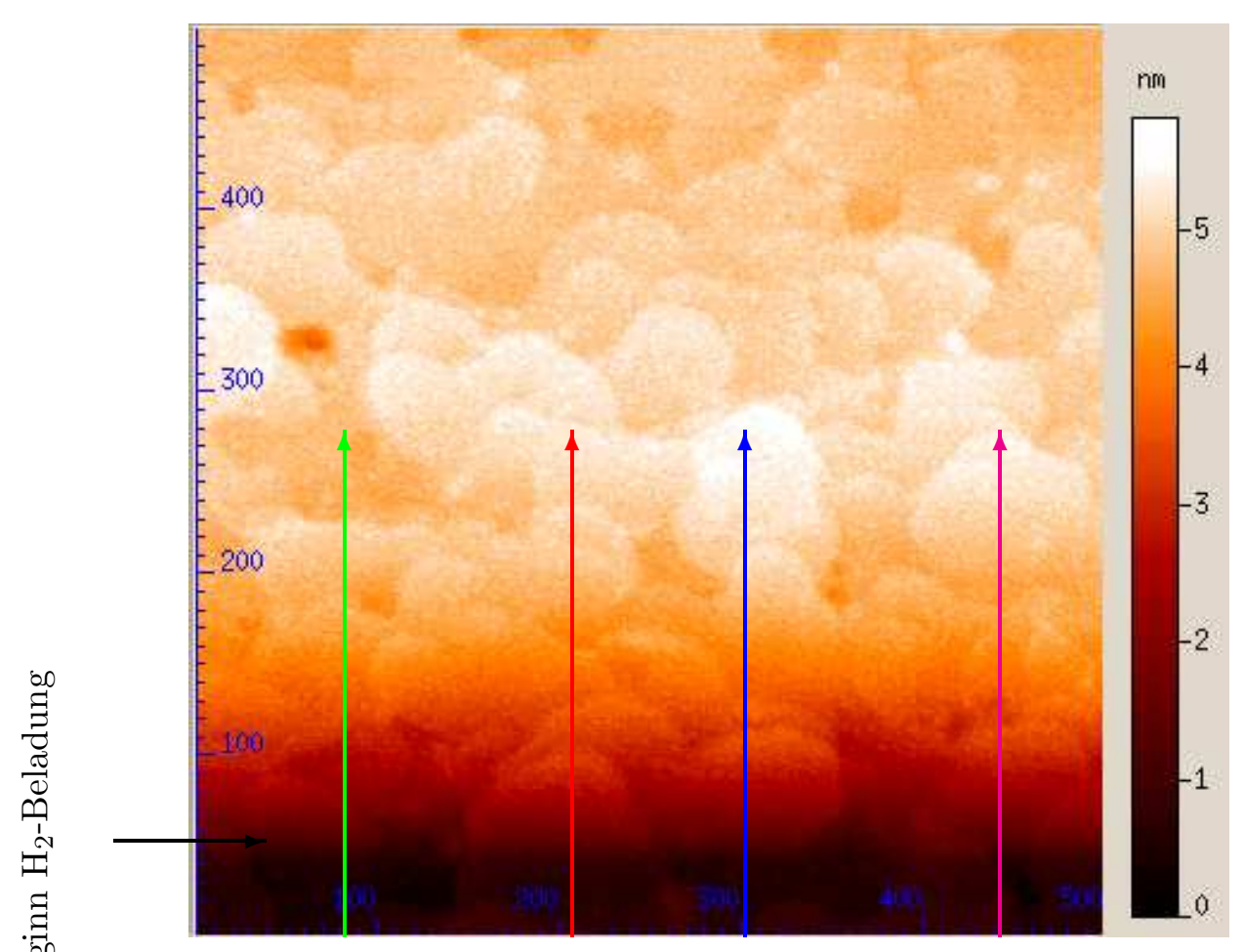

(a) STM-Bild, Größe $500 \times 500 \mathrm{~nm}^{2}, U_{\text {gab }}=0.8 \mathrm{~V}, I=1 \mathrm{nA}$

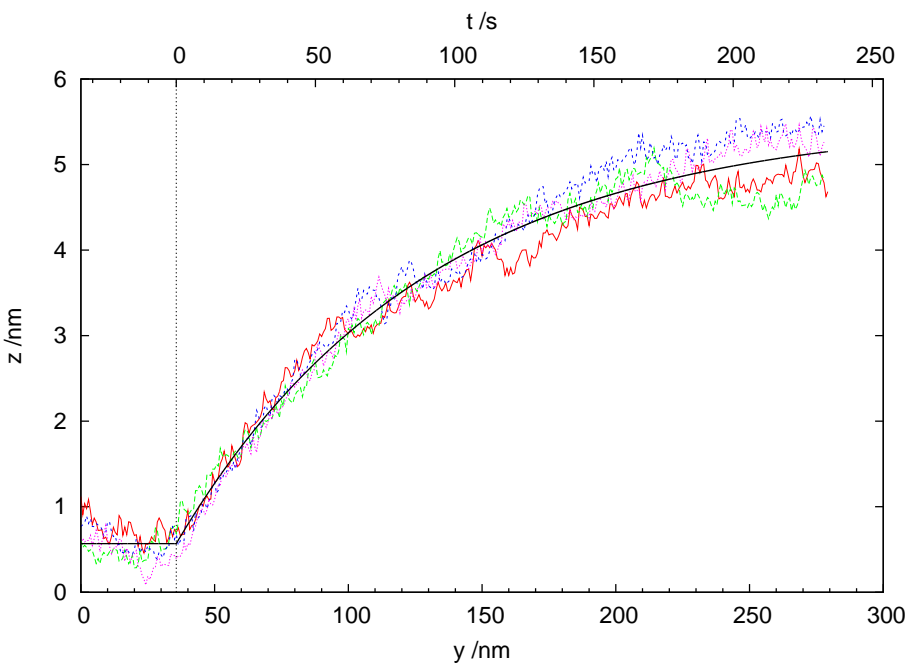

(b) Höhenprofile der eingezeichneten Pfeile

Abbildung 3.15: $50 \mathrm{~nm}$ dünner Niobfilm, beladen bei $1.6 \cdot 10^{-7} \mathrm{mbar} \mathrm{H}_{2}$. Die Höhe steigt bei der Wasserstoffzugabe mit dem Faktor $\left(4.9(2) \mathrm{nm} \cdot\left\{1-\mathrm{e}^{-m t}\right\}\right)$ an. 


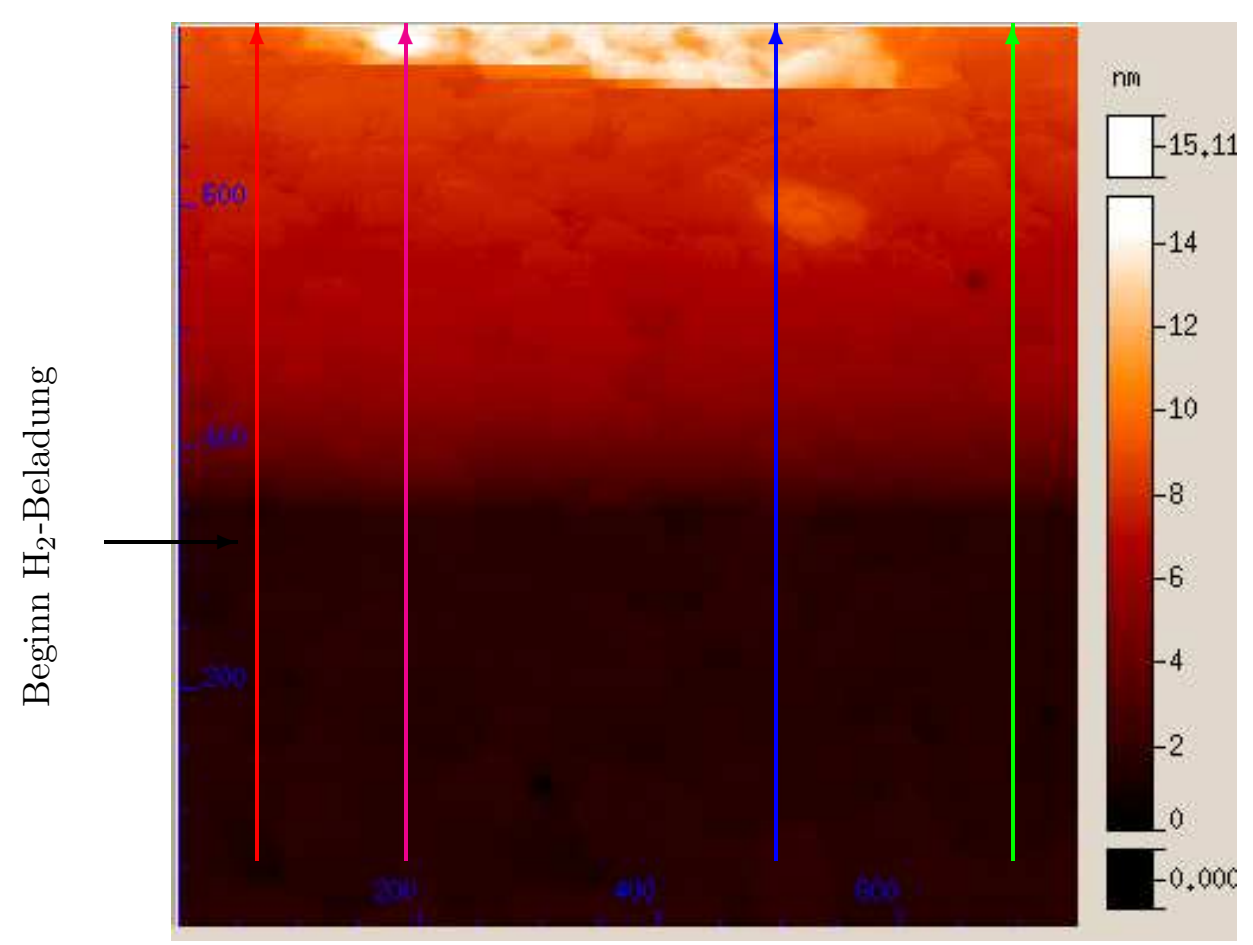

(a) STM-Bild, Größe $750 \times 750 \mathrm{~nm}^{2}, U_{\text {gab }}=0.8 \mathrm{~V}, I=1 \mathrm{nA}$

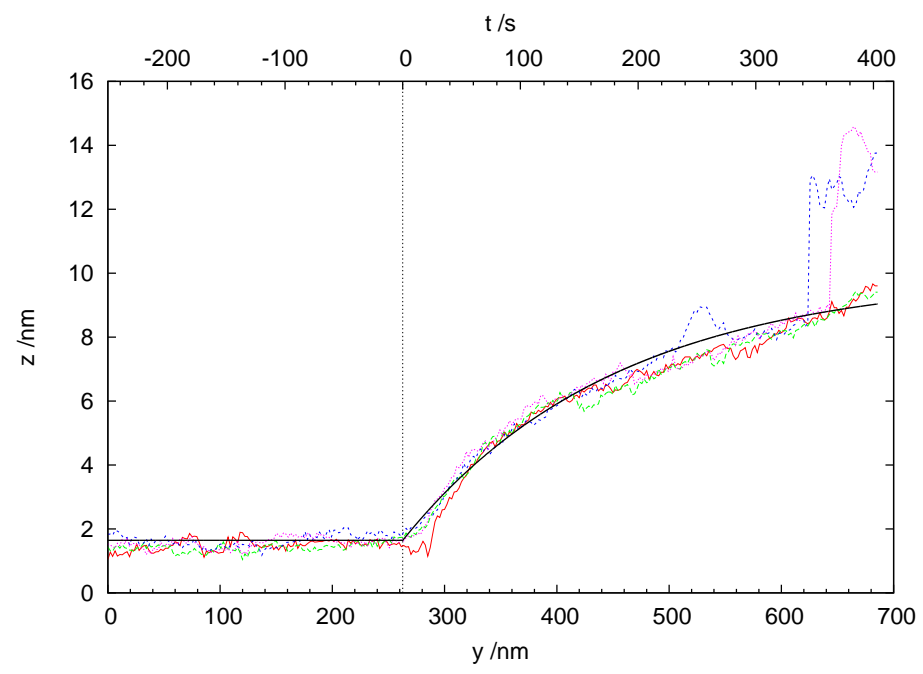

(b) Höhenprofile der eingezeichneten Pfeile

Abbildung 3.16: $50 \mathrm{~nm}$ dünner Niobfilm, beladen bei $1.4 \cdot 10^{-6} \mathrm{mbar}_{2}$. Es kommt bei der Wasserstoffzugabe zu einem Höhenanstieg mit dem Faktor $\left(8.3(2) \mathrm{nm} \cdot\left\{1-\mathrm{e}^{-m t}\right\}\right)$. Auf die Sprünge in den beiden Höhenprofilen wird in Abschnitt 4.2.1 eingegangen. 


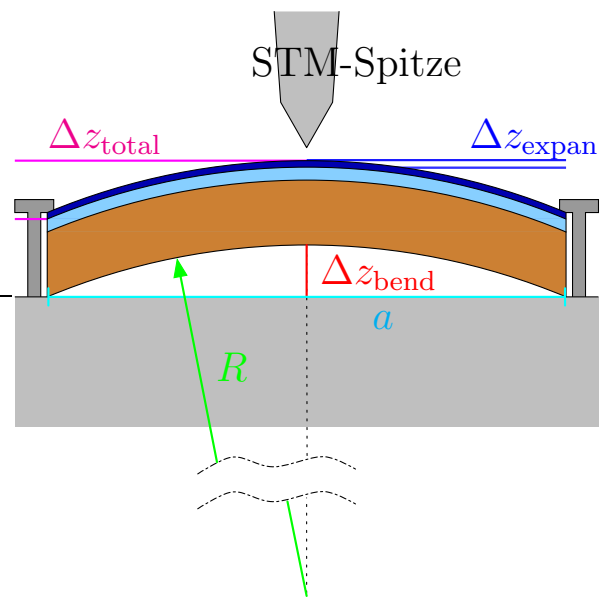

Abbildung 3.17: Krümmung des Substrats durch Spannungen im beladenen Nb-Film. Durch die Expansion des Films biegt sich das Substrat in der Mitte von der Substratunterlage weg.

Dieses Verhalten wurde auch bei einem $50 \mathrm{~nm}$ Nb-Film beobachtet, der auf einem $(11 \overline{2} 0)$ Saphirsubstrat (Miscut $\vartheta<0.1^{\circ}$ ) hergestellt wurde. Im ersten Beladungsschritt wurde der Druck von $4.2 \cdot 10^{-10}$ mbar auf $1.6 \cdot 10^{-7}$ mbar $\mathrm{H}_{2}$ erhöht (vgl. Abb. 3.15(a)). Dabei dehnte sich der Film entsprechend Gl. (3.4) um $\Delta z=4.9(2) \mathrm{nm}$ aus (vgl. Abb. 3.15(b)). Bei der folgenden Druckerhöhung auf $1.4 \cdot 10^{-6} \mathrm{mbar}_{2}$ (vgl. Abb. 3.16), betrug die Höhenänderung $\Delta z=8.3(2) \mathrm{nm}$. In Tabelle 3.1 sind die gemessenen Höhenzunahmen bei unterschiedlicher Schichtdicke und unterschiedlichem Druck zusammengestellt. Die theoretisch zu erwartende Schichtdickenzunahme aufgrund der Gitterdehnung $\Delta z_{\text {expan }}$ ist auch angegeben, diese bewirkt in allen drei Fällen weniger als $5 \%$ des Effektes. Die Konzentration in der Schicht wurde mit Gl. (2.37) (s. S. 16) aus dem eingestellten Wasserstoffdruck bestimmt. Dabei ist angenommen worden, dass sich bei einem Druck von $1.4 \cdot 10^{-6}$ mbar eine Konzentration von $10 \%$ einstellt, da bei diesem Druck die Hydridphase gebildet wurde. Die Werte sind im Wesentlichen durch die Substratkrümmung zu erklären, da eine Ausdehnung der Schicht mechanische Spannungen erzeugt. Durch die Spannungen wird die Probe in der Mitte von der Auflage entfernt, wie es in Abb. 3.17 dargestellt ist. Mit den Werten der gemessenen Krümmung $\Delta z_{\text {bend }}$ und der Probenlänge $a$ kann der Krümmungsradius bestimmt werden. Unter der Annahme, dass die STM-Spitze in der Probenmitte positioniert ist, besteht folgender Zusammenhang:

$$
R=\frac{\Delta z_{\text {bend }}}{2}+\frac{a^{2}}{8 \cdot \Delta z_{\text {bend }}}
$$

Falls $\Delta z_{\text {bend }}$ nicht genau in der Probenmitte gemessen wird, ist die Abweichung durch Gl. (3.5) aufgrund der zu einer Kugeloberfläche gebogenen Probe nur sehr gering. Bei bekanntem Krümmungsradius $R$ kann aus der STONEYschen Gleichung die mechanische Spannung berechnet werden [Sto09, Ohr02]

$$
\langle\sigma\rangle=-\frac{1}{6}\left[\frac{E}{1-\nu}\right] \frac{s^{2}}{h_{0}}\left(\frac{1}{R}\right)
$$


Tabelle 3.1: Berechnung der induzierten Spannung aus der Substratkrümmung. Die Konzentration $c_{\mathrm{H}}$ wurde mit Gl. (2.37) (s. S. 16) mit $S=84.5$ bestimmt, um mit dieser über Gl. (2.10) (s. S. 8) die Ausdehnung der Niobschicht $\Delta z_{\text {expan }}$ zu berechnen. Mit den Gleichungen (3.5) und (3.6) sind der Krümmungsradius $R$ und die Spannung $\langle\sigma\rangle$ ermittelt worden.

\begin{tabular}{|c|c|c|c|c|c|c|c|c|c|}
\hline $\begin{array}{c}h_{0} \\
{[\mathrm{~nm}]}\end{array}$ & $\begin{array}{c}p_{\text {start }} \\
{[\mathrm{mbar}]}\end{array}$ & $\begin{array}{c}p_{\text {end }} \\
{[\mathrm{mbar}]}\end{array}$ & $\begin{array}{c}c_{\mathrm{H}} \\
{[\%]}\end{array}$ & $\begin{array}{c}\Delta z_{\text {expan }} \\
{[\mathrm{nm}]}\end{array}$ & $\begin{array}{c}\Delta z_{\text {total }} \\
{[\mathrm{nm}]}\end{array}$ & $\begin{array}{c}\Delta z_{\text {bend }} \\
{[\mathrm{nm}]}\end{array}$ & $\begin{array}{c}R \\
{[\mathrm{~km}]}\end{array}$ & $\begin{array}{c}\langle\sigma\rangle \\
{[\mathrm{MPa}]}\end{array}$ & $\begin{array}{c}\langle\sigma\rangle / c_{\mathrm{H}} \\
{[\mathrm{GPa}]}\end{array}$ \\
\hline 100 & $3 \cdot 10^{-10}$ & $2.0 \cdot 10^{-8}$ & 1.2 & 0.16 & $6.2(2)$ & $6.1(2)$ & $2.05(7)$ & $-82(3)$ & $-6.88(22)$ \\
50 & $4.2 \cdot 10^{-10}$ & $1.6 \cdot 10^{-7}$ & 3.4 & 0.23 & $4.9(2)$ & $4.7(2)$ & $2.64(10)$ & $-128(5)$ & $-3.77(15)$ \\
50 & $1.6 \cdot 10^{-7}$ & $1.4 \cdot 10^{-6}$ & 10 & 0.68 & $13.2(3)^{*}$ & $12.5(3)$ & $0.99(2)$ & $-339(8)$ & $-3.39(8)$ \\
\hline
\end{tabular}

Hierbei gibt $E / 1-\nu=548.1 \mathrm{GPa}$ [HM84] die elastischen Eigenschaften des Saphirsubstrates wieder, $s=0.43 \mathrm{~mm}$ ist die Substratdicke und $h_{0}$ ist die Schichtdicke. Die Gleichung ist für rechteckige Proben mit einem Achsenverhältnis von mehr als 2:1 gültig. Für andere Achsenverhältnisse treten nach N. SCHWARzER und F. RICHTER Abweichungen von weniger als $4 \%$ auf [SR04].

Die gemessene Ausdehnung ist die Summe aus der Gitterdehnung $z_{\text {expan }}$ und der Substratkrümmung $z_{\text {bend }}\left(\Delta z_{\text {total }}=\Delta z_{\text {bend }}+\Delta z_{\text {expan }}\right)$. In Tabelle 3.1 ist die durch Gitterausdehnung und Substratkrümmung bewirkte Höhenänderung zusammengestellt. Mit dem aus Gl. (3.5) berechnetem Krümmungsradius $R$ wurde dann die in der Schicht vorhandene Spannung $\langle\sigma\rangle$ berechnet. Die Spannung pro Wasserstoffkonzentration $\langle\sigma\rangle / c_{\mathrm{H}}$ ist geringer als der theoretische Maximalwert von $-11.6 \mathrm{GPa}$ bzw. -7.6 GPa (s. Gl. (2.26) und Gl. (2.27), S 13). Dies ist damit zu erklären, dass nicht, wie in Gl. (3.6) vorausgesetzt, ein Ende der Probe frei ist. Weiterhin ist es möglich, dass die Spitze nicht ideal in der Substratmitte gemessen hat. Somit kann durch die Substratkrümmung die Höhenänderung erklärt werden. Um den Effekt der Substratkrümmung zu verringern, wurde die Substratdicke auf $1 \mathrm{~mm}$ erhöht. Bei dieser Dicke kann der Effekt vernachlässigt werden.

\subsection{Finite-Elemente-Methode}

Beim komplizierten Zusammenhang zur Berechnung von Hydridausscheidungen, die sich stärker als die umgebene $\alpha$-Phase ausdehnen, ist eine geschlossene Lösung nicht möglich. Aus diesem Grund wurde die Finite-Elemente-Methode verwendet, die Dehnungen und die Spannungen zu errechnen, die entstehen, wenn sich Teilbereiche einer Schicht ausdehnen. Dies ist im Zweiphasengebiet der Fall, wo Gebiete von $\alpha$-Phase und Hydrid mit verschiedener Gitterdehnung nebeneinader vorliegen. Für diese Berechnung ist das Programm COMSOL Multiphysics verwendet worden [COM].

Bei der Finite-Elemente-Methode wird ein kontinuierliches Problem in endlich viele kleine Elemente unterteilt. Auf diesen Elementen werden Ansatzfunktionen definiert,

\footnotetext{
*Summe aus den Werten beim Druck von $1.6 \cdot 10^{-7}$ mbar und $2.0 \cdot 10^{-6}$ mbar, da der Druck kontinuierlich erhöht wurde $\left(\Delta z_{1}=4.9(2) \mathrm{nm}, \Delta z_{2}=8.3(2) \mathrm{nm}, \Delta z_{\text {gesamt }}=13.2(3) \mathrm{nm}\right)$.
} 
aus denen über partielle Differentialgleichungen und den Randbedingungen ein großes Gleichungssystem bestimmt wird. Mit der Lösung dieses Gleichungssystems werden die gesuchten Resultate (Ausdehnung an der Oberfläche und gespeicherte elastische Energie) abgeleitet.

Für die Berechnung wird die Dehnung $\epsilon$ jeden Elements in einen elastischen Anteil $\left(\epsilon_{\text {elast. }}\right)$ und eine von außen $\left(\epsilon_{0}\right)$ vorgegebene Dehnung unterteilt:

$$
\epsilon=\epsilon_{\text {elast. }}+\epsilon_{0}
$$

Daraus ergibt sich für die auftretende Spannung $\sigma$ :

$$
\sigma=C_{i j} \cdot \epsilon_{\text {elast. }}+\sigma_{0}=C_{i j} \cdot\left(\epsilon-\epsilon_{0}\right)+\sigma_{0}
$$

wobei $C_{i j}$ die $6 \times 6$ Matrix der Elastitätsmodule in VoigTscher-Notation (s. S. 10) ist und $\sigma_{0}$ die von außen vorgegebene Anfangsspannung, die hier nicht berücksichtigt wird $\left(\sigma_{0}=0\right)$. Die Spannung $\sigma$ und die Dehnung $\epsilon$ sind Vektoren mit sechs Komponenten:

$$
\sigma=\left(\sigma_{x}, \sigma_{y}, \sigma_{z}, \tau_{x y}, \tau_{y z}, \tau_{x z}\right)^{\mathrm{t}} \quad \epsilon=\left(\epsilon_{x}, \epsilon_{y}, \epsilon_{z}, 2 \epsilon_{x y}, 2 \epsilon_{y z}, 2 \epsilon_{x z}\right)^{\mathrm{t}} \quad,
$$

die sich aus den Spannungen $\sigma_{i}$, Scherspannungen $\tau_{i j}$ und Dehnungen $\epsilon_{i j}$ zusammensetzen. Aus der Spannung $\sigma$ berechnen sich die Volumenkräfte $F$ :

$$
\vec{F}=-\nabla \cdot \sigma
$$

Aus diesen Gleichungen wird dann die im System gespeicherte totale Energie $W$ berechnet:

$$
\begin{aligned}
W= & \int_{V}\left\{-\frac{1}{2}\left(\epsilon_{x} \sigma_{x}+\epsilon_{y} \sigma_{y}+\epsilon_{z} \sigma_{z}+2 \epsilon_{x y} \tau_{x y}+2 \epsilon_{y z} \tau_{y z}+2 \epsilon_{x z} \tau_{x z}\right)+\vec{u} \cdot \overrightarrow{F_{V}}\right\} \mathrm{d} v \\
& +\int_{S}\left\{\vec{u} \cdot \vec{F}_{S}\right\} \mathrm{d} s+\int_{L}\left\{\vec{u} \cdot \overrightarrow{F_{L}}\right\} \mathrm{d} l+\sum_{P}\left\{\vec{u} \cdot \overrightarrow{F_{P}}\right\} .
\end{aligned}
$$

Dabei gibt $\vec{u}$ die Verschiebung an. Es beziehen sich $V$ auf Volumen, $S$ auf Oberflächen, $L$ auf Linien und $P$ auf Punkte, bzw. auf Kräfte, die in den jeweiligen Elementen definiert sind. Bei der hier durchgeführten Berechnung wurden keine äußeren Kräfte definiert. Aus der Energie- und Massenerhaltung folgt bei inkompressiblen Materialien wie bei Festkörpern:

$$
\begin{aligned}
\frac{\mathrm{d} V}{\mathrm{~d} V_{0}}=\frac{\rho_{0}}{\rho}=\operatorname{det}\left(\frac{\partial \vec{u}}{\partial \vec{x}}\right) & =1 \\
\delta W & =0 .
\end{aligned}
$$

Dieses sind die Gleichungen, die als Grundlage für die Finite-Element-Methode dienen. 


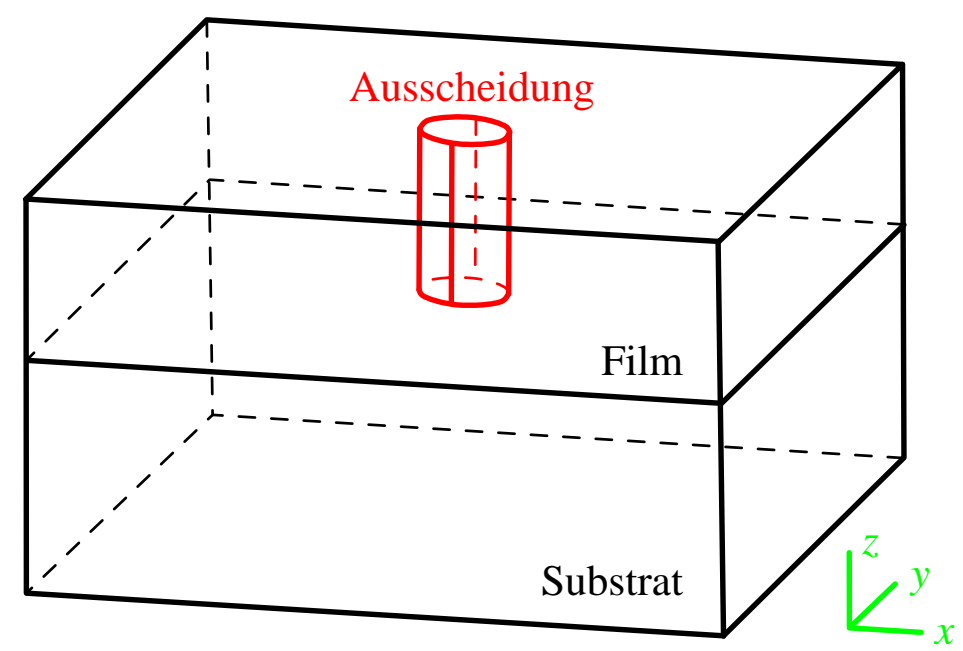

Abbildung 3.18: Berechnetes Gebiet der Finite-Elemente-Methode bei der Schichtgeometrie. Simuliert wurden unterschiedliche Ausscheidungsgeometrien in einem Nb-Film, der sich auf einem starren Saphirsubstrat befindet. Dargestellt ist ein Zylinder, der in $z$-Richtung durch die komplette Schicht reicht. Die Berechnung wurde mit einem starren Substrat durchgeführt. Eine Ausdehnung des Films über die Substratgrenzen in $x$ - und $y$-Richtung wurde nicht erlaubt.

\subsubsection{Ausscheidung in einer dünnen Schicht}

Die Finite-Element-Methode wurde auf unterschiedliche Ausscheidungsformen (Zylinder, Kugel und Ellipsoid) in einer dünnen Schicht angewendet. Das berechnete System ist in Abb. 3.18 dargestellt. Auf einem starren Saphirsubstrat befindet sich eine Niobschicht. Die Randbedingungen sind so gewählt, dass für das gesamte Substrat keine Ausdehnung und Krümmung erlaubt wurde. Beim Niobfilm ist eine seitliche Ausdehnung über den Rand des Substrates in $x$ - und $y$-Richtung nicht erlaubt. Damit wird berücksichtigt, dass die Berechnung nicht die komplette Schichtgröße simuliert. In diesem Film wurde in die Mitte eine Ausscheidung mit unterschiedlichen Geometrien gesetzt, die sich durch Wasserstoffbeladung ausdehnt. Folgende Geometrien wurden ausgewählt: ein Zylinder, der in z-Richtung durch die komplette Schicht reicht, eine Kugel und ein Ellipsoid mit dem Achsenverhältnis von $a_{x}: a_{y}: a_{z}=2: 2: 1$, die an unterschiedlichen Höhen positioniert sind. Bei den einzelnen Berechnungen sind die Filmdicke, die Größe und die Position der Ausscheidung variiert worden. Bei den Ausscheidungen wurde eine Dehnung von $2.9 \%$ in alle drei Raumrichtungen vorgegeben $^{\dagger}$ und anschließend die daraus resultierenden Spannungen und Dehnungen des Films berechnet. Zwischen Film und Ausscheidung wurde kein Abgleiten erlaubt, so dass die hier durchgeführten Berechnungen kohärente Ausscheidungen beschreiben. Bei der Wahl der Größe des berechneten Gebietes in $x$ - und $y$-Richtung wurde darauf

\footnotetext{
${ }^{\dagger}$ Es wurde die Dehnung gewählt, die für eine massive Niobprobe nach Gl. 2.1 und Gl. 2.3 (S. 5) bei einer Breite der Mischungslücke von $\Delta c=0.5$ erwartet wird.
} 


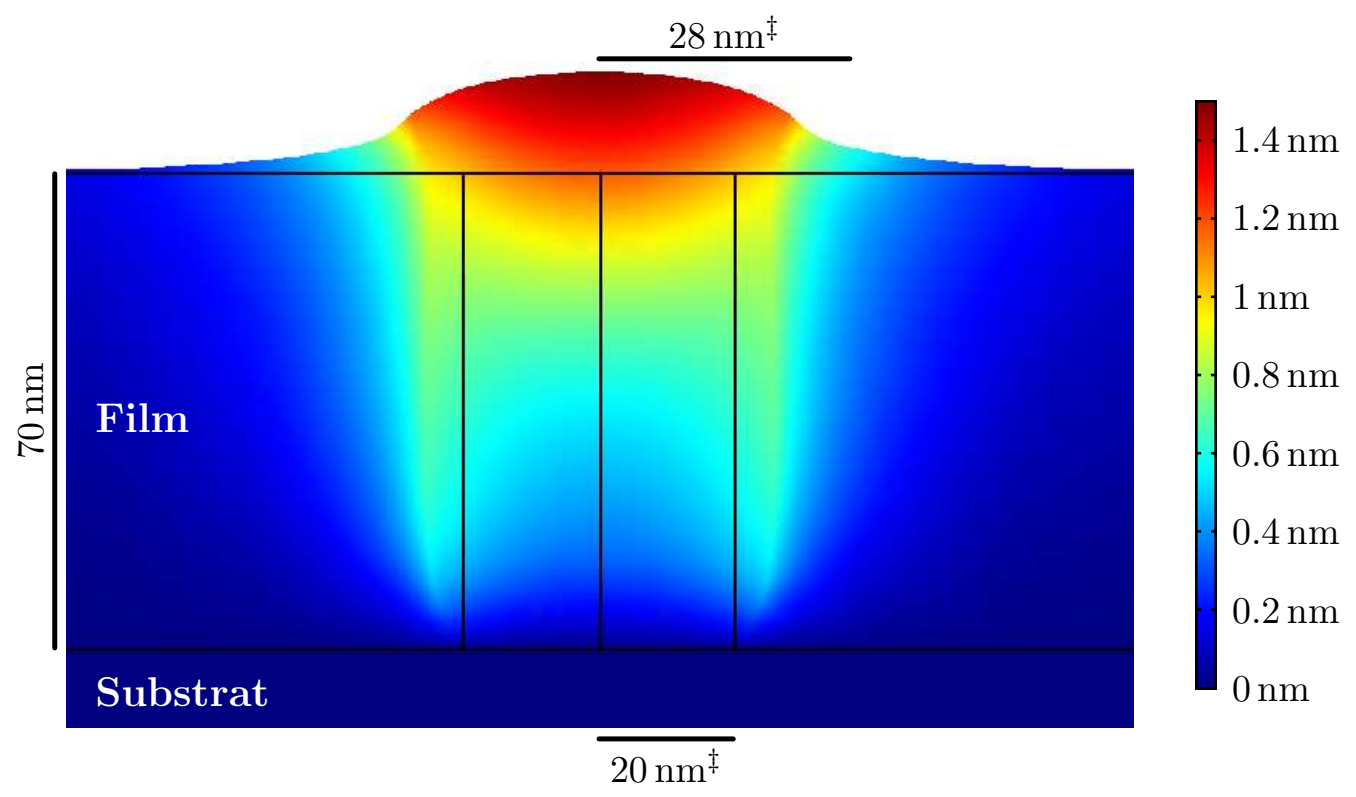

Abbildung 3.19: Totale Verschiebung einer zylinderförmigen Ausscheidung mit einem Radius von $20 \mathrm{~nm}$ in einem $70 \mathrm{~nm}$ Niobfilm, berechnet mit der Finite-Elemente-Methode. Die farbcodierte totale Verschiebung ist zehnfach überhöht dargestellt. Bei den blauen Bereichen tritt keine Verschiebung auf. In roter Farbe sind Verschiebungen von $1.5 \mathrm{~nm}$ gekennzeichnet.

geachtet, dass an den Probenrändern keine Ausdehnung in z-Richtung aufgetreten ist. Bei großen Ausscheidungen wurde das berechnete Gebiet entsprechend vergrößert.

Die Veränderung der Probe durch eine zylinderförmige Ausscheidung ist in Abb. 3.19 illustriert. Der Zylinder hat einen Radius von $20 \mathrm{~nm}$ und die Zylinderhöhe beträgt wie die Filmdicke $70 \mathrm{~nm}$. In der Abbildung ist die totale Verschiebung der Probe mit einem zweidimensionalen Schnitt farbcodiert dargestellt. Dabei entspricht die Farbe blau keiner Verschiebung und die maximale Verschiebung von $1.5 \mathrm{~nm}$ ist in rot abgebildet. Die Ausdehnung von Zylinder und Film wurde zehnfach überhöht dargestellt. Es ist zu erkennen, dass sich das Substrat, wie vorgegeben, nicht verändert. In einer Entfernung zur Mitte der Ausscheidung von ca. $100 \mathrm{~nm}$ ist im Film keine Verschiebung sichtbar. Die größte Dehnung von $1.5 \mathrm{~nm}$ in $z$-Richtung tritt in der Zylindermitte auf. Mit zunehmendem radialen Abstand von der Zylindermitte nimmt die Dehnung in $z$-Richtung ab. Der Zylinder dehnt sich radial aus und die maximale Dehnung in $x$ - und $y$-Richtung beträgt $0.8 \mathrm{~nm}$. Diese Dehnung verursacht die grünen zahnwurzelförmigen Veränderungen in der in Abb. 3.19 dargestellten totalen Verschiebung. Die langsam abnehmende Dehnung in z-Richtung bewirkt, dass die Ausscheidung an der Oberfläche größer erscheint als der Radius der Ausscheidung dies erwarten lässt.

\footnotetext{
¥Aufgrund der überhöhten Darstellung der Verschiebung sind die Scalen für $28 \mathrm{~nm}$ und $20 \mathrm{~nm}$ mit einer unterschiedlichen Vergrößerung dargestellt.
} 

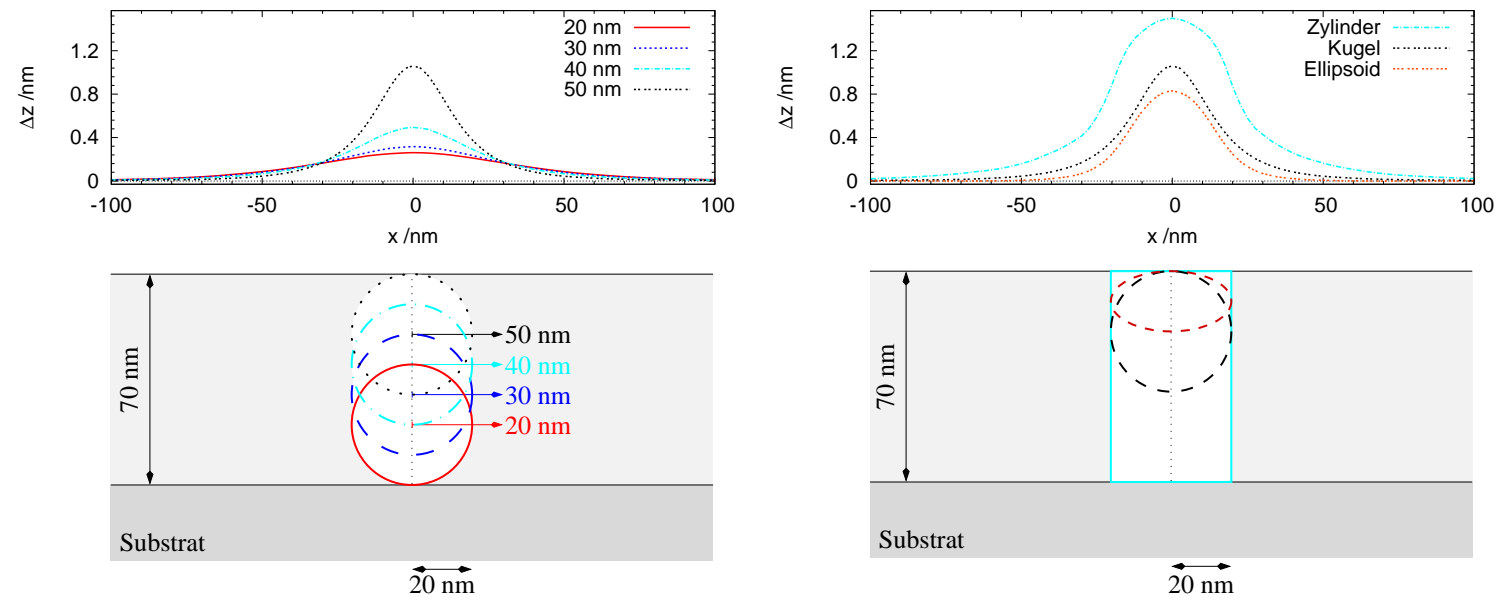

(a) Kugeln auf unterschiedlichen Höhen

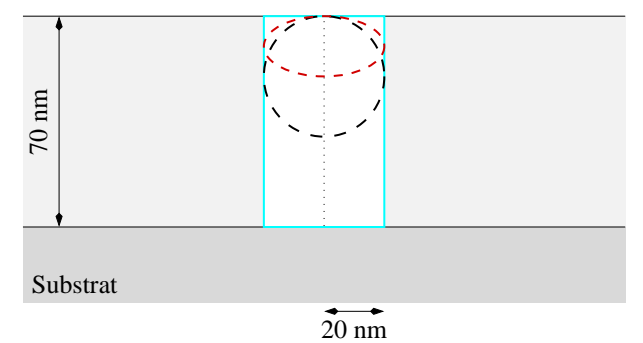

(b) Verschiedenen Formen an der Oberfläche

Abbildung 3.20: Höhenveränderungen an der Oberfläche hervorgerufen durch unterschiedliche Postionen und Formen der Ausscheidungen. In (a) sind die von unterschiedlich tief in der Schicht positionierten Kugeln (Radius $r=20 \mathrm{~nm}$ ) hervorgerufenen Ausdehnungen in $z$-Richtung an der Oberfläche dargestellt. Die größte Veränderung an der Oberfläche tritt auf, wenn die Ausscheidung direkt unter der Oberfläche sitzt. Bei den unterschiedlichen Formen in (b) wird deutlich, dass die größte Höhenzunahme bei zylinderförmigen Ausscheidungen zu erwarten ist.

Um die Berechnungen für unterschiedliche Ausscheidungsgrößen mit den bei STMMessungen erzielten Daten vergleichen zu können, ist eine Korrektur der Ausscheidungsgröße vorgenommen worden. Dabei ist der Radius bestimmt worden, bei dem die Ausdehnung in $z$-Richtung auf einen Wert $\Delta z_{\text {cut }}$ abgenommen hat. Ab einer bestimmten Höhe $\Delta z$ kann in den STM-Topographiebildern eine deutliche Unterscheidung zwischen Ausscheidung und der umgebenen $\alpha$-Phase vorgenommen werden. Diese Höhe ist als Grenzwert $\Delta z_{\text {cut }}$ festgelegt worden. Für große Ausscheidungen ist eine Höhe von $\Delta z_{\text {cut }}=0.5 \mathrm{~nm}$ gewählt worden. Ab einem Ausscheidungsradius von $r=15 \mathrm{~nm}$ ist der Wert langsam auf $\Delta z_{\text {cut }}=0.2 \mathrm{~nm}$ verringert worden. Wenn im Folgenden von einem Radius gesprochen wird, ist immer der Radius der an der Oberfläche sichtbaren Veränderung gemeint. Dieser ist für kleine Kugeln und Ellipsoide kleiner als der Ausscheidungsradius selbst. Ab einem Ausscheidungsradius von ca. $10 \mathrm{~nm}$ liegt der an der Oberfläche sichtbare Radius oberhalb des Ausscheidungsradius.

In Abb. 3.20 wird die Ausdehnung $\Delta z$ für verschiedene Formen und Positionen der Ausscheidungen verglichen. In (a) sind die Ausdehnungen von kugelförmigen Ausscheidungen mit einem Radius von $r=20 \mathrm{~nm}$ an unterschiedlichen Höhen in der Niobschicht zusammengestellt. Wenn sich die Ausscheidung direkt auf dem Substrat befindet $(z=20 \mathrm{~nm})$, gibt es an der Oberfläche die geringste Ausdehnung $\left(\Delta z_{\max }=0.26 \mathrm{~nm}\right)$. Beim Annähern der Ausscheidung an die Filmoberfläche wächst die Ausdehnung streng monoton an. Eine Ausscheidung an der Filmoberfläche 


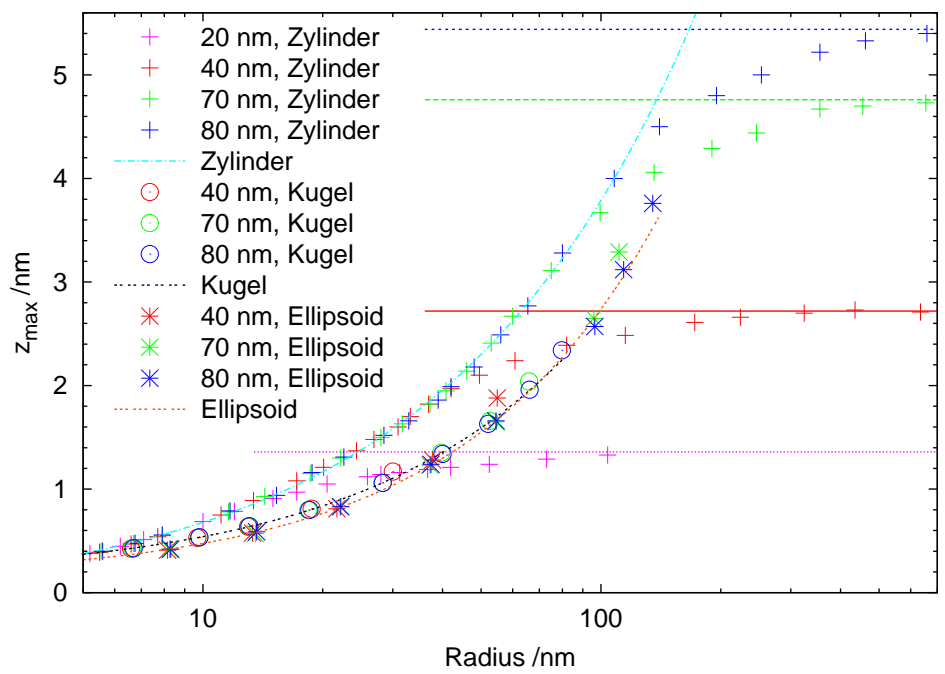

Abbildung 3.21: An der Oberfläche sichtbare Höhenveränderung unterschiedlicher Ausscheidungsformen. Dargestellt ist die Abhängigkeit der Maximalhöhe vom Radius der an der Oberfläche sichtbaren Ausscheidung. Dabei sind unterschiedliche Geometrien und Schichtdicken berücksichtigt worden. Bei Radien, die kleiner als die Filmdicke sind, zeigt sich keine Schichtdickenabhängigkeit der Höhenänderung $\Delta z$. Dieses wird durch die Anpassungen an die verschiedenen Geometrien deutlich. Bei zylinderförmigen Ausscheidungen nähert sich der Verlauf für große Radien dem theoretisch erwarteten Wert, der von der Schichtdicke abhängt, an. Für Kugeln und Ellipsoide werden diese Höhenänderungen nicht erreicht.

$(z=50 \mathrm{~nm})$ hat die größte Ausdehnung $\left(\Delta z_{\max }=1.06 \mathrm{~nm}\right)$. Dagegen wird gefunden, dass je tiefer die Ausscheidung in der Schicht liegt, um so größer ist das Gebiet an der Oberfläche, in dem noch Veränderungen zu sehen sind. Beim Vergleich von ellipsoiden, kugelförmigen und zylinderförmigen Ausscheidungen (der Radius bzw. die Halbachse in der $x$ - $y$-Ebene beträgt $20 \mathrm{~nm}$ ) in Abb. 3.20(b) ist zu sehen, dass mit zunehmender Ausdehnung der Ausscheidung in z-Richtung die maximale Veränderung an der Oberfläche steigt. Das gleiche Verhalten zeigt auch die laterale Größe der Veränderung. Die zylinderförmige Ausscheidung besitzt für alle $x$-Werte die größte Höhenzunahme. Bei den ellipsoiden Ausscheidungen zeigt sich stets die geringste Veränderung der drei Formen.

Die Rechnungen zu den unterschiedlichen Filmdicken und Ausscheidungsformen sind in Abb 3.21 zusammengetragen. Dabei sind für die einzelnen Schichtdicken unterschiedliche Farben gewählt worden und die drei verschiedenen Formen sind durch unterschiedliche Symbole gekennzeichnet. Aufgetragen ist die maximale Höhe der Ausscheidung in Abhängigkeit vom Radius der an der Oberfläche sichtbaren Veränderung. Mit vier waagerechten Linien sind die nach der linearen Elastizitätstheorie (vgl. Gl. (2.10), S 8) erwarteten Werte der Höhenänderung markiert, wenn in z-Richtung keine Spannungen vorhanden sind $\left(\sigma_{z}=0\right)$. Es ist zu erkennen, dass nur für zy- 
linderförmigen Ausscheidungen dieser Wert erreicht wird. Für die unterschiedlichen Geometrien lässt sich der Verlauf unabhängig von der Schichtdicke wie eingezeichnet anpassen. Diese Anpassung ist bei zylinderförmigen Ausscheidungen bis zu einem von der Schichtdicke abhängigen Radius möglich. Dieser Radius steigt mit zunehmender Schichtdicke. Wenn die schichtdickenunabhängige Anpassung bei zylinderförmigen Ausscheidungen nicht mehr möglich ist, nähert sich die maximale Höhe dem nach der linearen Elastizitätstheorie erwarteten Wert von $\Delta z_{\max }=0.068 \cdot h_{0}$ an.

Kugeln und Ellipsoide können bis zu dem Radius vergrößert werden, bei dem sie in $z$-Richtung durch die komplette Schicht reichen. Ab diesem Radius können dann keine Daten mehr bestimmt werden. Der größte dargestellte Radius entspricht somit genau der Ausscheidungsgröße, bei dem die Ausscheidung in $z$-Richtung durch die komplette Schicht reicht. Diese größtmögliche Ausscheidung hat eine etwas größere Höhe als dies der Trend durch die markierte Kurve erwarten lässt, was darauf zurückzuführen ist, dass kein Material zwischen Ausscheidung und Substrat ist, das verformt werden kann, wenn die Ausscheidung durch die komplette Schicht reicht.

Es ist zu erkennen, dass eine Schichtdickenabhängigkeit nur bei zylinderförmigen Ausscheidungen für große Radien vorhanden ist. Diese wird sichtbar, wenn sich die Ausscheidungshöhe dem Maximalwert nach der linearen Elastizitätstheorie für homogene Beladung (vgl. Abschnitt 2.2.2, S. 7) nähert. Die zylinderförmigen Ausscheidungen zeigen bei gleichem Radius die stärkste Veränderung in der Höhe. Die geringste Veränderung tritt bei Ellipsoiden auf. Der Unterschied zwischen Kugeln und Ellipsoiden ist gering $(<0.1 \mathrm{~nm})$.

Mit Hilfe der Finite-Elemete-Methode kann die gespeicherte elastische Energie pro Volumen berechnet werden. Nach Intergration über das Volumen einer Ausscheidung kann die gesamte gespeicherte elastische Energie dieser Ausscheidung ermittelt werden. In Abb. 3.22 ist diese Energie, normiert auf die Anzahl der Grenzflächenatome ${ }^{\S}$, für zylinderförmige Ausscheidung als Funktion des Radius für verschiedene Schichtdicken aufgetragen. Dabei ist der Abstand zweier Ebenen von $(a / \sqrt{2})$ gewählt worden. Die Atomdistanz ist in diesen Ebenen auf $a$, der Gitterkonstanten von Niob, festgelegt worden. Diese Abstände wurden aufgrund der (110)-Textur der Schicht gewählt. Durch diese Normierung wird der Unterschied zwischen den verschiedenen Schichtdicken besonders deutlich. In der Darstellung ist zu erkennen, dass für einen Radius von $r=5 \mathrm{~nm}$ die Energie pro Grenzflächenatom unabhängig von der Schichtdicke ist und bei ca. $0.1 \mathrm{eV} /$ Atom liegt. Die spezifische Energie steigt zu Beginn unabhängig von der Schichtdicke an. Bei größeren Radien ist eine Schichtdickenabhängigkeit zu erkennen: Je dicker der Film desto mehr spezifische Energie pro Oberflächenatom ist in der Ausscheidung gespeichert.

Da in diesen Berechnungen kein Spannungsabbau durch Gleiten von Ausscheidung und Film gegeneinander zugelassen ist, sind diese beschriebenen Ergebnisse für kohärente Ausscheidungen bestimmt worden. Wenn Versetzungen gebildet wer-

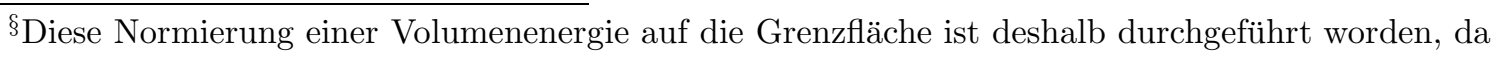
die berechnete Energie später mit der Linienenergie von Versetzungen verglichen wird. 


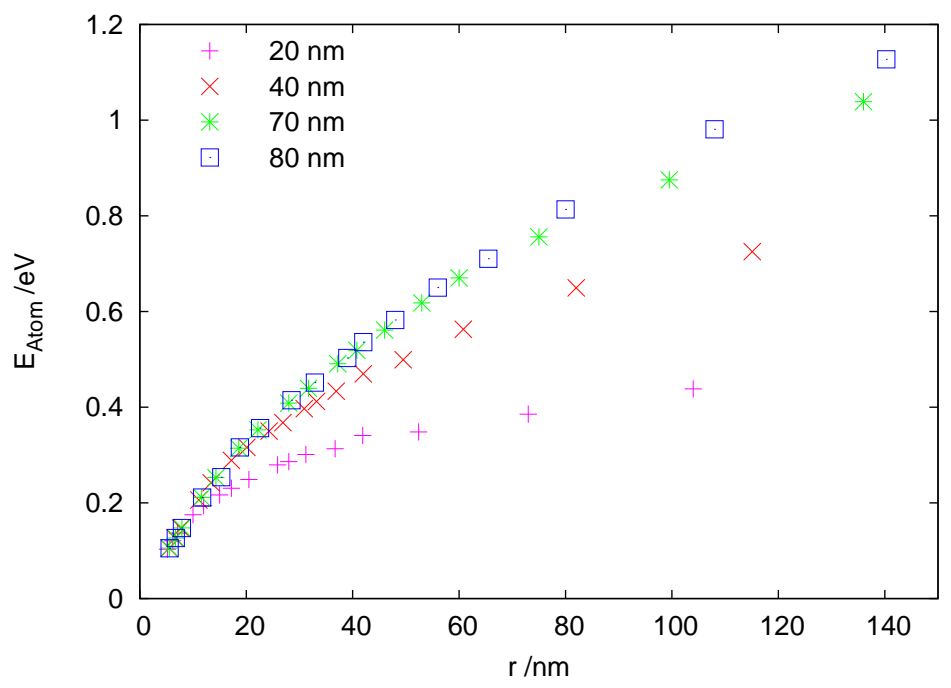

Abbildung 3.22: Elastische Energie, die in zylinderförmigen Ausscheidungen gespeichert ist. Aufgetragen wurde die elastische Energie in Abhängigkeit vom Radius. Die elastische Energie wurde mit Hilfe der Finite-Elemente-Methode bestimmt. Dabei wurde über die Ausscheidung integriert und dieses Integral auf die Atomzahl der Grenzfläche von Film und Ausscheidung normiert.

den, kommt es zu einer Verschiebung zwischen Film und Ausscheidung. Ist keine Kopplung in z-Richtung zwischen Ausscheidung und Film vorhanden, beträgt die Höhenänderung für zylinderförmige Ausscheidungen $\Delta z=0.068 \cdot h_{0}$ (vgl. lineare Elastizitätstheorie Abschnitt 2.2.2, S. 7) und ist unabhängig von der Ausscheidungsgröße.

\subsubsection{Ausscheidung in einer Drahtgeometrie}

Die Charakterisierung der hergestellten Schichten (s. Abschnitt 3.2.2, S. 25) hat gezeigt, dass nicht alle untersuchten Proben eine geschlossene Schicht aufweisen. Bei einer Schichtdicke von $20 \mathrm{~nm}$ treten mäanderförmige Oberflächenstrukturen auf (vgl. Abb. 3.10, S. 28). Um diese Struktur mit der Finite-Elemente-Methode zu beschreiben, wurde diese komplizierte Form durch eine einfache Drahtgeometrie angenähert. Als Geometrie zur Berechnung ist ein $300 \mathrm{~nm} \times 70 \mathrm{~nm} \times 20 \mathrm{~nm}$ großer Niobdraht auf einem starren Saphirsubstrat ausgewählt worden, in dessen Mitte sich eine $70 \mathrm{~nm} \times 70 \mathrm{~nm} \times 20 \mathrm{~nm}$ große Hydridausscheidung befand, die sich um $2.9 \%$ in alle drei Richtungen ausdehnt. Die Randbedingungen sahen dabei wie folgt aus:

- Im Substrat sind keine Dehnungen zugelassen.

- Zwischen Substrat, Draht und Ausscheidung ist jeweils kein Abgleiten erlaubt.

- Der Draht darf sich in $x$-Richtung (lange Achse) nicht über das Substrat ausdehnen. 
- In $y$ - und $z$-Richtung sind für den Draht keine Einschränkungen vorgegeben.

- Die Ausscheidung hat keine Einschränkungen in alle drei Raumrichtungen.

Abb. 3.23 zeigt das mit der Finite-Elemente-Methode berechnete Gebiet für die Drahtgeometrie. Der Draht erstreckt sich in $x$-Richtung über das gesamte Substrat, in $y$-Richtung ist der Draht auf $70 \mathrm{~nm}$ beschränkt. In der Drahtmitte befindet sich die quaderförmige Ausscheidung die sich in $y$ - und $z$-Richtung über den kompletten Draht erstreckt.

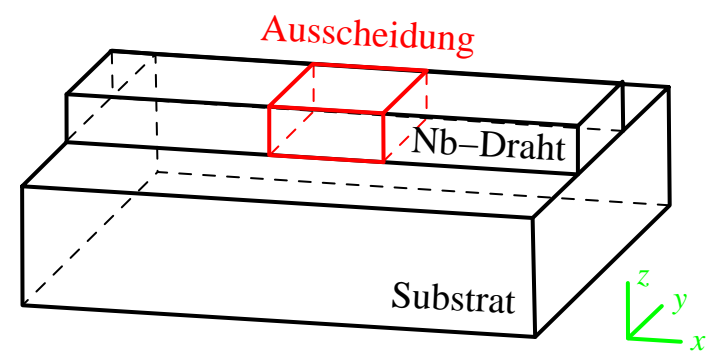

Abbildung 3.23: Berechnetes Gebiet der FiniteElemente-Methode für die Drahtgeometrie. Diese in rot eingezeichnete Struktur dehnt sich um $2.9 \%$ in alle drei Raumrichtungen aus.

In Abb. 3.24 ist das Ergebnis dieser Berechnung dargestellt. Abgebildet ist die Verschiebung in $z$-Richtung in einer Richtung senkrecht und parallel zur Drahtrichtung. Die maximale Erhöhung der Probe beträgt $1.1 \mathrm{~nm}$, was in dem Höhenprofil in

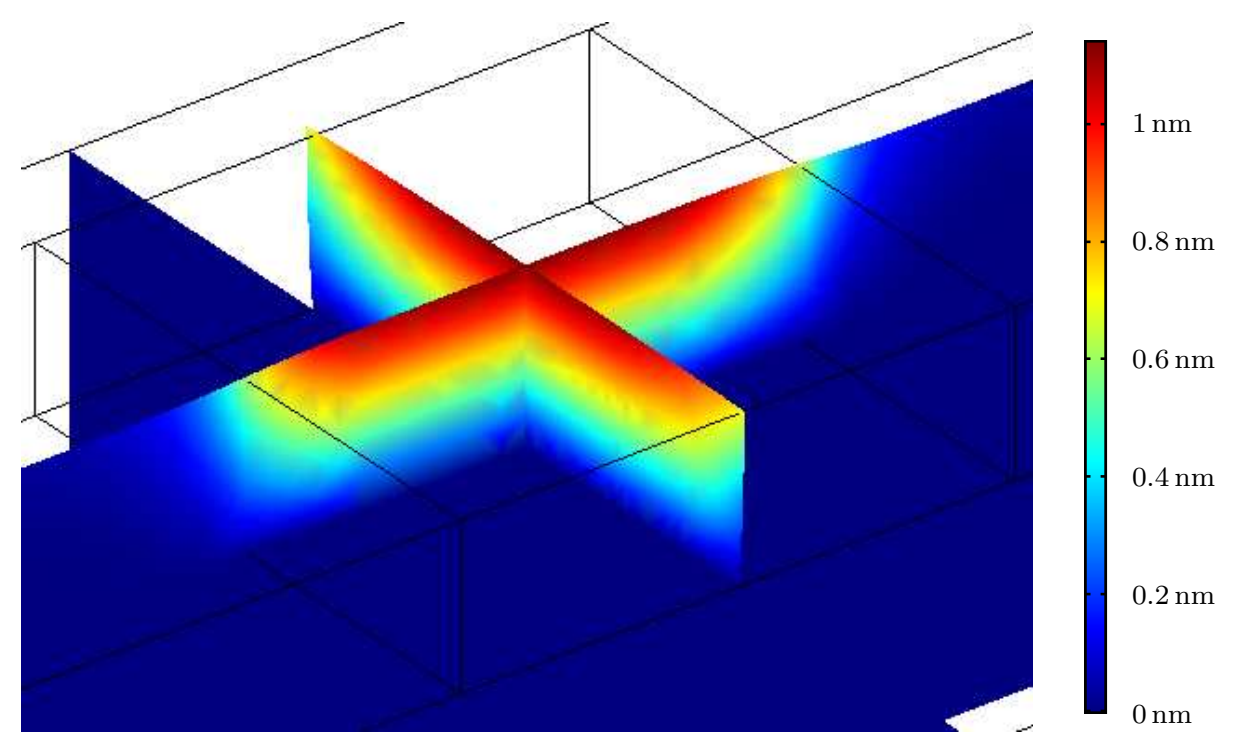

Abbildung 3.24: Dehnung eines Niobdrahts mit einer quaderförmigen Ausscheidung. Berechnet wird eine würfelförmige Ausscheidung mit den Ausmaßen von $70 \mathrm{~nm} \times 70 \mathrm{~nm} \times$ $20 \mathrm{~nm}$ in einem $300 \mathrm{~nm} \times 70 \mathrm{~nm} \times 20 \mathrm{~nm}$ kleinen Draht. Es wird eine Vergrößerung der Ausscheidung von $2.9 \%$ in alle Raumrichtungen vorgegeben und die resultierende Dehnung in $z$-Richtung in zwei senkrecht aufeinander stehenden Ebenen dargestellt. Die maximale Verschiebung in $z$-Richtung beträgt $1.1 \mathrm{~nm}$ (vgl. Abb. 3.25(a)). Zusätzlich ist zu erkennen, dass sich die Ausscheidung in $y$-Richtung über die ursprüngliche Größe ausgedehnt hat. Diese Verschiebung beträgt $1.1 \mathrm{~nm}$ in beide Richtungen (vgl. Abb. 3.25(b)). 


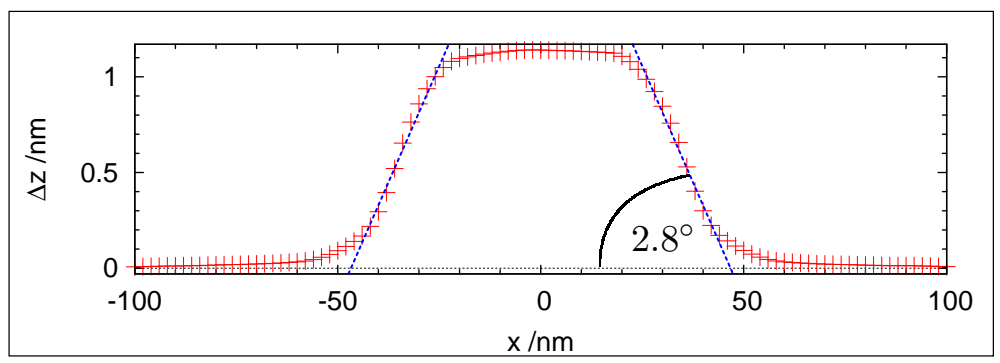

(a) parallel zur Drahtachse

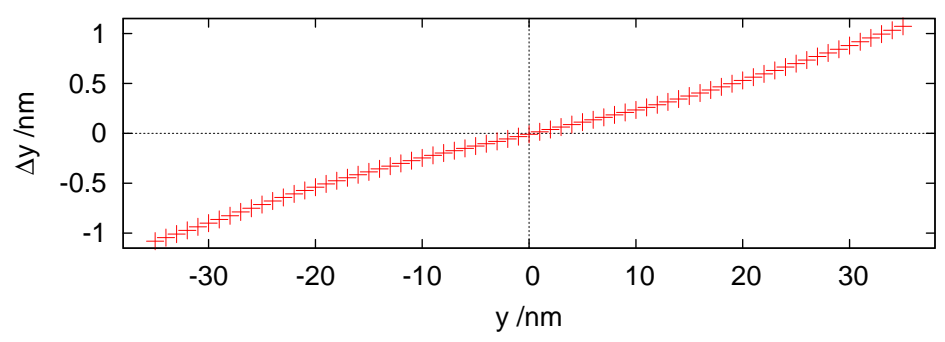

(b) senkrecht zur Drahtachse

Abbildung 3.25: Höhenprofile der Ausdehnung bei einer Drahtgeometrie. In (a) ist die Ausdehnung in $z$-Richtung parallel zur Drahtachse dargestellt. Die maximale Erhöhung beträgt $1.1 \mathrm{~nm}$ und der Anstiegswinkel von der $\alpha$-Phase zum Hydrid ist 2.8(1) . Die Vergrößerung senkrecht zur Drahtachse ist in (b) zu sehen. Die Veränderung an den Drahträndern $(y= \pm 35 \mathrm{~nm})$ ist $\Delta y= \pm 1.1 \mathrm{~nm}$. Der Draht dehnt sich somit über die beiden ursprünglichen Ränder aus.

Abb. 3.25(a) deutlich wird. Der Anstiegswinkel von der $\alpha$-Phase zur Hydridausscheidung beträgt $2.8(1)^{\circ}$. Dies ist im Höhenprofil mit blauen Linien markiert. Außerdem ist aus dem Höhenprofil in Abb. 3.25(b) abzulesen, dass sich der Draht in $y$-Richtung über die ursprüngliche Drahtgeometrie ausgedehnt hat. Diese Dehnung beträgt an den Drahträndern $(|y|=35 \mathrm{~nm})$ in beide Richtungen $|\Delta y|=1.1 \mathrm{~nm}$.

In diesem Kapitel ist gezeigt worden, unter welchen Bedingungen die epitaktischen Niobschichten hergestellt und untersucht wurden. Die Schichteigenschaften sind dargestellt worden. Außerdem sind mit der Finite-Elemente-Methode die Veränderungen, die Ausscheidungen im Volumen an der Oberfläche erzeugen, berechnet worden. Im folgenden Kapitel werden die im Experiment beobachteten Veränderungen während der Wasserstoffbeladung beschrieben. 


\section{Experimentelle Resultate}

In diesem Kapitel werden die Messungen dieser Arbeit vorgestellt. Die einzelnen Ergebnisse werden dabei unabhängig voneinander interpretiert. Die Diskussion und Interpretation verschiedener Teilaspekte, die in diesem Kapitel nacheinander behandelt werden, werden in Kap. 5 (s. S. 83) im Zusammenhang geführt.

Im Abschnitt 4.1 werden die Veränderungen in der $\alpha$-Phase während der Wasserstoffbeladung erläutert. Dort wird in Abschnitt 4.1.1 bzw. 4.1.2 die Geschwindigkeit der Wasserstoffaufnahme und -abgabe untersucht. Im Weiteren wird auf die Randlöslichkeit der $\alpha$-Phase sowie auf Veränderungen der Oberflächenmorphologie eingegangen. Im Abschnitt 4.2 wird dann die Hydridphase beschrieben. Es wird der Randbereich zwischen $\alpha$-Phase und Hydrid behandelt. Außerdem wird die Schichtdickenabhängigkeit der vertikalen lokalen Ausdehnung des Hydrids dargestellt. Des Weiteren wird gezeigt, dass bei der Hydridbildung zwei unterschiedliche Ausscheidungstypen entstehen. Diese beiden Typen werden untersucht und ihre Eigenschaften charakterisiert. In Abschnitt 4.3 werden Keimbildung und Wachstum untersucht. Dabei werden die Anfangsstadien und die Sättigung behandelt. In diesem Teil wird auch auf die Umwandlung der beiden Ausscheidungstypen eingegangen. In Abschnitt 4.4 wird eine Schicht mit einer offenen Oberflächenstruktur beschrieben. Es wird sich zeigen, dass bei dieser Oberflächenmorphologie auch eine laterale Schichtausdehnung möglich ist.

\subsection{Wasserstoffaufnahme in der $\alpha$-Phase}

\subsubsection{Kinetik bei der Beladung}

Um die Kinetik der Wasserstoffaufnahme der Niobschicht zu bestimmen, wird die in Abschnitt 3.2.4 (s. S. 30) beschriebene Messung ausgewertet. Dort ist bei geringen Substratdicken eine große Höhenänderung bei der Wasserstoffzugabe gemessen worden. Diese ist hauptsächlich auf die Verbiegung des Substrates zurückzuführen (siehe Tab. 3.1, S. 35). Die zeitliche Entwicklung der Wasserstoffbeladung ist mit dieser Höhenänderung korreliert, unabhängig davon, ob die Änderung durch Filmexpansion oder Substratkrümmung hervorgerufen wird. In Abbildung 3.13 (s. S. 30) ist die zeitliche Entwicklung der Höhe eines $100 \mathrm{~nm}$ Nb-Films als topographisches STMBild, sowie fünf Höhenprofile dargestellt. Wie schon in Gl. (3.4) (s. S. 31) angegeben, nimmt die Höhe mit dem exponentiellen Faktor $\left(1-e^{-m t}\right)$ zu. Die Zeitabhängigkeit wird durch einen Wert von $m=6.3(1) \cdot 10^{-3} \mathrm{~s}^{-1}$ beschrieben. Eine solche exponenti- 
Tabelle 4.1: Zeitabhängigkeit der Wasserstoffaufnahme bei verschiedenen Drücken und Schichtdicken

\begin{tabular}{|c|c|c|c|c|}
\hline$h_{0}[\mathrm{~nm}]$ & Startdruck $[\mathrm{mbar}]$ & Enddruck $[\mathrm{mbar}]$ & $m\left[10^{-3} / \mathrm{s}\right]$ & $D_{\text {eff }} \mathrm{cm}^{2} / \mathrm{s}$ \\
\hline 100 & $3 \cdot 10^{-10}$ & $2 \cdot 10^{-8}$ & $6.3(1)$ & $3.99(8) \cdot 10^{-13}$ \\
\hline 50 & $4.2 \cdot 10^{-10}$ & $1.6 \cdot 10^{-7}$ & $11.1(1)$ & $1.77(2) \cdot 10^{-13}$ \\
\hline 50 & $1.6 \cdot 10^{-7}$ & $1.4 \cdot 10^{-6}$ & $5.5(3)$ & $8.8(4) \cdot 10^{-14}$ \\
\hline
\end{tabular}

elle Zeitabhängigkeit wurde in den Abbildungen 3.14, 3.15(b) und 3.16(b) (s. S. 31 - 33) eingezeichnet. Die Werte von $m$ sind in Tabelle 4.1 zusammengestellt. Dort ist auch die effektive Diffusionskonstante $D_{\text {eff }}$ aufgeführt, die über die Gl. (2.29) (s. S. 15) berechnet worden ist. Die Werte für die berechnete Diffusionskonstante sind um mehrere Größenordnungen kleiner als der von ZH. QI et.al. angegebene Wert für massives Niob von $D_{\mathrm{Nb}}=8.1 \cdot 10^{-6} \mathrm{~cm}^{2} / \mathrm{s}$ [QVLW83]. Die Diffusion von Wasserstoff in der Niobschicht ist somit nicht der zeitlimitierende Faktor.

Weiterere, die Rate bestimmende, Faktoren sind die Menge des angebotenen Wasserstoffs, der die Oberfläche erreicht, der Bruchteil der Wasserstoffmoleküle, die an der Oberfläche dissoziieren und weitere Oberflächenbarrieren (z. B. Verunreinigungen, die die Wasserstoffaufnahme hemmen). Bei einem Druck von $1 \cdot 10^{-6}$ mbar trifft durch die Atombewegung eine Monolage Wasserstoff $\left(\mathrm{ML}_{\mathrm{H}}\right)$ pro Sekunde auf eine Oberfläche (vgl. Theorie der Gaskinetik z. B. in [Ohr02, Kapitel 2.2]). Der Druck und die Zeit gehen linear in die auf die Oberfläche zugeführte Gasmenge ein. Aber nicht alle Moleküle bzw. Atome, die auf die Oberfläche treffen, werden auch auf der Oberfläche adsorbiert. Diese Effekte an der Probenoberfläche werden zum Stickingfaktor $s$ zusammengefasst.

Als Stickingfaktor $s$ wird der Anteil bezeichnet, der von den auf die Oberfläche treffenden Molekülen auch auf der Oberfläche verbleibt. Um den Stickingfaktor des Wasserstoffs zu berechnen, wird die Zeit benötigt, bei der eine bekannte Menge in die Schicht gelangt ist. Mit dem STM ist es möglich, die Phasenumwandlung von der $\alpha$-Phase zum Hydrid zu beobachten. Die Konzentration, bei der diese Umwandlung stattfindet, ist von M. Dornheim gemessen worden und liegt bei circa $c_{\mathrm{H}}=0.1$ [Dor02]. In Abb. 3.16(b) (s. S. 33) sind fünf Höhenprofile eines $50 \mathrm{~nm}$ dünnen Niobfilms bei der Wasserstoffbeladung mit einem Druck von $1.4 \cdot 10^{-6}$ mbar dargestellt. Das in blau gezeichnete Profil weist eine große Stufe 340 s nach der Wasserstoffzugabe auf, sowie eine kleinere Erhöhung nach 210 s. Diese Erhöhungen entstehen durch die Bildung des Hydrids. Der erste Höhenanstieg markiert damit den Zeitpunkt, bei dem die Konzentration $c_{\mathrm{H}}=0.1$ erreicht wird. So kann mit der Zeit, dem Druck, der Schichtdicke (Anzahl der Niobebenen $\mathrm{ML}_{\mathrm{Nb}}$ ) und der Konzentration $c_{\mathrm{H}}=0.1 \mathrm{H} / \mathrm{Nb}$ der mittlere Stickingfaktor $s$ berechnet werden:

$$
\begin{aligned}
& s=\frac{\text { sorbierter Wasserstoff }}{\text { angebotener Wasserstoff }}=\frac{\mathrm{ML}_{\mathrm{Nb}} \cdot c_{\mathrm{H}}}{\mathrm{ML}_{\mathrm{H}}} \approx \frac{16.5}{588} \\
& s \approx 3 \% .
\end{aligned}
$$


Von L. Johnson et.al. wurde der Stickingfaktor für Wasserstoff auf einer Nioboberfläche je nach Bedeckungsgrad von $1 \%$ bis $20 \%$ bestimmt [JDD72]. Der hier gemessene Wert mit unbekanntem Bedeckungsgrad liegt somit im Bereich der von L. Johnson et.al. veröffentlichten Werte. Das bedeutet, dass die angebotene Wasserstoffmenge bei geringen Gasdruck der Faktor ist, der die Kinetik der Wasserstoffaufnahme bestimmt.

\subsubsection{Kinetik bei der Entladung}

Wenn der Wasserstoffdruck in der Kammer wieder sinkt, entweicht der Wasserstoff aus der Probe, solange sich die Probe innerhalb der $\alpha$-Phase befindet. Dies ist daran zu erkennen, dass bei Absenkung des äußeren Wasserstoffdrucks die Krümmung des Substrates zurückgeht. Dies ist bei einer Messung an einem $50 \mathrm{~nm}$ dünnen Niobfilm beobachtet worden. Bei der Beladung bis zu einem Druck von $2.6 \cdot 10^{-8}$ mbar tritt eine Krümmung des Substrates auf. Wenn die Wasserstoffzufuhr gestoppt wird und so der Wasserstoffdruck in der Kammer abnimmt, nimmt die Schichthöhe mit dem Faktor $\left(1-\exp \left\{-2.5(1) \cdot 10^{-3} \cdot t / \mathrm{s}\right\}\right)$ ab. Diese langsame Abnahme der Probenhöhe ist in Abb. $4.1 \mathrm{zu}$ sehen. Die Abnahme der Höhe ist dadurch zu erklären, dass der Wasserstoff aus der Probe entweicht. Dabei verringert sich gleichzeitig die Spannung im Film, und die Krümmung nimmt ab. Die Zeitabhängigkeit der Entladung liegt in derselben Größenordnung wie bei der Beladung (vgl. Tab. 4.1). Nach G. Hörz und E. Fromm ist die Geschwindigkeit der Entladung durch die Rekombination der Wasserstoffmoleküle an der Oberfläche und anschließender Desorbtion bestimmt [HF76].

\subsubsection{Maximaldruck der $\alpha$-Phase bei Raumtemperatur}

Im Folgenden wird auf die Thermodynamik, speziell die Schichtdickenabhängigkeit des Maximaldrucks der Existenz der $\alpha$-Phase* eingegangen. Dazu ist in Abb. 4.2 (s. S. 49) für verschiedene Schichtdicken der Druck aufgetragen, bei dem sich das Hydrid gebildet hat. Da der Wasserstoffdruck während der Messung nicht kontinuierlich, sondern stufenweise erhöht wurde, kann für den Druck, bei dem das Zweiphasengebiet beginnt, für jede Schichtdicke nur ein Bereich festgelegt werden. Die Drücke, bei dem Hydridausscheidungen beobachtet wurden, sind durch Kreuze markiert. Des Weiteren sind für die unterschiedlichen Schichtdicken die höchsten Drücke, bei dem noch kein Hydrid zu sehen war, als Kreise in den Graphen eingetragen. Der Druck, bei dem die Phasenumwandlung startet, liegt also für die unterschiedlichen Schichtdicken zwischen diesen beiden Drücken. Eine Schichtdickenabhängigkeit ist für Schichtdicken $d>35 \mathrm{~nm}$ nicht zu beobachten. Hier beschreibt ein konstanter Druck von $6(1) \cdot 10^{-7}$ mbar alle gemessenen Punkte. Dieser konstante Wert ist als Gerade mit in den Graphen eingezeichnet. Dieser Wert liegt etwas unterhalb von $1.3 \cdot 10^{-6} \mathrm{mbar}$, den von E. Hörz und E. Fromm für massives Niob-Wasserstoff bei $25^{\circ} \mathrm{C}$ angegebe-

\footnotetext{
${ }^{*}$ Hier ist der Maximaldruck gemeint, bei dem sich noch keine Hydridphase bildet.
} 


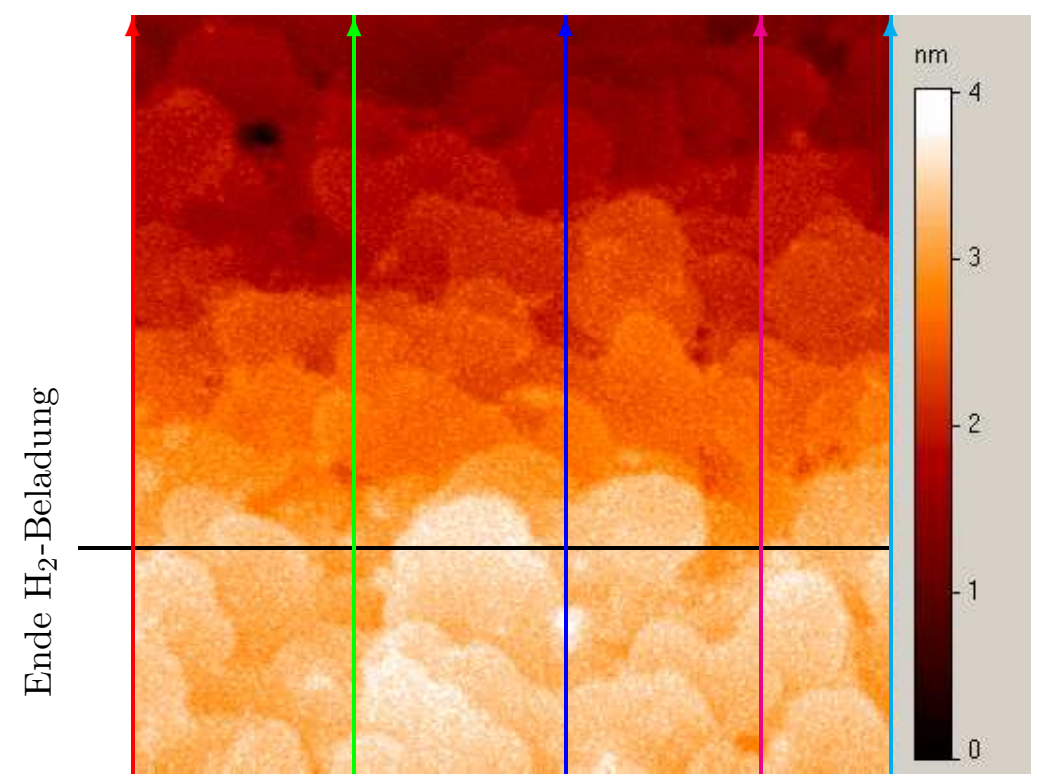

(a) STM-Bild, Größe: $500 \times 500 \mathrm{~nm}^{2}$

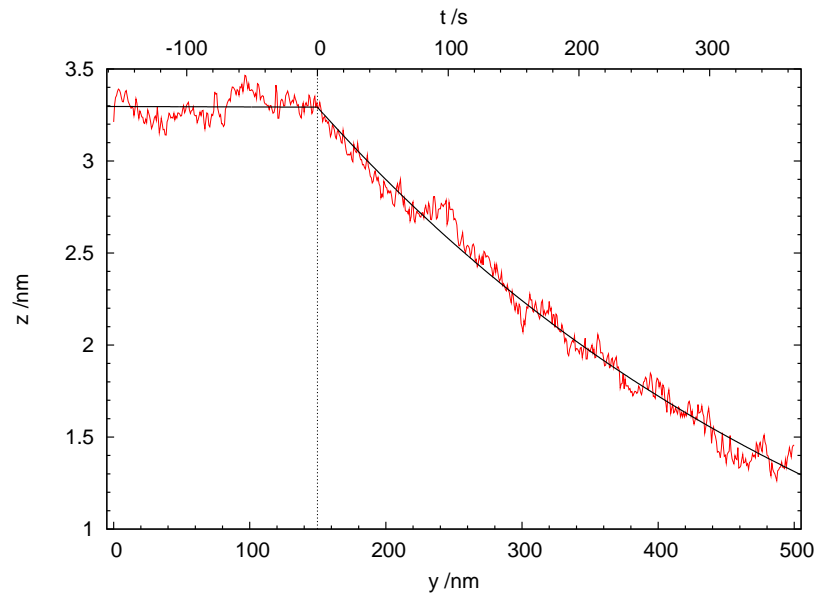

(b) Höhenprofil (Mittelwert der in (a) eingezeichneten Pfeile)

Abbildung 4.1: Desorbtion von Wasserstoff bei Druckerniedrigung. Zu Beginn der STMMessung des $50 \mathrm{~nm}$ dünnen Niobfilm (Substratmiscut $\vartheta<0.1^{\circ}$ ) lag der Wasserstoffdruck bei $2.6 \cdot 10^{-8}$ mbar. Die Wasserstoffbeladung ist beim Scannen der markierten Bildzeile gestoppt worden. Mit der daraus folgenden Druckabnahme nimmt die Schichthöhe mit dem zeitabhängigen Faktor $\left(1-\exp \left\{-2.5(1) \cdot 10^{-3} \cdot t / \mathrm{s}\right\}\right)$ ab. Dieser Verlauf ist in das Höhenprofil (b) eingezeichnet. Das Höhenprofil ist die Mittelung über die fünf in (a) eingezeichneten Pfeile.

nen Wert [HF76G Ger von J. A. PRYde und C. G. Titcomb bei einer Temperatur von $22{ }^{\circ} \mathrm{C}$ gemessene Wert für massive Proben liegt mit $2.3 \cdot 10^{-7}$ mbar unterhalb des hier bestimmten Wertes [PT69]. Es hat sich somit gezeigt, dass für Schichtdicken 


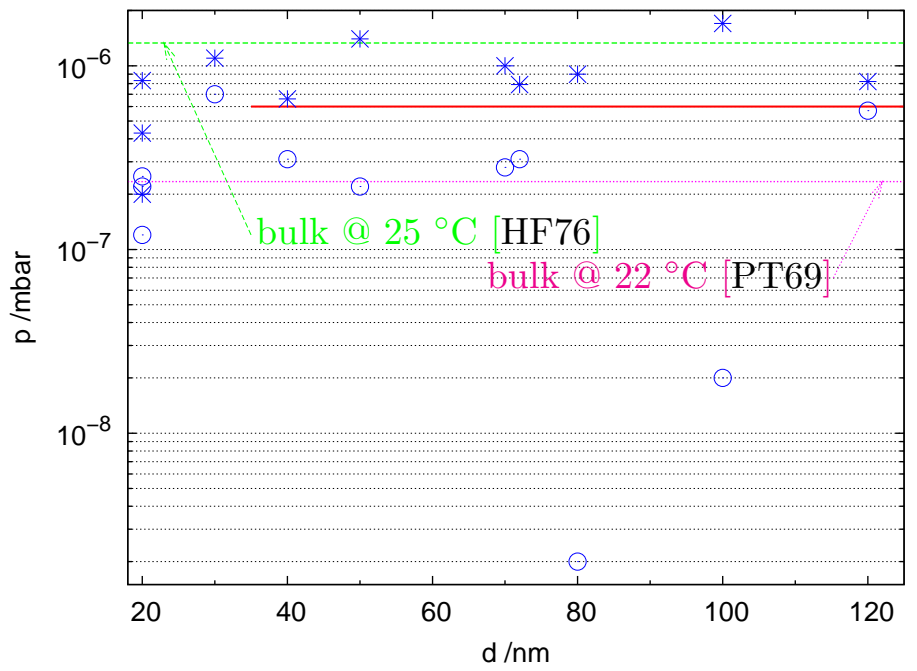

Abbildung 4.2: Wasserstoffdruck, bei dem sich für unterschiedliche Schichtdicken das Hydrid bildet. In Abhängigkeit von der Schichtdicke ist der Druck aufgetragen, bei dem nach stufenweiser Druckerhöhung, die Hydridausscheidungen sichtbar wurden. Mit Kreuzen sind die Drücke eingezeichnet, bei dem das Hydrid entstanden ist. Die Kreise zeigen den Druck, bei dem nur die $\alpha$-Phase zu sehen ist. Mit einer grünen Linie ist der von E. HöRZ angegebene Wert für bulk von $1.3 \cdot 10^{-6}$ mbar bei $25{ }^{\circ} \mathrm{C}$ markiert [HF76]. In magenta ist der von J. A. PRYDE bei einer Temperatur von $22^{\circ} \mathrm{C}$ gemessene Wert von $2.3 \cdot 10^{-7}$ mbar eingezeichnet [PT69].

zwischen $35 \mathrm{~nm}$ und $120 \mathrm{~nm}$ keine signifikante Schichdickenabhängigkeit der Wasserstoffrandlöslichkeit vorhanden ist.

\subsubsection{Oberflächenmorphologie innerhalb der $\alpha$-Phase}

Bei der Wasserstoffbeladung einer Schicht entstehen große mechanische Spannungen (vgl. Abschnitt 2.2.4, s. S. 9). Sie können durch plastische Verformung abgebaut werden. Über diese durch Wasserstoff induzierte Bildung von Versetzungen wird in der Literatur mehrfach berichtet [SW78, LPB $\left.{ }^{+} 99\right]$. U. LAUDAHN et.al. haben in ihren Spannungsmessungen plastische Verformung innerhalb der $\alpha$-Phase angenommen $\left[\mathrm{LPB}^{+} 99\right]$. J. Č́̌́̌EK et.al. hingegen finden keinen Hinweis auf Versetzungen in der $\alpha$-Phase $\left[\mathrm{CMP}^{+} 06, \mathrm{CPB}^{+} 06 \mathrm{a}, \mathrm{CPB}^{+} 06 \mathrm{~b}\right]$. Diese beiden unterschiedlichen Ergebnisse sollen hier geklärt werden. Es wird untersucht, ob in epitaktischen Schichten plastische Verformungen und Versetzungen in der $\alpha$-Phase auftreten.

In den hergestellten Filmen sind intrinsische Schraubenversetzungen zu sehen, die als Enden von Terrassenstufen erkennbar sind (vgl. Abb. 3.9, S. 27). Bei der Beladung mit Wasserstoff (vgl. Abb. 3.13, 3.15 und 3.16, S. 30 - 33) entstehen keine weiteren Versetzungen in der $\alpha$-Phase. Es sind weder zusätzliche Schraubenversetzungen noch Stufenversetzungen sichtbar. Es entstehen keine weiteren Versetzungen, da die Bela- 


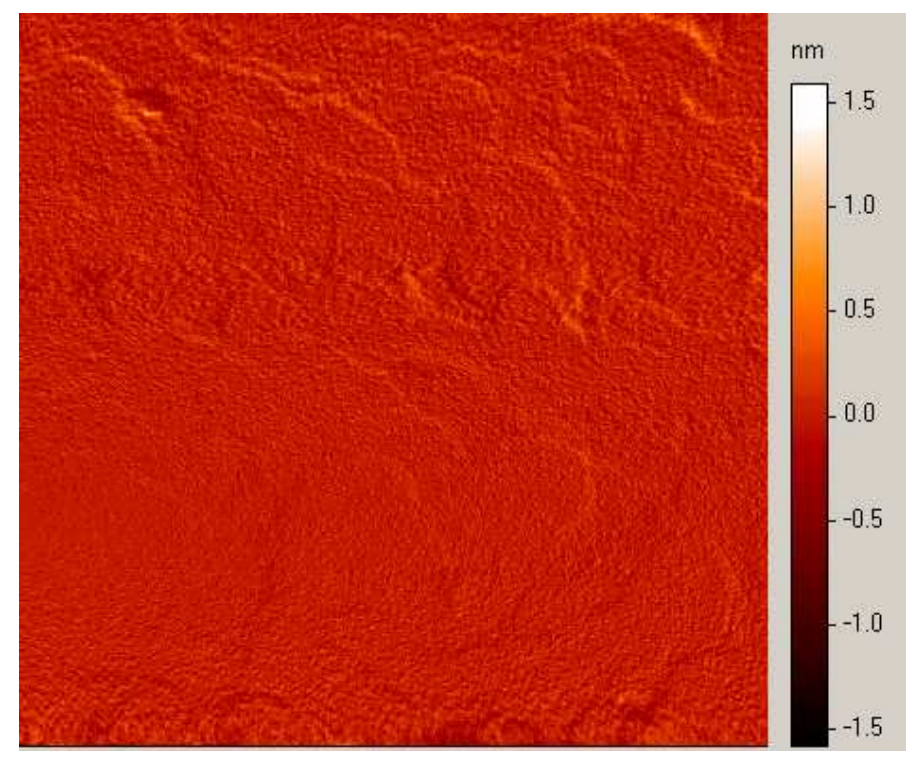

Abbildung 4.3: Differenzbild eines $50 \mathrm{~nm}$ dünnen Niobfilms auf einem Substrat mit einem Miscut kleiner als $0.1^{\circ}$. Dargestellt ist der Unterschied zwischen einem Bild während der Wasserstoffbeladung und einem nach der Beladung. Wie die gleichmäßige Farbkodierung zeigt ist eine Änderung der Oberflächenmorphologie ist nicht festzustellen. (Größe: $495 \times$ $\left.481 \mathrm{~nm}^{2}\right)$.

dung innerhalb der $\alpha$-Phase reversibel ist. Die Reversibilität zeigt sich in Abb. 4.3. Dort ist ein Differenzbild von zwei STM-Bildern dargestellt. Das erste Bild wurde vor der Wasserstoffbeladung aufgenommen, das zweite nach der Beladung. Mit einer Kreuzkorrelation ist die Drift während der Beladung bestimmt worden, um dann die Differenz der leicht gegeneinander verschobenen Bilder auszurechnen. Das Differenzbild in Abb. 4.3 zeigt keine gravierenden Unterschiede, nur kleinere Differenzen sind zu sehen. Diese rühren zum einen daher, dass die Aufnahme nicht kontinuierlich ist, sondern aus Pixeln aufgebaut ist, zum anderen daher, dass die Aufnahmen in einem längerem Zeitraum (hier 8 min) aufgenommen wurden. Da die Probe kontinuierlich driftet, ist die Drift somit für jeden Messpunkt unterschiedlich. Beim Vergleich von zwei diskreten Messreihen ist es aber nur möglich, alle Punkte eines Datensatzes gleich zu behandeln, da sonst einige Punkte keinen Vergleichswert hätten. Bei der in der Abbildung gewählten Driftkorrektur ist im unteren Bildteil ein Gebiet mit geringem Unterschied zwischen den STM-Bildern zu erkennen. Mit einer anderen Driftkorrektur kann dieser Bereich über das gesamte Bild verschoben werden. Eine Veränderung der Oberflächenmorphologie zwischen der unbeladenen, der beladenen und der entladenen Schicht ist daher nicht festzustellen. Die Oberflächenunebenheit verändert sich bei der Wasserstoffbeladung nicht. Der RMS-Wert beträgt für einen $100 \mathrm{~nm}$ dünnen Niobfilm in einem $500 \times 500 \mathrm{~nm}^{2}$ großen Bereich vor und nach der Wasserstoffbeladung $0.42 \mathrm{~nm}$. Damit ist die Beladung innerhalb der $\alpha$-Phase reversibel, was die Bildung von weiteren Versetzungen während der Beladung in der $\alpha$-Phase ausschließt. 


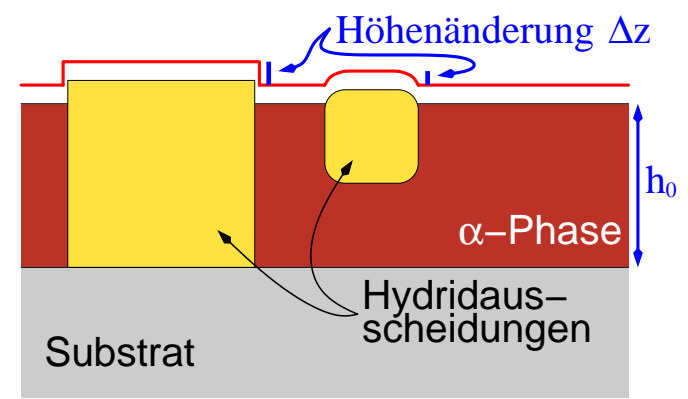

Abbildung 4.4: Hydridbildung in dünnen Schichten. Wenn sich lokal ein Hydrid bildet, ist dies an der Oberfläche sichtbar. Durch die unterschiedliche Gitterdehnung in der Schicht ( $\alpha$-Phase) und der Ausscheidung (Hydrid) bilden sich an der Oberfläche Stufen $\Delta z$, die in der STM-Messung (rote Linie) sichtbar sind.

\subsection{Hydridbildung in epitaktischen Schichten}

Aufgrund unterschiedlicher Gitterdehnung kann zwischen der $\alpha$-Phase und der Hydridphase unterschieden werden. Diese Unterscheidung ist auch mit der oberflächensensitiven Methode STM möglich, da die verschiedenen Gitterabstände zu einer unterschiedlichen Ausdehnung in z-Richtung führen. Dieser Effekt ist in Abb. 4.4 dargestellt. An den Positionen, an denen sich das Hydrid bildet, steigt die Probenhöhe an. An den Rändern der Hydridausscheidungen ist eine Stufe der Höhe $\Delta z$ zu sehen. Das in rot eingezeichnete Höhenprofil entspricht den bei einer STM-Messung bestimmten Höheninformationen. Aus der Stufenhöhe $\Delta z$ und der ursprünglichen Filmdicke $h_{0}$ kann, wenn im Film keine Spannung in $z$-Richtung vorhanden ist, über die Gleichung (2.10) (s. S. 8) die Höhe der Hydridausscheidung bestimmt werden. Durch die Messung mit dem STM ist es somit möglich, die laterale Verteilung des Hydrids auf einer kleinen Skala, sowie die Entstehung und das Wachstum des Hydrids zu beobachten.

\subsubsection{Schichtdickenabhängigkeit der vertikalen lokalen Ausdehnung des Hydrids im Zweiphasengebiet}

In diesem Abschnitt soll die vertikale Ausdehnung des Hydrids untersucht werden. Es wird gemessen, wie groß die Dehnung $\Delta z$ ist, wenn sich das Hydrid bildet. Dabei soll insbesondere auf die Abhängigkeit von der Schichtdicke $h_{0}$ eingegangen werden. Es werden nachfolgend Schichten mit unterschiedlicher Dicke mit Wasserstoff beladen und die Veränderungen durch die Hydridbildung ausgemessen.

\subsubsection{Filmdicke $72 \mathrm{~nm}$}

Die laterale Verteilung von $\alpha$-Phase und Hydrid ist in Abb. 4.5 dargestellt. Dort ist ein $10 \times 10 \mu \mathrm{m}^{2}$ großes Übersichtsbild eines $72 \mathrm{~nm}$ dünnen Nb-Films zu sehen. Der Miscut des Substrates betrug $\vartheta<0.1^{\circ}$. Die Hydridbildung wurde bei einem Wasserstoffdruck von $5 \cdot 10^{-6}$ mbar beobachtet. Es sind deutlich zwei Bereiche mit unterschiedlicher Höhe zu erkennen. Im niedrigen bzw. dunkleren Bereich ist die Schicht noch in der $\alpha$-Phase. In den höheren Bereichen wurde in der Schicht schon das Hydrid gebildet. 


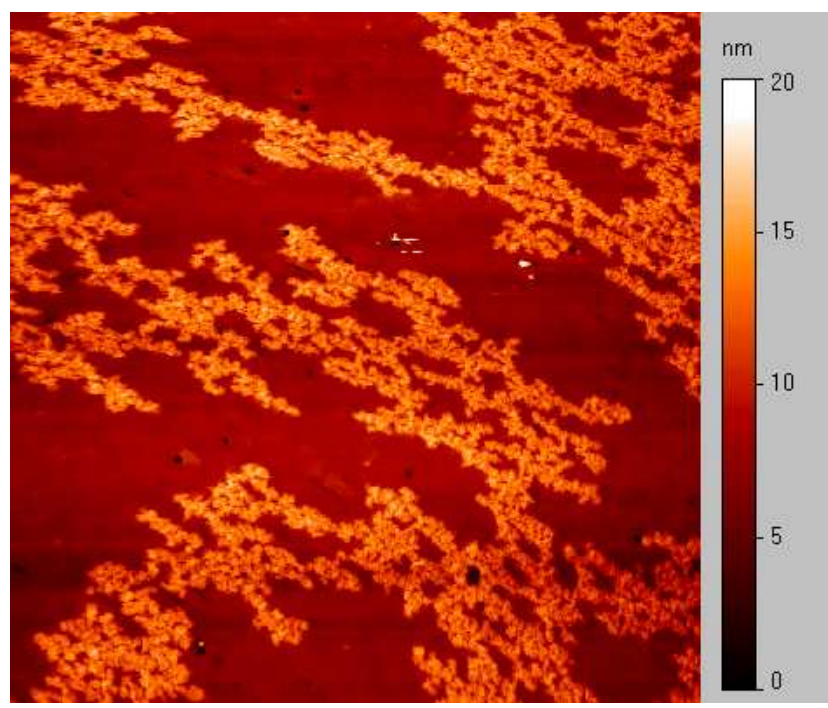

(a) STM-Bild, Größe: $10 \times 10 \mu \mathrm{m}^{2}$

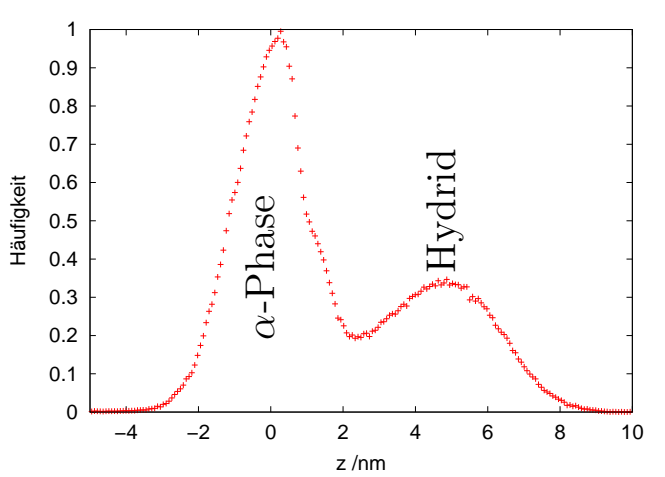

(b) Höhenverteilung

Abbildung 4.5: $72 \mathrm{~nm}$ dünner Nb-Film, beladen bei $5 \cdot 10^{-6}$ mbar Wasserstoffdruck. Die Schicht wurde auf einem Substrat mit einem Miscut kleiner als $0.1^{\circ}$ hergestellt. Es sind Bereiche mit $\alpha$-Phase (niedrig, dunkel) und Hydrid (hoch, hell) zu erkennen. Der Höhenunterschied von 4.7(5) nm kann gut in der Höhenverteilung (Teil (b)) abgelesen werden.

Diese Bereiche werden in der Höhenverteilung (Abb. 4.5(b)) durch zwei Maxima deutlich. Der Höhenunterschied zwischen $\alpha$-Phase und Hydrid kann so einfach abgelesen werden. Er beträgt für den $72 \mathrm{~nm}$ dünnen Film $\Delta z=4.7(5) \mathrm{nm}$.

\subsubsection{Filmdicke $80 \mathrm{~nm}$}

In Abb. 4.6 ist ein $2.5 \times 2.5 \mu^{2}$ großer Ausschnitt eines $80 \mathrm{~nm}$ dünnen Niobfilms (Substratmiscut $\vartheta<0.1^{\circ}$ ) dargestellt. Dieser Film wurde bei einem Druck von $9 \cdot 10^{-7}$ mbar beladen. Der Druck wurde über einen großen Zeitraum konstant gehalten, so dass das Hydrid nach der Bildung nur langsam gewachsen ist. In der STMAufnahme (vgl. Abb. 4.6(a)) sind zwei unterschiedliche Hydridausscheidungstypen zu sehen. Es treten kleine rundliche und langgestreckte große Formen auf. Auf die beiden Ausscheidungstypen wird in Abschnitt 4.2.2 eingegangen. Hier soll nun für die kleinen Ausscheidungen der Name T1 und für die großen der Name T2 vergeben werden. Der Höhenunterschied zur $\alpha$-Phase beträgt für den $80 \mathrm{~nm}$ dünnen Film bei den großen Ausscheidungen (Typ T2) 4.9(5) nm.

\subsubsection{Filmdicke $100 \mathrm{~nm}$}

Bei einem $100 \mathrm{~nm}$ dünnen Film auf einem Substrat mit einem Miscut kleiner als $0.1^{\circ}$ erkennt man in einem $10 \times 10 \mu \mathrm{m}^{2}$ Übersichtsbild (s. Abb. 4.7) auch wieder Berei- 


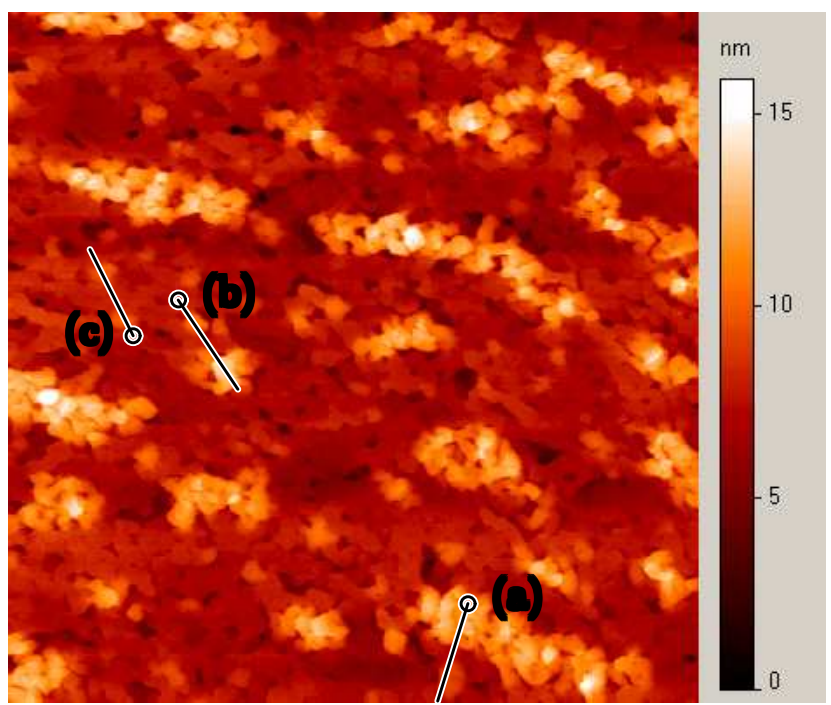

(a) STM-Bild, Größe: $2.5 \times 2.5 \mu \mathrm{m}^{2}$

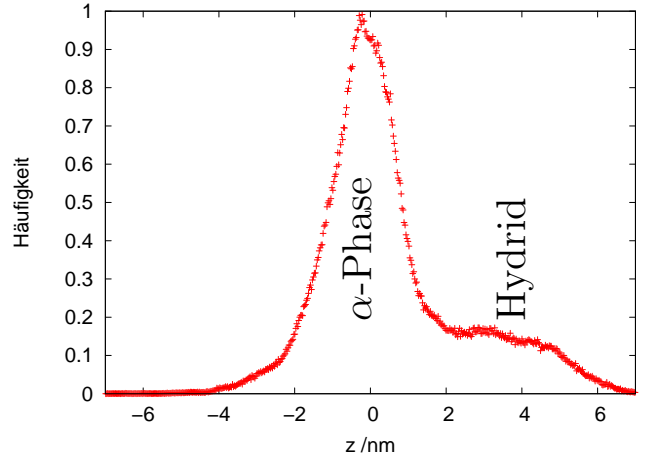

(b) Höhenverteilung

Abbildung 4.6: $80 \mathrm{~nm}$ dünner Nb-Film, beladen bei $9 \cdot 10^{-7}$ mbar Wasserstoffdruck (Substratmiscut $\vartheta<0.1^{\circ}$ ). Es sind Bereiche mit $\alpha$-Phase und Hydrid erkennbar. Es sind zwei unterschiedliche Hydridausscheidungstypen zu sehen. Es gibt kleine rundliche und große langgestreckte Ausscheidungen. Diese sind im Topographiebild (a) und in der Höhenverteilung (b) zu erkennen. Der Höhenunterschied zwischen der $\alpha$-Phase und den großen Ausscheidungen beträgt 4.9(5) nm. In Abb. 4.10 sind Höhenprofile der drei eingezeichneten Linien dargestellt.

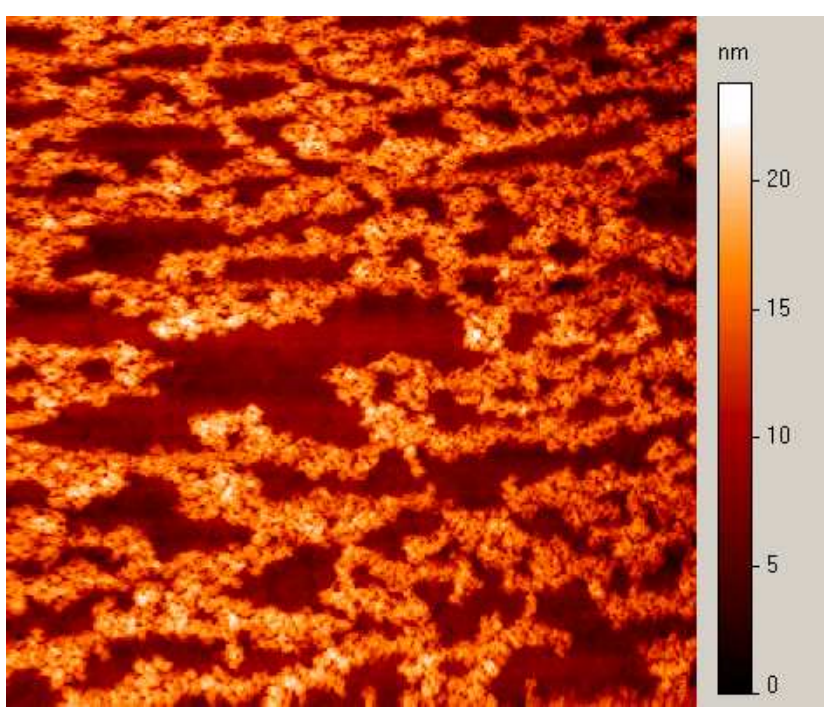

(a) STM-Bild, Größe: $10 \times 10 \mu \mathrm{m}^{2}$

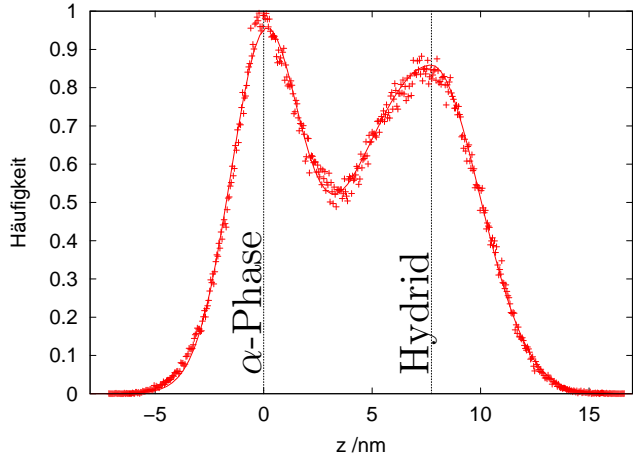

(b) Höhenverteilung

Abbildung 4.7: Koexistenz von Hydrid und $\alpha$-Phase bei einem $100 \mathrm{~nm}$ dünnen Niobfilm (Substratmiscut $\vartheta<0.1^{\circ}$ ). In Teil (a) ist die laterale Verteilung von Hydrid und $\alpha$ Phase dargestellt. Die Höhenverteilung des STM-Bildes ist in Teil (b) aufgetragen. Es sind deutlich zwei unterschiedliche Bereiche zu erkennen. 


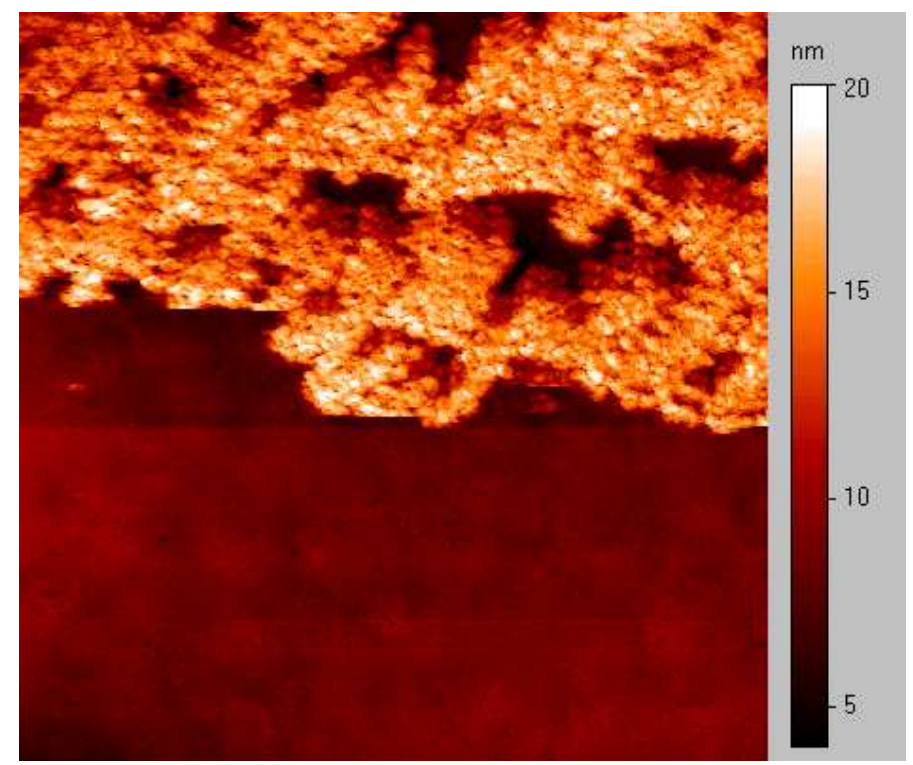

Abbildung 4.8: $120 \mathrm{~nm}$ dünner Niobfilm auf einem Substrat mit einem Miscut kleiner als $0.1^{\circ}$ bei einem Wasserstoffdruck von $8.2 \cdot 10^{-7}$ mbar beladen. Es sind $\alpha$-Phase und Hydrid zu sehen. Das Hydrid wächst vom oberen Bildteil langsam nach unten. Der Höhenunterschied von $\alpha$-Phase und Hydrid beträgt 8.3(5) nm. Größe: $7.5 \times 7.5 \mu \mathrm{m}^{2}$

che, die noch in der $\alpha$-Phase sind und andere Bereiche, innerhalb deren sich schon Hydrid gebildet hat. Der Film wurde bei einem Wasserstoffdruck von $1.7 \cdot 10^{-6} \mathrm{mbar}$ beladen, der Höhenunterschied zwischen $\alpha$-Phase und Hydrid beträgt 7.3(5) nm. In der Höhenverteilung in Abb. 4.7(b) sind die Maxima von $\alpha$-Phase und Hydrid unterschiedlich breit. Dies ist mit der unterschiedlichen Oberflächenunebenheit der beiden Bereiche zu erklären. Die Unebenheit der Oberfläche wird in Abschnitt 4.2.4 (S. 60) ausführlicher erläutert.

\subsubsection{Filmdicke $120 \mathrm{~nm}$}

Bei einem $120 \mathrm{~nm}$ dünnen Niobfilm auf einem Substrat mit einem Miscut $\vartheta<0.1^{\circ}$, dessen STM-Topographieabbildung in Abb. 4.8 gezeigt ist, beträgt der Höhenunterschied $\Delta z=8.3(5) \mathrm{nm}$. Der Film wurde bei einem Wasserstoffdruck von $8.2 \cdot 10^{-7} \mathrm{mbar}$ beladen. In dem $7.5 \times 7.5 \mu \mathrm{m}^{2}$ großen Bildausschnitt ist in der oberen Bildhälfte das Hydrid zu sehen, im unteren Bereich befindet sich die Schicht noch in der $\alpha$-Phase. An diesem Übergang kann der Höhenunterschied gut bestimmt werden. Das Hydrid wächst von der oberen Bildhälfte innerhalb einer Stunde über den gesamten Ausschnitt.

\subsubsection{Filmdicke $50 \mathrm{~nm}$}

Wie schon in Abb. 3.16 (s. S. 33) bei einem $50 \mathrm{~nm}$ dünnen Nb-Film (Substratmiscut $\vartheta<0.1^{\circ}$ ) erkennbar ist, tritt nach einiger Zeit bei einem Wasserstoffdruck von $1.4 \cdot 10^{-6}$ mbar eine Stufe der Höhe $\Delta z=3.5(5) \mathrm{nm}$ auf, die beim blauen und beim 
Tabelle 4.2: Höhenveränderungen zwischen Gebieten der $\alpha$-Phase und des Hydrids vom Typ T2 bei unterschiedlichen Schichtdicken.

\begin{tabular}{|c|c|c|c|}
\hline $\begin{array}{c}\text { Schichtdicke } \\
{[\mathrm{nm}]}\end{array}$ & $\begin{array}{c}\text { gemessene } \\
\text { Stufenhöhe }[\mathrm{nm}]\end{array}$ & $\begin{array}{c}\text { theoretische } \\
\text { Stufenhöhe }^{\dagger}[\mathrm{nm}]\end{array}$ & siehe \\
\hline 50 & $3.5(5)$ & 3.4 & Abb. 3.16 \\
\hline 72 & $4.7(5)$ & 4.9 & Abb. 4.5 \\
\hline 80 & $4.9(5)$ & 5.4 & Abb. 4.6 \\
\hline 100 & $7.3(5)$ & 6.8 & Abb. 4.7 \\
\hline 120 & $8.3(5)$ & 8.2 & Abb. 4.8 \\
\hline
\end{tabular}

magentafarbenen Höhenprofil besonders deutlich wird. Die dort auftretende Stufe entsteht, da das Hydrid der STM-Spitze in lateraler Richtung entgegenwächst. Außerdem ist beim Scannen in der Zeile $n$ an dieser Position noch kein Hydrid vorhanden. In der folgenden Zeile $n+1$ ist die Hydridausscheidung stark vergrößert. In dem resultierenden STM-Bild ist dann eine Stufe zu sehen. An dieser Stufe ist die Höhendifferenz zwischen $\alpha$-Phase und Hydrid leicht zu bestimmen.

\subsubsection{Alle Schichtdicken im Überblick}

Die Höhenunterschiede zwischen der $\alpha$-Phase und den Hydridausscheidungen vom Typ T2 für die unterschiedlichen Filmdicken sind in Tabelle 4.2 zusammengefasst. Die gemessenen Werte entsprechen den Höhenänderungen, die erwartet werden $\left[\mathrm{YSF}^{+} 96\right.$, $\mathrm{LFK}^{+}$99], wenn sich das Hydrid in $z$-Richtung durch die komplette Schicht bildet und keine Spannung in $z$-Richtung vorhanden ist. Bei einer Mischungslücke von $\Delta c=0.5$ wird nach Gl. (2.10) (s. S. 8) eine Ausdehnung der Schicht von $\Delta z=0.068 \cdot h_{0}$ erwartet. Diese theoretischen Stufenhöhen sind ebenfalls in der Tabelle angegeben. Sie stimmen sehr gut mit den experimentell gemessenen Höhenänderungen überein.

In Abb. 4.9 ist eine schematische Seitenansicht der Schicht dargestellt. Hier wird das Hydrid komplett vom Substrat bis an die Filmoberfläche gebildet. Wenn sich der Rand des Hydrids nicht wie in der Skizze dargestellt senkrecht zur Oberfläche bildet, wäre dennoch die maximale Ausdehnung durch die Schichtdicke nach Gl. (2.10) (s. S. 8) bestimmt. Der Übergang von der tieferen $\alpha$-Phase zum Hydrid wäre aber nicht so scharf wie in Abb. 4.9 eingezeichnet.

Die an der Oberfläche gemessene Ausdehnung $\Delta z$ ist proportional zu der in der Schicht gebildeten Hydridmenge (vgl. Gl. (2.10), S. 8). Da die Ausscheidungen vom Typ T2 in z-Richtung durch die gesamte Schicht reichen, gibt das Verhältnis der Flächen unter den Maxima von $\alpha$-Phase und Hydrid vom Typ T2 in den Höhenverteilungen (vgl. Abb. 4.5(b), 4.6(b) und 4.7(b), s. S. 52 - 53) den Anteil

\footnotetext{
${ }^{\dagger}$ Als theoretische Stufenhöhe ist die Umwandlung der kompletten Schicht nach Gl. (2.10) (s. S. 8) von der $\alpha$-Phase ins Hydrid angegeben. Als Breite der Mischungslücke wurde $\Delta c=0.5$ angenommen.
} 


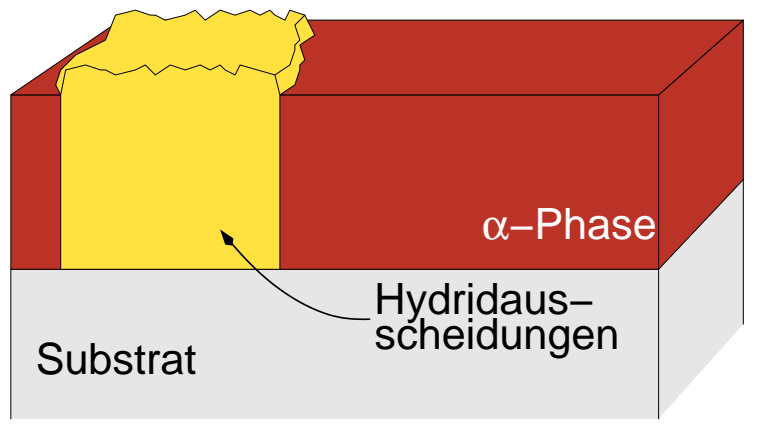

Abbildung 4.9: Schematische Verteilung des Hydrids in der Schicht. Die Hydridausscheidungen bilden sich in $z$-Richtung durch die komplette Schicht, in $x$ - und $y$-Richtung hat das Hydrid eine endliche Ausdehnung.

von $\alpha$-Phase und Hydridausscheidungen vom Typ T2 in der Schicht an. So wird in Abschnitt 4.3 die zeitliche Entwicklung des Hydridwachstums ausgemessen.

\subsubsection{Unterschiedliche Ausscheidungstypen}

Im STM-Bild der $80 \mathrm{~nm}$ dünnen Niobschicht aus Abb. 4.6 (s. S. 53) sind zwei unterschiedliche Ausscheidungstypen erkennbar. Die Unterschiede dieser beiden Typen sollen hier verdeutlicht werden. Dazu wird der laterale Übergang von der $\alpha$-Phase bis in das Hydrid betrachtet. In Abb. 4.10 sind drei Höhenprofile der $80 \mathrm{~nm}$ dünnen Niobschicht zu sehen, deren Positionen im STM-Bild auf Seite 53 in Abb. 4.6(a) durch Linien markiert sind. Der Nullpunkt des Profils ist durch einen Kreis markiert. In den Profilen (a)-(c) sind verschiedene Hydridausscheidungen zu erkennen. Wie auch schon im STM-Bild, sind in den Profilen zwei unterschiedliche Ausscheidungstypen zu sehen. Im STM-Bild sind zum einen kleine rundliche und zum anderen große langgestreckte Hydridausscheidungen erkennbar. Bei den kleineren Ausscheidungen ist ein flacher Höhenanstieg mit einem Anstiegswinkel von 2.7(3) ${ }^{\circ}$ in den Profilen erkennbar. Sie werden im Folgenden Typ T1 genannt. Die großen Ausscheidungen zeigen einen sehr steilen Höhenanstieg beim Übergang von der $\alpha$-Phase zum Hydrid. Sie werden Typ T2 genannt. Der Anstiegswinkel beträgt hier 16(2) ${ }^{\circ}$. Diese beiden unterschiedlichen Ausscheidungstypen sind auch bei einem $70 \mathrm{~nm}$ dünnen Niobfilm beobachtet worden (vgl. Abb. 4.11). In den Höhenprofilen in Abb. 4.11(b) und Abb. 4.11(c) ist der Höhenverlauf einiger Ausscheidungen vom Typ T1 und Typ T2 abgebildet. Bei den Ausscheidungen vom Typ T1 in Abb. 4.11(b) beträgt der Anstiegswinkel von der $\alpha$-Phase zur Hydridausscheidung 2.7(2) ${ }^{\circ}$. Für die Ausscheidungen vom Typ T2 beträgt der Anstiegswinkel 9(1) ${ }^{\circ}$ (vgl. Abb. 4.11(c)).

Ein weiterer Unterschied zwischen den beiden Typen T1 und T2 besteht im absoluten Höhenunterschied zur $\alpha$-Phase. Die Höhendifferenz bei einer $80 \mathrm{~nm}$ dünnen Niobschicht ist beim Typ T2 4.9(5) nm (vgl. Abschnitt 4.2.1.2, S. 52). Beim Typ T1 beträgt der Höhenunterschied bei gleicher Schichtdicke $\Delta z=2.1(3) \mathrm{nm}$. Diese unterschiedlichen Höhen der Ausscheidungstypen sind auch in der Höhenverteilung des 


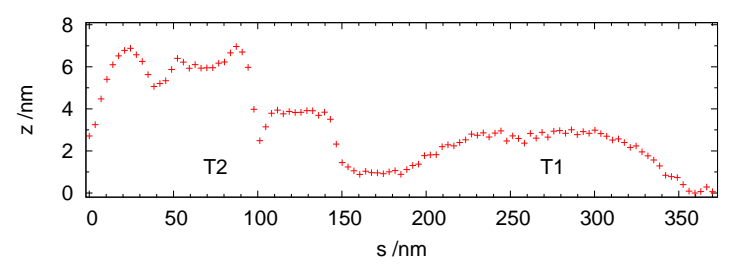

(a)

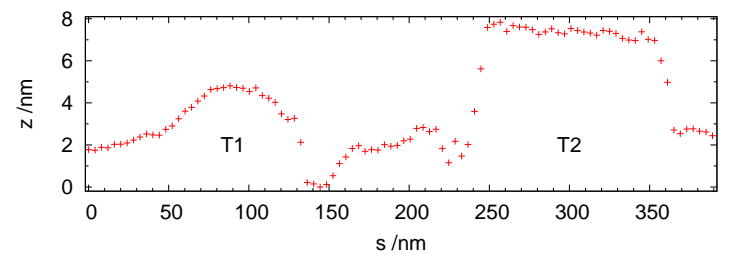

(b)

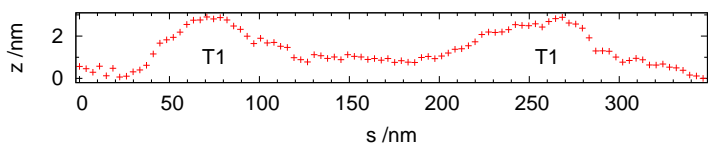

(c)

Abbildung 4.10: Höhenprofile entlang des Übergangs von der $\alpha$-Phase zum Hydrid bei einem $80 \mathrm{~nm}$ dünnen Nb-Film (vgl. Abb. 4.6, S. 53). Dort sind auch die Positionen der Profile markiert. Wie auch im STM-Bild sind im Höhenprofil zwei unterschiedliche Ausscheidungstypen zu erkennen. Beim Typ T1 findet die Höhenveränderung bei einem Anstiegswinkel von $2.7(3)^{\circ}$ langsam statt. Dagegen verändert sich die Höhe beim Übergang von der $\alpha$-Phase zum Typ T2 mit einem Anstiegswinkel von $16(2)^{\circ}$.

Tabelle 4.3: Größe der Ausscheidungen vom Typ T1. Für unterschiedliche Schichtdicken ist die Höhe, der Anstiegswinkel sowie der Durchmesser vom Ausscheidungstyp T1 zusammengestellt.

\begin{tabular}{|c|c|c|c|}
\hline $\begin{array}{c}\text { Schicht- } \\
\text { dicke }[\mathrm{nm}]\end{array}$ & $\begin{array}{c}\text { Ausscheidungs- } \\
\text { höhe }[\mathrm{nm}]\end{array}$ & $\begin{array}{c}\text { Anstiegs- } \\
\text { winkel }\left[{ }^{\circ}\right]\end{array}$ & $\begin{array}{c}\text { Radius } \\
{[\mathrm{nm}]}\end{array}$ \\
\hline 40 & $1.8(1)$ & $2.9(2)$ & $\leq 40(2)$ \\
\hline 70 & $1.8(1)$ & $2.7(2)$ & $\leq 36(3)$ \\
\hline 80 & $2.1(3)$ & $2.7(3)$ & $\leq 35(2)$ \\
\hline
\end{tabular}

STM-Bildes zu erkennen (vgl. Abb. 4.6(b), S. 53). Dort kann die Höhenverteilung gut mit einem Peak für die $\alpha$-Phase und zwei Gaußverteilungen für die unterschiedlichen Ausscheidungstypen angepasst werden. Der Höhenunterschied für den Typ T2 ist bei unterschiedlichen Schichtdicken in Tabelle 4.2 (s. S. 55) dokumentiert. Dort ist zu erkennen, dass dieser Ausscheidungstyp vertikal durch die gesamte Schicht wächst. Die Höhe der Ausscheidungen vom Typ T1 ist für unterschiedliche Schichtdicken in Tab. 4.3 aufgeführt. Es wird deutlich, dass die Höhendifferenz unabhängig von der Schichtdicke ist und ca. 1.9(3) nm beträgt. Der Anstiegswinkel beim Übergang von der $\alpha$-Phase zu Ausscheidungen vom Typ T1 liegt bei 2.8(3) ${ }^{\circ}$ und zeigt keine signifikante Schichtdickenabhängigkeit.

Die beiden Ausscheidungstypen unterscheiden sich auch in der Form. Die Ausscheidungen vom Typ T1 haben keine große laterale Ausdehnung und sind in der Aufsicht rund. Der Radius der an der Oberfläche sichtbaren Veränderung der Ausscheidungen vom Typ T1 zeigt eine geringe Abhängigkeit von der Schichtdicke. Mit abnehmen- 


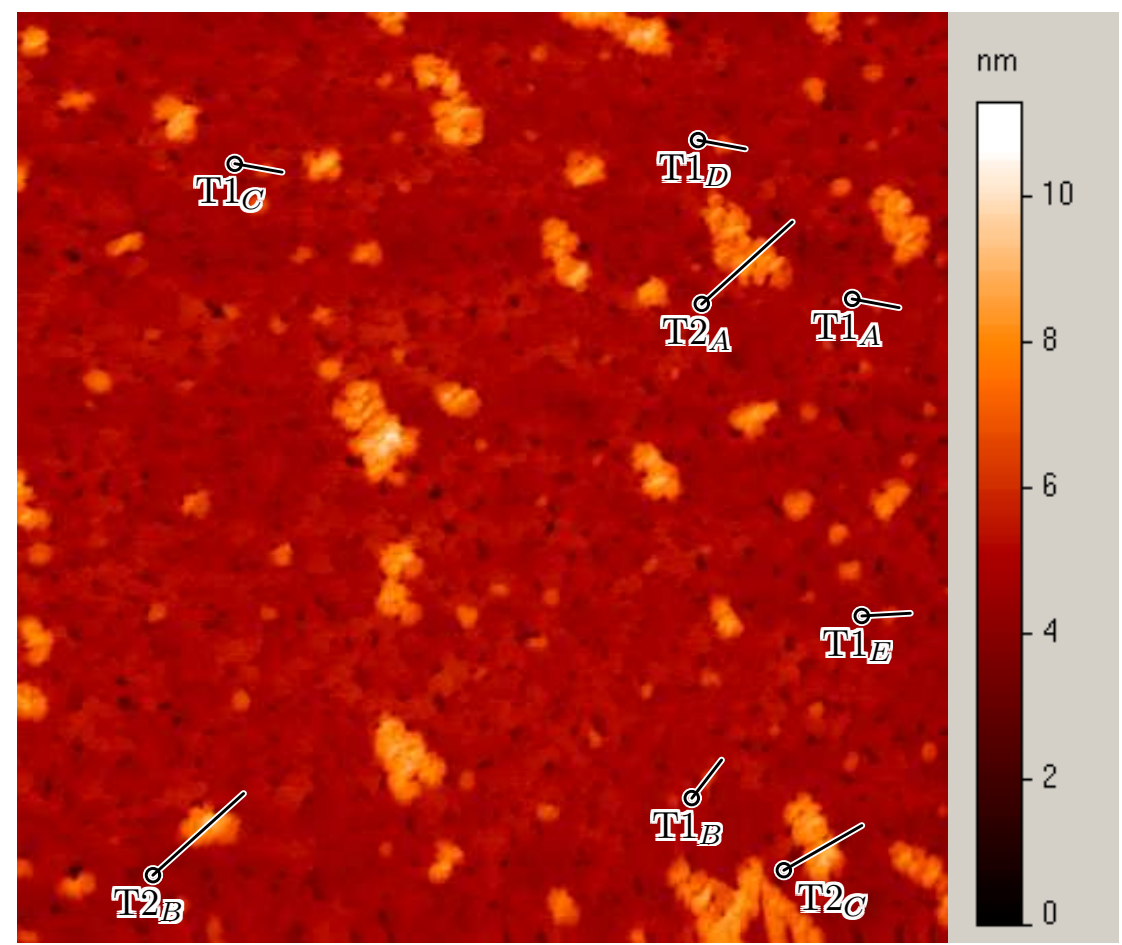

(a) STM-Bild, Größe $3.5 \times 3.5 \mu \mathrm{m}^{2}$

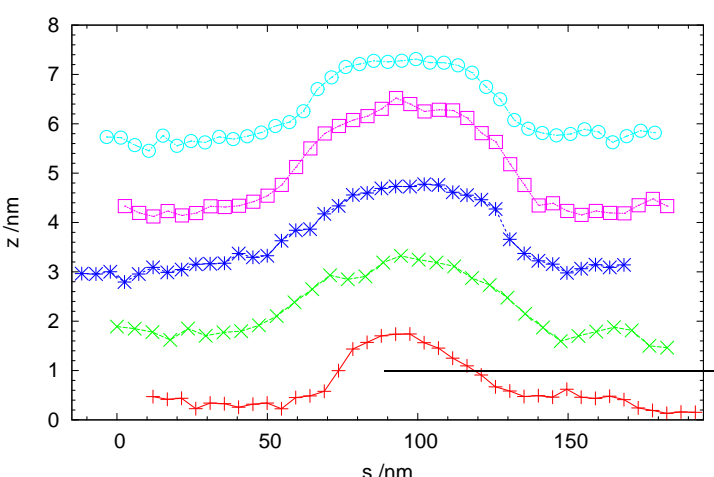

$\mathrm{T} 1_{A}+\mathrm{T} 1_{B} \cdots \mathrm{T} 1_{C} \cdots *-\mathrm{T}_{D}-\mathrm{T}_{E^{-0}}$

(b) Höhenprofile Typ T1

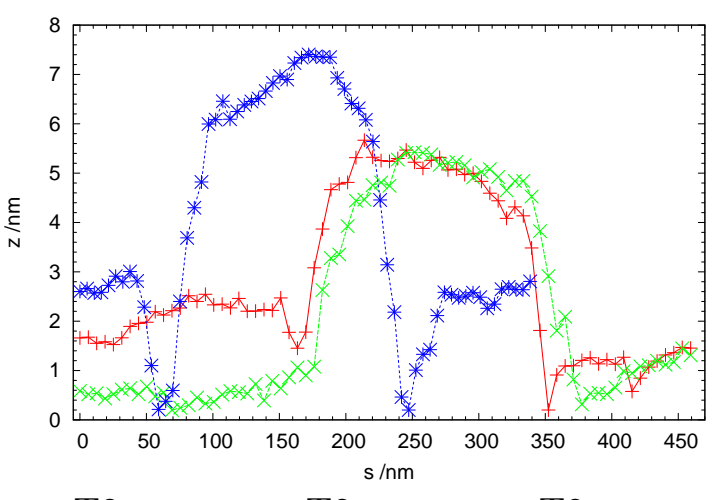

$$
\mathrm{T} 2_{A}+\mathrm{T} 2_{B}{ }^{-*} \quad \mathrm{~T} 2_{C} \cdots *
$$

(c) Höhenprofile Typ T2

Abbildung 4.11: Unterschiedliche Ausscheidungstypen eines $70 \mathrm{~nm}$ dünnen Niobfilms (Substratmiscut $\vartheta<0.2^{\circ}$ ). In (a) ist ein STM-Bild der Größe $3.5 \times 3.5 \mu \mathrm{m}^{2}$ abgebildet. Von diesem topographischen Bild sind an den eingezeichneten Linien Höhenprofile erstellt worden. Die Profile von den Ausscheidungstypen T1 sind in (b) dargestellt. Diese haben beim Übergang von der $\alpha$-Phase zur Ausscheidung einen Anstiegswinkel von $2.7(2)^{\circ}$. Ein Anstiegswinkel von $9(1)^{\circ}$ ist bei den in (c) zusammengestellten Profilen der Ausscheidungen vom Typ T2 gemessen worden. 
Tabelle 4.4: Zusammenstellung der Ausscheidungstypen für unterschiedliche Schichtdicken und Substratfehlschnitte. Für die einzelnen Schichten sind die beobachteten Ausscheidungstypen, die Anzahl der Ausscheidungen, sowie der Druck der Hydridbildung in mbar angegeben.

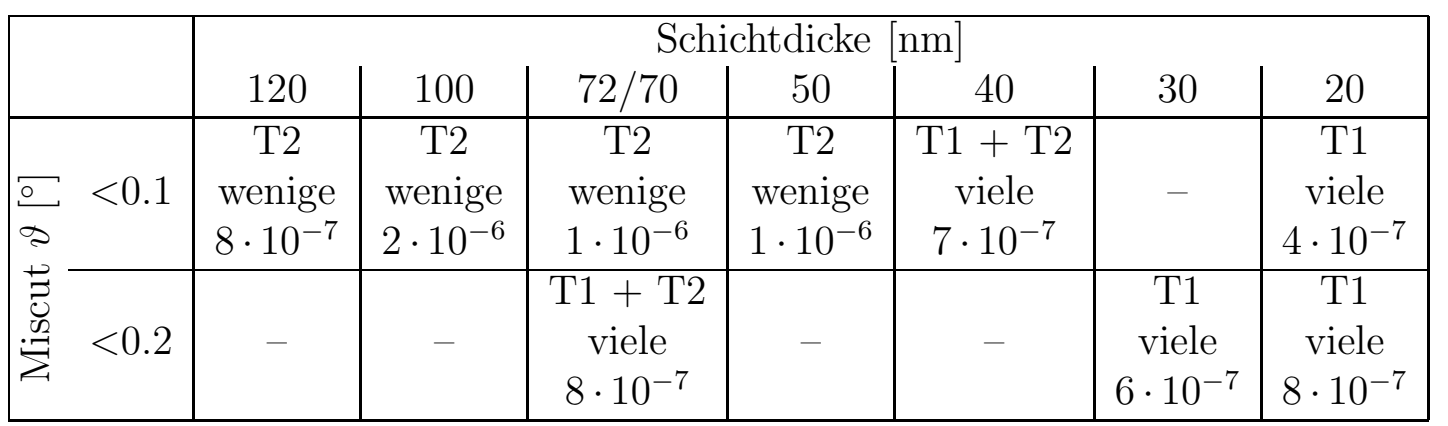

der Schichtdicke werden die größten noch vorhandenen Ausscheidungen vom Typ T1 größer. Bei einer Schichtdicke von $40 \mathrm{~nm}$ gibt es Ausscheidungen bis zu einem Radius von 40(2) nm. Im $70 \mathrm{~nm}$ dünnen Film sind keine T1 Ausscheidungen mit einem Radius größer als 36(3) nm vorhanden. Bei $80 \mathrm{~nm}$ Schichtdicke beträgt der maximale Radius 35(2) nm. Die anderen Ausscheidungen vom Typ T2 sind langgestreckt. Die Richtung der langen Achse hat eine bevorzugte Orientierung, die in Abschnitt 4.2.5.2 bestimmt wird. Die Größe der Ausscheidungen zeigt eine breite Streuung. Bei den großen Ausscheidungen vom Typ T2 in Abb. 4.6 (s. S. 53) hat die kurze Achse eine Ausdehnung von 100-200 nm. Die lange Achse reicht über mehrere $\mu \mathrm{m}$.

\subsubsection{Einflussparameter auf die Hydridbildung}

Nun soll der Einfluss von Substratmiscut und Schichtdicke auf die Existenz der beiden unterschiedlichen Ausscheidungstypen behandelt werden. Dazu werden die unterschiedlichen Schichten miteinander verglichen. Hierbei werden nur die Filme berücksichtigt, bei denen die Wasserstoffbeladung in vergleichbarer Form durchgeführt worden ist. Bei den Beladungen der hier behandelten Schichten ist der Druck schrittweise erhöht worden. Anschließend wurde der Druck für einen Zeitraum von bis zu $3 \mathrm{~h}$ konstant gehalten. Der nächste Beladungsschritt wurde dann bei einem höheren Wasserstoffdruck durchgeführt. Der eingestellte Druck unterscheidet sich bei den verschiedenen Schichten geringfügig. Die treibende Kraft der Hydridbildung unterscheidet sich somit minimal. Dieser geringfügige Einfluss der treibenden Kraft wird hier nicht näher untersucht.

In Tabelle 4.4 sind die Beobachtungen zu beiden Ausscheidungstypen bei verschiedenen Schichtdicken zusammengefasst. Es ist zu erkennen, dass mit der Abnahme der Schichtdicke ein Übergang von der Bildung des Typs T2 zum Typ T1 stattfindet. Bei dicken Filmen bilden sich wenige große ausgestreckte Ausscheidungen vom Typ T2. Bei geringerer Schichtdicke sind viele lateral kleine Hydridausscheidungen vom Typ T1 zu beobachten. Bei einem Substratmiscut kleiner als $0.1^{\circ}$ liegt der Übergang bei einer 


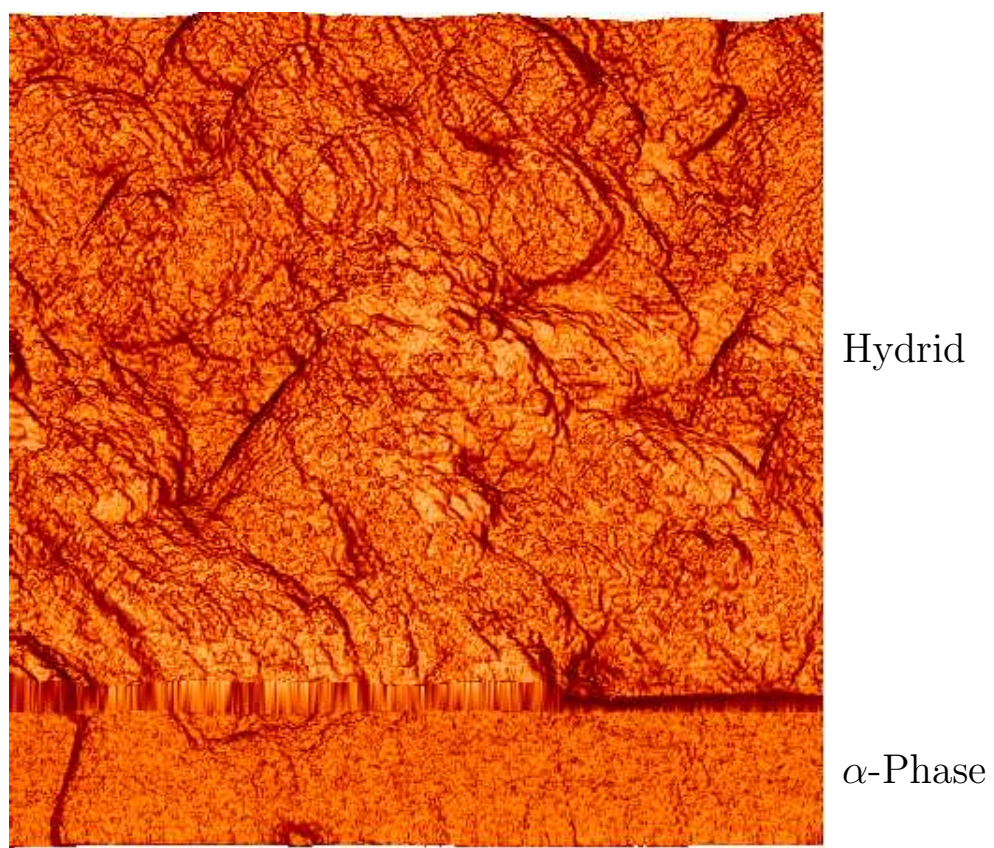

Abbildung 4.12: $100 \mathrm{~nm}$ dünner Nb-Film (Substratmiscut $\vartheta<0.1^{\circ}$ ) bei einem Wasserstoffdruck von $1.7 \cdot 10^{-6}$ mbar. 7.5 min nach dem Einlassen des Wasserstoffes trat ein Sprung in der Höhe von $7 \mathrm{~nm}$ auf. Unterhalb des Sprungs ist noch die $\alpha$-Phase zu sehen, darüber hat sich schon das Hydrid gebildet. Die Oberflächenunebenheit beider Gebiete unterscheidet sich um den Faktor 3.5. Bildgröße $500 \times 500 \mathrm{~nm}^{2}$.

Schichtdicke von $40 \mathrm{~nm}$. Wenn der Miscut des Substrates $\vartheta<0.2^{\circ}$ beträgt, wird der Übergang bei einer Schichtdicke von $70 \mathrm{~nm}$ beobachtet.

\subsubsection{Oberflächenmorphologie des Hydrids}

Nachdem dargestellt wurde, dass zwei unterschiedliche Hydridausscheidungstypen existieren, soll hier auf die Oberflächenmorphologie der beiden Typen eingegangen werden.

\subsubsection{Ausscheidungen vom Typ T1}

Bei Ausscheidungen vom Typ T1 sind keine Unterschiede in der Oberflächenmorphologie zwischen $\alpha$-Phase und Hydrid auszumachen. Die Höhe der Ausscheidung verändert sich, aber die Oberfläche der Ausscheidung gleicht der Oberfläche der $\alpha$-Phase. Der RMS-Wert der Ausscheidungen $(0.1 \mathrm{~nm})$ unterscheidet sich nicht signifikant von den Gebieten der $\alpha$-Phase. 


\subsubsection{Ausscheidungen vom Typ T2}

Bei den Ausscheidungen vom Typ T2 gibt es Unterschiede in der Oberfläche zur $\alpha$-Phase. Die Veränderung der Oberflächenunebenheit während der Entstehung von Typ T2-Hydridausscheidungen dokumentiert Abbildung 4.12. Es ist die gleiche $100 \mathrm{~nm}$ dünne Schicht wie in Abb. 4.7 (s. S. 53) dargestellt, jedoch zu einem früherem Zeitpunkt und mit $500 \times 500 \mathrm{~nm}^{2}$ in einem kleineren Bildausschnitt. Während der STMAufnahme hat die Umwandlung von der $\alpha$-Phase zum Hydrid stattgefunden. Im unteren, zuerst aufgenommenen Bildteil ist noch die $\alpha$-Phase zu erkennen. Dann tritt eine Stufe auf, da im oberen Bildausschnitt sehr schnell Hydrid gebildet wurde. Diese Umwandlung war so schnell, dass sie nicht von der STM-Messung aufgelöst werden konnte und nur eine Stufe in der Aufnahme zu sehen ist. Beim Scannen der Zeile $n$ wurde noch $\alpha$-Phase gemessen und bei der darauf folgenden Zeile $n+1$ wurde schon Hydrid beobachtet. Oberhalb der Stufe ist die Oberfläche des Hydrids zu sehen. Mit der Darstellung in Schattenmethode ist der Unterschied in der Oberflächenunebenheit gut zu erkennen. Die $\alpha$-Phase hat eine geringere Rauigkeit, diese ist bei der Herstellung entstanden. Durch die Bildung der Hydridausscheidungen vom Typ T2 vergrößert sich die Oberflächenunebenheit um den Faktor 3.5 auf einen RMS-Wert von $1.45 \mathrm{~nm}$ in dem Gebiet, in dem das Hydrid gebildet wurde. Dieser große Unterschied rührt daher, dass sich an der Hydridoberfläche sehr viele Stufen bilden. Auf diese Stufen und deren Ausrichtung wird in Abschnitt 4.2.6 noch einmal genauer eingegangen.

\subsubsection{Präferentielle Wachstumsrichtung des Hydrids}

\subsubsection{Ausscheidungen vom Typ T1}

Auf kurzer Reichweite sind die Ausscheidungen vom Typ T1 gegeneinander ausgerichtet. Dies ist in Abb. $4.13 \mathrm{zu}$ erkennen. Um die Ausscheidungen deutlicher zu erkennen, ist in Teil (a) ein Differenzbild von zwei nacheinander aufgenomenen STMTopographiemessungen einer $70 \mathrm{~nm}$ dünnen Niobschicht (Substratmiscut $\vartheta<0.2^{\circ}$, beladen bei $1 \cdot 10^{-6}$ mbar $\mathrm{H}_{2}$ ) dargestellt. Durch diese Differenzbildung werden die Veränderungen durch die Ausscheidungen besonders deutlich. Auf die Unterschiede durch die Drift während der STM-Messung soll hier nicht weiter eingegangen werden. Aus den Positionen der einzelnen Ausscheidungen lässt sich der Winkel von der Verbindungslinie zwischen zwei Ausscheidungen bestimmen. Eine Winkelverteilung aller auftretenden Orientierungen ist in Abb. 4.13(b) dargestellt. Bei dem in rot aufgetragenen Histogramm ist für jeden Messpunkt der Abstand $\xi$ mit den Faktor $1 / \xi^{3}$ gewichtet worden. Mit dieser Gewichtung des Abstands der Ausscheidungen ist eine Häufung entlang der $\langle 111\rangle$-Richtung vorhanden. Die Wichtung mit den Faktor $1 / \xi^{3}$ erklärt sich dadurch, dass in die Wechselwirkungsenergie von zwei Ausscheidungen der Abstand mit dieser Abhängigkeit eingeht. Diesen Zusammenhang von Wechselwirkungsenergie und Abstand ist von A. G. Khachaturyan und G. A. ShataLOV für ein isotropes Medium [KS69] und von H. YAmauchi und D. De FonTAINE für anisotropes Medium [YDF78] berechnet worden. Im Fall eines anisotropen 


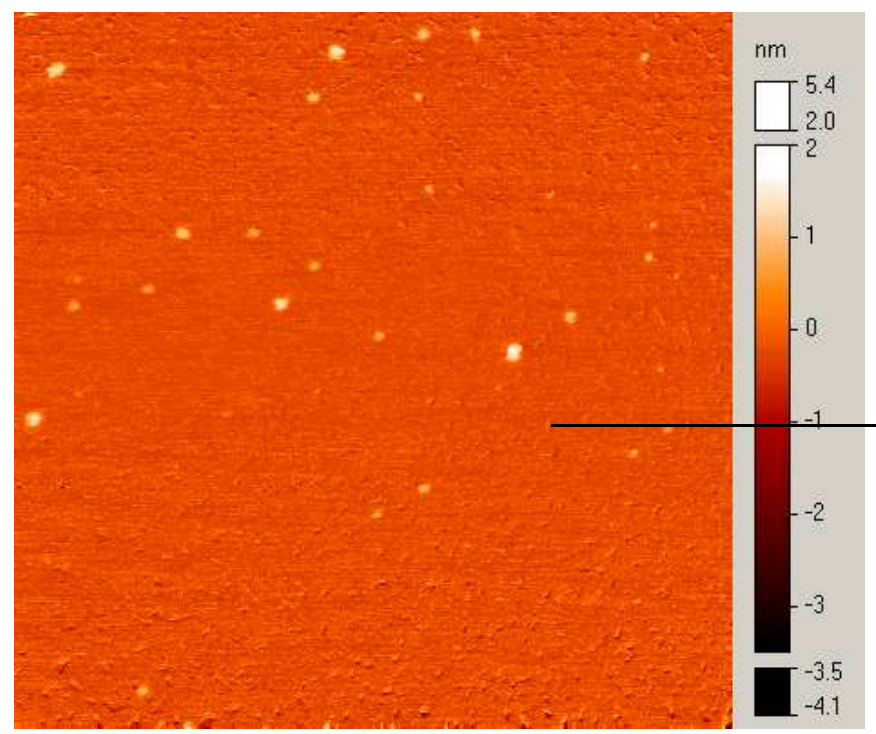

(a) STM-Differenzbild, Größe: $3.5 \times 3.5 \mu \mathrm{m}^{2}$

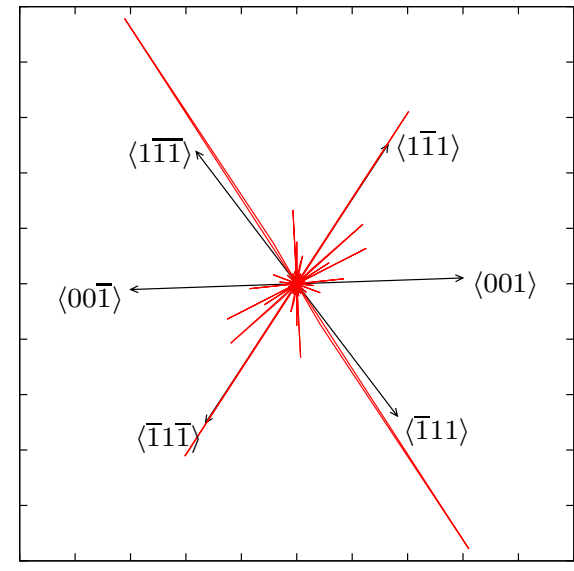

(b) gewichtete Winkelverteilung

Abbildung 4.13: Orientierung der Ausscheidungen vom Typ T1. Im Differenzbild von zwei STM-Aufnahmen (a), einer $70 \mathrm{~nm}$ dünnen Niobschicht (Miscut $\vartheta<0.2^{\circ}$ ), sind die Positionen der Ausscheidungen vom Typ T1 gut zu erkennen. Aus diesen Positionen ist dann der Winkel und der Abstand $\xi$ der Verbindungslinien zwischen zwei Ausscheidungen bestimmt worden. In der Verteilung (b) sind die Winkel mit den Faktor $\xi^{-3}$ gewichtet aufgetragen. Es ist deutlich eine bevorzugte Orientierung entlang der $\langle 111\rangle$-Richtung vorhanden.

Mediums zeigt die Wechselwirkungsenergie eine Richtungsabhängigkeit. Diese Richtungsabhängigkeit verursacht eine bevorzugte Ausrichtung in die elastisch weichen Richtungen. Diese Orientierung entlang der elastisch weichen $\langle 111\rangle$-Richtungen (vgl. Abb. 2.8(b), S. 12) ist in Abb. 4.13(b) deutlich zu erkennen.

\subsubsection{Ausscheidungen vom Typ T2}

In den Übersichtsbildern der Abbildungen 4.5, 4.6 und 4.7 (s. S. 52 - 53) wird dokumentiert, dass sich das langgestreckte Hydrid vom Ausscheidungstyp T2 in zwei bevorzugten Richtungen bildet. Um diese Richtungen kristallographisch zu bestimmen, muss zunächst die Orientierung des Films bestimmt werden. Hierzu werden an der Oberfläche der $\alpha$-Phase die Richtungen der Terrassenstufen bestimmt. Diese Terrassenstufen bilden sich aus energetischen Gründen bevorzugt in niedrig indizierten Kristallrichtungen aus. Im $\langle 110\rangle$ zur Filmoberfläche orientiertem bcc-Kristall sind die $\langle 001\rangle$-, $\langle\overline{1} 11\rangle$ - und $\langle 1 \overline{1} 1\rangle$-Richtungen bevorzugte Stufenkanten. In Abb. 4.14(a) sind Terrassenstufen in einem STM-Bild markiert. Die Richtungen dieser Stufen sind in einer in Polarkoordinaten erzeugten Verteilung aufgetragen (s. Abb. 4.14(b)). Bei der gegebenen Winkelbeziehung zwischen den drei bevorzugten Richtungen lässt sich die Orientierung dieser Schicht eindeutig bestimmen. Aufgrund dieser Filmorientierung 


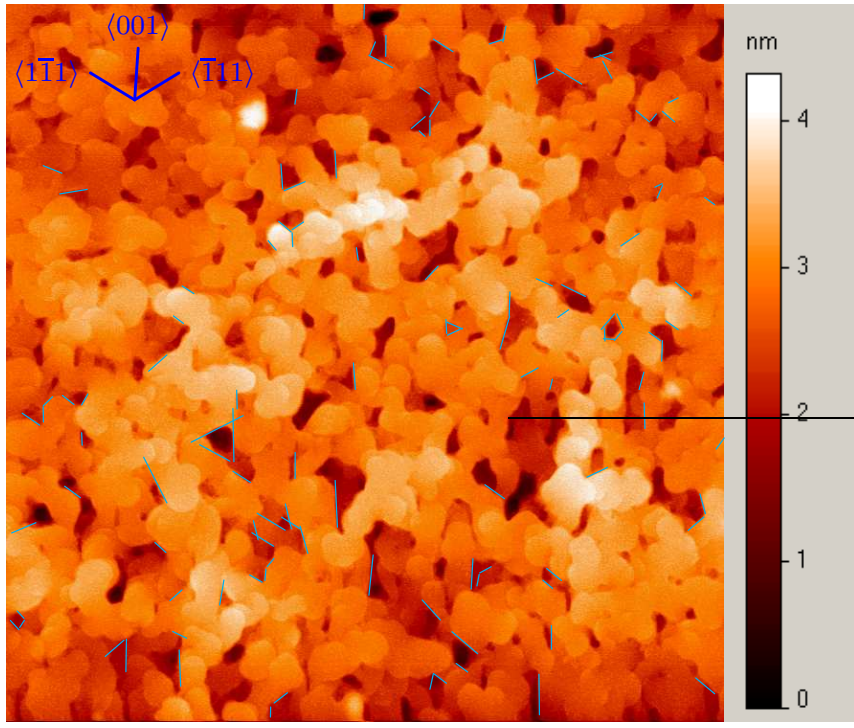

(a) STM-Bild, Größe: $1.5 \times 1.5 \mu \mathrm{m}^{2}$

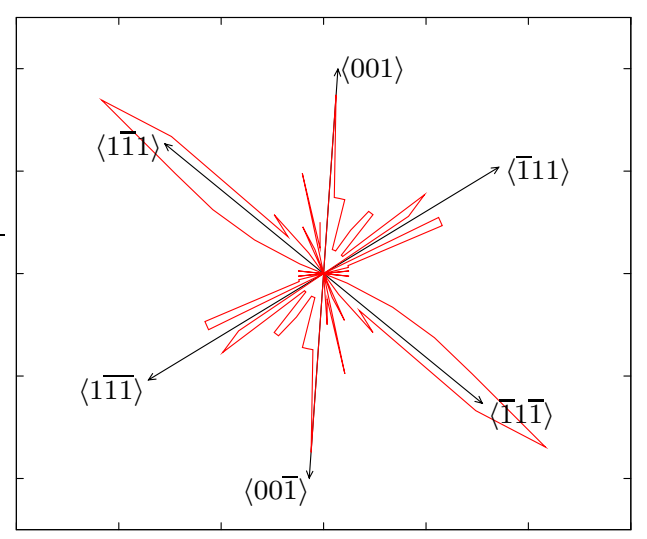

(b) Winkelverteilung

Abbildung 4.14: Bestimmung der Filmorientierung. Im STM-Bild eines $100 \mathrm{~nm}$ dünnen Films (a) sind gerade Terrassenstufen zu erkennen. Die Richtungen der Terrassenstufen sind in der Häufigkeitsverteilung (b) aufgetragen. Die auftretenden Maxima lassen sich anhand der Winkel zwischen den $\langle 001\rangle,\langle\overline{1} 11\rangle$ und $\langle 1 \overline{1} 1\rangle$ Richtungen eindeutig einer Richtung zuordnen.

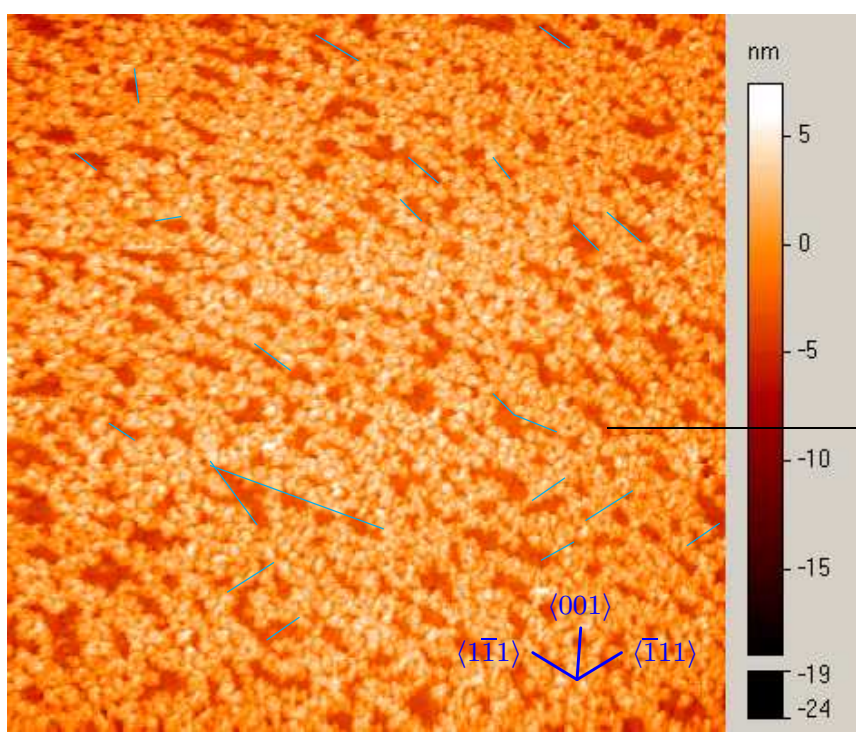

(a) STM-Bild, Größe:7.5 × $7.5 \mu \mathrm{m}^{2}$

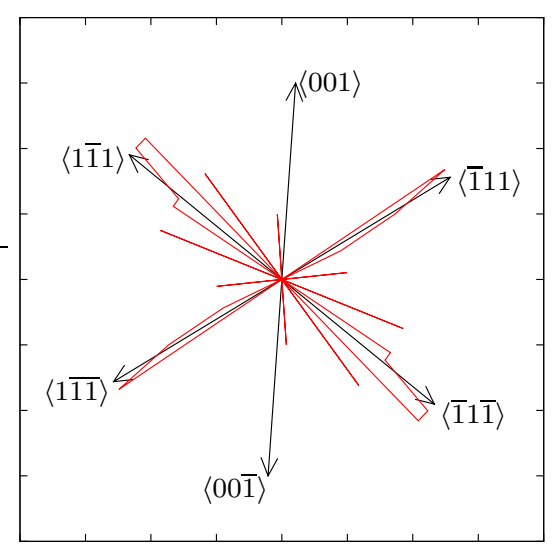

(b) Winkelverteilung

Abbildung 4.15: Bestimmung der Vorzugsrichtung der Hydridbildung. Die Filmorientierung dieser $100 \mathrm{~nm}$ dünnen Schicht wurde in Abb. 4.14 bestimmt. Im STM-Bild (a) wurden die bevorzugten Richtungen markiert und eine Winkelverteilung (b) erstellt. Das Hydrid bildet sich bevorzugt entlang der $\langle 111\rangle$-Richtungen der $\alpha$-Phase aus. 
kann nun die bevorzugte Orientierung bei der Hydridbildung bestimmt werden. Dazu sind in Abb. 4.15 die Richtungen, in denen das Hydrid orientiert ist, markiert. Mit den so erhaltenen Richtungen wurde eine Winkelverteilung erzeugt (vgl. Abb. 4.15(b)). In dieser Verteilung sind die schon bekannten Richtungen der Schicht eingezeichnet und es ist zu erkennen, dass das Hydrid entlang der $\langle 111\rangle$-Richtungen, der ursprünglichen $\alpha$-Phase, bevorzugt wächst. In Abschnitt 2.2.4 (s. S. 9) wurde auf die mechanischen Spannungen in mit Wasserstoff beladenen dünnen Schichten eingegangen. Dort wurde auch die Richtungsabhängigkeit des Elastizitätsmoduls angegeben. In Abb. 2.8(b) (s. S. 12) ist der E-Modul in der (110)-Ebene aufgetragen. Die $\langle 111\rangle$-Richtungen sind die elastisch weichen Richtungen im Niob. Das Hydrid wächst also bevorzugt entlang der elastisch weichen $\langle 111\rangle$-Richtungen des Niobs.

\subsubsection{Versetzungen im Hydrid und Bestimmung des Gleitsystems}

Nach der Hydridbildung ist die Oberflächenunebenheit deutlich erhöht (vgl. Abb. 4.12, S. 60). In dieser Abbildung sind im oberen Teil, in dem sich Hydridausscheidungen vom Typ T2 gebildet haben, Stufen zu erkennen. Diese Stufen haben bevorzugte Richtungen. Die Stufen entstehen durch Versetzungen, die an die Oberfläche wandern (vgl. Abb. 2.9(b), S. 14). In Abb. 4.16(a) ist die Schicht noch einmal dargestellt und die bevorzugten Richtungen der Stufen sind hier gut erkennbar. Da es in bcc-Gittern möglich ist, dass Versetzungen entlang verschiedener $\langle 111\rangle$-Richtungen quergleiten [Tei, WW92], sind die Richtungen der Stufen nicht über große Strecken erhalten. Es sind nur kurze Gleitstufen sichtbar. Dies ist anders als es von D. G. NAGENGAST et.al. $\left[\mathrm{NKvG}^{+}\right.$99] bei hcp-Yttrium beobachtet wurde, als bei der Wasserstoffbeladung lang ausgestreckte Stufen entstanden sind. Ebenso wurden bei der Hydridbildung von hcp-Gadolinium von A. PUnd et.al. langgestreckte Stufen beobachtet $\left[\mathrm{PGB}^{+} 00\right]$. Die hier beim Niob beobachteten kurzen Gleitstufen haben zwei bevorzugte Ausrichtungen, was in der Winkelverteilung der Stufen in Abb. 4.16(b) erkennbar ist. Dort ist über verschiedene STM-Bilder summiert die Häufigkeit der verschiedenen Ausrichtungen der Gleitstufen aufgetragen. Der Winkel zwischen den beiden bevorzugten Richtungen beträgt $98(2)^{\circ}$. In bcc-Metallen gibt es die möglichen Gleitebenen $\{110\}$, $\{112\}$ und $\{123\}$ [Rob00]. Die Stufen, die an der Oberfläche auftreten können, sind dadurch bestimmt, dass die Schnittlinie einer Gleitebene mit der Filmoberfläche errechnet wird. Bei zwei unterschiedlichen Gleitebenen ergeben sich zwei Schnittlinien mit einem Winkel zueinander. Alle möglichen Ebenenkombinationen sind in Tabelle 4.5 mit den resultierenden Winkeln angegeben. Der Winkel von $100^{\circ}$, der einmal auftaucht, passt zum gemessenen Winkel. Damit erhält man bei einer (110)-Oberfläche die Gleitebenen $(1 \overline{2} 1)$ und (011) und die Richtungen $\langle 1 \overline{1} 1\rangle$ und $\langle\overline{1} 13\rangle$ für die Gleitstufen. Das Gleitsystem ist somit bestimmt. 


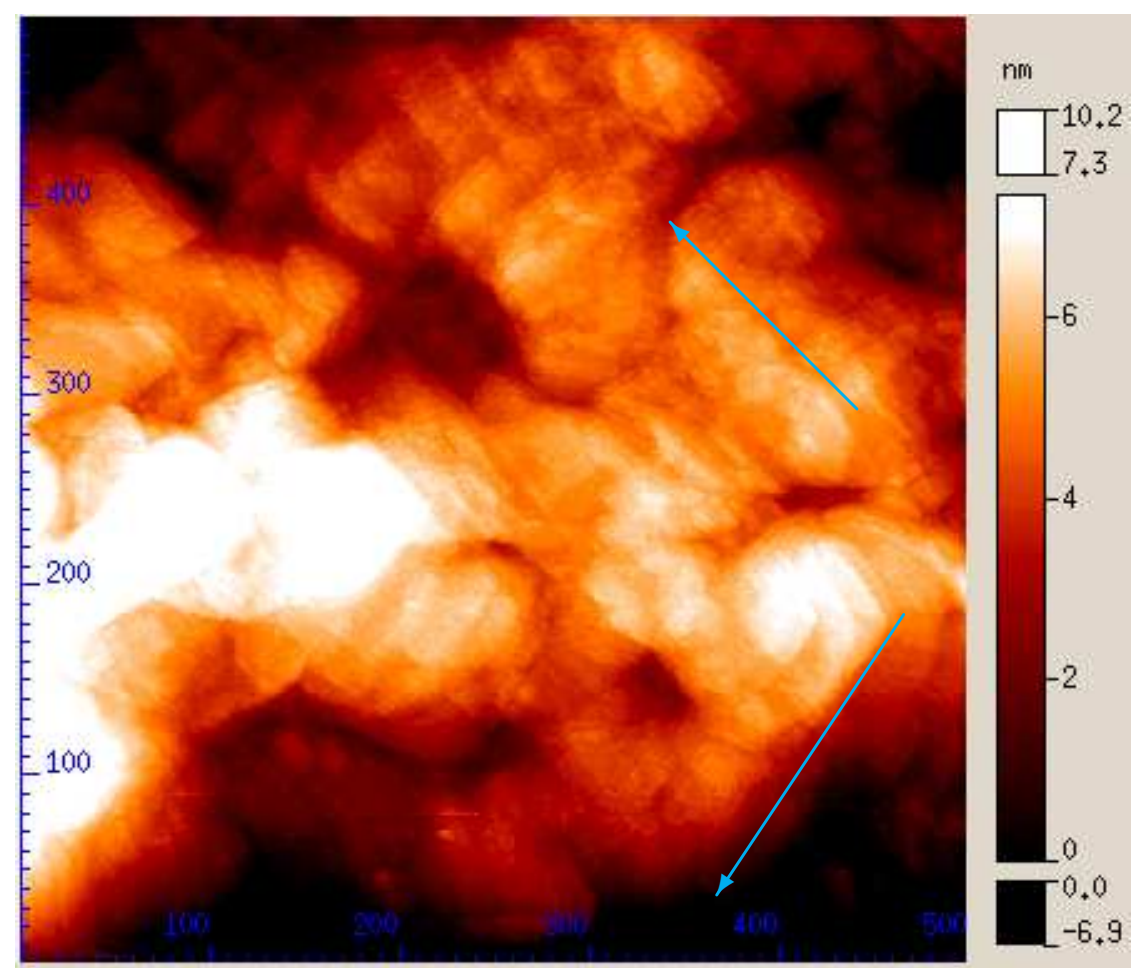

(a) STM-Bild, Größe $500 \times 500 \mathrm{~nm}^{2}$

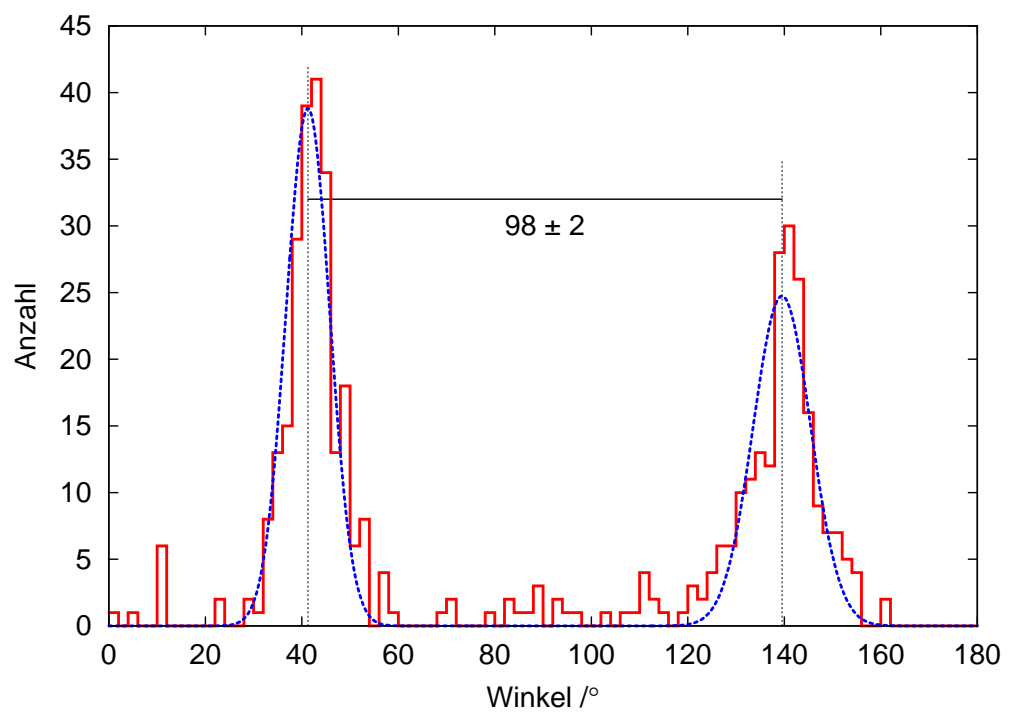

(b) Häufigkeitsverteilung der Stufen

Abbildung 4.16: Gleitstufen bei der Hydridbildung bei einer $100 \mathrm{~nm}$ Nb-Schicht. Im STMBild (a) sind deutlich zwei bevorzugte Richtungen - markiert durch zwei Pfeile - zu sehen. In der Winkelverteilung (b) wurden unterschiedliche STM-Bilder summiert. Der Winkel zwischen den beiden Richtungen beträgt $98(2)^{\circ}$. 
Tabelle 4.5: Winkel zwischen den Schnittlinien unterschiedlicher Gleitebenen an der Oberfläche im bcc-Gitter. Bei gegebener (110)-Oberfläche wurden für die ausgewählten Ebenensysteme alle möglichen Schnittlinien von Oberfläche und Ebene bestimmt. So kann dann der Winkel zwischen dieser Schnittlinie und einer Schnittlinie aus einer anderen Ebene berechnet werden.

\begin{tabular}{|c|c|c|c|}
\hline Oberfläche & \multicolumn{2}{|c|}{ Ebenen } & Winkel $\left[^{\circ}\right]$ \\
\hline$(110)$ & $\{011\}$ & $\{101\}$ & $54.7,70.5,109.5,125.3$ \\
\hline$(110)$ & $\{011\}$ & $\{123\}$ & $\begin{array}{l}15.8,22.0,38.9,48.5,54.7,70.5,76.7,103.3,109.5, \\
125.3,131.5,141.1,158.0,164.2\end{array}$ \\
\hline$(110)$ & $\{011\}$ & $\{112\}$ & $\begin{array}{l}25.2,29.5,35.2,54.7,70.5,80.0, \mathbf{1 0 0 . 0}, 109.5 \\
144.8,150.5,154.8\end{array}$ \\
\hline
\end{tabular}

\subsubsection{Randbereich zwischen $\alpha$-Phase und Hydrid}

Wenn das Hydrid gebildet wird, treten zwischen den Gitterabständen von $\alpha$-Phase und Ausscheidungen große Unterschiede auf. Diese Unterschiede erzeugen mechanische Spannungen zwischen den Ausscheidungen und der Matrix. Bei den großen Ausscheidungen des Typs T2 treten plastische Verformungen auf, wie dies an der Bildung von Gleitstufen in den Hydridgebieten zu erkennen ist (Abb. 4.16). Ob diese plastische Verformung im Hydrid auch Auswirkungen auf die $\alpha$-Phase hat, soll nun betrachtet werden.

\subsubsection{Ausscheidungen vom Typ T1}

Im Grenzbereich zwischen Ausscheidungen und $\alpha$-Phase steigt die Höhe bei verschiedenen Schichtdicken unter einem Winkel von $2.8(3)^{\circ}$ an (vgl. Tabelle 4.3, S. 57). Eine Veränderung in den Gebieten um die Ausscheidung ist nicht festgestellt worden. Folglich verursachen die Typ T1-Ausscheidungen keine Veränderungen an der Oberfläche der angrenzenden $\alpha$-Phase.

\subsubsection{Ausscheidungen vom Typ T2}

In Abb. 4.17 ist die Koexistenz von $\alpha$-Phase und einer Hydridausscheidung vom Typ T2 bei einer $50 \mathrm{~nm}$ Niobschicht dargestellt. Der dreidimensionale Ausschnitt hat eine Größe von $500 \times 500 \mathrm{~nm}^{2}$, die Höhenskala überdeckt einen Bereich von 0 bis $8.46 \mathrm{~nm}$. Die Farbkodierung wurde so gewählt, dass der Übergang von der $\alpha$-Phase zum Hydrid gut sichtbar ist. Der Farbübergang im rosafarbenen Bereich gibt die unterschiedlichen Höheninformationen der Gebiete wieder, in denen sich die Schicht noch in der $\alpha$-Phase befindet. Die Höheninformationen der Hydridgebiete werden durch unterschiedliche Gelbtöne dargestellt. Im rosafarbenen Bereich ist die Oberfläche im Wesentlichen glatt. Es sind monoatomare Terrassenstufen zu erkennen, die bei der Schichtherstellung entstanden sind. Eine geringe Oberflächenrauigkeit wird durch PdCluster an der Oberfläche hervorgerufen. Zusätzlich sind noch Kanten sichtbar, die 


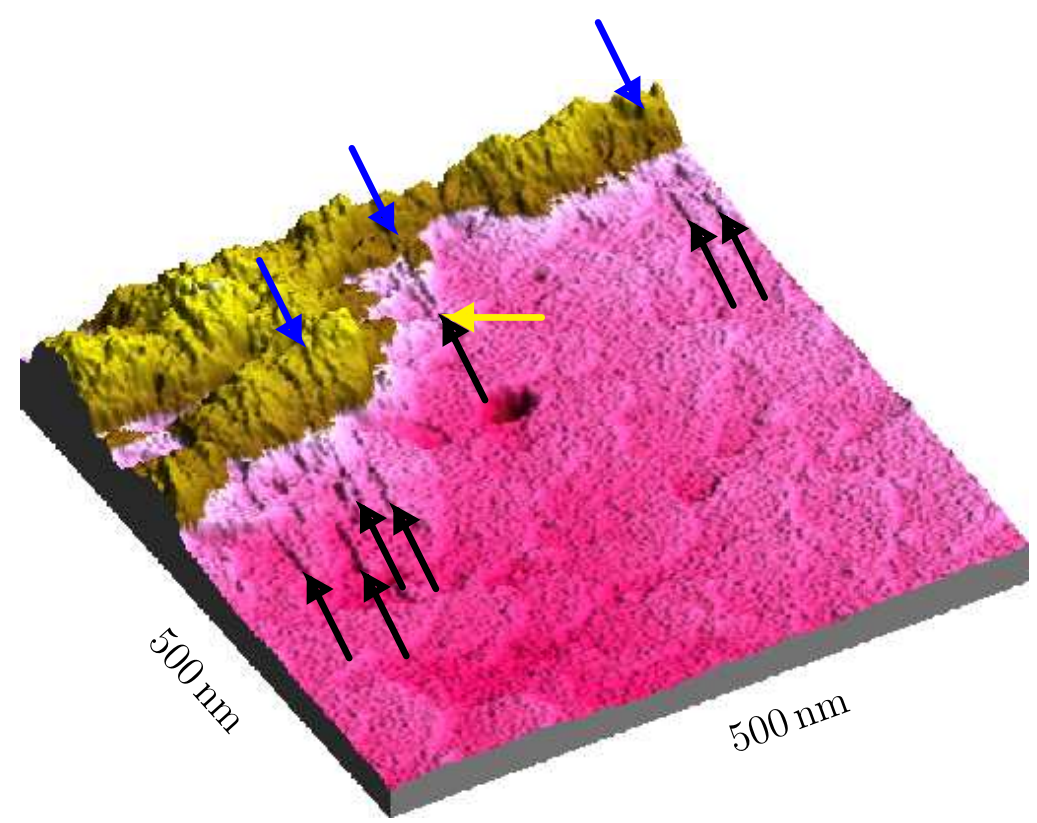

(a) dreidimensional

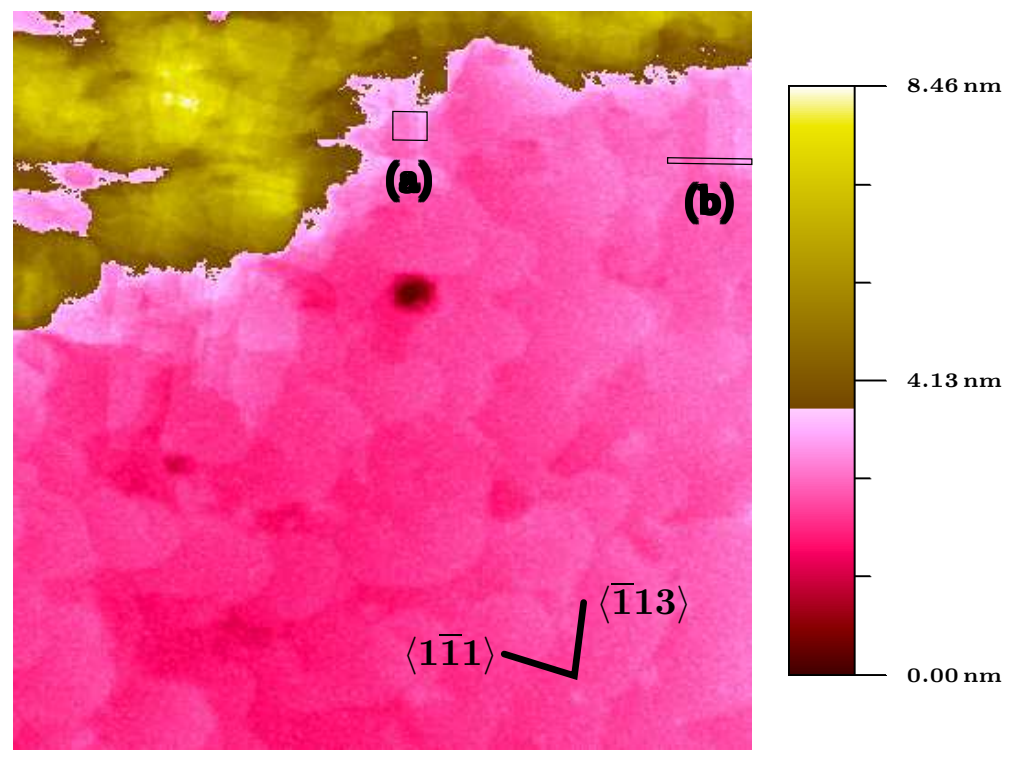

(b) zweidimensional

Abbildung 4.17: Sichtbare Gleitstufen in der $\alpha$-Phase und im Hydrid bei einem $50 \mathrm{~nm}$ dünnen Nb-Film. Bei der Farbskalierung ist darauf geachtet worden, dass der Kontrast zwischen der $\alpha$-Phase (rosa) und dem Hydrid (gelb) groß ist. In Teil (a) ist bei der dreidimensionalen Darstellung die Position der Lichtquelle so gewählt worden, dass die Gleitstufen sichtbar werden. Die Gleitstufen in der $\alpha$-Phase, von denen einige mit schwarzen Pfeilen markiert sind, können bis in das Hydrid verlängert werden (mit blauen Pfeilen hervorgehoben). Von den in (b) mit Rechtecken markierten Bereichen sind Höhenprofile erstellt worden (s. Abb. 4.18). Die eingezeichneten Richtungen wurden anhand der Gleitstufenorientierung bestimmt (s. Abb. 4.19). Für das Erstellen der Bilder wurde das Programm WSxM verwendet [WSx]. 


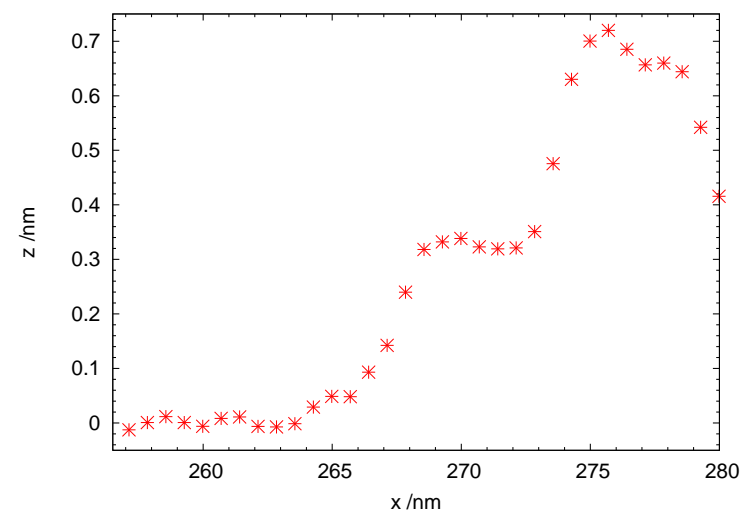

(a) Mittelung über 20 Linien

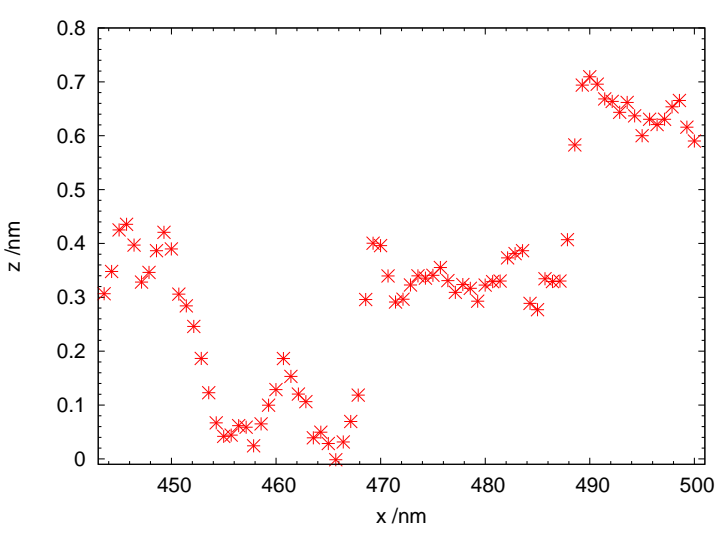

(b) Mittelung über 4 Linien

Abbildung 4.18: Höhenprofile über Gleitstufen aus Abb. 4.17 eines $50 \mathrm{~nm}$ dünnen Niobfilms. Aufgetragen ist die über mehrere $y$-Zeilen gemittelte Höhe $z$ in Abhängigkeit von der $x$-Koordinate. In beiden Profilen sind Stufen zu sehen. Die Stufenhöhe beträgt jeweils $0.32(3) \mathrm{nm}$, was der Gitterkonstanten von Niob entspricht.

nach der Hydridbildung neu entstanden sind. Hierbei handelt es sich um Gleitstufen, die sich bis in den Bereich des Hydrids erstrecken. Einige von den in der $\alpha$-Phase sichtbaren Gleitstufen sind durch schwarze Pfeile markiert. Diese Stufen lassen sich bis in das Hydrid verlängern. Eine Auswahl der Fortführungen der Gleitstufen in das Hydrid hinein sind mit blauen Pfeilen verdeutlicht. Alle Gleitstufen, die in der $\alpha$-Phase zu erkennen sind, haben eine Fortführung der Stufe im Hydrid. Mit einem gelben Pfeil ist zusätzlich noch eine Gleitstufe markiert, deren Ausrichtung sich von den anderen Gleitstufen unterscheidet. Höhenprofile, die senkrecht zu den Stufen erstellt wurden, sind in Abb. 4.18 zu sehen. Dort sind zwei Höhenprofile aus unterschiedlichen Bildpositionen aufgetragen. Es ist über mehrere Zeilen in $y$-Richtung gemittelt worden. Die $x$-Koordinaten sind als $x$-Achse gewählt worden. Die Bereiche, in denen die Höhenprofile erstellt worden sind, sind in Abb. 4.17(b) durch Rechtecke markiert. In den Profilen sind mehrere Stufen zu sehen. Die Stufenhöhe beträgt 0.32(3) nm. Dies entspricht dem Gitterparameter für Niob von $a=0.33 \mathrm{~nm}$ [HF76]. Das legt den Schluss nahe, dass die Stufen als monoatomare Gleitstufen anzusehen sind, die beim Abbau der mechanischen Spannung zwischen Hydrid und $\alpha$-Phase entstehen.

In Abschnitt 4.2 .6 (s. S. 64) ist anhand der Orientierung der Gleitstufen das Gleitsystem bestimmt worden. Mit dieser Information kann die Orientierung der Schicht in Abb. 4.17 bestimmt werden. Dazu ist in Abb. 4.19 eine Häufigkeitsverteilung der Stufenrichtungen aufgetragen. Es treten zwei bevorzugte Richtungen auf, die sich durch die Winkelbeziehung untereinander eindeutig den $\langle 1 \overline{1} 1\rangle$ - und $\langle\overline{1} 13\rangle$-Richtungen zuordnen lassen. So sind die beiden Richtungen bestimmt worden, die in Abb 4.17(b) eingezeichnet sind. Die Gleitstufen, die in der $\alpha$-Phase sichtbar sind, treten hauptsächlich entlang der $\langle\overline{1} 13\rangle$-Richtung auf. In dieser Richtung reichen die Stufen bis zu $120 \mathrm{~nm}$ 


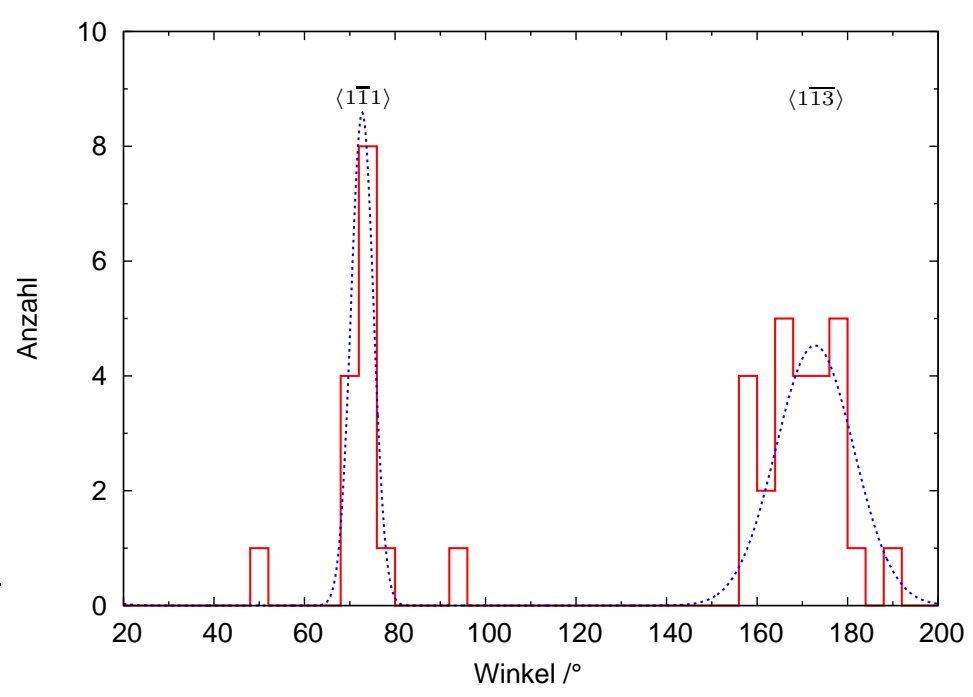

Abbildung 4.19: Winkelverteilung der Gleitstufen eines $50 \mathrm{~nm}$ dünnen Niobfilms von Abb. 4.17 (s. S. 67). Die beiden bevorzugten Richtungen lassen sich anhand des in Abschnitt 4.2.6 bestimmten Gleitsystems eindeutig den $\langle 1 \overline{1} 1\rangle$ - und $\langle 1 \overline{13}\rangle$-Richtungen zuordnen.

in die $\alpha$-Phase hinein. Die Stufen entlang der $\langle\overline{1} 13\rangle$-Richtung entstehen durch Versetzungswanderung in der $(1 \overline{2} 1)$-Ebene. Wenige Stufen sind auch in der $\langle 1 \overline{1} 1\rangle$-Richtung sichtbar. Die Stufen in dieser Richtung reichen bis zu $75 \mathrm{~nm}$ in die $\alpha$-Phase. Versetzungen, die in der (011)-Ebene wandern, hinterlassen eine Stufe entlang der $\langle 1 \overline{1} 1\rangle$ Richtung an der Oberfläche.

\subsection{Keimbildung und Wachstum}

Nachdem gezeigt wurde, dass zwei verschiedene Ausscheidungstypen existieren und deren Unterschiede dargestellt wurden, soll nun die Hydridentstehung und das Wachstum der Ausscheidungen charakterisiert werden.

\subsubsection{Anfangsstadien}

Die zeitliche Entwicklung der Hydridausscheidungen zu Beginn der Phasenumwandlung ist in Abb. 4.20 dargestellt. Der Anteil der Hydridphase ist für alle gezeigten Bilder geringer als 20\%. In dieser Abbildung sind sechs STM-Aufnahmen einer $70 \mathrm{~nm}$ dünnen Niobschicht auf einem Saphirsubstrat mit einem Miscut kleiner als $0.2^{\circ}$ enthalten. Der Messzeitpunkt ist in der Bildunterschrift für die Einzelbilder angegeben und der Zeitnullpunkt entspricht dem Beginn der Wasserstoffbeladung. Die erste Aufnahme (Teil (a)) wurde bei einem Wasserstoffdruck von $3 \cdot 10^{-7}$ mbar vor dem Beladungsbeginn aufgenommen. Auf diesem Bild existieren noch keine Hydridausscheidungen. Dann wurde der Wasserstoffdruck auf $1 \cdot 10^{-6}$ mbar erhöht. In der nachfolgenden Mes- 


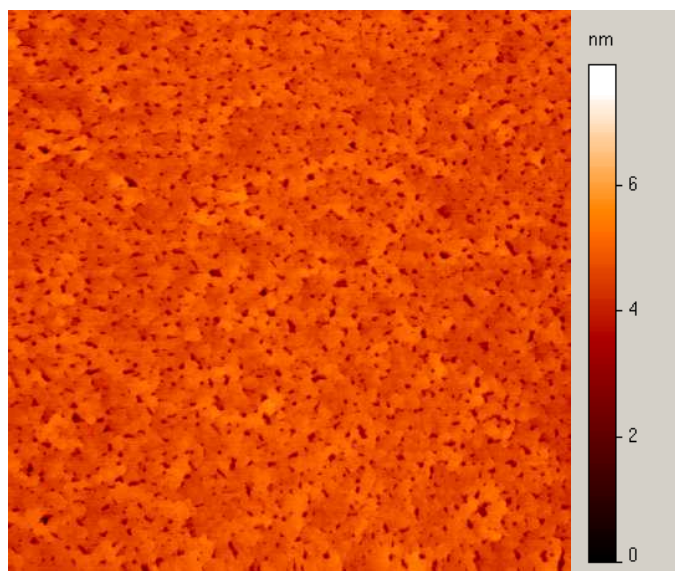

(a) $-16 \min , 3 \cdot 10^{-7}$ mbar $\mathrm{H}_{2}$

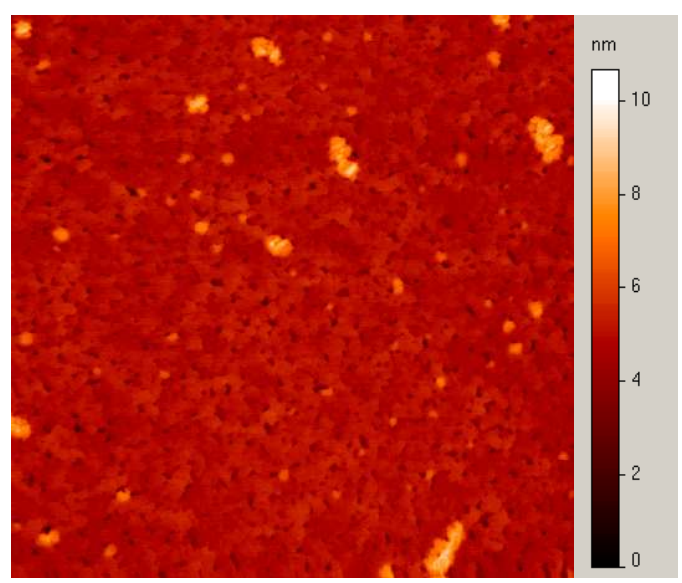

(c) $23 \mathrm{~min}, 1 \cdot 10^{-6}$ mbar $\mathrm{H}_{2}$

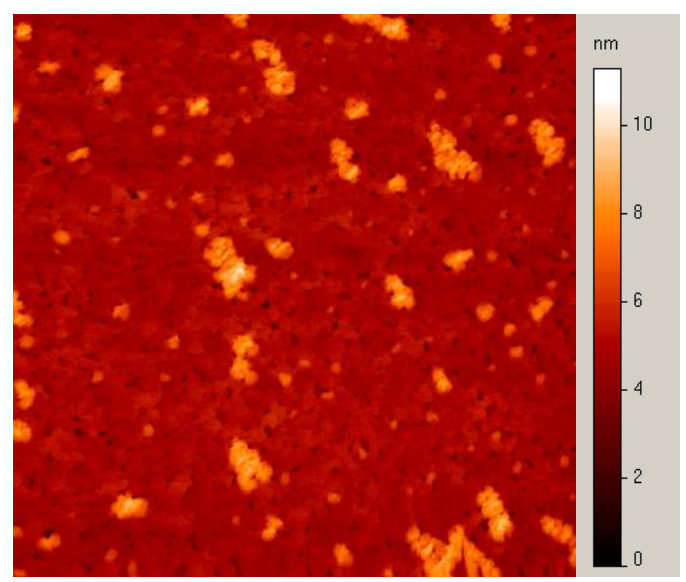

(e) $62 \mathrm{~min}, 1 \cdot 10^{-6} \mathrm{mbar}_{2}$

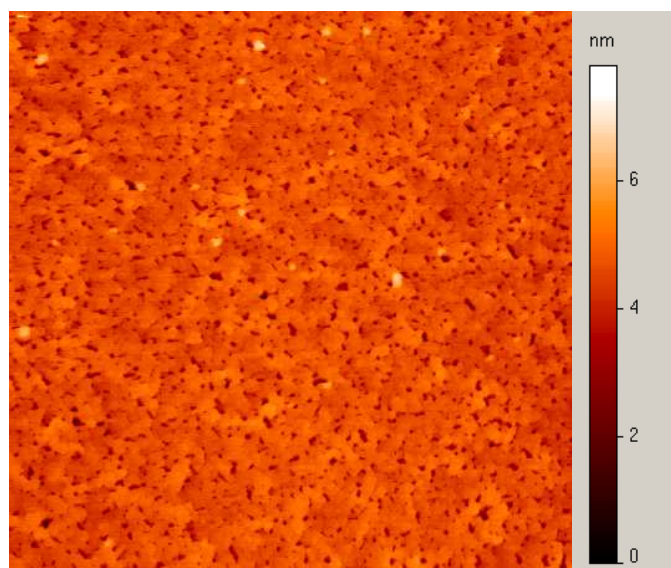

(b) $4 \mathrm{~min}, 1 \cdot 10^{-6} \mathrm{mbar} \mathrm{H}_{2}$

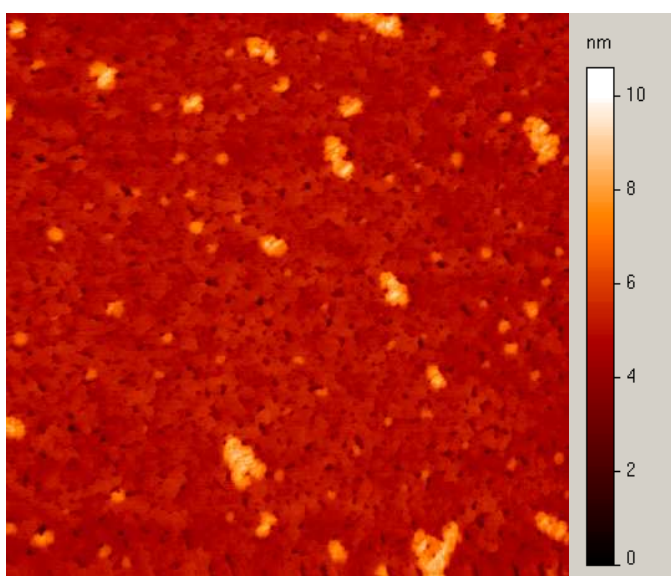

(d) $43 \mathrm{~min}, 1 \cdot 10^{-6} \mathrm{mbar} \mathrm{H}_{2}$

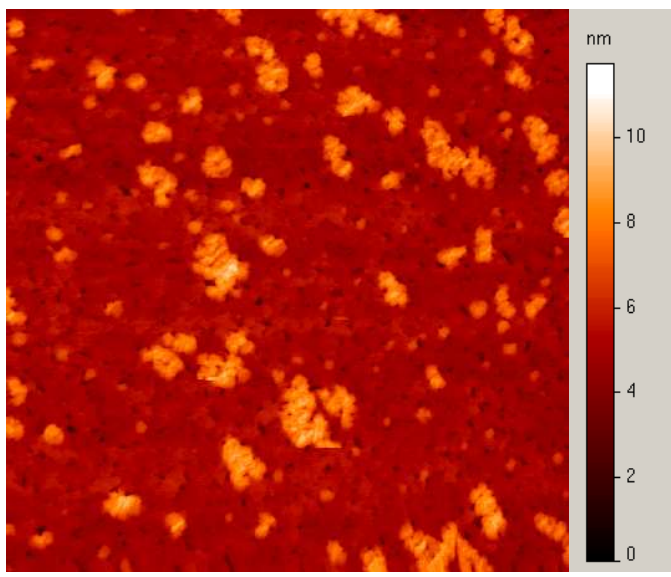

(f) $82 \mathrm{~min}, 2.5 \cdot 10^{-6} \mathrm{mbar}_{2}$

Abbildung 4.20: Zeitliche Entwicklung des Hydridwachstums bei einer $70 \mathrm{~nm}$ dünnen Niobschicht. Es sind STM-Bilder zu unterschiedlichen Zeiten der Wasserstoffbeladung dargestellt. Die Größe der Bilder beträgt $3.5 \times 3.5 \mu \mathrm{m}^{2}$. Bild (a) zeigt die Schicht vor der Wasserstoffbeladung. In Bild (b) sind die ersten Ausscheidungen vom Typ T1 zu sehen. In den darauffolgenden Bildern (c) - (f) sind Ausscheidungen vom Typ T2 erkennbar. 


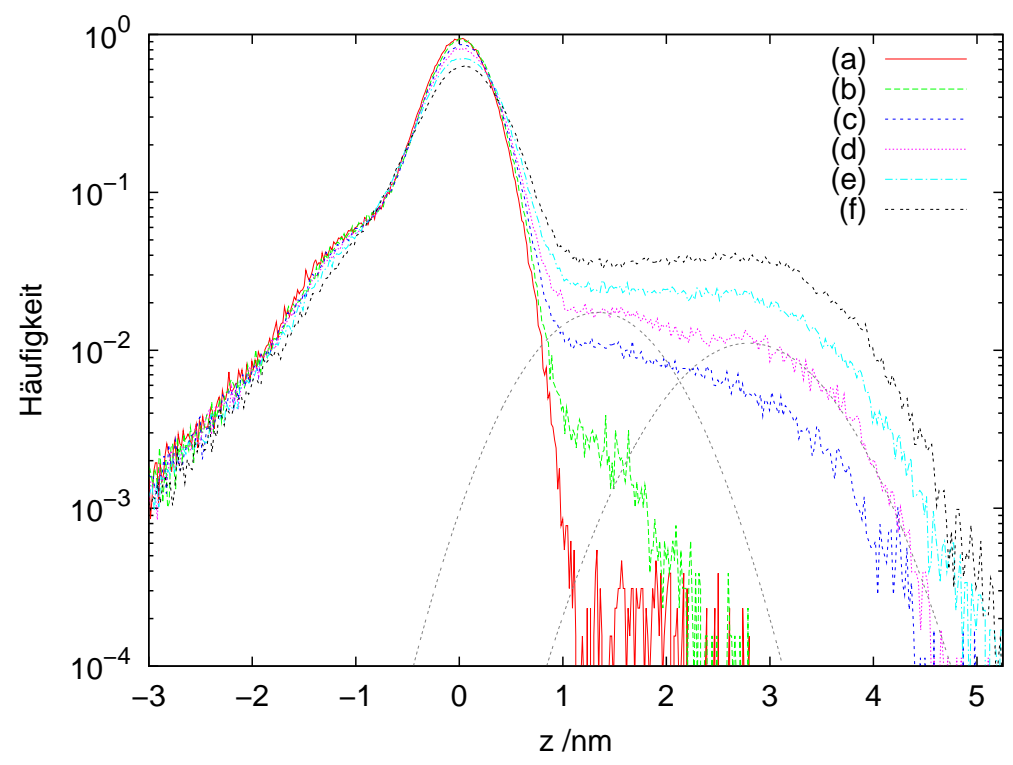

Abbildung 4.21: Höhenverteilungen der STM-Bilder einer $70 \mathrm{~nm}$ dünnen Niobschicht aus Abb. 4.20. Bei allen Graphen sind die Höhen der $\alpha$-Phase am Häufigsten vertreten. In Abb. 4.20(b) sind zusätzlich zur $\alpha$-Phase Ausscheidungen vom Typ T1 erkennbar, die im Histogramm (b) zusätzliche Höhen erzeugen. In (c) treten die Höhen auf, die den Typ T2 repräsentieren. Für den Graphen (d) sind zusätzlich noch die Anpassungen durch Gaußverteilungen für die beiden Ausscheidungstypen eingezeichnet. Der Anteil der Hydridausscheidungen nimmt vom Graphen (b) bis (f) kontinuierlich zu.

sung (b) sind die ersten Ausscheidungen vom Typ T1 sichtbar. Ausscheidungen vom Typ T2 treten in den darauffolgenden Bildern (c) - (f) auf. Der Übergang zwischen den beiden Ausscheidungstypen wird in Abschnitt 4.3.2 behandelt. Dabei wird sich zeigen, dass beide Ausscheidungstypen in z-Richtung durch die komplette Schicht reichen und der gemessene Flächenanteil dem Volumenanteil entspricht.

In den STM-Aufnahmen ist der Anstieg des Volumenanteils der Hydridausscheidungen am Gesamtvolumen $\mathrm{zu}$ erkennen. Deutlicher ist das Wachstum in der Höhenverteilung aus Abbildung 4.21. Im Histogramm des Bildes (a) gibt es nur Höhen, die von der $\alpha$-Phase stammen. Im Datensatz (b) treten die Höhen oberhalb von $1.5 \mathrm{~nm}$ auf. Diese stammen von den Ausscheidungen vom Typ T1, die im STM-Bild sichtbar sind. In den weiteren Histogrammen gibt es Höhen oberhalb von $3 \mathrm{~nm}$, die durch Ausscheidungen vom Typ T2 hervorgerufen werden. Die unterschiedliche Höhe der beiden Ausscheidungstypen ist durch das Einzeichnen von zwei Gaußverteilungen für den Graphen (d) verdeutlicht. Diese beiden Kurven sind dadurch bestimmt, dass die gemessene Höhenverteilung durch die Verteilung der $\alpha$-Phase sowie zwei Gaußkurven für die beiden Ausscheidungstypen angepasst worden ist. Der Anteil an den Höhen von mehr als $1.5 \mathrm{~nm}$ steigt kontinuierlich durch das Wachsen der Ausscheidungen an.

Anhand der Flächen unter den Histogrammkurven, die durch die unterschiedlichen Phasen hervorgerufen werden, kann der Volumenanteil dieser Phase bestimmt wer- 


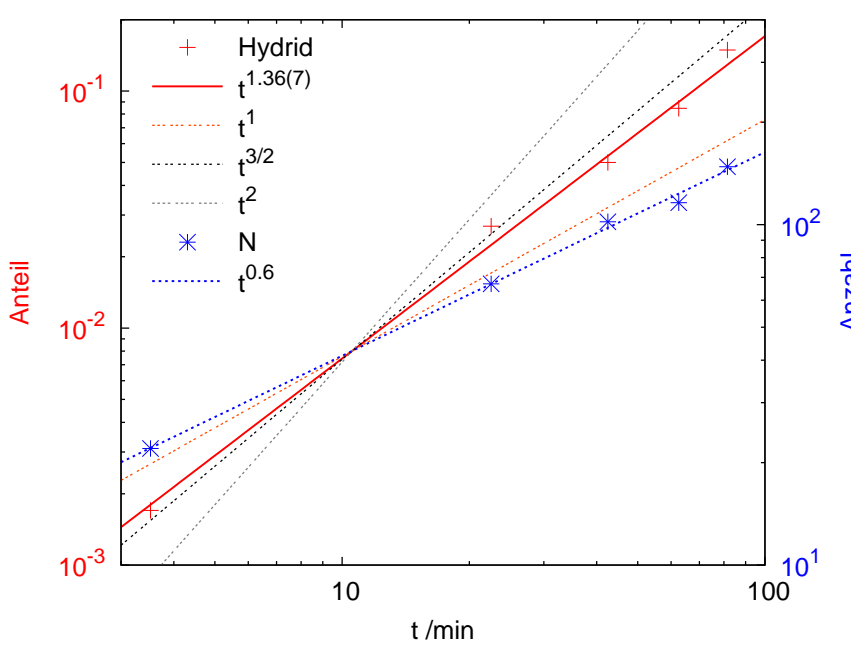

(a) Messung

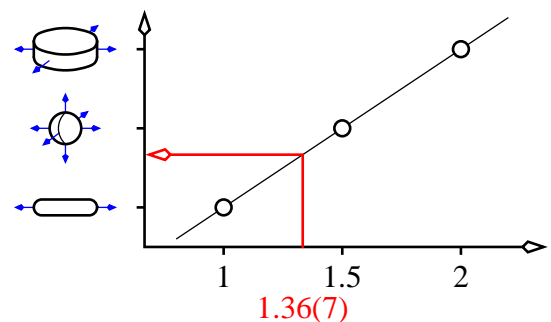

(b) Exponenten des Ausscheidungswachstums aus [Haa96]

Abbildung 4.22: Zeitliche Entwicklung des Hydrids des $70 \mathrm{~nm}$ dünnen Niobfilms aus Abb. 4.20. Der Hydridanteil ist aus den Flächen unter den Histogrammkurven in Abb. 4.21 bestimmt worden. Die Anpassung ergibt eine Abhängigkeit von $t^{1.36(7)}$ für das Hydridwachstum. Die Anzahl der Ausscheidungen steigt über den betrachteten Bereich an. Des Weiteren sind noch Abhängigkeiten für das dreidimensionale Ausscheidungswachstum eingetragen [Haa96], hierbei wachsen Zylinder mit $t^{1}$, Kugeln mit $t^{3 / 2}$ und Platten mit $t^{2}$.

den. Der ausgeschiedene Anteil $X(t)$ wird zu Beginn der Keimbildung und des Wachstums (für $X(t)<0.2$ ) durch ein Potenzgesetz $X(t) \sim t^{n}$ beschrieben [Haa96]. Das Wachstum des Hydridanteils $X(t)$, als Summe der beiden Typen T1 und T2, ist in Abb. 4.22(a) aufgetragen. Dort ist der Anteil $X(t)$ und die Anzahl $N(t)$ der Hydridausscheidungen in Abhängigkeit von der Zeit aufgetragen. Der Hydridanteil wächst mit der Zeitabhängigkeit $X(t)=(t / 367(55) \min )^{1.36(7)}$. Der hier für dünne Schichten bestimmte Exponent $n=1.36(7)$ liegt zwischen den Werten von Zylindern und Kugeln, die in massiven Proben gefunden werden (vgl. Abb. 4.22(b)) [Haa96]. Die Anzahl $N$ der Ausscheidungen ist zu $t^{0.6}$ proportional.

\subsubsection{Umwandlung der Ausscheidungstypen}

In der Abbildung 4.20 ist zu sehen, dass sich zuerst die Ausscheidungen vom Typ T1 bilden. Diese Ausscheidungen werden im Laufe der Beladung größer. Die Einstellungen der STM-Messung sind so gewählt worden, dass es möglich ist, die Entwicklung eines großen Ausschnittes beobachten zu können. Damit ist es nicht möglich, genaue Aussagen über die Entwicklung einer einzelnen Ausscheidung zu treffen. Durch den großen Ausschnitt kann aber mit statistischen Mitteln die Entwicklung der Ausscheidungen beschrieben werden, da jede einzelne Ausscheidung zu einem anderen Zeitpunkt ab- 


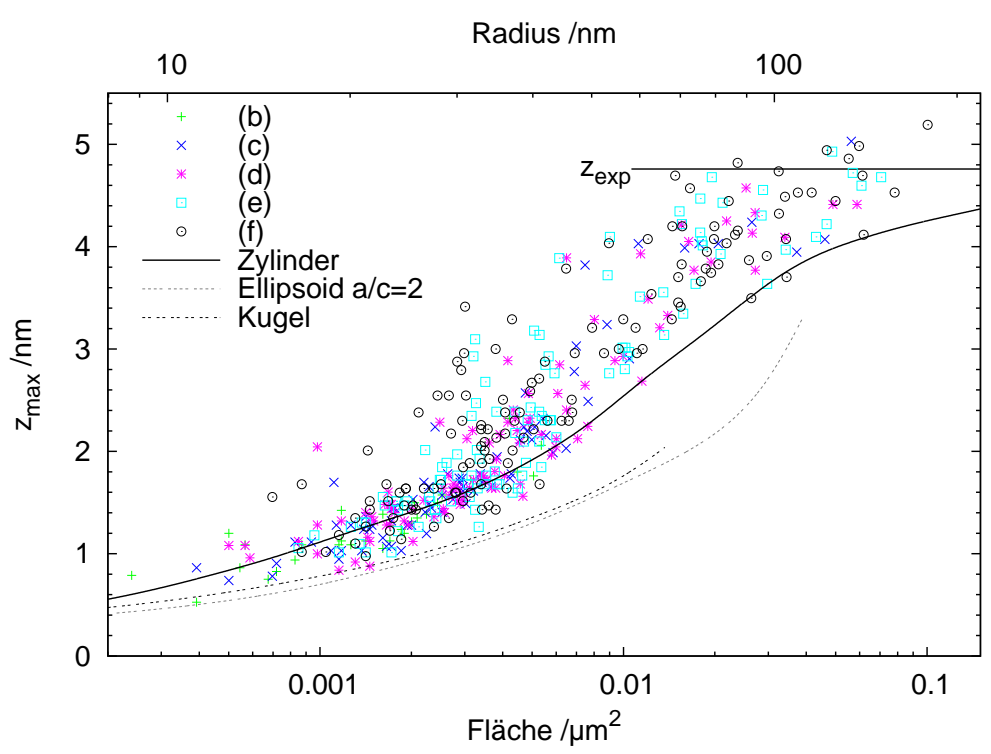

Abbildung 4.23: Maximalhöhe der Ausscheidungen aus Abb. 4.20 in Abhängigkeit von ihrer Fläche. Als zweite $x$-Achse ist noch der Radius eines Kreises mit der gleichen Fläche dargestellt. In den Graphen sind Ergebnisse einer Rechnung mit der Finite-ElementeMethode für die Ausscheidungsdehnung von Zylindern, Kugeln und Ellipsoiden eingetragen. Für Flächen $<0.004(1) \mu m^{2}$ stimmen die Messungen gut mit dem Verlauf für zylinderförmige Ausscheidungen überein. Für große Ausscheidungen nähert sich der Wert dem theoretisch erwarteten von $4.8 \mathrm{~nm}$ an.

gebildet wurde. So ist in Abb. 4.23 in Abhängigkeit der Fläche jeder Ausscheidung die maximale Höhe derselben aufgetragen.

Die kleinste beobachtete Ausscheidung hat eine Größe von ca. $2.2 \cdot 10^{-4} \mu^{2}$, dies entspricht 10 Pixeln des STM-Bildes. Kleinere Ausscheidungen können aufgrund der gewählten Bildparameter nicht detektiert werden. Der geringste Höhenunterschied beträgt $0.5 \mathrm{~nm}$. Von dieser Aufösungsgrenze steigt die Höhe langsam mit der Ausscheidungsfläche an. Dieser Höhenanstieg wird ab einer Fläche von $0.004(1 / 2) \mu \mathrm{m}^{2}$ stärker. Die maximale Höhenänderung wird ab einer Fläche von $0.05(1 / 2) \mu \mathrm{m}^{2}$ erreicht. Ab dieser Ausscheidungsgröße wird der nach der linearen Elastizitätstheorie für $\sigma_{z}=0$ erwartete Wert von $z=4.8 \mathrm{~nm}$ gemessen (vgl. Gl. (2.10), S. 8). In den Graphen sind neben den experimentell bestimmten Daten zusätzlich noch die Ergebnisse der Berechnung anhand der Finite-Element-Methode mit drei verschiedenen Ausscheidungsgeometrien (Zylinder, Ellipsoid und Kugel) eingezeichnet. Diese Berechnung ist in Abschnitt 3.3 beschrieben und die Daten sind in der Abb. 3.21 auf Seite 40 dargestellt. Bis zu einer Fläche von 0.004(1/2) $\mathrm{mm}^{2}$ stimmt der Verlauf der Messdaten mit den Werten für kohärente zylinderförmige Ausscheidungen gut überein. Dieser Fläche entspricht ein Radius von $r=36(3) \mathrm{nm}$. Bei größeren Ausscheidungen liegen die berechneten Werte unterhalb der experimentell bestimmten Daten. Mit kugelförmigen und ellipsoiden Ausscheidungen (gestrichelte Linien in Abb. 4.23) werden die gemessenen Höhenänderungen für keine Ausscheidungsgröße erreicht. Bei den Ausscheidungen 


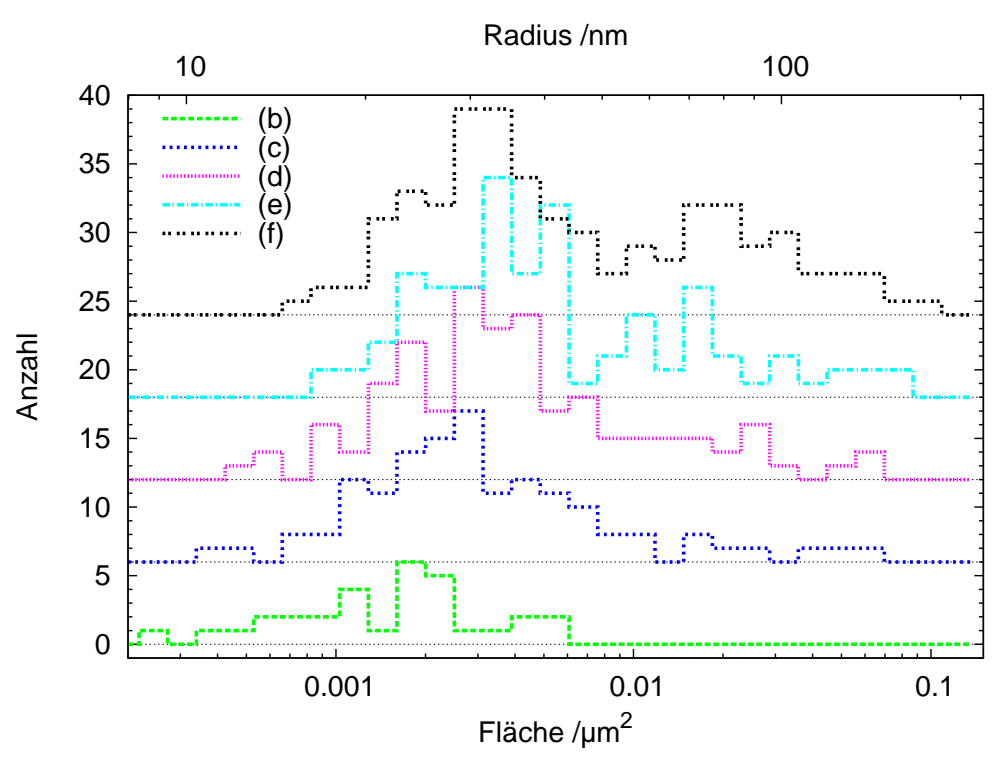

Abbildung 4.24: Häufigkeitsverteilung der Größe der Ausscheidungen der STM-Bilder aus Abb. 4.20. Der Aufnahmezeitpunkt der einzelnen Histogramme sind (b): 4 min, (c): 23 min, (d): 43 min, (e): 62 min und (f): 81 min. Im Frühstadium (b) sind nur Ausscheidungen mit einer Fläche kleiner als $0.0054 \mu \mathrm{m}^{2}$ vorhanden. Bei späteren Aufnahmen steigt die Anzahl der beobachteten Ausscheidungen und die einzelnen Ausscheidungen werden größer.

vom Typ T1 handelt es sich somit um kohärente Zylinder, die in $z$-Richtung bis zum Substrat reichen.

Das laterale Wachstum der Ausscheidungen wird in Abb. 4.24 gezeigt. Dort sind für die einzelnen STM-Bilder aus Abb. 4.20 Histogramme der Ausscheidungsgröße erstellt. Im Histogramm von Teilbild (b) wird deutlich, dass eine Ausscheidungsfläche von $0.002 \mu \mathrm{m}^{2}$ am häufigsten auftritt. Ausscheidungen größer als $0.0054 \mu \mathrm{m}^{2}$ sind in diesem Bild nicht vorhanden. In der anschließend aufgenommenen Messung (c) liegt die häufigste Ausscheidungsfläche bei $0.003 \mu \mathrm{m}^{2}$. Es treten Ausscheidungen bis zu einer Größe von $0.07 \mu^{2}$ auf. Das Maximum der Histogramme steigt in den folgenden Graphen bis zu einer Fläche von $0.004 \mu \mathrm{m}^{2}$ im Histogramm von Bild (f) an. Des Weiteren steigt auch die Fläche der größten Ausscheidung in den Graphen mit der Zeit stetig bis zu einem Wert von $0.1 \mu \mathrm{m}^{2}$ in Bild (f) an. In den nach Bild (f) aufgenommenen STM-Bildern steigt die Fläche der Ausscheidungen so lange weiter an, bis die einzelnen Ausscheidungen ineinander gewachsen sind. Abbildung 4.24 verdeutlicht, dass die Größe der Ausscheidungen mit der Zeit ansteigt, wobei auf allen Bildern bei einer Fläche, die kleiner als $0.004 \mu \mathrm{m}^{2}$ ist, ein Häufigkeitsmaximum auftritt. Diese Größe entspricht der Fläche, bei der die Höhe der Ausscheidungen nicht mehr mit den Ergebnissen der Finite-Elemente-Methode übereinstimmt (vgl. Abb. 4.23). Sie liegt in dem Größenbereich, in dem die Ausscheidungen vom Typ T2 auftreten und die Ausscheidungen vom Typ T1 nicht mehr sichtbar sind. 


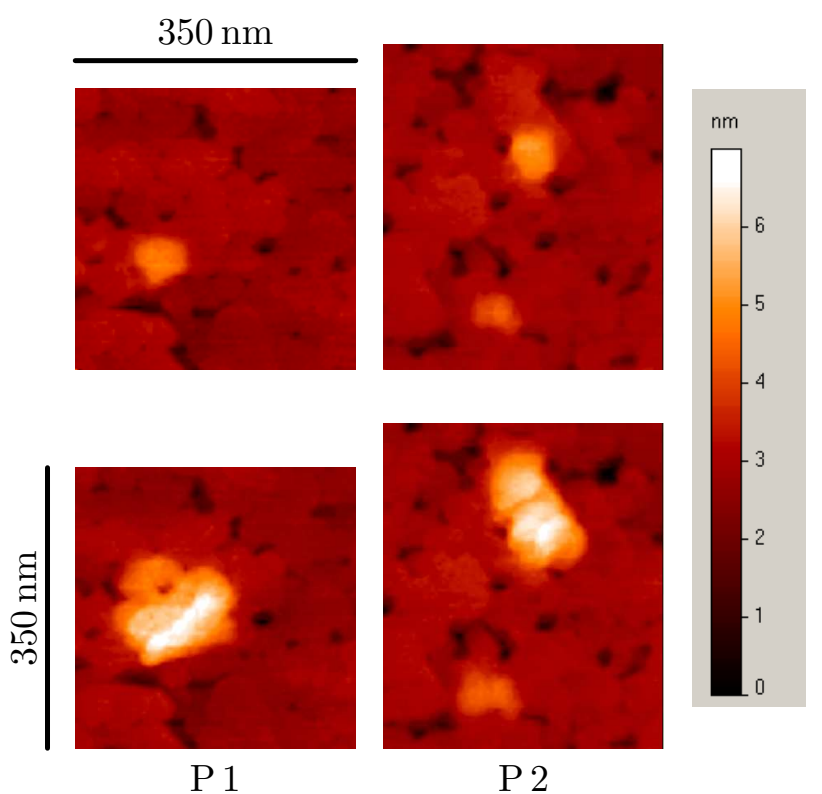

Abbildung 4.25: Umwandlung der Ausscheidungen vom Typ T1 zum Typ T2. Abgebildet sind Ausschnitte der STM-Topographiebilder aus Abb. 4.20(c) und 4.20(d) an zwei unterschiedlichen Positionen (P 1 und $\mathrm{P} 2$ ). In den beiden oberen Bildern sind Typ T1-Auscheidungen zu sehen. Auf den späteren Ausschnitten (untere Bilder) sind an denselben Positionen Typ T2-Ausscheidungen.

Bei dieser Größe findet die Umwandlung der Ausscheidungen vom Typ T1 zum Typ T2 statt. An Positionen, an denen anfangs Ausscheidungen vom Typ T1 beobachtet wurden, haben sich bei späteren Messungen Typ T2 Ausscheidungen gebildet. Dies ist in Abb. 4.25 zu erkennen, in der Ausschnittsvergrößerungen von Abb. 4.20(c) bzw. Abb. 4.20(d) dargestellt sind. In den oberen Abbildungen sind Ausscheidungen vom Typ T1 vorhanden. Diese haben eine geringe Erhöhung gegenüber der $\alpha$ Phase und weisen keine Gleitstufen auf. Zu einem späteren Zeitpunkt (untere Abbildungen) haben sich die Ausscheidungen lateral vergrößert und ein Höhenwachstum hat stattgefunden. Es haben sich Gleitstufen gebildet. Aus den kohärenten Typ T1Ausscheidungen sind inkohärente Typ T2-Ausscheidungen entstanden.

\subsubsection{Zeitliche Entwicklung der Hydridausscheidungen bis $3 \mathrm{~h}$}

Bei der Beladung, die in Abschnitt 4.3.1 beschrieben worden ist, wurde der Anfangsbereich der Hydridbildung beobachtet. Das Wachstum ließ sich mit einem Potenzgesetz beschreiben. Wenn sich die Einzugsbereiche der unterschiedlichen Ausscheidungen überlagern, ist ein Potenzgesetz nicht mehr gültig. Die zeitliche Zunahme des ausgeschiedenen Anteils $X(t)$ lässt sich dann durch eine Johnson-Mehl-Avrami Kinetik der Form

$$
X(t)=j\left(1-\exp \left\{-\left(\frac{t}{\tau}\right)^{n}\right\}\right)
$$

beschreiben. Bei dieser Abhängigkeit ist $j$ der Anteil, der sich nach sehr langer Zeit als Gleichgewichtswert einstellt. Die Zeitabhängigkeit wird durch die beiden Parameter $\tau$ und $n$ beschrieben, dabei geht der Wachstumsmodus in den Wert $n$ ein. Bei 


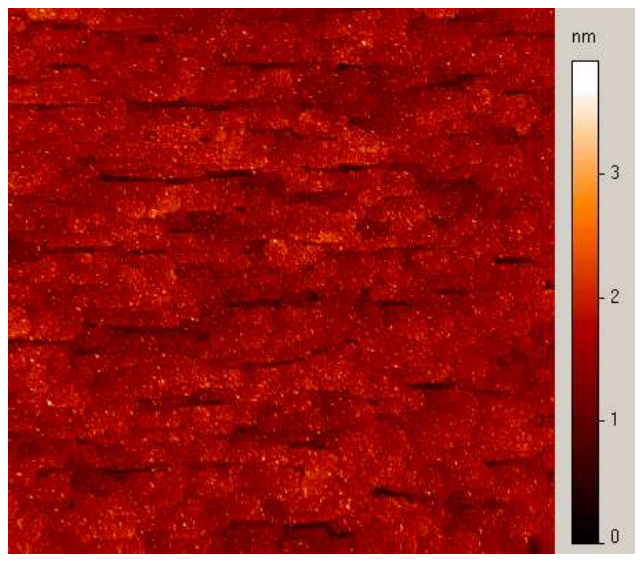

(a) $-18 \min , 7 \cdot 10^{-7}$ mbar $\mathrm{H}_{2}$

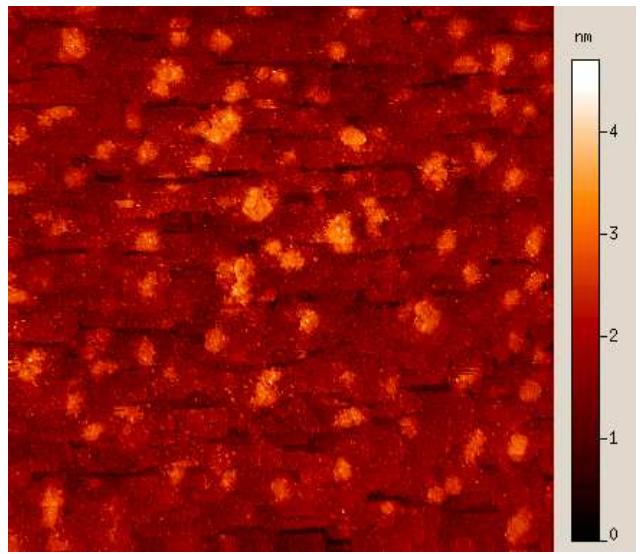

(c) $39 \mathrm{~min}, 1 \cdot 10^{-6} \mathrm{mbar}_{2}$

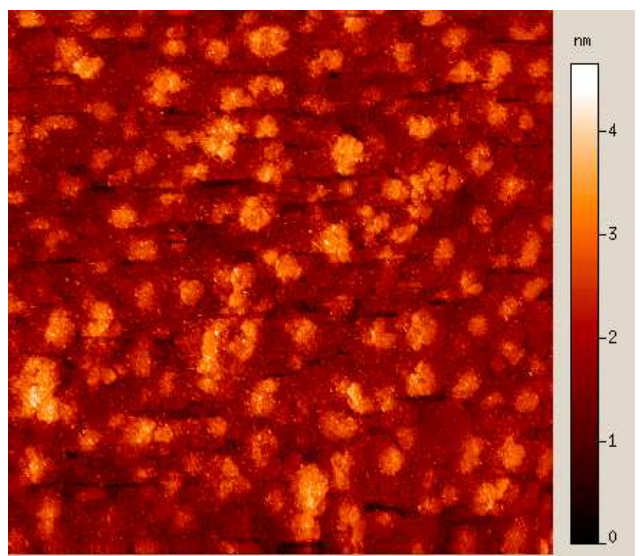

(e) $114 \mathrm{~min}, 4 \cdot 10^{-6} \mathrm{mbar} \mathrm{H}_{2}$

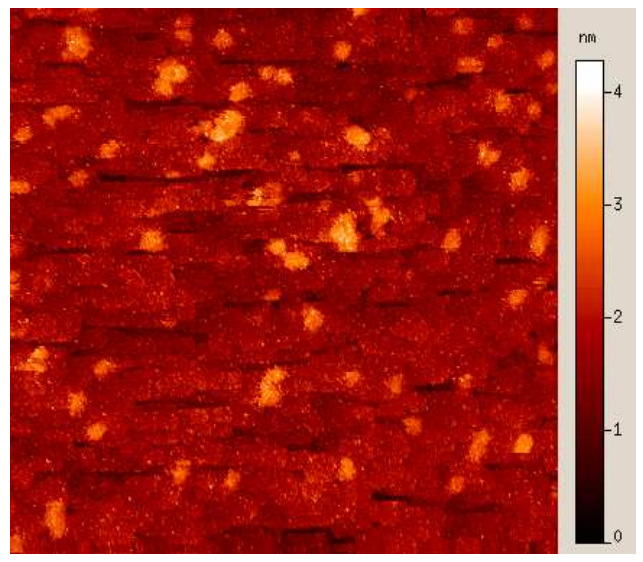

(b) 20 min, $1 \cdot 10^{-6}$ mbar $\mathrm{H}_{2}$

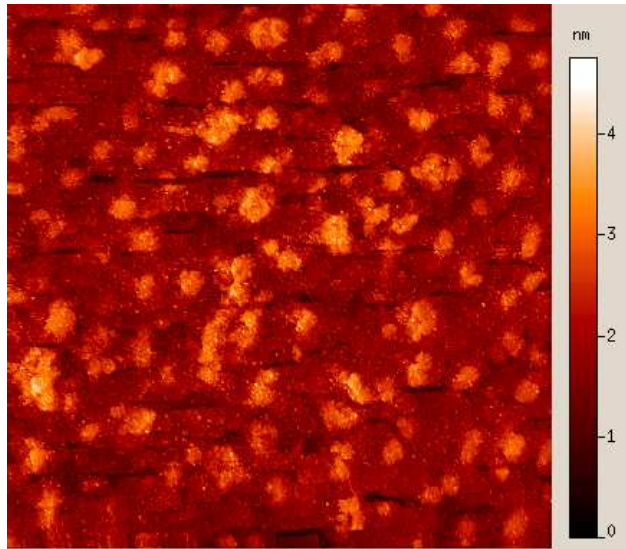

(d) $76 \mathrm{~min}, 1 \cdot 10^{-6} \mathrm{mbar} \mathrm{H}_{2}$

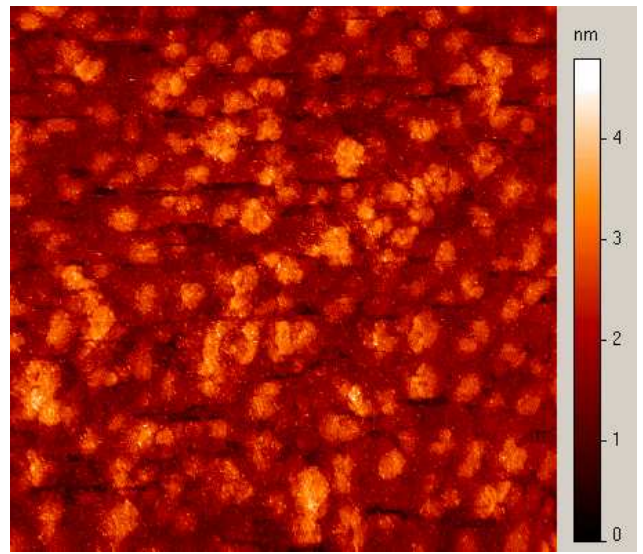

(f) $133 \mathrm{~min}, 4 \cdot 10^{-6} \mathrm{mbar}_{2}$

Abbildung 4.26: STM-Bilder eines $30 \mathrm{~nm}$ dünnen Niobfilms bei unterschiedlichen Wasserstoffdrücken. Die einzelnen Bilder zeigen einen Ausschnitt von $1 \times 1 \mu \mathrm{m}^{2}$. In (a) ist die Schicht bei einem $\mathrm{H}_{2}$-Druck von $7 \cdot 10^{-7}$ mbar vor der Hydridbildung dargestellt. Bei einem Druck von $1 \cdot 10^{-6}$ mbar (b)-(d) wird Hydrid langsam gebildet, und der Anteil wächst mit der Zeit. Bei späteren Aufnahmen (e) und (f) steigt der Hydridanteil nur wenig an. Die genaue zeitliche Veränderung ist in Abb. 4.27 aufgetragen. 


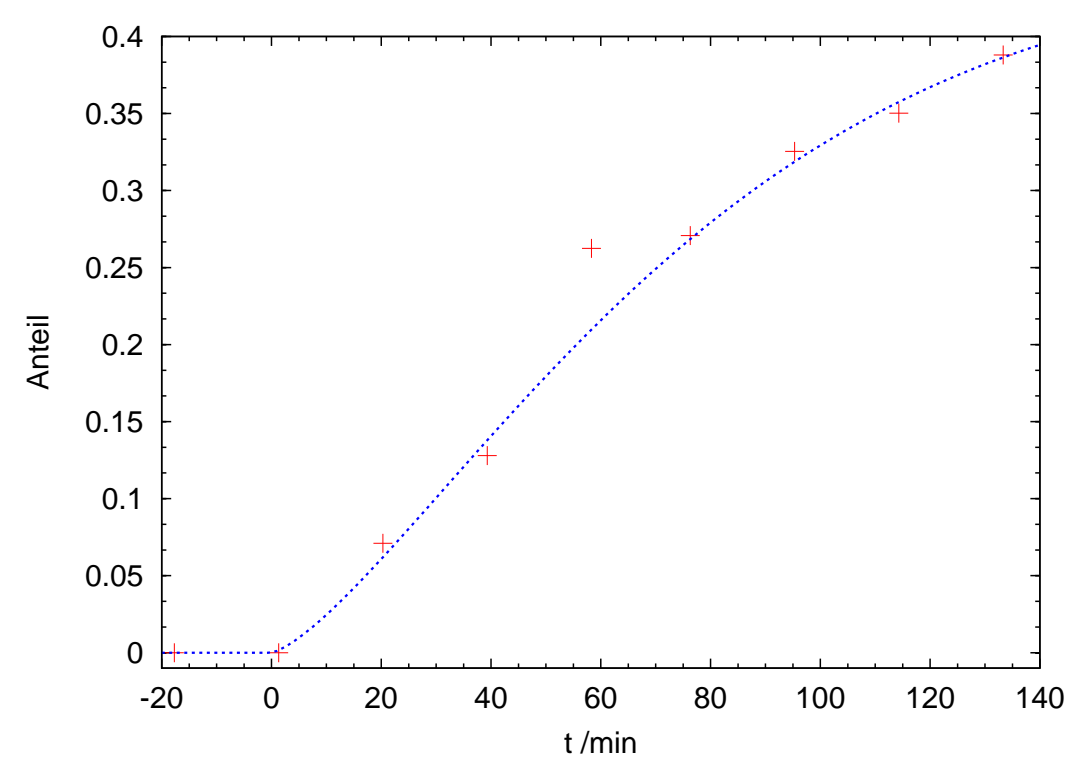

Abbildung 4.27: Hydridwachstum eines $30 \mathrm{~nm}$ dünnen Niobfilms. Die Topographiebilder dieser Schicht sind in Abb. $4.26 \mathrm{zu}$ finden. Aufgetragen ist die zeitliche Änderung des Hydridanteils. Die Messwerte lassen sich mit einer Johnson-Mehl-Avrami Kinetik (Gleichung (4.3)) mit $j=0.45(4), \tau=82(9)$ min und $n=1.37(10)$ anpassen.

J. W. Christian [Chr65, Seite 489] ist eine Zusammenstellung von verschiedenen Werten $n$ und zugehörigen Wachstumsmodi zu finden.

In Abb. 4.26 sind STM-Aufnahmen einer $30 \mathrm{~nm}$ dünnen Niobschicht auf einem Substrat dargestellt, dessen Miscut geringer als $0.2^{\circ}$ ist. Bei dem Bild (a) war ein Wasserstoffdruck von $7 \cdot 10^{-7}$ mbar eingestellt und es sind noch keine Hydridausscheidungen zu beobachten. Dann wurde der Druck auf $1 \cdot 10^{-6}$ mbar $\mathrm{H}_{2}$ erhöht (bei $t=0 \mathrm{~min}$ ). In den anschließenden Bildern (b)-(f) sind Ausscheidungen zu beobachten. Die von Ausscheidungen bedeckte Fläche steigt in den aufeinanderfolgenden STM-Bildern an. Dieser ansteigende Anteil des Hydrids ist in Abb. 4.27 aufgetragen. Es wird deutlich, dass mit der Druckerhöhung bei $t=0$ min der Anteil des Hydrids mit dem zeitlichen Verlauf einer Johnson-Mehl-Avrami Kinetik ansteigt (vgl. Gl. (4.3)). Bei dieser Anpassung werden folgende Werte bestimmt:

$$
\begin{aligned}
j_{30 \mathrm{~nm}} & =45(4) \% \\
\tau_{30 \mathrm{~nm}} & =82(9) \min \\
n_{30 \mathrm{~nm}} & =1.37(10)
\end{aligned}
$$

Der Gleichgewichtswert des Anteils der Hydridphase ist geringer als eins. Dies spricht für ein thermodynamisches Gleichgewicht zwischen beiden Phasen in dünnen Schichten. Die Sättigung des Keimwachstums bzw. das Erreichen des Gleichgewichts ist in Abb. 4.27 noch nicht vollständig sichtbar. Im folgenden Abschnitt wird eine Messung beschrieben, bei der die Sättigung deutlich zu erkennen ist. Der Exponent des 
Wachstums entspricht dem Exponenten, der für eine $70 \mathrm{~nm}$ dünne Niobschicht im Anfangsstadium entdeckt wurde (vgl. Abb. 4.22(a), S. 72).

\subsubsection{Zeitliche Entwicklung der Ausscheidungen bis $170 \mathrm{~h}$}

In Abschnitt 2.2.3 (s. S. 8) wurde das thermodynamische Gleichgewicht von $\alpha$-Phase und Hydrid in dünnen Schichten behandelt. Dort wurde beschrieben, dass für elektrochemisch beladene Filme beide Phasen im Gleichgewicht nebeneinander existieren können. Hier soll überprüft werden, ob die Koexistenz der beiden Phasen durch die Kinetik erklärt wird, oder ob bei einer langsamen Gasbeladung ein Plateau in einer Isotherme auftritt. Dazu ist eine Messung durchgeführt worden, bei der der Druck über einen sehr langen Zeitraum konstant gehalten wurde. Der Anteil von $\alpha$-Phase und Hydrid ist kontinuierlich bestimmt worden. Es folgte eine Messung, bei der ein Druck von $9 \cdot 10^{-7}$ mbar eingestellt wurde, so dass sich Hydridausscheidungen mit langsamer Geschwindigkeit bilden. Um das Gleichgewicht zu erreichen, ist der Druck über einen Zeitraum von einer Woche konstant gehalten worden. Während dieser Beladungszeit sind kontinuierlich STM-Bilder erstellt worden.

Für diese Messung wurde eine $80 \mathrm{~nm}$ dünne Niobschicht verwendet, die auf einem Substrat mit einem Miscut geringer als $0.1^{\circ}$ deponiert worden ist. Eine Auswahl der aufgenommenen Bilder ist in Abb. 4.28 dargestellt. Das STM-Bild (a) wurde bei einem Druck von $2 \cdot 10^{-9}$ mbar vor Beginn der Wasserstoffbeladung gemessen. Auf diesem Bild ist die Oberflächenmorphologie der Probe vor dem Wasserstoffeinfluss zu erkennen. Auf diesem STM-Bild ist nur die $\alpha$-Phase vorhanden. Bei der Zeit $t=0$ ist ein Wasserstoffdruck von $9 \cdot 10^{-7}$ mbar eingestellt worden und im Bild (b) sind Hydridausscheidungen vom Typ T1 zu sehen. Ausscheidungen vom Typ T2 treten in dieser STM-Messung noch nicht auf. In Abb. (c) erscheinen wenige kleinere Ausscheidungen vom Typ T2. Die Anzahl der Typ T1-Ausscheidungen ist im Vergleich zu Bild (b) gestiegen. In der späteren Aufnahme (d) verändert sich die Anzahl der Typ T1-Ausscheidungen nicht mehr, aber die Anzahl der Typ T2-Ausscheidungen steigt, und die einzelnen Ausscheidungen werden deutlich größer. Im Bild (e) sind noch beide Ausscheidungstypen zu erkennen. Die Ausscheidungen bedecken ungefähr das halbe Bild. Dieses Hydridwachstum ist in Abb. 4.29 aufgetragen. In diesem Diagramm ist für jedes aufgenommene STM-Bild der Anteil von beiden Ausscheidungstypen, sowie deren Summe als Funktion der Beladungszeit aufgetragen. Es ist zu erkennen, dass zu Beginn nur Ausscheidungen vom Typ T1 vorhanden sind, deren Anteil langsam wächst. Der Anteil diesen Typs steigt bis zu 7.5\% an. Nach ungefähr 2.5 Tagen verändert sich dieser Bruchteil nicht mehr. Die Typ T2-Ausscheidungen bilden sich ca. $25 \mathrm{~h}$ nach Beladungsbeginn. Der Anteil dieser Ausscheidungen steigt mit der Zeit an. Beim ersten Auftreten des Typs T2 fällt kurzzeitig der Anteil des Typs T1 ab, was darauf zurückzuführen ist, dass sich der Typ T2 aus den Ausscheidungen vom Typ T1 bildet (vgl. Abb. 4.25, S. 75). Es ist zu erkennen, dass der Anteil der Summe beider Typen mit dem zeitlichen Verlauf einer Johnson-Mehl-Avrami Kinetik (vgl. Gl. (4.3), 


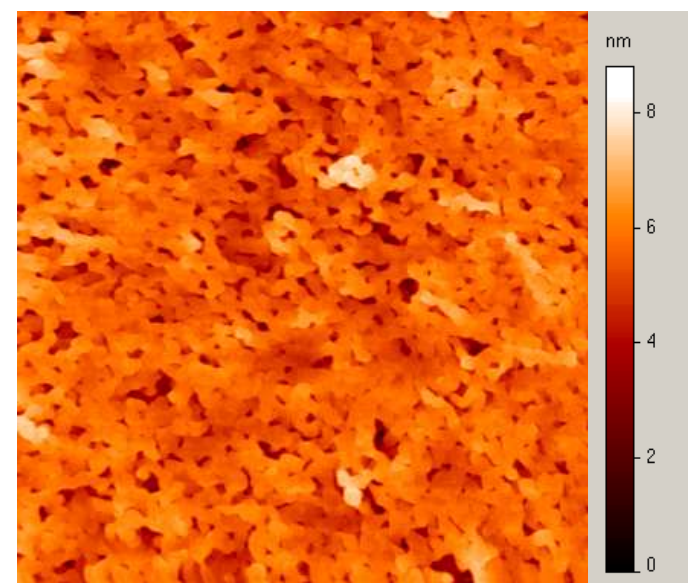

(a) $-0.5 \mathrm{~h}, 2 \cdot 10^{-9} \mathrm{mbar}$

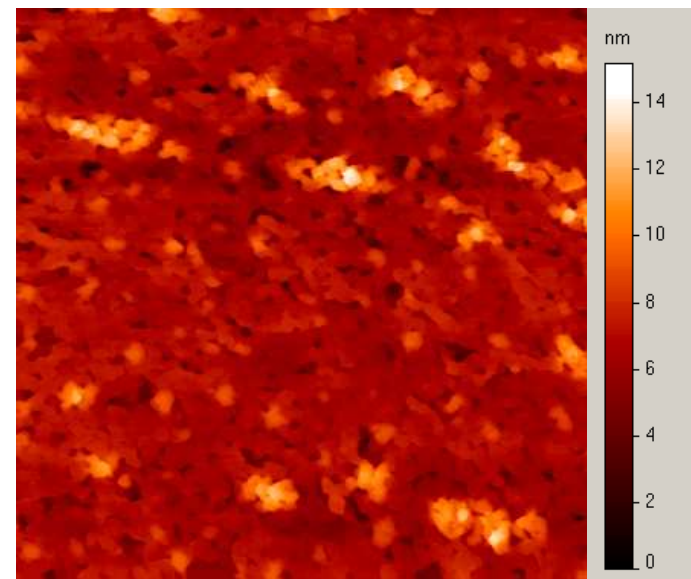

(c) $41.6 \mathrm{~h}, 9 \cdot 10^{-7} \mathrm{mbar}$

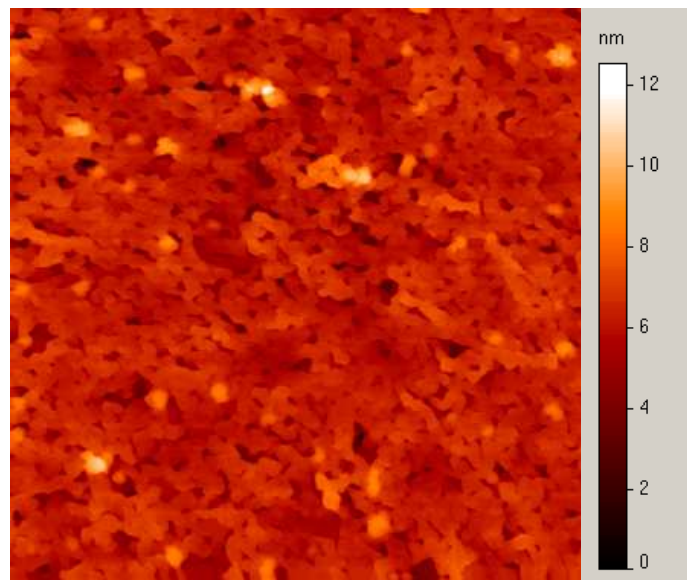

(b) $24.2 \mathrm{~h}, 9 \cdot 10^{-7} \mathrm{mbar}$

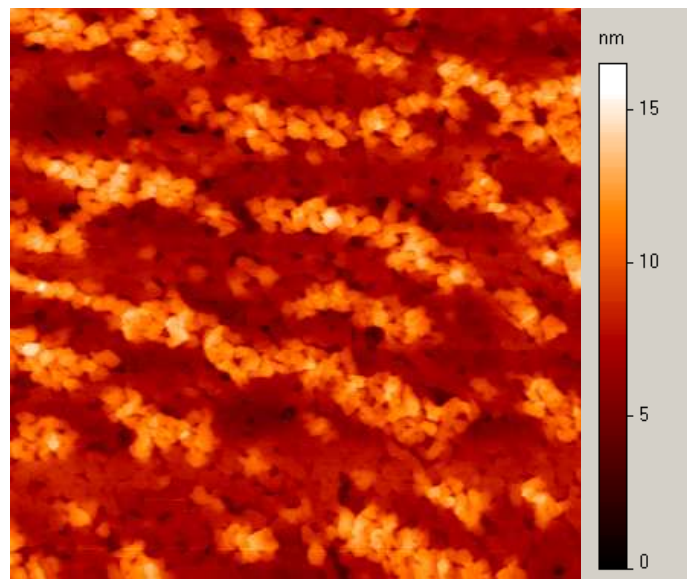

(d) $95.8 \mathrm{~h}, 9 \cdot 10^{-7} \mathrm{mbar}$

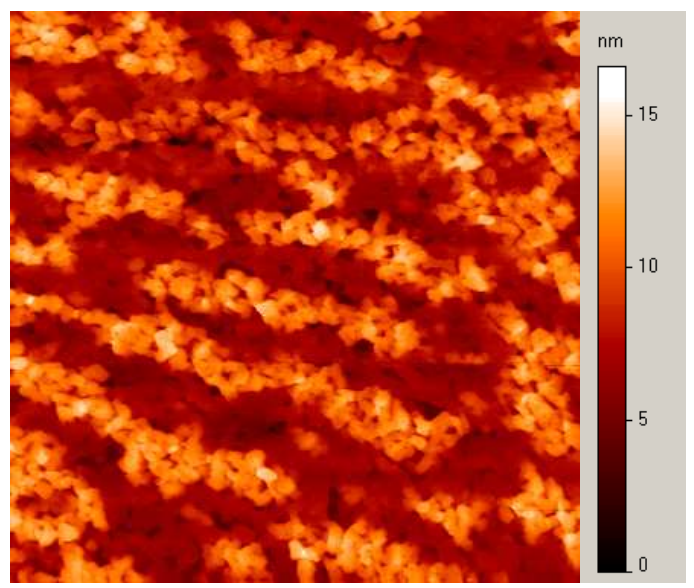

(e) $134 \mathrm{~h}, 9 \cdot 10^{-7} \mathrm{mbar}$

Abbildung 4.28: STM-Bilder der zeitlichen Entwicklung der Hydridbildung eines $80 \mathrm{~nm}$ dünnen Niobfilms. In den Bildern ist ein Ausschnitt mit einer Größe von $2.5 \times 2.5 \mu \mathrm{m}^{2}$ dargestellt. In Bild (a) ist die Schicht bei einem Druck von $2 \cdot 10^{-9}$ mbar vor der Wasserstoffbeladung dargestellt. Zur Zeit $t=0$ wurde der Druck auf $9 \cdot 10^{-7}$ mbar erhöht und in den Bildern (b)-(e) ist das Wachstum der Hydridausscheidungen zu erkennen. 


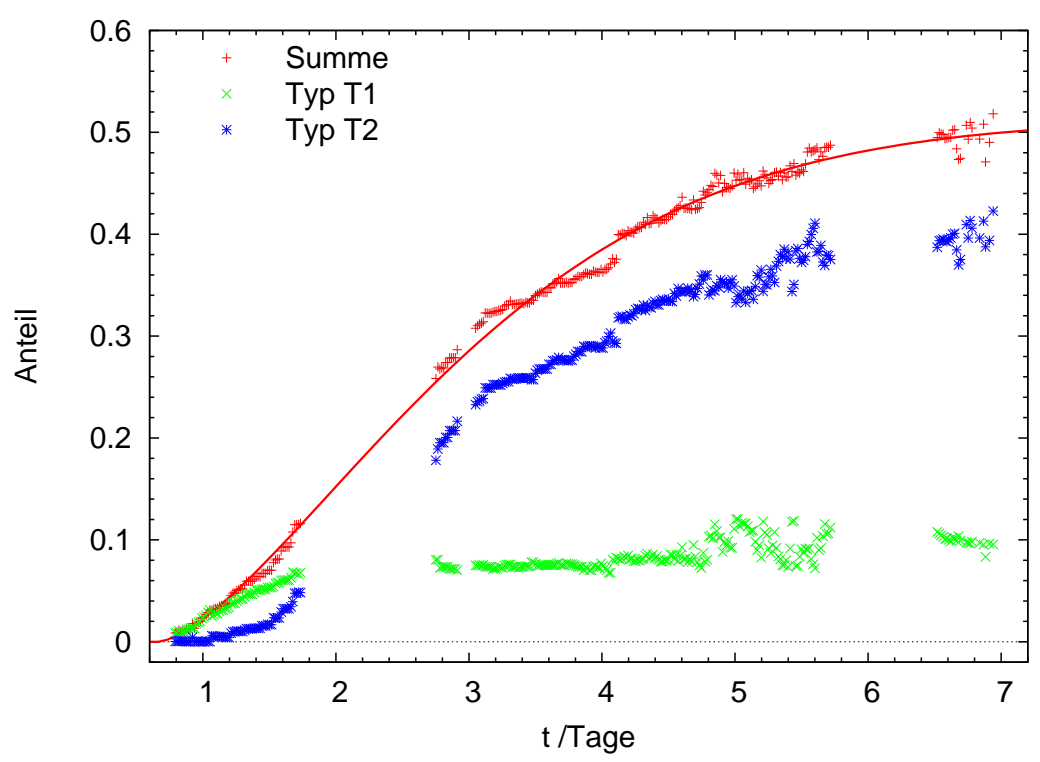

Abbildung 4.29: Ausscheidungswachstum einer $80 \mathrm{~nm}$ dünnen Niobschicht bei einem Wasserstoffdruck von $9 \cdot 10^{-7}$ mbar. Der Verlauf des Anteils der Ausscheidungen vom Typ T1, sowie die Summe beider Typen lässt sich mit einer Johnson-Mehl-Avrami Kinetik anpassen. Der Volumenbruchteil der Ausscheidungen nähert sich asymptotisch dem Wert von $51.4(3) \%$ an.

S. 75) wächst. Die Parameter für den Verlauf der Summe beider Ausscheidungstypen sind:

$$
\begin{aligned}
j_{\text {Summe }} & =51.4(3) \% \\
\tau_{\text {Summe }} & =64.4(5) \mathrm{h} \\
n_{\text {Summe }} & =1.50(2)
\end{aligned}
$$

Im zeitlichen Verlauf des umgewandelten Anteils der Schicht wird deutlich, dass die Veränderungen nach einer Beladungszeit von 7 Tagen zwischen den einzelnen Bildern sehr gering sind. Es stellt sich somit beim Druck von $9 \cdot 10^{-7}$ mbar ein Gleichgewicht der Anteile von $\alpha$-Phase und Hydridausscheidungen ein. Der Gleichgewichtswert liegt nach der Johnson-Mehl-Avrami Anpassung bei 51.4(3)\%.

\subsection{Laterale Schichtausdehnung}

Hier soll auf einen Niobfilm eingegangen werden, der eine sehr offene Struktur hat. Der Film wurde auf einem Saphirsubstrat mit einem Miscut kleiner als $0.1^{\circ}$ hergestellt. Bei einer Schichtdicke von $20 \mathrm{~nm}$ beträgt der Höhenunterschied der Oberfläche ca. $11 \mathrm{~nm}$ auf einem $2.5 \times 2.5 \mu \mathrm{m}^{2}$ großen Ausschnitt (vgl. Abb. 3.10(a), S. 28). In der Detailaufnahme in Abb. 3.10(b) ist zu sehen, dass sich kleine, sehr glatte Terrassen gebildet haben. 


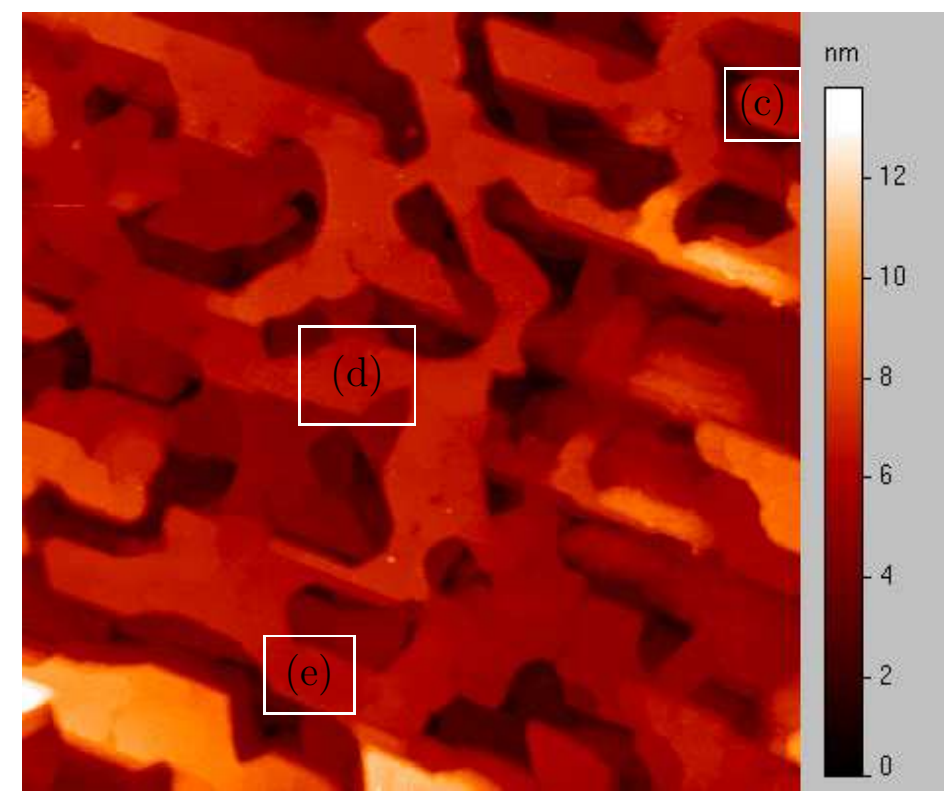

(a) vor der Wasserstoffbeladung

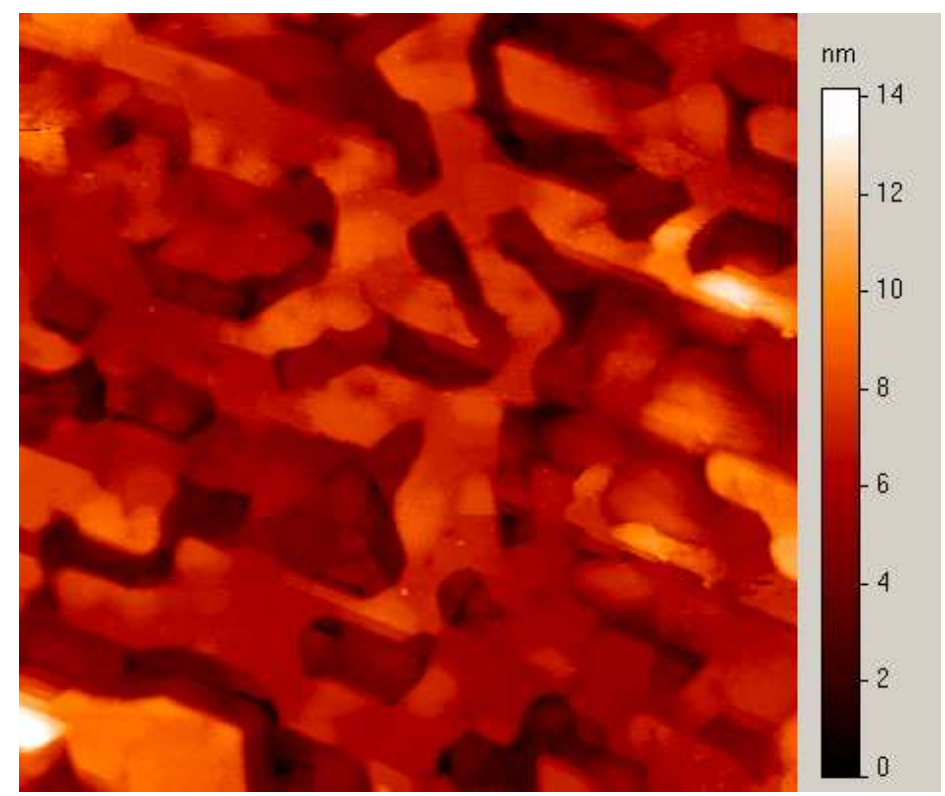

(b) bei $4.3 \cdot 10^{-7}$ mbar Wasserstoff
Differenzbilder einzelner

Ausschnitte der Aufnahmen (a) und (b)

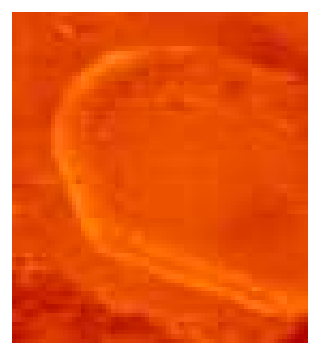

(c)

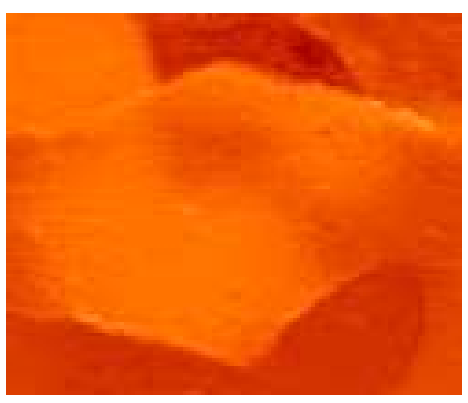

(d)

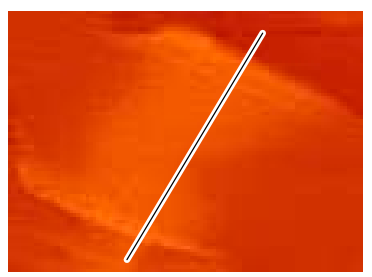

(e)

Abbildung 4.30: Veränderungen einer $20 \mathrm{~nm}$ dünnen Nb-Schicht während der Wasserstoffbeladung. In (a) ist der Film vor der Beladung abgebildet. Bei der Zugabe von Wasserstoff (b) bildet sich an einigen Stellen ein Hydrid. Da der Höhenunterschied nicht sehr groß ist, sind in (c)-(e) Differenzbilder von drei Ausschnitten dargestellt. Es ist zu sehen, dass die Terrassenränder bei diesen Differenzbildern erhöht sind.

Die Veränderungen, die bei Beladung mit einem Wasserstoffpartialdruck von $p=4.3 \cdot 10^{-7}$ mbar auftreten, sind in Abb. 4.30 erkennbar. Nach der Zugabe 


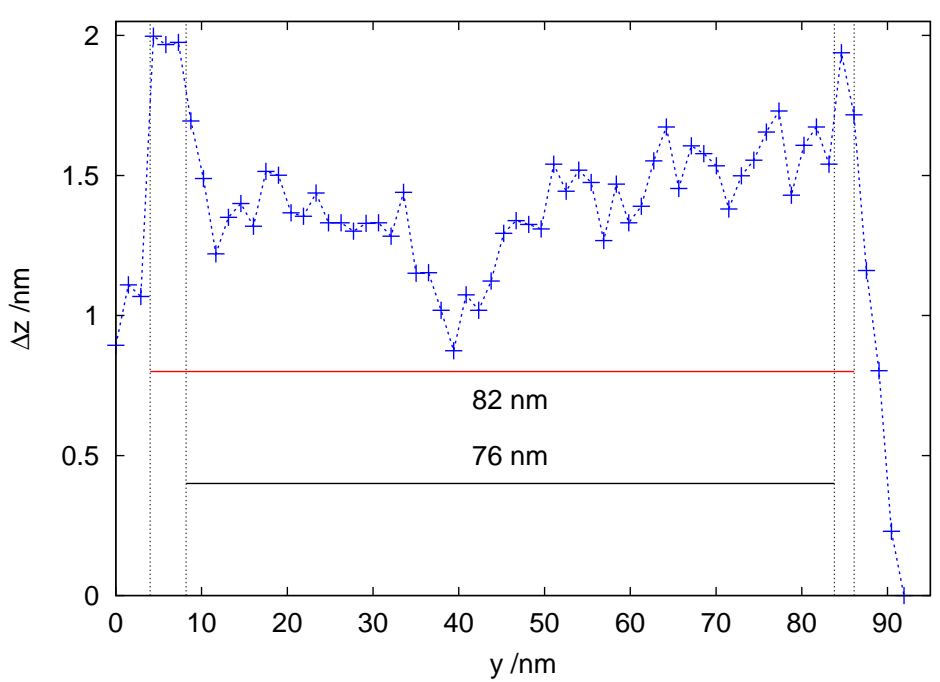

(a) Messung

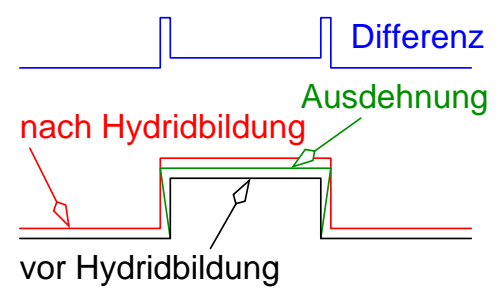

(b) Erläuterung

Abbildung 4.31: Höhenprofil des Differenzbildes aus Abb. 4.30(e) entlang der eingezeichneten Linie. Am Rand der Terrasse kommt es zu einer Erhöhung, da sich die Schicht bei der hier offenen Oberflächenmorphologie auch lateral ausdehnen kann. Die laterale Ausdehnung führt im Höhenprofil eines Differenzbilds zu einer Randerhöhung, dies ist in Skizze (b) verdeutlicht.

von Wasserstoff hat sich in einigen Bereichen Hydrid gebildet (Abb. 4.30(b)). Der Höhenunterschied zwischen $\alpha$-Phase und Hydrid ist nur sehr gering und beträgt ca. 1.1(3) nm. Dieser Messwert passt sehr gut zu dem in Abschnitt 3.3.2 (s. S. 42) berechneten Veränderung bei einer offenen Struktur von $1.1 \mathrm{~nm}$. Da sich neben der Schichtdicke auch die elektronische Struktur des Niobs verändert, könnte die gemessene Höhenänderung bei diesen geringen Variationen auch zu einem messbaren Teil durch eine Veränderung der Zustandsdichte verursacht sein. Eine veränderte Zustandsdichte der Oberfläche hat bei einem konstanten Strom auch einen anderen Spitzenabstand zur Folge. Diese Veränderung durch die elektronischen Eigenschaften liegt im Bereich von ca. $0.1 \mathrm{~nm}\left[\mathrm{PGB}^{+} 00\right]$ und kann hier zwar messbar in Erscheinung treten, aber der gemessene Wert liegt auch mit dieser Berücksichtigung im erwarteten Bereich.

Neben dieser zur Oberfläche senkrechten Ausdehnung kommt es auch zu einer Ausdehnung einzelner Terrassen parallel zur Substratoberfläche. Dies ist daran zu erkennen, dass die Ränder der Differenzbilder (Abb. 4.30(c), 4.30(d) und 4.30(e)) heller als der innere Bereich sind und sich damit diese Bereiche nach der Hydridbildung stärker als der Rest ausgedehnt haben. Die Randerhöhung ist noch einmal in Abb. 4.31 verdeutlicht, in der das Höhenprofil entlang der in Abb. 4.30(e) eingezeichneten Linie dargestellt ist. Es ist zu sehen, dass die Terrasse nach der Beladung eine Breite von 82(2) nm besitzt. Mit einer Ursprungsbreite von 76(2) nm beträgt die in-plane Vergrößerung 3(2) nm an beiden Rändern. 


\section{Diskussion}

Die in Kapitel 4 dargestellten experimentellen Resultate werden im Folgenden interpretiert und mit den in der Literatur beschriebenen Ergebnissen verglichen. In Abschnitt 5.1 wird zunächst die Wasserstoffaufnahme der $\alpha$-Phase behandelt. Danach werden in Abschnitt 5.2 die Hydridbildung und die beiden unterschiedlichen Ausscheidungstypen T1 und T2 genauer beschrieben. In Abschnitt 5.3 wird auf die zeitliche Entwicklung des Hydridanteils eingegangen.

\subsection{Wasserstoffaufnahme in der $\alpha$-Phase und Randlöslichkeit}

Im Abschnitt 4.1 (s. S. 45) sind die Messungen von Wasserstoffbeladungen der epitaktischen Niobschichten in der $\alpha$-Phase vorgestellt worden. Die Kinetik der Be- und Entladung mit Wasserstoff aus der Gasphase bei Raumtemperatur wird durch die Umwandlung der Wasserstoffmoleküle in den atomaren Wasserstoff bestimmt (s. Abschnitte 4.1.1 und 4.1.2, S. 47), wie dies von G. Hörz und E. Fromm angegeben wurde [HF76]. Da zum Zeitpunkt der Phasenumwandlung die Konzentration in der Probe bekannt ist, kann der Stickingfaktor $s$ bei der Beladung zu ca. 3\% bestimmt werden. Dieser Wert passt in den von L. Johnson et.al. angegebenen Bereich von 1\% bis 20\% [JDD72]. In der Arbeit von L. Johnson et.al. wurde der Stickingfaktor in Abhängigkeit vom Bedeckungsgrad einer Nioboberfläche mit Wasserstoff angegeben. Der in dieser Arbeit bestimmte Wert ist ein Durchschnittswert für einen Zeitraum von $210 \mathrm{~s}$ und entspricht dem Literaturwert.

Die Randlöslichkeit des Wasserstoffs wurde in Abschnitt 4.1.3 (s. S. 47) behandelt. Ab einem Druck von 6(1) $\cdot 10^{-7}$ mbar Wasserstoff beginnt die Bildung der Hydridphase. Bei den untersuchten Filmdicken wurde keine signifikante Schichtdickenabhängigkeit der Randlöslichkeit im Bereich von $20 \mathrm{~nm}$ bis $120 \mathrm{~nm}$ festgestellt*. Die Messungen sind bei einer Temperatur von $20^{\circ} \mathrm{C}$ durchgeführt worden. Bei dieser Temperatur liegt der Vergleichswert für massive Proben nach J. A. PRYDE und C. G. Тiтсомв [PT69] bei $1.9 \cdot 10^{-7}$ mbar (vgl. Abb. 2.5, S. 7). Der Vergleich der Druckwerte, bei dem die Hydridbildung stattfindet, ergibt, dass für dünne Schichten ein höherer Druck nötig ist als für massive Proben. Von G. Song et.al. wird

\footnotetext{
*Dagegen hat sich eine signifikante Schichtdickenabhängigkeit in der Randlöslichkeit bei elektrochemisch beladenen Proben ergeben, wie von M. Dornherm [Dor02] gezeigt wurde. Dies kann da durch erklärt werden, dass bei der hier durchgeführten Beladung aus der Gasphase die Konzentration in der Probe nicht explizit bestimmt wurde.
} 
berichtet, dass die kritische Temperatur der $\alpha$ - $\alpha^{\prime}$ Entmischung mit sinkender Schichtdicke abnimmt. Es wird geschrieben, dass ab einer Schichtdicke von weniger als $50 \mathrm{~nm}$ bei Zimmertemperatur keine Entmischung der beiden Phasen auftritt [SGAZ96]. Diese starke Verringerung der kritischen Temperatur in Abhängigkeit der Schichtdicke entspricht nicht den in dieser Arbeit erhaltenen Daten. Im Schichtdickenbereich von $20 \mathrm{~nm}$ bis $120 \mathrm{~nm}$ wurde bei Zimmertemperatur für jede gemessene Schichtdicke eine Entmischung von $\alpha$-Phase und Hydridphase beobachtet.

Durch das Beladen innerhalb der $\alpha$-Phase werden keine zusätzlichen Versetzungen gebildet. Es hat sich gezeigt, dass die Beladung in der $\alpha$-Phase reversibel ist (vgl. Abschnitt 4.1.4, S. 49). Bei einer Erniedrigung des Drucks verlässt der Wasserstoff die Schicht wieder, was anhand der abnehmenden mechanischen Spannung beobachtet werden kann (vgl. Abb. 4.1, S. 48). Die Oberflächenmorphologie verändert sich bei Beladung nicht.

\subsection{Hydridbildung}

Im Verlauf der Beladung wurden zwei unterschiedliche Typen von Hydridausscheidungen gebildet (s. Abschnitt 4.2.2, S. 56), die als Ausscheidungstypen T1 und T2 bezeichnet wurden. Die beiden Typen werden im Folgenden genauer erklärt, wobei u.a. auf die Berechnungen mit der Finite-Elemente-Methode für kohärente Ausscheidungen in dünnen Schichten eingegangen wird.

\subsubsection{Ausscheidungen vom Typ T1}

$\mathrm{Zu}$ Beginn der Beladung entstehen Typ T1-Ausscheidungen. Diese konnten als zylindrisch, in z-Richtung die Schicht durchdringend identifiziert werden. Diese Ausscheidungen weisen eine von der Schichtdicke abhängige maximale laterale Größe auf. Der Zusammenhang zwischen der Maximalgröße und der Filmdicke ist in Tabelle 4.3 (s. S. 57) zusammengestellt. Die Ausscheidungen werden bei dünneren Filmen etwas größer als in dickeren. Bei einer Filmdicke von $80 \mathrm{~nm}$ werden Ausscheidungsradien bis zu $35(2) \mathrm{nm}$ beobachtet und bei einem $40 \mathrm{~nm}$ dünnen Film betrug der Radius bis $\mathrm{zu} 40(2) \mathrm{nm}$. Eine Fläche von etwa $0.004(1 / 2) \mu \mathrm{m}^{2}$, was einem Radius von 36(3) nm entspricht, wird für Ausscheidungen bei einer Filmdicke von $70 \mathrm{~nm}$ beobachtet. In diesem Filmdickenbereich stimmt die Auftragung von Ausscheidungshöhe gegen Größe mit den Berechnungen für kohärente Ausscheidungen überein (vgl. Abb. 4.23, S. 73). Die experimentell gefundenen Ausscheidungen lassen sich daher als kohärent zur $\alpha$ Phase gebildet ansehen. Sie haben eine zylindrische Form, die in $z$-Richtung durch die komplette Schicht reicht. Nur zylinderförmige Ausscheidungen können die gemessenen Werte erklären, da Zylinder an der Oberfläche runde Veränderungen verursachen. Mit anderen Formen runden Querschnitts wird die gemessene vertikale Erhöhung der Oberfläche nicht erreicht, was beim Vergleich von berechneten Ausscheidungen der 


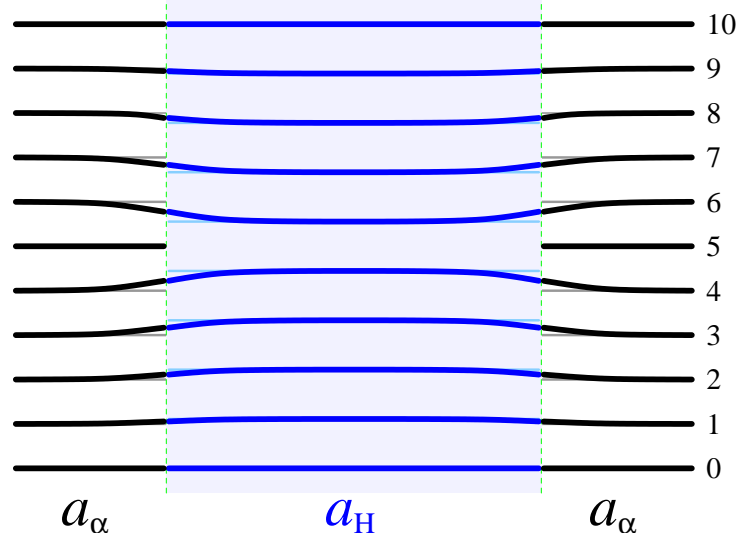

Abbildung 5.1: Querschnitt durch Ausscheidung und Matrix, die sich in der Gitterkonstante um 10\% unterscheiden. Der Einfluss des Verzerrungsfeldes erstreckt sich hier exemplarisch über zehn Ebenen $\left(\frac{a_{\alpha}}{a_{\mathrm{H}}-a_{\alpha}}=10\right)$. Die nullte und die zehnte Ebene sind nicht gekrümmt.

Form von Kugeln, Ellipsoiden und Zylindern mit den Messdaten in Abb. 4.23 (S. 73) gut zu erkennen ist.

Um die Schichtdickenabhängigkeit der lateralen Maximalgröße von kohärenten Typ T1-Ausscheidungen zu verstehen, wird die Selbstenergie eines Versetzungsrings bestimmt, der um eine zylinderförmige Ausscheidung verläuft. Diese Energie wird berechnet, da bei der Umwandlung vom kohärenten Typ T1 zum inkohärenten Typ T2 Versetzungen entstehen. Zur Berechnung wird die von F. KROUPA veröffentlichte Formel [Kro60]

$$
W(r) \approx \frac{E \cdot b^{2}}{2 \cdot(1-\nu)} \cdot r \cdot\left(\ln \frac{8 \cdot r}{\varrho}-1\right)
$$

herangezogen, die die elastische Energie eines zylinderförmigen Versetzungsrings in einer homogenen Matrix bestimmt. Dabei gibt $(E / 1-\nu)$ die elastischen Eigenschaften der Niobschicht, $b$ die Länge des Burgersvektors, $\varrho$ den Abschneideradius des Versetzungskerns $(\varrho \approx b / 3$ [STY89]) und $r$ den Radius des Versetzungsrings an. Mit dieser Formel wird die Energie des zylindersymmetrischen, unendlich ausgedehnten elastischen Versetzungsfeldes bestimmt. Bei der Integration hat F. KroupA nur den zylinderförmigen Innenraum berücksichtigt, da das Feld im Außenraum durch die Überlagerung der Fernfelder (der gegenüberliegenden Seiten) stark abfällt und vernachlässigt werden kann. Bei diesem Modell ist das Verzerrungsfeld in z-Richtung unendlich ausgedehnt, da das Modell von einer einheitlichen Gitterkonstante für den gesamten Raum ausgeht.

Für die Berechnung des hier vorliegenden Falls einer zylinderförmigen Ausscheidung, die eine andere Gitterkonstante $a_{\mathrm{H}}$ als die Matrix $a_{\alpha}$ besitzt, wird das Modell geringfügig modifiziert. Das Verzerrungsfeld ist in z-Richtung aufgrund der unterschiedlichen Gitterabstände begrenzt. In Abb. 5.1 ist exemplarisch ein Unterschied von $10 \%$ in der Gitterkonstante benutzt worden. Dann erstreckt sich das Versetzungsfeld über 10 Ebenen, da die nullte und die $(a / \Delta a)$ te Ebene keine Krümmung aufweisen sollte. Als erste Näherung wird hier angenommen, dass die Selbstenergie in diesem veränderten Modell mit der von F. KRoupA angegebenen Formel (5.1) berechnet werden kann und dass die Energie in einem Zylinder mit dem Radius $r$ und der Höhe 


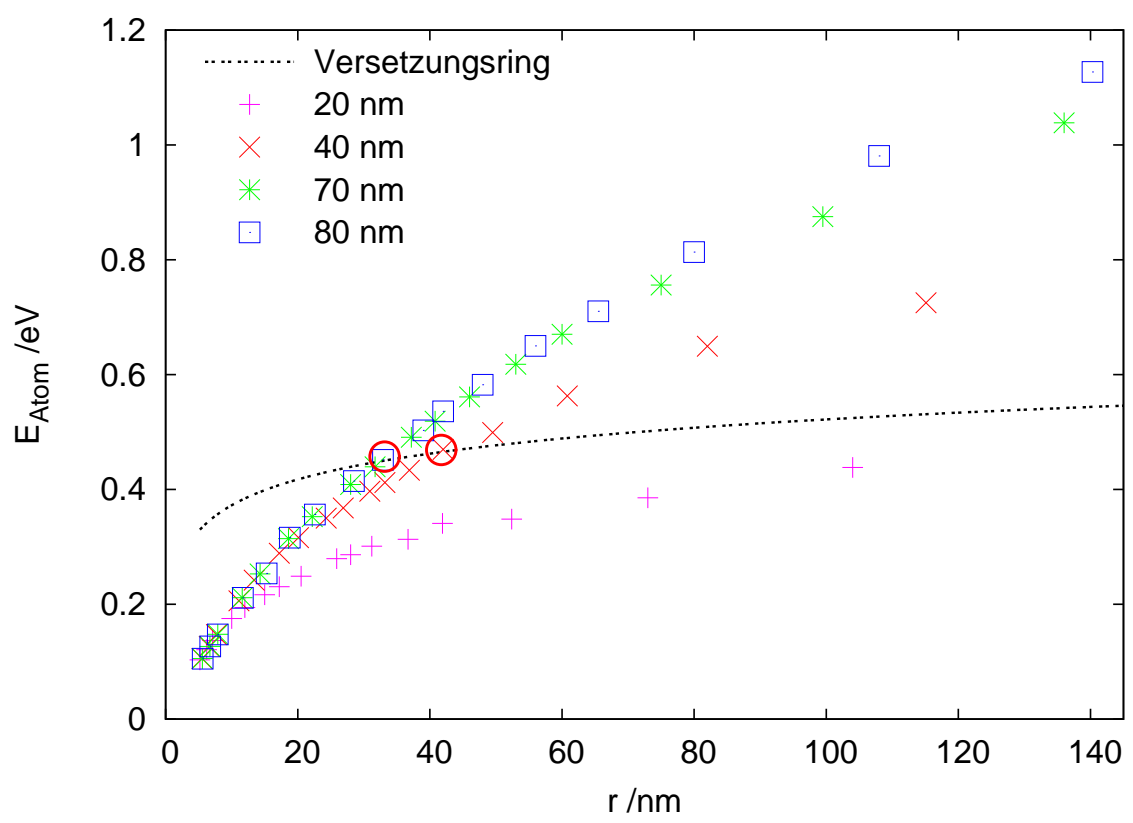

Abbildung 5.2: Elastische Energie, die in zylinderförmigen Ausscheidungen gespeichert ist (vgl. Abb. 3.22, S. 42). Hier ist zusätzlich noch die Selbstenergie eines Versetzungsrings eingezeichnet [Kro60]. Die Schnittpunkte dieser Kurve mit den Verläufen der elastischen Energie von Ausscheidungen für die unterschiedlichen Schichtdicken treten bei den Schichtdicken $40 \mathrm{~nm}, 70 \mathrm{~nm}$ und $80 \mathrm{~nm}$ ungefähr bei dem Radius auf, bei dem sich die Versetzungen bilden und die Umwandlung vom Typ T1 zum Typ T2 stattfindet. Bei der Schichtdicke von $20 \mathrm{~nm}$ wurde kein Schnittpunkt gefunden.

$h=a^{2} / \Delta a$ konzentriert ist. Die Selbstenergie eines Versetzungsrings kann dementsprechend auf die Anzahl der Oberflächenatome dieses Volumens normiert werden. Bei einem Unterschied zwischen den Gitterkonstanten der $\alpha$-Phase und des Hydrids von $2.9 \%$ ergibt sich eine Höhe des Zylinders von 35 Ebenen in $\langle 110\rangle$-Richtung. Diese so bestimmte Selbstenergie eines Versetzungsrings wird mit der radiusabhängigen elastischen Energie von zylinderförmigen Ausscheidungen verglichen, die in Abschnitt 3.3 (S. 35) bestimmt wurde. Der Verlauf der Energie in Abhängigkeit vom Radius ist in Abb. 5.2 dargestellt. Der Verlauf der Selbstenergie eines Versetzungsrings ist mit einer schwarzen Linie eingezeichnet und die elastische Energie von zylinderförmigen Ausscheidungen in Filmen ist mit unterschiedlichen Schichtdicken eingetragen. Die vier verschiedenen Schichtdicken sind mit farbigen Symbolen markiert.

Für kleine Radien ist die Selbstenergie eines Versetzungsrings größer als die in den zylinderförmigen Ausscheidungen vorhandene elastische Energie. Bei einem Radius von 33(2) nm entspricht die Selbstenergie des Versetzungsrings der elastischen Energie einer zylinderförmigen Ausscheidung in einem Film mit der Dicke von $70 \mathrm{~nm}$ bzw. $80 \mathrm{~nm}$. Dies ist mit einem roten Kreis im Graphen markiert (vgl. Abb. 5.2). Für eine Schichtdicke von $40 \mathrm{~nm}$ ist die Energie von Ausscheidung und Versetzungs- 
Tabelle 5.1: Vergleich der Maximalgröße von Typ T1-Ausscheidungen (vgl. Tab. 4.3, S. 57) und der Ausscheidungsgröße ab der sich Versetzungen bilden können. Typ T1Ausscheidungen werden bis zu derjenigen Größe beobachtet, bis zu der die elastische Energie ausreicht, um einen Versetzungsring zu bilden.

\begin{tabular}{|c|c|c|}
\hline $\begin{array}{c}\text { Schicht- } \\
\text { dicke }[\mathrm{nm}]\end{array}$ & $\begin{array}{c}\text { Maximalgröße Typ T1 } \\
{[\mathrm{nm}]}\end{array}$ & $\begin{array}{c}\text { Minimalgröße für } \\
\text { Versetzungsbildung [nm] }\end{array}$ \\
\hline 20 & nur Typ T1 & $\gg 300$ \\
\hline 40 & $\leq 40(2)$ & $43(2)$ \\
\hline 70 & $\leq 36(3)$ & $33(2)$ \\
\hline 80 & $\leq 35(2)$ & $33(2)$ \\
\hline
\end{tabular}

ring bei einem Radius von 43(2) nm identisch. Diese Werte stimmen mit den Radien überein, bei denen die Umwandlung vom Typ T1 zum Typ T2 stattfindet. Dies wird im Vergleich der lateralen Maximalgröße von Typ T1-Ausscheidungen und der Ausscheidungsgröße ab der sich Versetzungen bilden können (vgl. Tabelle 5.1) deutlich. Für eine Schichtdicke von $20 \mathrm{~nm}$ ist die elastische Energie einer Ausscheidung bis zu einem Radius von $300 \mathrm{~nm}$ kleiner als die Selbstenergie eines Versetzungsrings. Dies haben Berechnungen bis zu dieser Ausscheidungsgröße gezeigt. In der Messung wurden Ausscheidungen mit einer solchen Größe nicht beobachtet. Es existieren bei dieser Schichtdicke nur Typ T1-Ausscheidungen. Des Weiteren wurde gezeigt, dass sich die inkohärenten Typ T2-Ausscheidungen erst ab einer bestimmten lateralen Größe bilden können. Diese Umwandlungsgröße ist schichtdickenabhängig. Je dicker ein Film ist, um so kleinere inkohärente Ausscheidungen können beobachtet werden. Bei sehr dünnen Filmen wird die Ausscheidungsgröße, bei der eine Umwandlung möglich ist, im Experiment nicht erreicht, weshalb bei einer Filmdicke von $20 \mathrm{~nm}$ nur kohärente Ausscheidungen beobachtet wurden.

In Abschnitt 4.4 (s. S. 80) wurde ein $20 \mathrm{~nm}$ dünner Niobfilm beschrieben, dessen Oberfläche Mäanderstrukturen aufwies. Bei dieser Oberflächenmorphologie ist es möglich, dass bei der Hydridbildung auch eine Ausdehnung in der $x$ - $y$-Ebene auftritt (vgl. Abb. 4.31, S. 82). In dieser Messung ist eine Dehnung in $y$-Richtung von 3(2) nm bestimmt worden. In Abschnitt 3.3.2 (s. S. 42) ist die Berechnung mit der FiniteElemente-Methode für eine solche Drahtgeometrie vorgestellt worden. Der dort bestimmte Wert, von $1.1 \mathrm{~nm}$ Ausdehnung senkrecht zur Drahtrichtung, stimmt im Rahmen des Messfehlers mit dem experimentellen Wert überein. Ein Vergleich zwischen berechnetem und experimentell bestimmten Höhenprofil ist in Abb. 5.3 aufgetragen. Es ist zu erkennen, dass die experimentellen Daten sehr gut mit der Berechnung erklärt werden können. Beide Profile stimmen in der Breite überein. Sowohl bei der Messung als auch bei der Berechnung ist eine Höhenzunahme von $1.1 \mathrm{~nm}$ bestimmt worden. In den experimentellen Daten hat der Anstiegswinkel vom Gebiet der $\alpha$-Phase zur Hydridausscheidung 2.5(4) ${ }^{\circ}$ betragen. Bei der Berechnung wurde ein Anstiegswinkel von $2.8(1)^{\circ}$ bestimmt. 


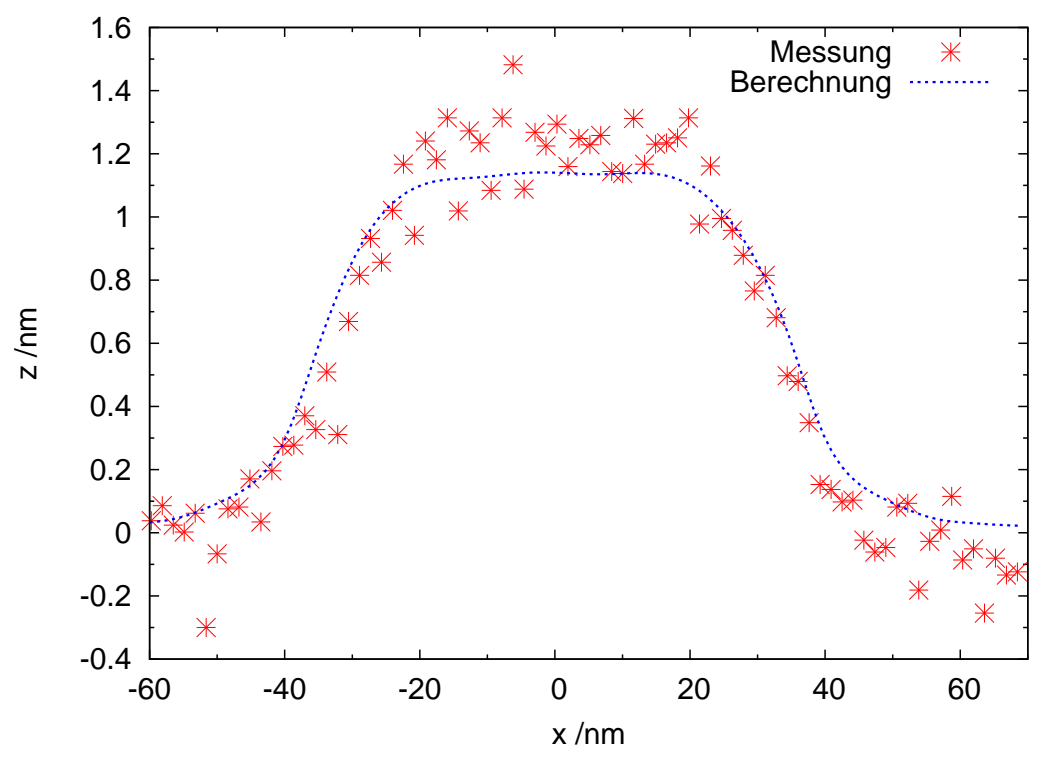

Abbildung 5.3: Vergleich eines Höhenprofils einer Ausscheidung in einer $20 \mathrm{~nm}$ dünnen Niobschicht parallel zur Mäanderstruktur der in Abb. 3.24 (s. S. 43) dargestellten Berechnung. Beide Profile stimmen in der Breite, der Höhe und dem Anstiegswinkel gut überein [PNS06].

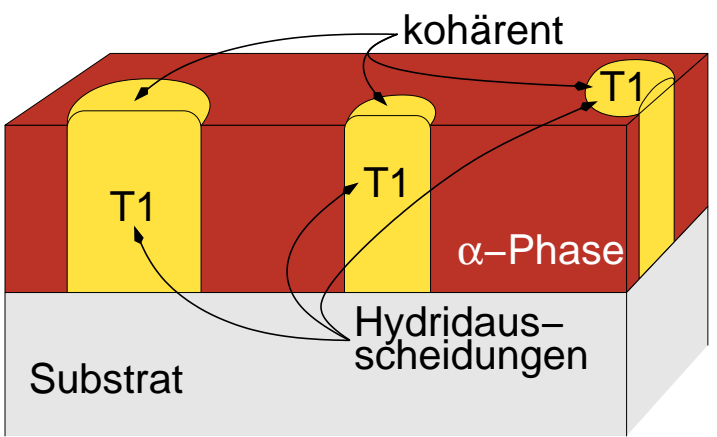

Abbildung 5.4: Schematischer Querschnitt von Typ T1-Ausscheidungen. Die Ausscheidungen sind Zylinder, die bis zum Substrat reichen und kohärent zur $\alpha$ Phase sind.

Für kurze Abstände $\xi$ treten bevorzugte Ausrichtung verschiedener Typ T1Ausscheidungen zueinander auf. Bei der mit dem Faktor $\xi^{-3}$ gewichteten Winkelverteilung in Abb. 4.13(b), S. 62 treten die elastisch weichen $\langle 111\rangle$-Richtungen bevorzugt auf. Dieser Befund stimmt mit den von H. Yamauchi und D. De Fontaine vorgestellten Berechnungen überein [YDF78]. Sie haben gezeigt, dass die Wechselwirkungsenergie zwischen zwei Defektagglomeraten ${ }^{\dagger}$ entlang der elastisch weichen Richtung attraktiv ist und mit dem Abstand wie $\xi^{-3}$ abfällt.

Eine schematische Zeichnung der Typ T1-Ausscheidungen in einer dünnen Schicht zeigt Abb. 5.4. Dort ist dargestellt, dass der Übergang von der $\alpha$-Phase zur Ausscheidung unter einem geringen Winkel stattfindet. Dieser Anstiegswinkel beträgt 2.8(3)

\footnotetext{
$\dagger$ In der Berechnung wurde die Wechselwirkung von zwei unterschiedlichen Veränderungen der Matrix behandelt. Dies können Agglomerationen von Punktdefekten oder aber Ausscheidungen sein.
} 


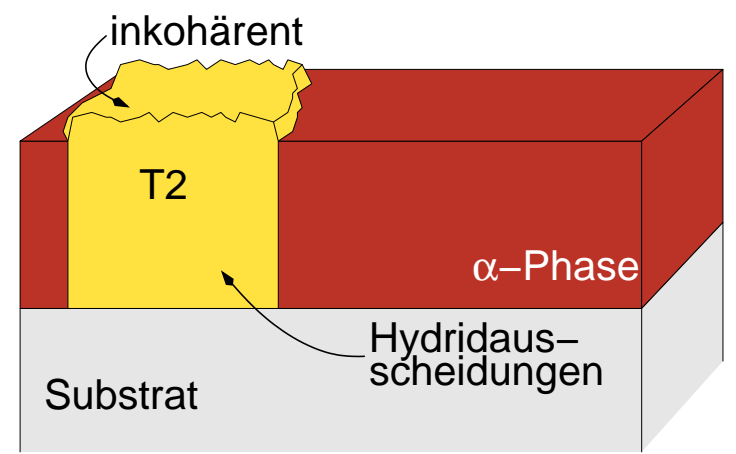

Abbildung 5.5: Schematischer Querschnitt einer Typ T2-Ausscheidung. Die Ausscheidung ist inkohärent zur $\alpha$-Phase. Die Begrenzung der Ausscheidung ist nicht regelmäßig und die Oberfläche sehr rau.

und ist unabhängig von der Schichtdicke (vgl. Tabelle 4.3, S. 57). Ebenso zeigt sich die Schichtdickenunabhängigkeit des Anstiegswinkels bei der in Abschnitt 3.3 (s. S. 35) beschriebenen Berechnung mit der Finite-Elemente-Methode, wo der Anstiegswinkel 3.1(1) ${ }^{\circ}$ beträgt. Das Messergebnis entspricht somit dem Wert, den die Berechnungen für kohärente Ausscheidungen voraussagen. Der Höhenunterschied von den größten Ausscheidungen zur $\alpha$-Phase zeigte keine messbare Schichtdickenabhängigkeit und beträgt 1.9(3) nm. Die Schichtdickenunabhängigkeit von Anstiegswinkel und Höhenveränderung ist dadurch zu erklären, dass bei kohärenten Ausscheidungen die in $z$-Richtung auftretenden Spannungen den Übergang von der $\alpha$-Phase zur Ausscheidung bestimmen. Die Oberflächenmorphologie der Typ T1-Ausscheidungen ändert sich gegenüber der $\alpha$-Phase nicht. Das ist ein weiteres Indiz für die Kohärenz zwischen $\alpha$-Phase und den Typ T1-Ausscheidungen.

\subsubsection{Ausscheidungen vom Typ T2}

Die großfächigen Typ T2-Ausscheidungen entstehen aus den Typ T1-Ausscheidungen. Die Umwandlung vollzieht sich durch die Bildung von Versetzungen. Eine schematische Zeichnung dieser Ausscheidung ist in Abb. 5.5 dargestellt. Diese Ausscheidungen sind inkohärent zur $\alpha$-Phase. Die Höhenänderung gegenüber der $\alpha$-Phase ist abhängig von der ursprünglichen Schichtdicke $h_{0}$ und beträgt $\Delta z=0.068 \cdot h_{0}$ (vgl. Abschnitt 4.2.1.6, S. 55). Die Ausscheidungen wachsen langgestreckt wie in den Abb. 4.5, 4.6 und 4.7 (s. S. 52 - 53) zu erkennen ist. Auf diesen STM-Bildern sind zwei bevorzugte Wachstumsrichtungen zu sehen, die zwei verschiedenen $\langle 111\rangle$-Richtungen der Niobschicht entsprechen. Beim Vergleich dieser Richtungen mit dem richtungsabhängigen E-Modul von Niob (s. Abb. 2.8, S. 12) wird festgestellt, dass das Hydrid bevorzugt entlang der elastisch weichen $\langle 111\rangle$-Richtungen des Niobs wächst, da die Ausscheidungen das sie umgebene Material verformen. Bei gleicher Verformung $\epsilon$ ist in den elastisch weichen $\langle 111\rangle$-Richtungen eine geringere Spannung $\sigma$ notwendig. Somit sind die elastisch weichen Richtungen bevorzugte Wachstumsrichtungen.

Auf der Oberfläche der Typ T2-Ausscheidungen werden viele Gleitstufen beobachtet. Eine solche Oberfläche ist in Abb. 4.16 auf Seite 65 dargestellt. Die Gleitstufen treten entlang der $\langle 1 \overline{1} 1\rangle$ - und $\langle\overline{1} 13\rangle$-Richtung auf. Diese Stufenrichtungen entstehen, wenn die Versetzungen auf den (1 $\overline{2} 1)$ - und (011)-Ebenen zur (110)-Oberfläche 


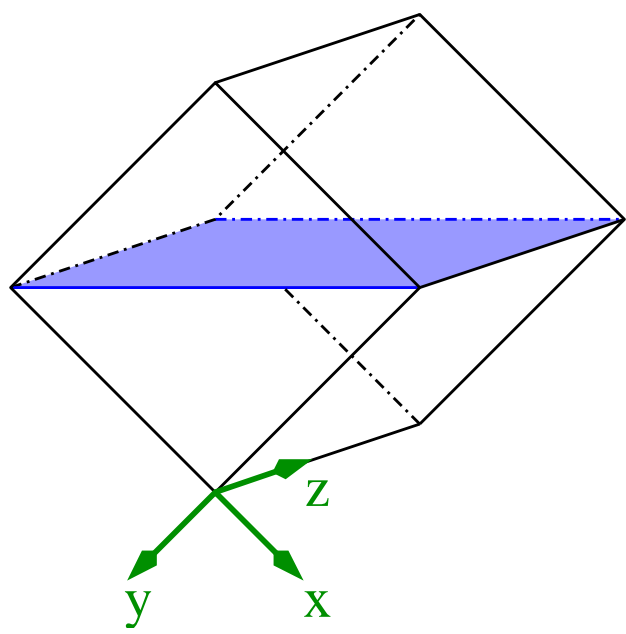

(a) Einheitszelle

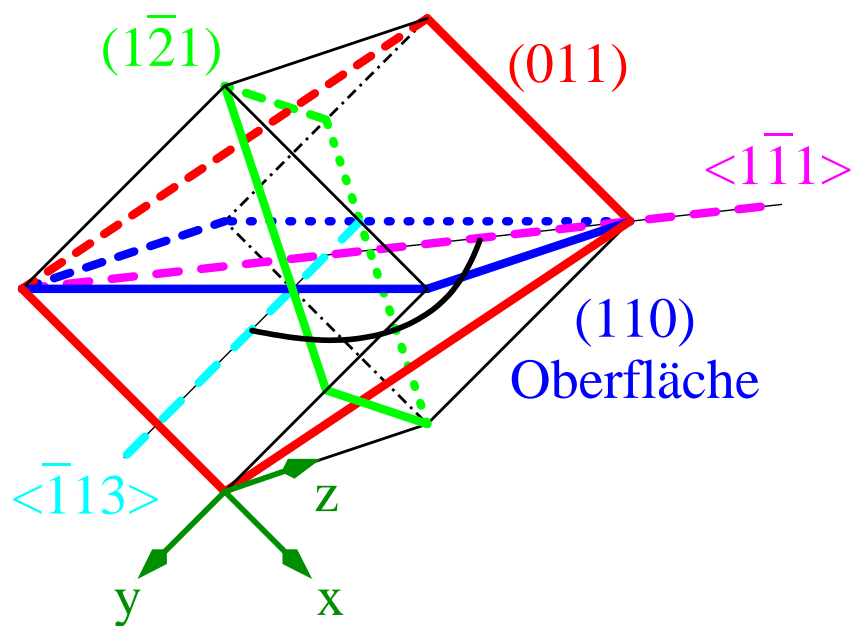

(b) Gleitebenen

Abbildung 5.6: Bevorzugtes Gleitsystem, eingezeichnet in die Einheitszelle. In (a) ist die Einheitszelle so gezeichnet, dass die (110)-Ebene parallel zur Oberfläche liegt. In (b)

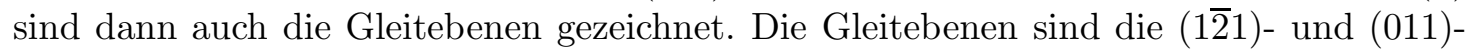
Ebenen. Die Schnittlinie der Oberfläche und der (011)-Ebene ist die $\langle 1 \overline{1} 1\rangle$-Richtung. In der $\langle\overline{1} 13\rangle$-Richtung trifft die (151)-Ebene mit der Oberfläche zusammen. In diese beiden Richtungen sind die in Abb. 4.16 erkennbaren Gleitstufen orientiert.

transportiert werden. Zur Veranschaulichung dieser Ebenen und Richtungen ist in Abb. 5.6(a) eine Einheitszelle so eingezeichnet, dass die (110)-Ebene parallel zur Oberfläche liegt (entsprechend der Orientierung des Films). In Abb 5.6(b) sind dann zusätzlich die beiden Gleitebenen (12̄1) und (011) abgebildet. Die Gleitstufen entstehen durch das Schneiden der Gleitebenen mit der Oberfläche. Die beiden resultierenden Schnittlinien entstehen entlang der $\langle 1 \overline{1} 1\rangle$ - und $\langle\overline{1} 13\rangle$-Richtung und sind auch in der Abbildung dargestellt. Zwischen diesen beiden Richtungen der Gleitstufen tritt ein Winkel von $100^{\circ}$ auf, was der Differenz zwischen den beiden Maxima in der Winkelverteilung der Gleitstufen in Abb. 4.16(b), S. 65 entspricht.

\subsubsection{Randbereich zwischen $\alpha$-Phase und T2 Ausscheidungen}

Die Versetzungen, die im Hydrid entstehen, reichen bis in das Gebiet der $\alpha$-Phase hinein (s. Abschnitt 4.2.7, S. 66). Durch diese Versetzungen werden in-plane Spannungen abgebaut. In-plane Spannungen in den Gebieten der $\alpha$-Phase entstehen durch eine größere Gitterkonstante des Hydrids gegenüber der $\alpha$-Phase. Eine Folge ist, dass die Hydridbereiche die Gebiete der $\alpha$-Phase zusammendrücken. Die an der Oberfläche erzeugten Gleitstufen sind in $\langle 1 \overline{1} 1\rangle$ - und $\langle\overline{1} 13\rangle$-Richtung orientiert. In der Winkelverteilung der Stufenrichtung wird deutlich, dass die $\langle\overline{1} 13\rangle$-Richtung im gewählten Ausschnitt häufiger auftritt (s. Abb. 4.19, S. 69). Die Versetzungen wandern bevor- 


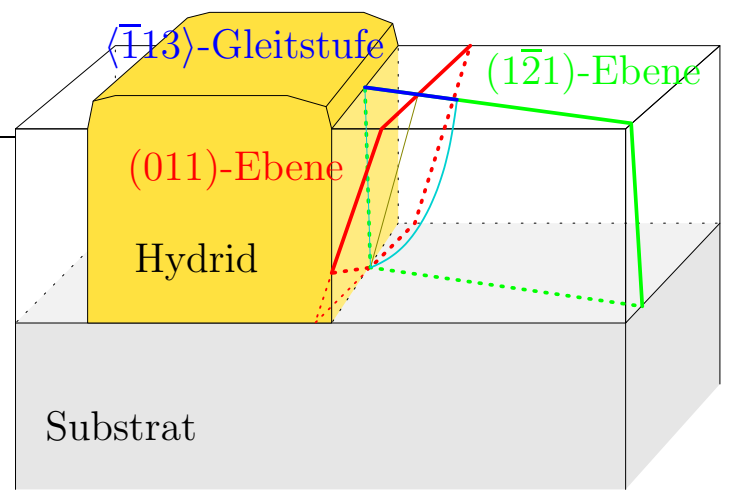

Abbildung 5.7: Entstehung von Gleitstufen im Randbereich der $\alpha$-Phase. Durch die Bildung des Hydrids entstehen in der $\alpha$-Phase mechanische Spannungen, die durch Versetzungen abgebaut werden. Beim Wandern der Versetzung entlang der (12̄1)-Ebene entsteht an der Oberfläche entlang der $\langle\overline{1} 13\rangle$-Richtung eine Stufe.

zugt entlang der (12̄1)-Ebene zur Oberfläche. Eine schematische Zeichnung dieser Ebenen ist in Abb. 5.7 dargestellt. An der Oberfläche ist eine Stufe entlang der blau dargestellten $\langle\overline{1} 13\rangle$-Richtung sichtbar. Diese entsteht, wenn die in cyan eingezeichnete Versetzungslinie entlang der grünen (12̄1)-Ebene wandert. In $\langle 1 \overline{1} 1\rangle$-Richtung orientierte Stufen entstehen durch Gleiten der Versetzungen in der (011)-Ebene. Bei den Positionen der Gleitstufen in Abb. 4.17(b) (s. S. 67) ist zu erkennen, dass der Winkel zwischen den Gleitstufen und der Grenze zwischen $\alpha$-Phase und Hydrid stets nahe $90^{\circ}$ ist. Auf welcher Gleitebene die meiste Spannung abgebaut wird, legt die Richtung der Gleitstufen in der $\alpha$-Phase fest (maximaler Schmidfaktor). Dies ist in dem beobachteten Ausschnitt bevorzugt die $\langle\overline{1} 13\rangle$-Richtung, die auf weiten Strecken fast senkrecht zur Grenze zwischen der $\alpha$-Phase und dem Hydrid liegt. An den Positionen, an denen die Grenzfläche anderes orientiert ist, werden auch Stufen entlang der $\langle 1 \overline{1} 1\rangle$-Richtung beobachtet.

\subsubsection{Einflussparameter auf die unterschiedlichen Ausscheidungstypen}

In Abschnitt 4.2.3 (s. S. 59) wurde gezeigt, dass die beiden unterschiedlichen Ausscheidungstypen nicht bei jeder Schicht aufgetreten sind. Es wurde untersucht, bei welchen Schichteigenschaften die beiden Ausscheidungstypen beobachtet werden. Dabei ist die Schichtdicke, die Oberflächenmorphologie und der Substratmiscut $\vartheta$ berücksichtigt worden. In Abb. 5.8 sind Filme mit verschiedenen Schichtdicken und unterschiedlichen Fehlschnitten des Substrates zusammengestellt. Dies ist ein Ausschnitt der Daten, die in Tabelle 4.4 (s. S. 59) zusammengetragen sind. Es wurde der Ausschnitt gewählt, in dem sich das Auftreten der beiden unterschiedlichen Ausscheidungstypen verändert. Die Darstellung zeigt, dass bei dickeren Filmen mit einem Substratmiscut von $\vartheta<0.1^{\circ}$ nur großflächige Typ T2-Ausscheidungen zu sehen sind. Bei geringeren Schichtdicken tritt dann auch der Typ T1 auf. Im Übergangsbereich, der für einen Miscut von $\vartheta<0.1^{\circ}$ bei ca. $40 \mathrm{~nm}$ Filmdicke liegt, sind beide Typen zu beobachten. Wird der Fehlschnitt des Substrates auf $\vartheta<0.2^{\circ}$ erhöht, verschiebt sich der Übergangsbereich zu einer Filmdicke von ca. $70 \mathrm{~nm}$. 


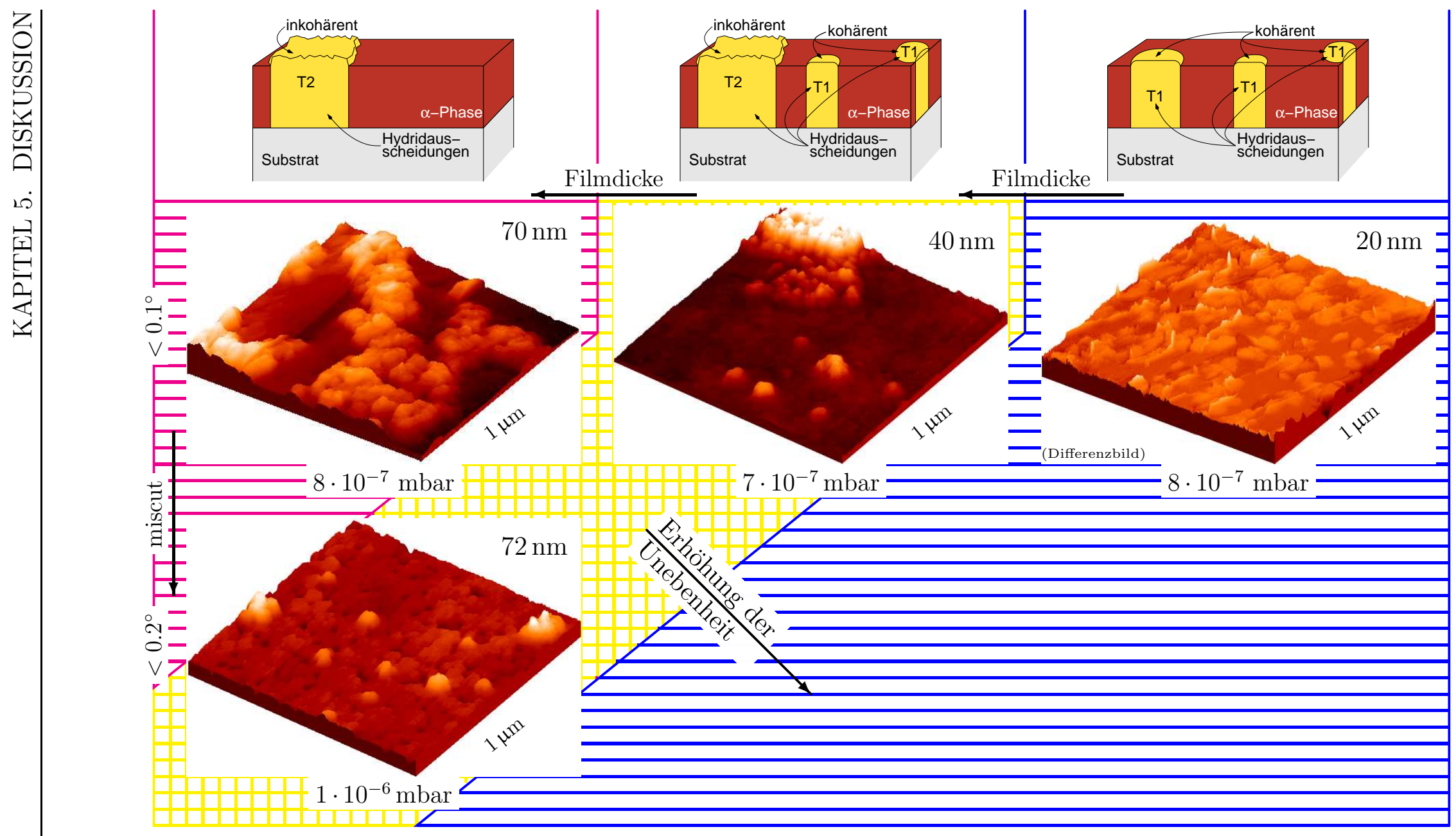

Abbildung 5.8: Unterschiedliche Ausscheidungstypen bei der Hydridbildung für verschiedene Schichteigenschaften. Bei dickeren Filmen ist die Bildung von wenigen großen Typ T2-Ausscheidungen bevorzugt. Bei dünnen Schichten ist der Typ T1 bevorzugt. Bei mittleren Schichtdicken bilden sich beide Typen. Die Schichtdicke des Übergangsbereiches hängt von der Schichtrauigkeit ab. Diese wird vom Substratmiscut beeinflusst. 
Die Inselhöhe und der RMS-Wert auf den Terrassen wird sowohl durch einen höheren Substratmiscut als auch durch die Verringerung der Schichtdicke erhöht (vgl. Abb 3.11, S. 28). Die Oberflächenmorphologie bestimmt daher die Bildung der beiden Ausscheidungstypen. Bei sehr großer Inselhöhe und geringer Schichtdicke, wie in der $20 \mathrm{~nm}$ Schicht, treten nur Typ T1-Ausscheidungen auf. Wegen der großen Höhenunterschiede wird bei dieser Schicht ein Differenzbild von vor und nach der Hydridbildung gezeigt. Wegen der geringen Schichtdicke reicht die durch die elastische Verformung gespeicherte Energie nicht aus, um Versetzungen zu bilden. In Abb. 5.2 (s. S. 86) ist zu erkennen, dass die elastische Energie der zylinderförmigen Ausscheidung in einem $20 \mathrm{~nm}$ dünnen Film geringer ist als die Energie eines Versetzungsrings. Dieses ist für den im Experiment beobachteten Größenbereich von Ausscheidung gültig. Folglich sind aus energetischen Gründen alle Ausscheidungen vom Typ T1. Bei größeren Schichtdicken entsteht die zur Versetzungsbildung benötigte Energie bereits bei geringeren Ausscheidungsgrößen. Ab einer Schichtdicke von $40 \mathrm{~nm}$ treten auch Typ T2-Ausscheidungen auf. Mit abnehmender Oberflächenunebenheit verringert sich die Anzahl der Ausscheidungen. Damit wird jede einzelne Ausscheidung größer. Der Typ T2 ist bei großen Ausscheidungen aufgrund der nicht vorhandenen elastischen Energie gegenüber den stark verspannten kohärenten Ausscheidungen bevorzugt. Deshalb wurden bei den dickeren Filmen nur Typ T2-Ausscheidungen beobachtet.

Die Flächendichte der auf der Nioboberfläche vorhandenen kleinen Palladiuminseln ist nicht ausschlaggebend für die Anzahl der Keimbildungszentren, da die Flächendichte der Palladiuminseln ca. $2 \cdot 10^{12} \mathrm{~cm}^{-2}$ beträgt (vgl. Abb. 3.12, S. 29). Sie ist somit deutlich größer als die Anzahl der Hydridausscheidungen mit weniger als $2 \cdot 10^{7} \mathrm{~cm}^{-2}$ (vgl. Abb. 4.22(a), S. 72). Des Weiteren ist die Inseldichte bei den unterschiedlichen Schichten nicht verändert worden, so dass sie nicht die Veränderung der Bildung von Ausscheidungstypen erklären kann.

Die Versetzungsdichte liegt bei ca. $7 \cdot 10^{10} \mathrm{~cm}^{-2}$ (vgl. Gl. (3.3), S. 26) und zeigt keine merkliche Schichtdickenabhängigkeit. Damit kann sie nicht zur Klärung der Veränderung bei der Bildung der Ausscheidungstypen herangezogen werden.

Mit der Unebenheit der Schicht verändert sich die Anzahldichte der Terrassenränder. Beim Übergang zu dünneren Schichten und größerem Substratmiscut wächst die Dichte der Terrassenränder. Die Anzahl der Keimbildungszentren verändert sich somit entsprechend der Dichte von den Terrassenränder. Die Häufigkeit der Terrassenränder erklärt somit die Veränderung bei der Bildung der Ausscheidungstypen.

\subsection{Keimbildung und Wachstum}

Die Wachstumsrate des Hydridanteils $X(t)$ ist in den Messungen durch zwei unterschiedliche Gesetze angepasst worden. Zum einen durch ein Potenzgesetz der Form $X(t)=(t / \tau)^{n}$ und zum anderen mit einer Johnson-Mehl-Avrami Kinetik, die die 
Zeitabhängigkeit $X(t)=j \cdot\left[1-\exp \left\{-\left(t / \tau^{\prime}\right)^{n}\right\}\right]$ besitzt. Das Potenzgesetz ist nur dann anwendbar, wenn sich die Einzugsgebiete der unterschiedlichen Ausscheidungen noch nicht überlappen. Nach P. HAASEN ist dies bis zu einem Anteil von $X(t)<0.2$ gegeben [Haa96]. Wenn die einzelnen Ausscheidungen nicht mehr unabhängig voneinander sind, wird das Wachstum durch eine Johnson-Mehl-Avrami Kinetik beschrieben. Der Übergang von der Johnson-Mehl-Avrami Kinetik zum Potenzgesetz kann als Näherung der Exponentialfunktion für kleine Werte von $\left\{t / \tau^{\prime}\right\}^{n}$ angesehen werden $(\exp \{x\} \approx 1+x)$. Dabei gehen die beiden unabhängigen Parameter $j$ und $\tau^{\prime}$ der Johnson-Mehl-Avrami Kinetik in einen Parameter $\tau$ des Potenzgesetzes über. Die Zeiten $\tau$ und $\tau^{\prime}$ aus den unterschiedlichen Anpassungen der Wachstumsrate lassen sich nicht miteinander vergleichen. Durch den Exponenten $n$ in beiden Gleichungen wird der vorhandene Wachstumsmodus charakterisiert [Chr65, Haa96]. Der Vorfaktor $j$ der Johnson-Mehl-Avrami Kinetik entspricht dem Anteil, der sich im thermodynamischen Gleichgewicht $(t \rightarrow \infty)$ einstellt.

\subsubsection{Anfangsstadien}

In Abschnitt 4.3.1 (s. S. 69) ist die zeitliche Entwicklung des Hydridanteils einer $70 \mathrm{~nm}$ dünnen Niobschicht beschrieben worden. Es ist gezeigt worden, dass der Hydridanteil über die gesamte Messung unterhalb von 20\% lag und das Wachstum des ausgeschiedenen Anteils mit einem Potenzgesetz beschrieben werden kann. Bei dieser Messung wurde das Wachstum des Hydridanteils durch die Formel

$$
X(t)=\left(\frac{t}{367(55) \min }\right)^{1.36(7)}
$$

beschrieben.

Der Exponent für das Wachstum des Hydridanteils von $n=1.36(7)$ liegt zwischen dem in der Literatur angegebenen Wert für das Wachsen von Zylindern $\left(t^{1}\right)$ und dem für Kugeln $\left(t^{3 / 2}\right)$ in massiven Proben. Für das Wachsen von Platten wird ein Verlauf von $\left(t^{2}\right)$ in drei Dimensionen angegeben (s. P. HAASEN [Haa96]). Da es sich bei den Ausscheidungen um Zylinder handelt, die in z-Richtung durch die komplette Schicht reichen, wird ein Verhalten wie bei Platten erwartet. Der Unterschied zu den gemessenen Exponenten ist dadurch zu erklären, dass bei den hier durchgeführten Messungen in dünnen Schichten die Diffusion von Wasserstoffatomen nur in der Ebene und nicht im Volumen möglich ist.

\subsubsection{Sättigung}

Die Sättigung der Ausscheidungsbildung wird erreicht, wenn die Probe über einen langen Zeitraum bei einem konstanten Druck gehalten wird und sich damit die Einzugsgebiete unterschiedlicher Ausscheidungen überlagern. Wie in Abschnitt 4.3.3 (s. S. 75) beschrieben wurde, wurde ein $30 \mathrm{~nm}$ dünner Niobfilm für drei Stunden bei 
einem Wasserstoffdruck von $7 \cdot 10^{-7}$ mbar gehalten. In Abb. 4.27 (s. S. 77) ist zu erkennen, dass in diesem Beladungszeitraum das Wachstum des Ausscheidungsanteils durch eine Johnson-Mehl-Avrami Kinetik der Form

$$
X(t)=0.45(4) \cdot\left\{1-\exp \left[-\left(\frac{t}{82(9) \min ^{\ddagger}}\right)^{1.36(10)}\right]\right\}
$$

angepasst wird. Der für die Johnson-Mehl-Avrami Kinetik berechnete Exponent von $n=1.36(10)$ stimmt sehr gut mit dem für die $70 \mathrm{~nm}$ dünne Niobschicht in Abschnitt 5.3.1 mit Hilfe eines Potenzgesetzes bestimmten Exponenten überein. Hiermit wird der Übergang von sich nicht beeinflussenden Einzugsgebieten zu sich überlagernden deutlich. Die beiden Zeitkonstanten $\tau$ lassen sich aufgrund der unterschiedlichen Zeitgesetze nicht miteinander vergleichen. Aus dem Vorfaktor $j=0.45(4)$ in Gl. (5.3), der den Volumenanteil bei $(t \rightarrow \infty)$ wiederspiegelt, lässt sich ablesen, dass sich bei diesem Film, der bei einem konstanten Druck beladen worden ist, ein Gleichgewicht zwischen $\alpha$-Phase und Hydrid einstellt.

Abschnitt 4.3 .4 (s. S. 78) beschreibt die Messung eines $80 \mathrm{~nm}$ dünnen Niobfilms. Dieser Film ist für eine Woche bei einem Druck von $9 \cdot 10^{-7}$ mbar beladen worden. Das Wachstum des Hydridanteils lässt sich bei dieser Messung durch die Gleichung

$$
X(t)=0.514(3) \cdot\left\{1-\exp \left[-\left(\frac{t}{64.4(5) \mathrm{h}^{\ddagger}}\right)^{1.50(2)}\right]\right\}
$$

beschreiben. Der Gleichgewichtswert $j=0.514(3)$ bleibt für diese Messung kleiner als eins, so dass sich in diesem Film bei konstantem äußeren Wasserstoffdruck und kontinuierlicher Beladung ein Gleichgewicht zwischen $\alpha$-Phase und Hydrid einstellt. Diese beiden Messungen geben sehr klare Hinweise dafür, dass in dünnen Schichten bei konstanten chemischen Potential und kontinuierlichem Wasserstoffangebot zwei unterschiedliche Phasen koexistieren können. Dieses zeigt sich nicht in massiven Proben (vgl. die Messungen von J. A. Pryde und C. G. Titcomb an massiven Niobproben [PT69]). Dieses veränderte Verhalten ist in Abb. 5.9 skizziert. In dünnen Schichten existiert kein Plateaudruck bei dem die Probe eine vollständige Phasenumwandlung vollzieht, wenn ausreichend Wasserstoff vorhanden ist (sehr lange Beladungszeit). Zur kompletten Phasenumwandlung in dünnen Schichten ist eine Druckerhöhung notwendig. Das chemische Potential steigt streng monoton mit der Wasserstoffkonzentration an (vgl. Abb. 2.7, S. 9).

Der ermittelte Avrami-Exponent von $1.3<n<1.5$ ist vergleichbar mit den in der Literatur angegebenen Werten. Z. MA und H. ALLEN finden bei Untersuchungen zur Phasenumwandlung von dünnen Ti Schichten (Dicke: $25 \mathrm{~nm}$ bis $100 \mathrm{~nm}$ ) auf Siliziumsubstraten zu TiSi 2 Schichten Exponenten zwischen 1.16(12) und 2.13(15) [MA94].

$\ddagger$ Die beiden unterschiedlichen Zeitkonstanten erklären sich durch die Vorbehandlung der Proben. Die $80 \mathrm{~nm}$ dünne Schicht wurde im Gegensatz zu der $30 \mathrm{~nm}$ dünnen Schicht nicht direkt nach der Herstellung mit Wasserstoff beladen. 


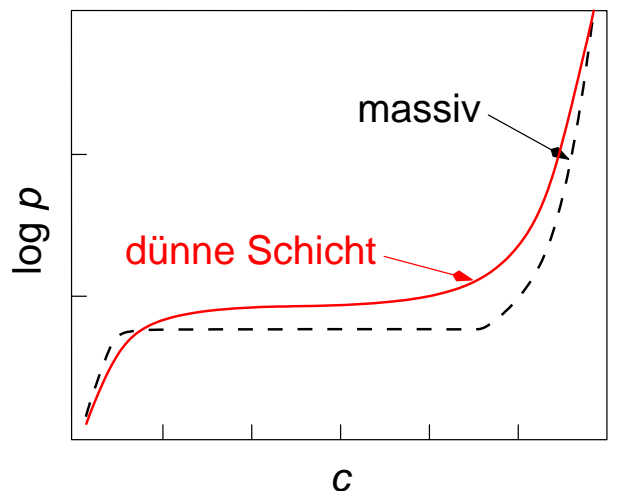

Abbildung 5.9: Schematische Darstellung der Veränderung einer Isotherme beim Übergang zu dünnen Schichten. In dünnen Schichten tritt kein Plateau auf, bei dem die Probe komplett in die andere Phase umgewandelt wird, wenn genügend Wasserstoff angeboten wird. Zur vollständigen Phasenumwandlung muss in dünnen Schichten der Druck erhöht werden. Eine längere Beladungszeit bei konstantem Druck wie bei massiven Proben reicht nicht aus.

Bei der Umwandlung von 1-3 $\mu$ m dicken amorphen $\mathrm{Al}_{78} \mathrm{~W}_{22}$ Schichten in den kristallinen Zustand wurden von T. CAR et.al. Exponenten im Bereich von 0.87(1) bis 1.6(2) gemessen [CRIT05]. C. Michaelsen el.al. geben für die Phasenumwandlung in $\mathrm{Ti} / \mathrm{Al}$ Multischichten $(\Lambda=72 \mathrm{~nm})$ einen Exponenten von 1.3 an [MBW97].

Die Zusammenstellung über verschiedene Avrami-Exponenten von J. W. CHRISTIAN [Chr65, Seite 489] zeigt, dass die hier bestimmten Werte für den Fall zutreffen, in dem das Wachstum diffusionskontrolliert ist und die Nukleationsrate abnimmt. Die sinkende Nukleationsrate ist in den Messungen zu erkennen. Bei der Messung der Anfangsstadien (s. Abb. 4.22(a), S. 72) ist zu sehen, dass die Nukleationsrate $\left(\mathrm{d} N / \mathrm{d} t \sim t^{-0.4}\right)$ mit zunehmender Zeit geringer wird. Die Messung einer $80 \mathrm{~nm}$ dünnen Niobschicht zeigt, dass der Anteil der Typ T1-Ausscheidungen nach 2.5 Tagen bei einem Wert von $7.5 \%$ stagniert (s. Abb. 4.29, S. 80). Die Nukleationskeime sind Typ T1-Ausscheidungen und somit wurde gezeigt, dass die Nukleationsrate absinken muss. Die gemessenen Parameter der Johnson-Mehl-Avrami Kinetik passen zu den in der Literatur veröffentlichten Werten.

Es wurde gezeigt, dass es möglich ist, mit der oberflächensensitiven Methode STM, die im Volumen stattfindende Ausscheidungsbildung zu beobachten. Durch kontinuierliche Messung von Topographiebildern ist es möglich, die Ausscheidungskinetik zu untersuchen. Es hat sich gezeigt, dass in dünnen Schichten zwei unterschiedliche Phasen im thermodynamischen Gleichgewicht nebeneinander existieren können. Bei den STM-Messungen sind kohärente und inkohärente Ausscheidungen beobachtet worden. Es kann festgehalten werden, dass unterhalb einer kritischen Schichtdicke keine inkohärenten Ausscheidungen gebildet werden können. 


\section{Zusammenfassung}

In der vorliegenden Arbeit ist die Wasserstoffaufnahme und die Hydridbildung in dünnen epitaktischen Niobschichten mit Hilfe der oberflächensensitiven Rastertunnelmikroskopie (STM) untersucht worden. Es wurde gezeigt, dass die Entstehung von Hydridausscheidungen in der Schicht aufgrund der unterschiedlichen Gitterparameter von Matrix und Ausscheidungen direkt beobachtet werden kann. Durch STMMessungen während der Beladung mit Wasserstoff aus der Gasphase ist es möglich, die zeitliche Entwicklung von Keimbildung und Wachstum zu bestimmen, sowie die Morphologie der Ausscheidungen zu untersuchen.

Die Wasserstoffaufnahme aus der Gasphase von dünnen Niobschichten wird durch die Kinetik an der Oberfläche festgelegt. Der dominante Einfluss für die Kinetik ist der eingestellte Wasserstoffpartialdruck. Die Geschwindigkeit konnte durch die mechanische Spannung und der daraus resultierenden Substratkrümmung (vgl. Abschnitt 3.2.4, S. 30) bestimmt werden. Die Beladung in der $\alpha$-Phase bis zur Hydridbildung hat einen Stickingfaktor von 3\% ergeben (vgl. Abschnitt 4.1.1, S. 45). Die Entladung des Wasserstoffs geschieht ungefähr mit der gleichen Geschwindigkeit wie die Beladung. Der zeitliche Verlauf ist durch einen Faktor von $\left(1-\exp \left\{-6 \cdot 10^{-3} \cdot t / \mathrm{s}\right\}\right)$ bestimmt. (vgl. Abschnitt 4.1.2, S. 47). Innerhalb der $\alpha$-Phase ist die Wasserstoffbeladung vollständig reversibel. Bei der Beladung in der $\alpha$-Phase verändert sich die Schichtoberfläche nicht. Die Oberflächenmorphologie der $\alpha$-Phase ist mit einer unbeladenen Probe vergleichbar (vgl. Abschnitt 4.1.4, S. 49).

Ab einem Wasserstoffdruck von $6(1) \cdot 10^{-7}$ mbar wird die Niobhydridphase gebildet. Der Beginn des Zweiphasengebiets zeigte im Bereich von $30 \mathrm{~nm}$ bis $120 \mathrm{~nm}$ keine Schichtdickenabhängigkeit (vgl. Abschnitt 4.1.3, S. 47).

Bei der Bildung der Hydridphase wurden zwei unterschiedliche Ausscheidungstypen gefunden, die sich grundlegend voneinander unterscheiden: Der Typ T1, der kohärent zur $\alpha$-Phase ist, und der inkohärente Typ T2.

Die kohärenten Ausscheidungen haben die gleiche Oberflächenmorphologie wie die sie umgebende $\alpha$-Phase. Der Höhenunterschied durch die lokale Gitterdehnung in der Ausscheidung zu den Gebieten der $\alpha$-Phase beträgt 1.9(3) nm. Diese Höhendifferenz wird mit einem langsamen Anstieg unter dem Winkel 2.8(3) erreicht. Die Höhendifferenz und der Anstiegswinkel sind unabhängig von der Schichtdicke (vgl. Tab. 4.3, S. 57). Die verschiedenen Typ T1-Ausscheidungen sind auf kurzen Entfernungen relativ zueinander entlang der elastisch weichen $\langle 111\rangle$-Richtungen des Niobfilms orientiert (vgl. Abb. 4.13, S. 62). Die Maximalgröße der kohärenten Ausscheidungen ist von der Schichtdicke abhängig. Bei einer Schichtdicke von $40 \mathrm{~nm}$ treten kohärente Ausscheidungen bis zu einem Radius von 40(2) nm auf. Im $80 \mathrm{~nm}$ dünnen 
Niobfilm sind keine Typ T1-Ausscheidungen mit einem Radius größer als 35(2) nm vorhanden. Berechnungen mit der Finite-Elemente-Methode haben gezeigt, dass die experimentellen Veränderungen an der Oberfläche nur durch Ausscheidungen zylinderförmiger Geometrie erklärt werden können, die vertikal durch die komplette Schicht reichen (vgl. Abb. 4.23, S. 73). Die Berechnungen haben zusätzlich gezeigt, dass bei den größten Typ T1-Ausscheidungen genug elastische Energie gespeichert ist damit sich ein zylinderförmiger Versetzungsring um eine Ausscheidung bilden kann (vgl. Abb. 5.2, S. 86). Wenn die elastische Energie ausreicht, werden Versetzungen gebildet und aus Typ T1-Ausscheidungen werden inkohärente Typ T2-Ausscheidungen. In sehr dünnen Schichten liegen aufgrund der geringen elastischen Energie nur kohärente Typ T1-Ausscheidungen vor (vgl. $20 \mathrm{~nm}$ dünnen Niobfilm aus Abb. 4.30, S. 81).

Die inkohärenten Typ T2-Ausscheidungen unterscheiden sich in ihrer Oberflächenmorphologie grundlegend von der $\alpha$-Phase. Bei der Umwandlung zu diesem Ausscheidungstyp wird die Oberflächenunebenheit um den Faktor 3.5 erhöht. Die Erhöhung der Rauigkeit ist bedingt durch das Auftreten von Gleitstufen an der Oberfläche. Die Stufen sind bevorzugt entlang der $\langle 1 \overline{1} 1\rangle$ - und $\langle\overline{1} 13\rangle$-Richtung ausgerichtet und ihre Gleitebenen können zu (12)1) und (011) bestimmt werden (vgl. Abschnitt 4.2.6, S. 64). Die Typ T2-Ausscheidungen wachsen bevorzugt in länglicher Form. Die kurze Achse hat dabei eine Ausdehnung von 100-200 nm, die lange Achse reicht über mehrere $\mu \mathrm{m}$ (vgl. Abb. 4.6, S. 53). Diese langgestreckte Form orientiert sich entlang der elastisch weichen $\langle 111\rangle$-Richtungen des Niobgitters (vgl. Abb. 4.15, S. 63). Vertikal bilden sich die Ausscheidungen durch die komplette Schicht. Der an der Oberfläche gemessene Unterschied in der Dehnung zwischen $\alpha$-Phase und den Ausscheidungen vom Typ T2 ist abhängig von der ursprünglichen Schichtdicke $h_{0}$. Er beträgt $\Delta z=0.068 \cdot h_{0}$, wie dies nach Gl. (2.10) (s. S. 8) bei einer Mischungslückenbreite von $\Delta c=0.5$ bei der Berechnung mit der linearen Elastizitätstheorie für dünne Schichten erwartet wird.

Im Randbereich zwischen $\alpha$-Phase und Typ T2-Hydridausscheidungen werden Veränderungen beobachtet. Durch die große mechanische Spannung, die bei der Hydridbildung entsteht, werden Versetzungen in der Hydridphase gebildet, die als Gleitstufen an der Oberfläche sichtbar sind. Diese Stufen reichen bis zu $120 \mathrm{~nm}$ in das Gebiet der $\alpha$-Phase. Es treten Stufen entlang der $\langle 1 \overline{1} 1\rangle$ - und $\langle\overline{1} 13\rangle$-Richtung auf, wobei die Häufigkeit der beiden unterschiedlichen Richtungen von der Orientierung der Grenze zwischen $\alpha$-Phase und Hydrid abhängt. Die Stufen liegen bevorzugt senkrecht zur Grenze, weil dadurch ein maximaler Spannungsabbau stattfindet (vgl. Abschnitt 5.2.3, S. 90).

Es hat sich ergeben, dass bei dünnen Schichten mit einer offenen Oberflächenstruktur neben vertikalen auch laterale Dehnungen zeigen. Bei einer $20 \mathrm{~nm}$ dünnen Schicht, die an der Oberfläche mäanderförmige Strukturen mit Höhenhüben von $\pm 6 \mathrm{~nm}$ aufweist, wurde eine laterale Ausdehnung von 3(2) nm zu beiden Rändern gemessen (vgl. Abb. 4.31, S. 82). Diese laterale Veränderung der Breite entspricht der für ein Modell mit der Finite-Elemente-Methode berechneten Änderung. Bei dieser 
Berechnung wurde die mäanderförmige Struktur durch einen Draht angenähert, der starr mit einem Substrat verbunden ist (vgl. Abb. 3.25(b), S. 44).

Die Kinetik bei der Hydridbildung lässt sich durch eine Johnson-Mehl-Avrami Kinetik anpassen. Die ermittelten Exponenten liegen im Bereich von $n=1.3$ bis 1.5 (vgl. Abschnitt 4.3, S. 69) und entsprechen daher einem diffusionskontrollierten Wachstum, bei dem die Nukleationsrate abnimmt [Chr65, S. 489]. Die abnehmende Nukleationsrate wurde bei den STM-Messungen direkt an der Oberfläche beobachtet. Hierzu ist die Anzahl der Ausscheidungen als Funktion der Zeit untersucht worden (vgl. Abb. 4.22(a), S. 72). Der Gleichgewichtsendwert des umgewandelten Hydridanteils war bei den eingestellten Drücken von $7 \cdot 10^{-7}$ mbar bzw. $9 \cdot 10^{-7}$ mbar kleiner als eins. Somit gibt es im Gegensatz zu massiven Proben in dünnen Schichten keinen Plateaudruck bei dem die gesamte Probe eine Phasenumwandlung durchführt. Zur vollständigen Beladung muss das äußere chemische Potential erhöht werden (vgl. Abb. 5.9, S. 96). Diese Veränderung lässt sich durch die während der Transformation entstehenden mechanischen Spannungen erklären.

Insgesamt hat sich gezeigt, dass die oberflächensensitive Rastertunnelmikroskopie sehr weitreichende Informationen zur Entmischung in dünnen Schichten liefern kann, sofern zwischen Ausscheidung und Matrix ein deutlicher Unterschied zwischen den Gitterkonstanten besteht. 


\section{Literaturverzeichnis}

[AM76] Neil W. Ashcroft und N. David Mermin. Solid State Physics. Holt, Rinehart and Winston, New York Chicago, 1976.

$\left[\mathrm{ASS}^{+}\right.$97] A. Abromeit, R. Siebrecht, G. Song, H. Zabel, F. Klose, D. Nagengast und $\mathrm{A}$. Weidingerf. Thickness dependence of the hydrogen solubility in epitaxial $\mathrm{Nb}(110)$ films grown on $\mathrm{Al}_{2} \mathrm{O}_{3}(11 \overline{2} 0)$ substrates. J. Alloys Comp., 253-254:58-61, 1997.

[AV78a] G. Alefeld und J. Völkl, Hrsg. Hydrogen in Metals I, Bd. 28 der Topics in Applied Physics. Springer-Verlag, Berlin Heidelberg New York, 1978.

[AV78b] G. Alefeld und J. Völkl, Hrsg. Hydrogen in Metals II, Bd. 29 der Topics in Applied Physics. Springer-Verlag, Berlin Heidelberg New York, 1978.

[BH53] G. Brauer und R. Hermann. Die Hydride und Deuteride von Niob und Tantal. Z. Anorg. Allg. Chemie, 274(1-3):11-23, 1953.

[BRGH82] Gerd Binnig, Heinrich Rohrer, C. Gerber und E. Heibel. Tunneling through a controllable vacuum gab. Appl. Phys. Let, 40(2):178180, 1982.

[BvHL $\left.{ }^{+} 98\right] \quad$ M. Bicker, U. von Hülsen, U. Laudahn, A. Pundt und U. Geyer. Optical deflaction setup for stress measurements in thin films. Rev. Sci. Intrum., 69(2):460-462, 1998.

[Chr65] J. W. Christian. The Theory of Transformations in Metals and Alloys. Perganom Press, 1965.

$\left[\mathrm{CMP}^{+} 06\right] \quad J$. Cížek, O. Melikhova, I. Procházka, G. Brauer, W. Anwand, A. Mücklich, R. Kirchheim und A. Pundt. Defects in nanocrystalline Nb-films: Effect of sputtering temperature. Applied Surface Science, 252(9):3245-3251, 2006.

[COM] COMSOL Multiphysics Version 3.2. http://www.comsol.com. mit „Structural Mechanics Module“. 
[CPB $\left.{ }^{+} 06 \mathrm{a}\right]$ J. Cížek, I. Procházka, G. Brauer, W. Anwand, A. Mücklich, R. Kirchheim, A. Pundt, C. Bähtz und M. Knapp. Defect studies of hydrogen-loaded thin Nb-films. Applied Surface Science, 252(9):3237-3244, 2006.

$\left[\mathrm{CPB}^{+} 06 \mathrm{~b}\right]$ J. Cížek, I. Procházka, G. Brauer, W. Anwand, A. Mücklich, R. Kirchheim, A. Pundt, C. Bähtz und M. Knapp. Study of Hydrogen-Defect Interaction in Thin Nb Film on Si Substrate Using Positron Annihilation. Materials Structure, 13(2):82-89, 2006.

[Cra75] J. Crank. The Mathematics of Diffusion, Kapitel 4. Clarendon Press, Oxford, 2. Auflage, 1975.

[CRIT05] T. Car, N. Radicacute, J. Ivkov und A. Tonejc. Resistivity models of the phase transformation of amorphous $\mathrm{Al}_{78} \mathrm{~W}_{22}$ thin films under isothermal and isochronal conditions. Applied Physics A, 80(5):10871092, 2005.

[Dor02] Martin Dornheim. Spannungen, Dehnungen und Lage der Phasengrenzen in dünnen $\mathrm{Nb}$ - und Y-Schichten bei Wasserstoffbe- und -entladung. Dissertation, Institut für Materialphysik, Universität Göttingen, 2002.

$\left[\mathrm{DPK}^{+} 03\right] \quad$ M. Dornheim, A. Pundt, R. Kirchheim, S. J. v. d. Molen, E. S. Kooij, J. Kerssemakers, R. Griessen, H. Harms und U. Geyer. Stress development in thin yttrium films on hard substrates during hydrogen loading. J. Appl. Phys., 93(11):8958-8965, 2003.

[FG76] E. Fromm und E. Gebhardt, Hrsg. Gase und Kohlenstoff in Metallen. Springer-Verlag, Berlin Heidelberg New York, 1976.

[Gra66] Thomas Graham. On the Absorption and Dialytic Separation of Gases by Colloid Septa. Phil. Trans. Roy. Soc, 156:399-439, 1866.

[GW92] H.-J. Güntherodt und R. Wiesendanger. Scanning Tunneling Microscopy I, Bd. 20 der Springer Series in Surface Science. Springer Verlag Berlin, 1992.

[Haa96] Peter Haasen. Physical Metallurgy. Cambridge University Press, Cambridge, 3. Auflage, 1996.

[HF76] G. Hörz und E. Fromm. Niob, S. 460-494. In Fromm und Gebhardt [FG76], 1976.

[HM84] K. H. Hellwege und O. Madelung. Zahlenwerte und Funktionen aus Naturwissenschaften und Technik, Bd. 18 (III) der Landolt Börnstein. Springer-Verlag, Berlin, 1984. 
[JDD72] L. Johnson, M. J. Dresser und E. E. Donaldson. Adsorption and Absorption of Hydrogen by Niobium. J. Vac. Sci. Technol., 9(2):857860, 1972.

[Kes00] Philipp Kesten. Hochauflösende Untersuchung der lokalen Wasserstoffverteilung in metallischen Multischichten mit Hilfe der tomographischen Atomsonde und der Sekundärionenmassenspektrometrie. Dissertation, Institut für Materialphysik, Universität Göttingen, 2000.

[Kir] Reiner Kirchheim. persönliche Mitteilungen.

$\left[\mathrm{KKM}^{+} 02\right]$ B. Karunagaran, R. T. Rajendra Kumar, D. Mangalaraj, Sa. K. Narayandass und G. Mohan Rao. Influence of thermal annealing on the composition and structual parameters of DC magnetron sputtered titanium dioxide thin films. Cryst. Res. Technol., 37(12):1285-1292, 2002.

[Kro60] F. Kroupa. Circular edge dislocation loop. Czech. J. Phys. B, 10:284293, 1960.

[KS69] A. G. Khachaturyan und G. A. Shatalov. Elastic-interaction potential of defects in a crystal. Sov. Phys. Solid St., 11(1):118-123, 1969 .

$\left[\mathrm{KvdMK}^{+} 00\right]$ J. W. J. Kerssemakers, S. J. van der Molen, N. J. Koeman, R. Günter und R. Griessen. Pixel switching on epitaxial $\mathrm{Pd} / \mathrm{YH}_{x} / \mathrm{CaF}_{2}$ switchable mirrors. Nature, 406:489-491, 2000.

[Lau98] Uwe Laudahn. Spannungen und Dehnung von mit Wasserstoff beladenen Nb-Einfach- und Pd-Nb-Vielfachschichten. Dissertation, Institut für Materialphysik, Universität Göttingen, 1998.

$\left[\mathrm{LFK}^{+} 99\right]$ U. Laudahn, S. Fähler, H. U. Krebs, A. Pundt, M. Bicker, U. V. Hülsen, U. Geyer und R. Kirchheim. Determination of elastic constants in thin films using hydrogen loading. Appl. Phys. Lett., 74(5):647-649, 1999.

[LPB $\left.{ }^{+} 99\right]$ U. Laudahn, A. Pundt, M. Bicker, U. von Hülsen, U. Geyer, T. Wagner und R. Kirchheim. Hydrogen-induced stress in Nb single layers. J. Alloys and Compounds, 293-295:490-494, 1999.

[MA94] Z. Ma und L. H. Allen. Kinetic mechanisms of the C49-toC54 polymorphic transformation in titanium disilicide thin films: A microstructure-scaled nucleation-mode transition. Phys. Rev. B, 49(19):13501-13511, May 1994. 
[MBW97] C. Michaelsen, K. Barmak und T. P. Weihs. Investigating the thermodynamics and kinetics of thin film reactions by differential scanning calorimetry. J. Phys. D, 30:3167-3186, 1997.

[MK87] T. Mütschele und R. Kirchheim. Segregation and diffusion of hydrogen in grain boundaries of palladium. Scripta Metallurgica, 21:135$140,1987$.

[Nik06] Eugen Nikitin. persönliche Mitteilungen, 2006.

[NKP03] Kai Nörthemann, Reiner Kirchheim und Astrid Pundt. Surface modification of Nb-films during hydrogen loading. J. Alloys and Compounds, 356-357:541-544, 2003.

$\left[\mathrm{NKvG}^{+} 99\right]$ D. G. Nagengast, J. W. J. Kerssemakers, A. T. M. van Gogh, B. Dam und R. Griessen. Epitaxial switchable yttrium-hydride mirrors. Appl. Phys. Lett., 75(12):1724-1726, 1999.

[ODD $\left.{ }^{+} 98\right] \quad$ V. Oderno, C. Dupour, K. Dumesnil, A. Mougan, Ph. Mangin und G. Marchal. Hexagonal surface structure duren the first stages of niobium growth on sapphire (11̄̄0). Phil. Mag. Lett., 78(5):419-426, 1998.

[Ohr02] Milton Ohring. Materials Science of Thin Films. Academic Press, San Diego London, 2. Auflage, 2002.

[Omi98] Omicron Vakuumphysik, Taunusstein. Scala Electronics Technical Reference Manual, 1998.

[Ori78] R. A. Oriani. Hydrogen embrittlement of steels. Ann. Rev. Mater. Sci., 8:327-358, 1978.

[Pas63] D. W. Pashley. The Growth and Structure of Thin Films. In Thin Films, Kapitel 3, S. 59-98. American Society for Metals, 1963.

[Pei78] H. Peisl. Lattice Strains due to Hydrogen in Metals. In Alefeld und Völkl [AV78a], Kapitel 3.

$\left[\mathrm{PGB}^{+} 00\right] \quad$ A. Pundt, M. Getzlaff, M. Bode, R. Kirchheim und R. Wiesendanger. H-induced plastic deformation of Gd thin films studied by STM. Phys. Rev. B, 61:9964, 2000.

[PK06] A. Pundt und R. Kirchheim. Hydrogen in metals: Microstructural aspects. Annu. Rev. Mater. Res., 36:555-608, 2006. 
[PNS06] Astrid Pundt, Kai Nörthemann und Sönke Schmidt. Hydrogenrelated Surface Modifications of $20 \mathrm{~nm}$ thin Straight-sided Niobium Nano-Wires and Niobium Meander-Films. submited to J. Alloys and Compounds, 2006.

[PT69] J. A. Pryde und C. G. Titcomb. Solution of Hydrogen in Niobiom. Trans. Faraday Soc., 65(562):2758-2765, 1969.

[QVLW83] Zh. Qi, J. Völkl, R. Lässer und H. Wenzl. Tritium diffusion in V, Nb and Ta. J. Phys. F, 13:2053-2062, 1983.

[Rob00] S. Roberts. Microplasticity. http://users.ox.ac.uk/ roberts/sgrgroup/ lectures/microplast/microplast_main.htm, 2000.

[RPBS96] A. E. Romanov, W. Pompe, G. E. Beltz und J. S. Speck. An aproach to threading dislocation "reaction kinetics". Appl. Phys. Lett., 69(22):3342-3344, 1996.

[San99] D. Sander. The correlation between mechanical stress and magnetic anisotropy in ultrathin films. Reports on Progress in Physics, 62(5):809858, May 1999.

[Sch74] Gustav E. R. Schulze. Metallphysik. Springer-Verlag, Wien New York, 1974.

[SGAZ96] G. Song, M. Geitz, A. Abromeit und H. Zabel. Solubility isotherms of hydrogen in epitaxial $\mathrm{Nb}(110)$ films. Phys. Rev. B, 54(19):14093-14101, 1996.

[Sie29] A. Sievert. Die Aufnahme von Gasen durch Metalle. Z. Metallkunde, 21:37-41, 1929.

[SK93] Joseph A. Stroscio und William J. Kaiser, Hrsg. SCANNING TUNNELING MICROSCOPY, Bd. 27 der METHODS OF EXPERIMANTAL PHYSICS. Academic Press Inc, 1993.

[SR04] Norbert Schwarzer und Frank Richter. On the determination of film stress from substrate bending: STONEY's formula and its limits. http://archiv.tu-chemnitz.de/pub/2006/0011, 2004.

[SRTBZ97] G. Song, A. Remhof, K. Theis-Bröhl und H. Zabel. Extraordinary adhesion of niobium on sapphire substrates. Phys. Rev. Lett., 79(25):5062-5065, Dec 1997.

[Sto09] G. Gerald Stoney. The Tension of Metallic Films deposited by Electrolysis. Proc. Roy. Soc. A, 82(553):172-175, May 1909. 
[STY89] T. Suzuki, S. Takeuchi und H. Yoshinaga. Dislocation Dynamics and Plasticity, Bd. 12 der Springer Series in Material Science. SpringerVerlag, 1989.

[SW78] T. Schober und H. Wenzl. The System NbH(D), TaH(D), VH(D). In Alefeld und Völkl [AV78b], Kapitel 2.

[SZ01] Louis Schlapbach und Andreas Züttel. Hydrogen-storage materials for mobile applications. Nature, 414:353-358, 2001.

[Tei] H. Teichler. persönliche Mitteilungen.

[Voi28] Woldemar Voigt. Lehrbuch der Kristallphysik. Teubener, Leipzig und Berlin, 2. Auflage, 1928.

[WG92] R. Wiesendanger und H.-J. Güntherodt. Scanning Tunneling Microscopy II, Bd. 28 der Springer Series in Surface Science. Springer Verlag Berlin, 1992.

[Wie94] R. Wiesendanger. Scanning Probe Microscopy and Spectroscopy. Cambridge University Press, 1994.

[WMTB01] A. R. Wildes, J. Meyer und K. Theis-Bröhl. The growth and structure of epitaxial niobium an sapphire. Thin Solid Films, 401(1$2): 7-34,2001$.

[WR70] R. H. Wiswall und J. J. Reilly. The Higher Hydrides of Vanadium and Niobium. Inorganic Chemistry, 9(7):1678-1682, 1970.

[WSx] WSxM ${ }^{\circledR}$. http://www.nanotec.es.

[WW92] Johannes Weertman und Julia R. Weertman. Elementary Dislocation Theory. Oxford University Press, New York Oxford, 1992.

[YDF78] H. Yamauchi und D De Fontaine. Elastic interaction of defect clusters whith arbitrary strain fields in an anisotropic continuum. Acta. Met., 27:763-776, 1978.

[YSF $\left.{ }^{+} 96\right] \quad$ Q. M. Yang, G. Schmitz, S. Fähler, H. U. Krebs und R. Kirchheim. Hydrogen in Pd/Nb multilayers. Phys. Rev. B, 54(13), 1996.

[YZSK95] K. Yoshii, H. Zamamoto, K. Saiki und A. Koma. Superconductivity and electrical properties in single-crystaline ultrathin $\mathrm{Nb}$ grown by molucar-beam epitaxy. Phys. Rev. B, 52(18):13570-13575, 1995.

[Zep] Reiner Zepernick. persönliche Mitteilung. Bestec GmBH, Berlin. 


\section{Worte des Dankes}

Nun bin ich an der Stelle gekommen, mich bei denen zu bedanken, die mir bei der Anfertigung dieser Arbeit zur Seite standen.

Frau PD. Dr. Astrid Pundt danke ich für die intensive Betreuung, viele Anregungen und stetige Diskussionen.

Bedanken möchte ich mich bei Herrn Prof. Dr. Reiner Kirchheim, der Anfertigung dieser Doktorarbeit im Institut für Materialphysik ermöglichte.

Bei Kevin Brinkmann, Joachim Bankmann und meiner Schwester Iris möchte ich mich für das Korrekturlesen dieser Arbeit bedanken.

Den anderen Mitgliedern (auch ehemaligen) der Wasserstoff-Gruppe danke ich für eine gute Arbeitsatmosphäre und viele Gruppensitzungen mit interessanten Diskussionen und Fragen, die diese Arbeit vorangebracht haben. Ein besonderer Dank gilt dabei Philipp Kesten, Martin Dornheim, Diana Marcano, Olof Dankert und Sönke Schmidt.

Der Dank beim technischen Personal des Instituts darf nicht ausbleiben, denn ihre Hilfe hat einige Messungen dieser Arbeit erst möglich gemacht. So danke ich den Laboranten Matthias Hahn, Karin Ahlborn und Mike Kanbach, das sie bei kleinen und großen Problemen stets schnell geholfen haben. Bei Thorsten Nägel, Michael Hey und Denny Wagner aus der elektronischen Werkstatt bedanke ich mich für die kleinen und großen Bauteile und Geräte, die diese Arbeit vereinfacht haben. Aus der feinmechanischen Werkstatt bedanke ich mich besonders bei Frank Köhler und Volker Radisch.

Ebenfalls zu danken habe ich auch allen anderen Mitarbeitern des Instituts für Materialphysik, die für ein angenehmes Arbeitsklima sorgten und durch Unterstützung und Anregungen zum Gelingen dieser Arbeit beitrugen. Die Kaffeerunde sorgte für schöne fachfremde und physikalische Diskussionen.

Dank gebührt auch denjenigen Programmierern die ihre Software frei zur Verfügung gestellt haben und mir somit Programmierarbeit erspart und vereinfacht haben.

Bei meinen Eltern möchte ich mich für die vorbehaltlose Unterstützung meines ganzen Studiums bedanken.

Mein größter Dank geht an meine Freundin Diana, die mich stets unterstützt hat. 



\section{Lebenslauf}

\section{Persönliche Daten}

$\begin{array}{ll}\text { Name: } & \text { Kai Nörthemann } \\ \text { Geburtsdatum: } & 24.07 .1973 \\ \text { Geburtsort: } & \text { Duderstadt } \\ \text { Familienstand: } & \text { ledig } \\ \text { Nationalität: } & \text { deutsch }\end{array}$

\section{Ausbildung}

\begin{tabular}{|c|c|c|}
\hline chule & $\begin{array}{l}1980-1984 \\
1984-1986 \\
1986-1990 \\
1990-1993 \\
5 / 93\end{array}$ & $\begin{array}{l}\text { Grundschule Gerblingerode } \\
\text { St. Ursula-Schule Duderstadt } \\
\text { Realschule Duderstadt } \\
\text { Eichsfeld-Gymnasium Duderstadt } \\
\text { Allgemeine Hochschulreife }\end{array}$ \\
\hline Studium & $\begin{array}{l}10 / 93-10 / 98 \\
3 / 97-9 / 98\end{array}$ & $\begin{array}{l}\text { Physikstudium an der Georg-August- } \\
\text { Universität zu Göttingen } \\
\text { Diplomarbeit in Physik an der } \\
\text { Forschungseinrichtung Röntgenphysik } \\
\text { unter der Anleitung von Prof. Dr. G. Schmahl } \\
\text { zum Thema: } \\
\quad \text { Untersuchungen zu Kondensoren für das } \\
\text { Transmissionsröntgenmikroskop am BESSY II } \\
\text { Diplom in Physik; Note: gut }\end{array}$ \\
\hline & seit 3/99 & $\begin{array}{l}\text { Promotionsstudiengang am Institut für } \\
\text { Materialphysik in der Arbeitsgruppe von } \\
\text { PD. Dr. A. Pundt }\end{array}$ \\
\hline
\end{tabular}

\section{Berufstätigkeit}

seit 3/99 Wissenschaftlicher Angestellter an der

Georg-August-Universität zu Göttingen 